SSC-SR-1001

\title{
Report of the Task Force on \\ SSC Magnet System Test Site
}

\section{DISCLAIMER}

This report was prepared as an account of work sponsored by an agency of the United States Government. Neither the United States Government nor any agency thereof, nor any of their employees, makes any warranty, express or implied, or assumes any legal liability or responsibility for the accuracy, completeness, or usefulness of any information, apparatus, product, or process disclosed, or represents that its use would not infringe privately owned rights. Reference herein to any specific commercial product, process, or service by trade name, trademark, manufacturer, or otherwise does not necessarily constitute or imply its endorsement, recommendation, or favoring by the United States Government or any agency thereof. The views and opinions of authors expressed herein do not necessarily state or reflect those of the United States Government or any agency thereof. 


\section{LEGAL NOTICE}

This book was prepared as an account of work sponsored by an agency of the United States Government. Neither the United States Government nor any agency thereof, nor any of their employees, makes any warranty, express or implied, or assumes any legal liability or responsibility for the accuracy, completeness, or usefulness of any information, apparatus, product, or process disclosed, or represents that its use would not infringe privately owned rights. Reference herein to any specific commercial product, process, or service by trade name, trademark, manufacturer, or otherwise, does not necessarily constitute or imply its endorsement, recommendation, or favoring by the United States Government or any agency thereof. The views and opinions of authors expressed herein do not necessarily state or reflect those of the United States Government or any agency thereof. 


\section{DISCLAIMER}

Portions of this document may be illegible in electronic image products. Images are produced from the best available original document. 
SSC-SR-1001

\section{Report \\ of the \\ Task Force \\ on \\ SSC Magnet System Test Site}

October 1984

DISTRIBUTION OF THIS DOCUMENT IS UNLIMITEP A NASSTR 
This report by the Task Force on SSC Magnet Systems Test Site was prepared in response to the charge by Maury Tigner, Director of SSC Phase I, asking the Task Force to make a critical evaluation of potential sites for a major SSC magnet System Test Facility (STF). The charge suggested that, because of existing facilities and experienced staffs, the Brookhaven National Laboratory (BNL) and the Fermi National Accelerator Laboratory (FNAL or Fermilab) are the two best candidate sites. Our subsequent deliberations did not lead to any dissensions from this opinion.

The Task Force held four meetings. In the first meeting, descriptions of the test facility and test program and "specifications" of the facility were developed. In the second and third meetings, the Task Force listened to presentations by the staffs of the two candidate laboratories and visited the existing facilities at these locations. The fourth meeting was devoted to review of the up-dated responses of FNAL and BNL to the Test Facility specifications, discussion of our findings and conclusions, and editing of the entire report.

The basic conclusions and recommendations of the Task Force are as follows:

- Because of overall SSC schedule considerations, the planning of the System Test Facility must be flexible enough to accommodate any one of the three magnet types.

- The complete System Test Facility should provide two test vehicles: 1) a "Short String Facility" on which "wear-out" tests can be run; and 2) a "Long String Facility" where extensive testing and development activities involving the principal modular lengths of magnets and related systems can be conducted.

- The Long String Facility configuration should be straight (or slightly curved if necessary to conform to an existing housing).

- The length of the Short String Facility should be one half-cell. The length of the Long String Facility should be $\sim 1 \%$ of the circumference of the Main Ring. For early planning purposes and for the estimates made in this report, a length of $1.2 \mathrm{~km}$ will be used. The Long String Facility should provide for the testing of magnet systems that include both beam apertures.

- Initial tests at the STF should start a few months after magnet type selection. A full complement of magnets should be in place about a year later, permitting long string 
tests. Both short string and long string tests may continue in parallel during the life of the facilities.

- Integrated testing and validation of magnets, cryogenic system, power supplies, quench detection and protection systems, control, vacuum system, correction coils and safety systems, all considered as a total systems complex, should be a principal objective at the System Test Facility.

- Plans should be made to install up-graded versions of the various systems as they become available during the SSC R\&D and design phases. The cost of up-grading these systems is not included in this report.

- Transfer of the test program to the construction site should be started about 1-1/4 years after SSC construction authorization. Operations at the STF should continue until the move is fully accomplished.

- At the construction site, it would be highly beneficial to install the final versions of the test systems, i.e., prototypes of the SSC systems, in a section of the SSC tunnel that is expressly constructed on an advanced schedule for this purpose.

- In its evaluation procedures, the Task Force has given primary attention to the Laboratories' capabilities with respect to the installation, operation and testing of magnets and magnet cryogenic systems. This emphasis is due to their basic importance in the overall SSC Facility and because they must be in place and must function properly to permit optimal development of the principal ancillary systems.

- Both BNL and FNAL managements and staffs have exhibited commendable enthusiasm for this national effort and have obviously given considerable thought and effort to the preparation of the Laboratories' responses.

- Both the BNL response and the FNAL response satisfy the basic requirements of the STF as outlined in this report.

- Manpower requirements at either Laboratory would be approximately the same for magnet types $\mathrm{A}, \mathrm{B}$, and $\mathrm{C}$.

- Total professional effort (in man years) would be about the same at either Laboratory.

- Total technical manpower effort (in man years) would be approximately $50 \%$ higher at BNL than at FNAL.

- The comparisons made by the Task Force indicate that the total costs of siting the STF at BNL and FNAL are roughly equal; the cost difference is probably within the uncertainties stemming from the basic assumptions of this review.

- The cost items presented herein are only a part of those required for the proposed string tests. In particular, the magnets and their cryostats, which are expected to be major cost items, are not included in the estimates.

- The charge to the Task Force does not ask for a specific site recommendation for the STF. The siting decision must ultimately be made by SSC management on the basis of the relative weights given to relevant selection factors and the degree of success in negotiating a satisfactory contractual agreement with the favored Laboratory. 
- The STF will be a major component of the national SSC program, involving scientists from many different institutions. It is expected that the SSC Central Design Group will play a major role in this activity by providing technical, management, and budgetary input and direction. 



\section{Contents}

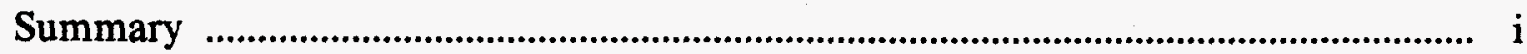

I. Introduction ............................................................................................... 1

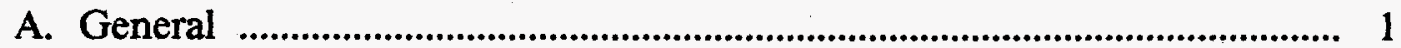

B. System Test Facility Schedule ………............................................................ 2

C. Integrated System Testing ….................................................................... 4

D. Size and Configuration of the Long String Facility ..................................... 5

E. Caveats Regarding Use of Cost Estimates .......................................................... 5

II. Purpose of the System Test Facility .................................................................. 7

III. Test Program ........................................................................................... 9

A. Rationale for the Test Program .............................................................. 9

B. Description of the Test Program ................................................................ 13

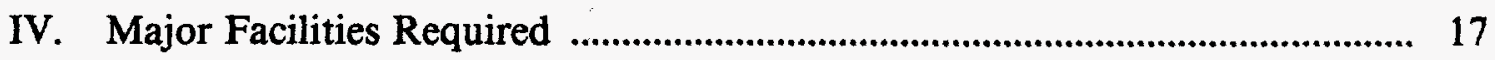

A. Refrigeration System ............................................................................ 17

B. Power Supply System ................................................................................ 19

C. Control, Logging, and Simulation System .............................................. 20

D. Quench Detection and Protection System ................................................ 21

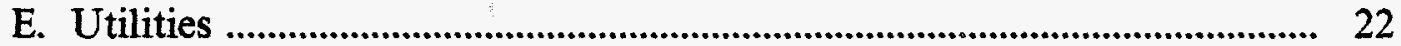

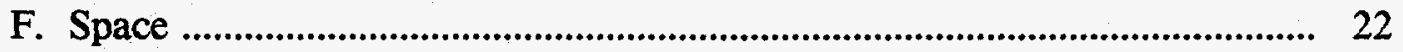

G. Miscellaneous ....................................................................................... 23

V. Alternative Locations of the System Test Facility .............................................. 25

A. Summary of the BNL Response .............................................................. 25

B. Summary of the FNAL Response ........................................................ 26

C. Summary Tables from BNL/FNAL ....................................................... 27

VI. Comparisons and Conclusions ….................................................................... 65

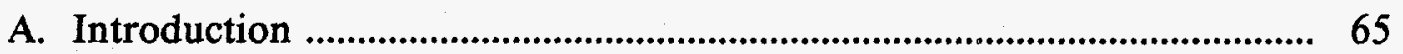

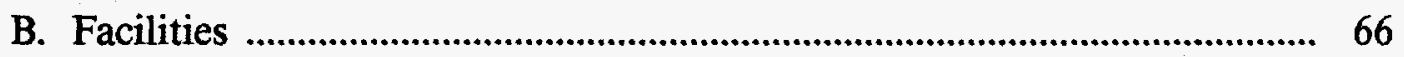

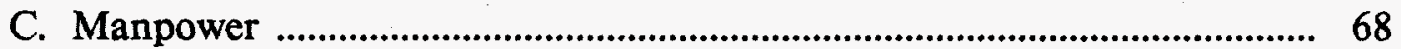

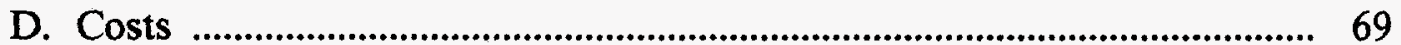

E. General Remarks on STF Site Selection ..................................................... 69

Appendices:

A. Membership of the Task Force ….......................................................... A-1

B. Task Force Charge ......................................................................... B-1

C. Meeting Agenda ....................................................................................... C-1

D. BNL Response to the Test Facility Specifications .................................... D-1

E. FNAL Response to the Test Facility Specifications .................................... E-1 


\section{Introduction}

\section{A. General}

The Task Force on SSC Magnet Systems test Site was appointed by Maury Tigner, Director of the SSC, Phase I in August, 1984. The membership of the Task Force is given in Appendix A and its charge is shown in Appendix B.

In brief, the charge asked the Task Force to make a critical evaluation of potential test sites for a major SSC magnet System Test Facility (STF) with regard to: (1) availability of the needed space, utilities, staff and other requirements on the desired time scale; and (2) the cost of preparing the sites for the tests and for operating the facilities during the test period. The charge further suggests that, by virtue of existing facilities and availability of experienced staff, BNL and FNAL are the two best candidate sites and that it therefore appears appropriate to restrict the considerations of the Task Force to these sites. During the subsequent deliberations of the Task Force, no new facts were revealed that altered the assumptions of the charge in this regard.

The charge does not ask for a specific site recommendation for the STF. Indeed, an agreement on such a recommendation would be difficult to achieve considering the composition of the Task Force, wherein a large fraction of the membership is drawn from the two contending laboratories. Instead, we have attempted to describe the purpose of the facility, outline a productive test program, list the major facilities required, carefully review the laboratories' responses to the facility requirements, and make objective comparisons of the specific features and capabilities offered.

The Task Force held four meetings as follows:

September 13-14 (SLAC)

Descriptions of the test system and test program for each of the three magnet types were discussed and agreed upon. 
Specifications of the major facilities needed to carry out the suggested test programs were compiled and estimates of the manpower required to prepare and operate the facilities were discussed.

September 25 (FNAL) and September 26-27 (BNL)

During these visits the Task Force listened to presentations by the staffs of each Laboratory regarding the adequacy of their present facilities and available staff for the intended SSC magnet system test program, what modifications or additions would have to be made, and the associated costs. We visited the existing facilities and discussed our observations and findings.

On the second day of the BNL visit, the Task Force held further discussions and worked on the draft of its report.

October 11-12 (O'Hare Hilton Hotel)

This two day meeting was devoted to review of the up-dated responses to the System Test Facility specifications from FNAL and BNL, discussion of our findings and conclusions, and to editing of the entire report.

\section{B. System Test Facility Schedule}

To respond to its charge, it was first necessary for the Task Force to establish a schedule for the STF. Key dates obtained from various SSC documents and from M. Tigner were used along with other dates recommended by this Task Force as the basis of the schedule shown in Figure I.1. Some of the implications of this schedule with respect to our deliberations will now be discussed.

The decision regarding location of the STF will be made by SSC management in December 1984, whereas the selection of the magnet type will not be made until about a year later, at the end of 1985. It is desirable to start installation and testing of the selected magnet type at the Long String Facility as soon as possible after the magnet selection is made in order to minimize the SSC development and construction period. This requires that the design and construction of the STF start immediately after the site is selected. The fact that the decision regarding magnet type will not be made until about a year later means that the STF design must be flexible enough to accommodate any of the 3 magnet types. Therefore, the Task Force has found it necessary to define the specifications for the STF on a broad, flexible basis permitting such accommodation.

Installation of the magnets at the STF will start late in calendar year 1985 and continue through 1986 and possibly into the first half of 1987. Magnets will be shipped to and installed in the STF as they become available from the Magnet Pre-production Facility; tests of short sections will then start in the Long String Facility as soon as the overall state of readiness permits. The assumed schedule indicates that a full complement of magnets will be in place by the end of 1986 . Thus, long string tests can get underway in early 1987 . 
Calendar Year

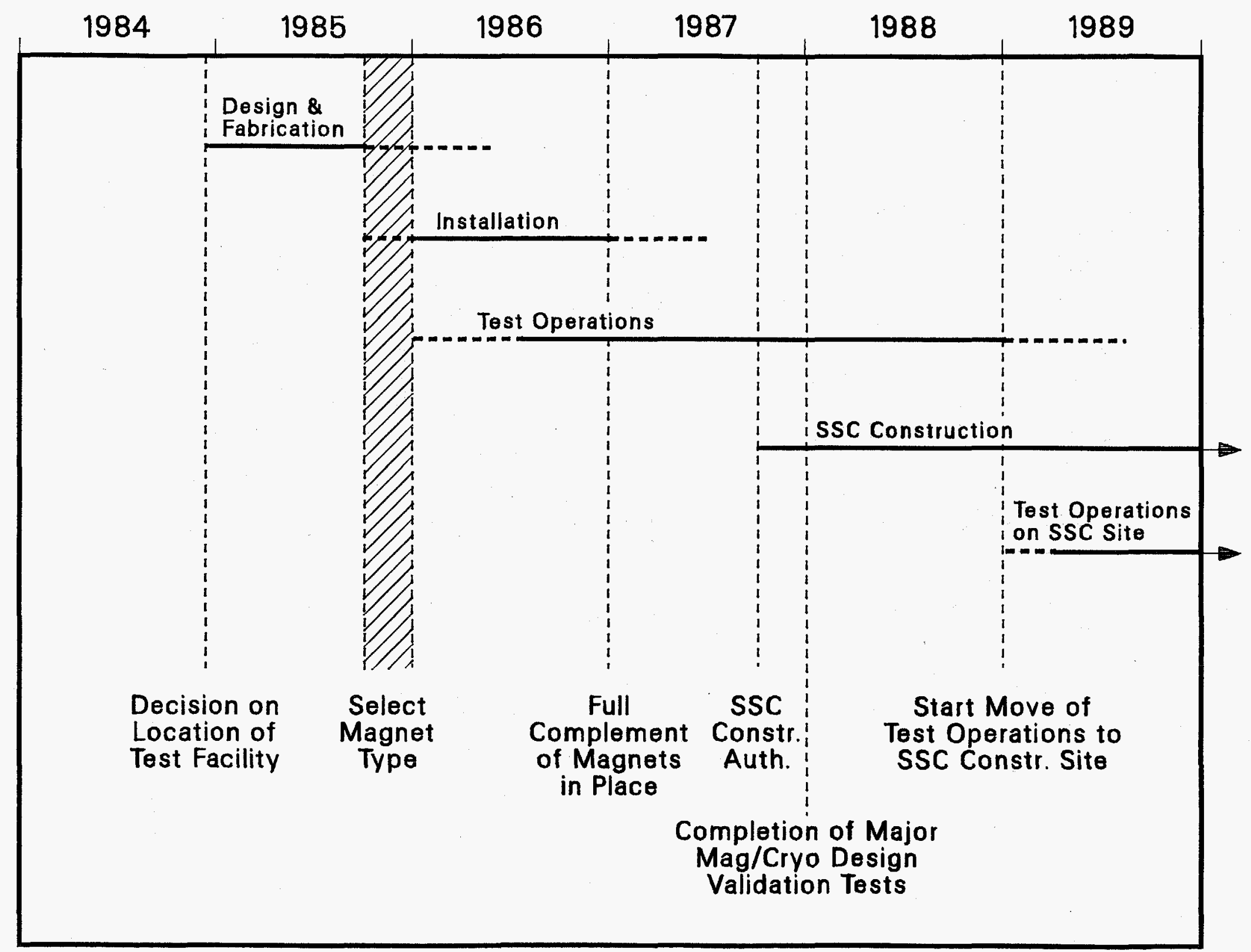

XCG 8410-13379

Fig. I.1 Assumed Schedule SSC Magnet System Test Facility. 
The schedule assumes that U.S. Congress will authorize construction of the SSC in FY 1988 and that construction activities will begin at the SSC site shortly thereafter. Operations at the STF are expected to continue for at least 18 months after construction authorization. By early 1989, the state of construction should permit starting the move of test operations to the SSC site. It would be highly beneficial to install the prototype systems in a section of the SSC tunnel that is expressly constructed on an advanced schedule for this purpose. This would allow early operation of these systems in the actual physical environment that the entire accelerator will experience later. Such operating experience would be extremely valuable in identifying design problems prior to completion of a large fraction of the accelerator. Necessary modifications could then be made on a timely basis resulting in significant cost savings and preventing schedule slippages that might otherwise occur. Similar plans for early operation of a portion of the accelerator were followed at SLAC and FNAL with highly salutary results. If such a plan is adopted for the SSC, the components and systems installed on the early schedule will likely not consist of items moved from the STF but will consist of units of the latest (and hopefully final) designs that are to be fabricated and installed in the main ring of the SSC. In this manner, the ensuing test results will provide the final check and validation of the integrated accelerator systems of the SSC.

\section{Integrated System Testing}

The costs estimated in this report are associated with the preparation of the site and facility for the test program discussed in Section III and for the operation of the facility during the period shown in the assumed schedule (Figure I.1). The early startup date for this facility, which follows the magnet decision date by only a few months, makes it infeasible to use initially the actual SSC designs for auxiliary systems such as cryogenics, power supply, and control systems since these systems will not have been designed. On the other hand, it is clear that the significance and validity of the test program would be increased greatly if some or all of these systems could be incorporated in the Long String Facility as soon as they are available. Moreover, such action would provide the opportunity to measure the performance of these auxiliary systems in a total-system operating environment and to make technical improvements as appropriate over a period of several years.

Even though systems designed specifically for the SSC will not be available at the start of the test program, we feel that it is essential that the test program be planned to evaluate, not just the magnets, but the performance of the integrated SSC magnet system, i. e., the magnets, cryogenic system, power supply, quench detection and protection systems, control system, vacuum system, correction coils and safety systems representing the continually up-graded designs arising from the total development effort.

For the purpose of this study, we estimate the incremental cost of only the initial complement of equipment constituting the STF; we assume that the cost of the follow-on versions of these systems will be supported by the regular engineering and development categories provided in the overall SSC budget plan. The STF cost and manpower plan given in this report will provide the staff necessary to support the installation, operation, and maintenance of these evolving systems but will not support the continuing design effort on these systems or the cost of the various stages of replacement hardware. 


\section{Size and Configuration of the Long String Facility}

The need to obtain test results involving the principal modular lengths of various key SSC systems, as discussed further in Section III, imposes a rough lower limit to the size of the test facility and the number of magnets and other components that it includes. The upper limit is determined by budget and schedule considerations and by the space and facilities available at the candidate sites, viz., FNAL and BNL. Discussion of these factors by the Task Force led to the conclusion that the length of the Long String Facility should be approximately $1 \%$ of the circumference of the main ring associated with each of the 3 magnet types. On this basis, the length of the facility would need to be $0.9,1.1$, or $1.6 \mathrm{~km}$ depending upon which magnet type is selected. Since the length is not a sharply defined quantity we have, to simplify the estimates made in this report, assumed that the facility length will be $1.2 \mathrm{~km}$ for any of the three magnet types. Space of this magnitude is available at both FNAL and BNL.

We considered the advisability of shortening the amount of space required and possibly reducing the total cost by folding the long string back on itself one or more times. While this scheme is technically possible, we advise against it since the test results will depend upon the geometry of the facility to a degree that is very difficult to predict. Confidence in the results would be decreased and therefore the basic objective of the test program, which is to validate the designs of the SSC accelerator systems, would be impaired. Thus, we recommend that the test facility configuration be straight (or slightly curved to conform to an existing housing).

\section{E. Caveats Regarding Use of Cost Estimates}

The cost estimates made in this report are for comparison of the merits of locating the STF at two alternate laboratories, FNAL and BNL. Caution should be exercised in using these estimates for other purposes, in particular for preparing budget documents. As noted in later sections, our estimates do not include G\&A (overhead), escalation or contingency. In addition, certain costs that are essentially identical for both laboratories have been omitted in some instances. Finally, it should be emphasized that there are many other very significant costs associated with establishing and operating a major test facility that are beyond the scope of this report. These include the cost of the magnets and their shipment to the test site and, as mentioned in Section I.C above, the continual up-grading of the systems in consonance with the on-going SSC development program. 


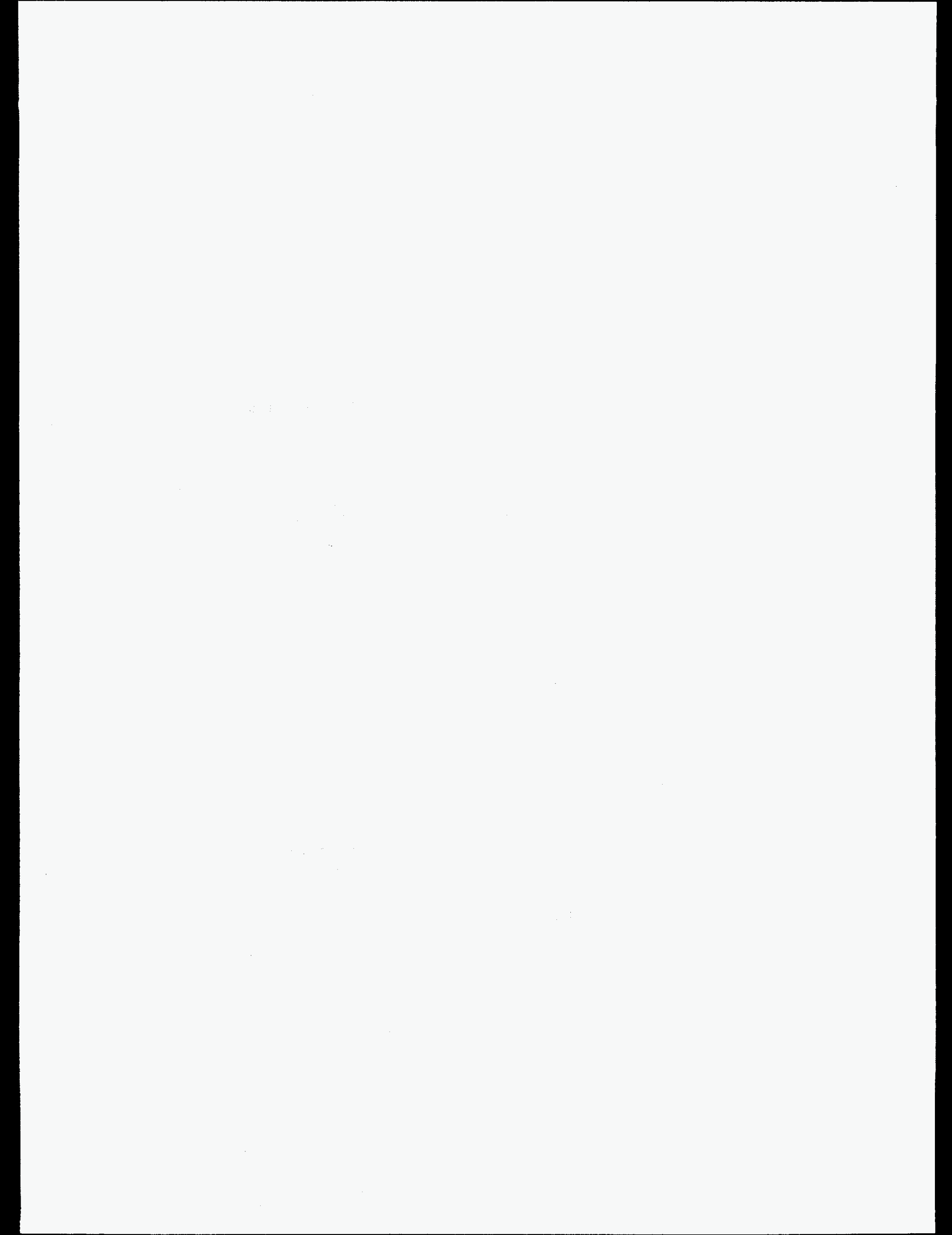




\section{Purpose of the System Test Facility}

The purpose of the STF is to generate and answer questions that arise concerning those aspects of SSC design that apply to a relatively large group of magnets, as opposed to single magnet tests. In addition to being used for system design and test, the STF will also be used to perform life tests on magnets and other accelerator components.

It is assumed in this description that questions of field quality, single magnet quench propagation, and other issues that can be investigated with single magnet tests will be addressed in a different facility, such as Fermilab's MTF, or BNL's Magcool.

It is expected that the STF will be used to develop and verify designs in the following systems:

1. Magnet systems.

2. Magnet cryogenics.

3. Power supplies.

4. Quench detection and protection.

5. Controls.

6. Vacuum.

7. Correction coils.

8. Safety.

Because functional magnets and magnet cryogenic systems are required to develop and evaluate the other systems above, the Laboratories' capabilities with respect to these two items are emphasized in this report.

It is important to note that this facility will not be used to test the refrigerator, but will be used to collect cryogenic data relevant to its design.

In general, the SSC schedule (Fig. I.1) is such that the STF must be available to operate some small number of magnets by January 1986 and progress to a large scale test $(\sim 1 \%$ of the SSC circumference) by early 1987 , with the initial major tests complete by the end of CY 1987. It should be emphasized that the STF will play a critical role in the R\&D effort long after the end of CY 1987. It is reasonable to assume that operations of the STF will 
continue until tests of the final magnet are completed and, hence, the STF may be operated until a good fraction of the ring itself is installed in the real tunnel. Because of the continued need for this test facility, it is unlikely that the STF will be shut down to be moved to the SSC site. The first tests at the SSC site may very well be a section of the SSC installed in the tunnel.

The test program has two major goals. The primary goal is to develop and test the systems listed above, especially the magnet systems, leading to a good overall SSC design. The second goal is to perform life tests. To this end, there are two independent strings of magnets envisioned for the STF. One will eventually grow to be the order of one percent of the total SSC in length, including both apertures; the other will be shorter, consisting of one half-cell of the SSC.

The long string has two phases of operation:

PHASE I: Early, small scale tests, in which the major purpose is to get some small number of magnets operating. The goal of these tests is to see if there are any obvious problems with systems, such as installation, heat leak, quench propagation and protection, etc.

PHASE II: Increasingly larger scale tests, in which each subsystem progresses toward its final design state, and in which the STF assumes its role as a test bed for many subsystems. Phase II will be used for life tests, power supply design, controls system optimization, etc. It is expected and desired that as subsystems for the SSC are designed, they will be tested and developed in the long string during the later parts of the Phase II operation. It is clear that this use for the long string will require its operation to extend well into the construction phase of the SSC project.

Because of the need to make a smooth transition between Phases I and II, it is important that subsystems utilized for Phase I be conceived with the idea of evolving into the final design. In other words, although it is realized that the changes in the details of many subsystems will be profound, the systems should be thought of as being subsets of an integrated whole, not as devices or systems used to perform a temporary and isolated task.

The purpose of the short string is primarily to do thermal and electrical life tests that require a large number of cycles. It is essential that these two strings operate totally independently in terms of refrigeration and power supplies. It is desirable but not necessary that they be at the same site. 


\section{A. Rationale for the Test Program}

In order to specify a test program for SSC magnet strings, it is necessary to develop a rationale for what the tests must accomplish. We address here the particularly troublesome problem of "magnet lifetime". For this discussion we make three assumptions about the SSC.

1. Magnetic Cycles: The lifetime of the machine is 20 years, and it will operate twothirds of the time. If there were two fills per day, then during the lifetime of the machine there will be $2 \times(2 / 3) \times 365 \times 20$, or about $10^{4}$ magnetic cycles.

2. Quenches: We assume one quench per day somewhere in the ring yielding $1 \times(2 / 3)$ $\times 365 \times 20$ or about 5,000 quenches over the life of the machine. If the string is 1 percent of the ring then $\mathbf{5 0}$ quenches in the string correspond to the ring average.

3. Thermal Cycles: We assume one thermal cycle to ambient temperature per month somewhere in the ring, giving $1 \times 12 \times 20$ or about 240 cycles over the machine lifetime. Again, if the string is 1 percent of the ring, it can be expected to undergo about 3 cycles.

Now we consider how these numbers relate to magnet testing. First, it must be recognized that the expected lifetime of the component parts of a magnet can be obtained by well known techniques. For instance, the problem of collar fatigue failure was studied in the Tevatron by constructing a mechanical device that would cycle a small section of the collars through many cycles. If the number of cycles to failure versus force is plotted, one obtains a curve of the type shown in Fig. III.1. 


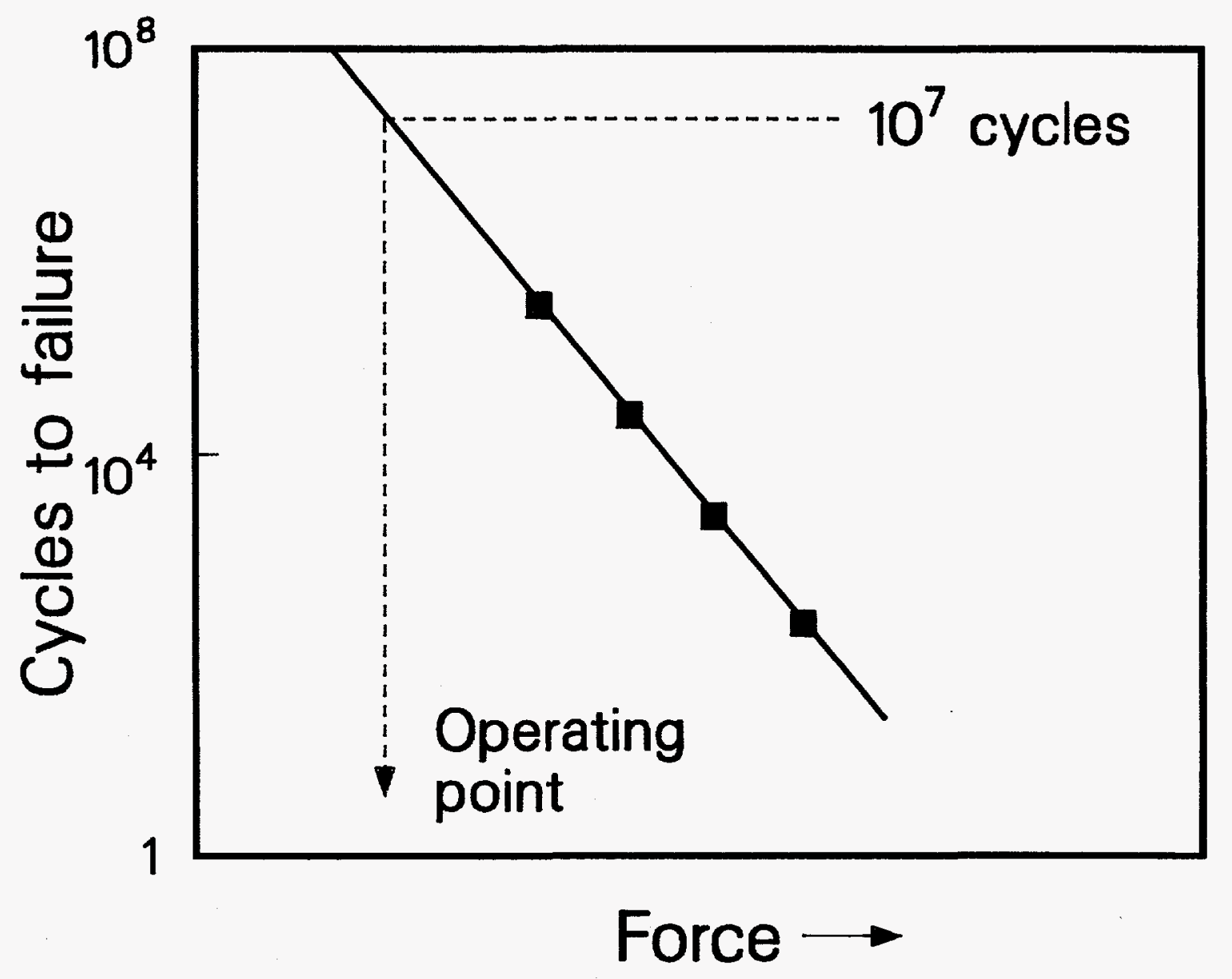

XCG 8410-13378

Fig. III.1. Typical Failure Curve for Cyclically Stressed Components.

Thus, although $10^{7}$ cycles is very hard to obtain, the curve allows an empirical prediction of the lifetime by studying collar failure at a stress higher than that calculated for normal operating conditions. Note that the extrapolation is "safe" in that the real lifetime would be expected to be larger than predicted by this technique (i.e., at zero force the lifetime should approach infinity). Similarly, the coil matrix (wire + insulation) can be cyclically stressed, and its lifetime estimated. These techniques follow standard engineering practice and are used in qualifying airplanes and other structures that must have a very low risk of failure in order to be acceptable to the user.

We, therefore, assume that the magnet during its development has had extensive and systematic studies performed on its components. It should be possible to design these component parts such that the body of the magnet can be made to withstand several orders of magnitude more magnetic cycles than anticipated in the SSC lifetime $\left(10^{4}\right)$.

We assume that the failure curve of Fig. III.2 will qualitatively apply to the SSC magnet system. 


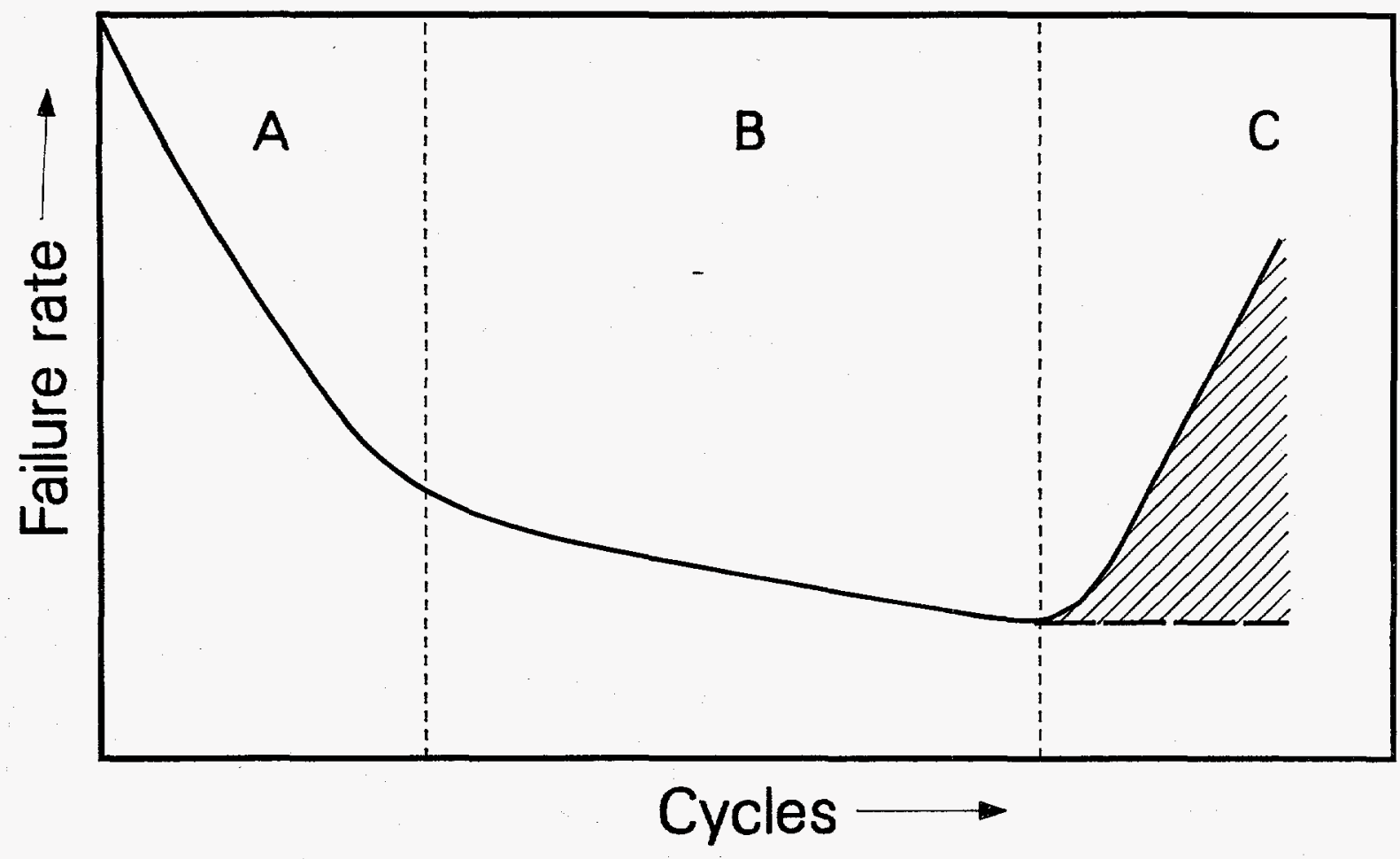

XCG 8410-13377

Fig. III.2 Generic Failure Curve. The Regions are: A - Infant Mortality, B Midlife (uncontrolled causes), and $\mathrm{C}$ - Wearout.

The three regions of the curve refer to: A. infant mortality, B. uncontrolled causes, and C. "wear out." Region A is controlled by careful manufacturing techniques. Region $\mathrm{C}$ is caused by such things as collar fatigue and is studied in the body of single magnets by techniques indicated above.

Region B of the curve is hardest to address by string tests. To set the scale, we note that the Tevatron now has about $10^{5}$ cycles on 1,000 magnets or $10^{8}$ magnets $\times$ cycles. At this point, all magnet failures have been identified as either $\mathrm{A}$ or $\mathrm{C}$ region failures and consequently we have only a limit on the B region for Tevatron magnets. Further operation will push this product higher by a factor of 10 in the next few years. For the SSC we expect approximately $10^{8}$ magnets $\times$ cycles, a number similar to what has already been obtained at the Tevatron. 
In addition to the lifetime of a single magnet we must consider the problem of "wear out" of the magnets as a system including such phenomena as the fault in the interconnecting leads that has been encountered in the Tevatron. To study this problem, one needs a few magnets (half-cell) operated for many more cycles than expected in the SSC $\left(10^{4}\right)$. Thus, $10^{5}$ magnetic-cycles on 10 magnets may expose a "wear out" problem and is actually a more stringent test of such a failure than $10^{4}$ cycles on 100 magnets.

As a result of the above considerations, the tests have been split into short string tests and long string tests. It must be emphasized that technical and economic limitations of ramping and quenching a string as long as 1 percent of the ring makes this split imperative if the "wear out" problem is to be studied. Thus, $10^{5}$ magnetic cycles, which should be obtainable on the short string, is equivalent to ten times the number of cycles expected in the SSC during its 20-year life expectancy.

A similar consideration was involved in setting the criteria for the quench tests. During a 20-year period, fifty string quenches correspond to the expected "random" number of quenches in a "quench protection cell" of the SSC. However, so far, "random" quenches have not been experienced in the Tevatron. Instead the cause can, in each case, be traced to beam loss or refrigeration problems. Such problems are not random but cause repeated quenches in a few magnets. We take the position that such quenches can be eliminated after their cause is determined. However, many more than 50 quenches of some magnets may be encountered before the "fix" is accomplished. Thus, for the short string tests we propose 500 quenches as a test of "wear out."

Cooldown and thermal stressing were handled in the same manner. The field quality and orientation should be measured periodically during these tests.

However, we also recognize that a short string does not model very well the behavior of a magnet in the machine. In the real machine, the helium pressure and flow rate may be much more severe under quench conditions than would exist in a short string. Such high pressure turbulent flow may cause mechanical damage. Also, the transient electrical behavior may cause high voltages to exist under abnormal operating conditions in a long string that would not occur in the shorter test string. In addition, tests must be made of the installation procedures and of the mechanical and electrical behavior of the magnet interconnections.

For these reasons, we specify also a comprehensive series of long string tests. In these tests we try at least to equal the expected SSC cycles and exceed them where it is economically and time-wise feasible. In addition, we try to obtain information on incipient failures by operation at increased stress. For instance, by cooling the string to $3.5 \mathrm{~K}$ the current can be increased by 20 percent and the forces by 40 percent, or by using a high-voltage dc bias, the insulation can be abnormally stressed during quench conditions. In general, the short string and long string tests are complementary and are designed to give a high degree of confidence that the magnet will perform satisfactorily. It is worth noting that the tests proposed here would have uncovered all of the difficulties encountered in the Tevatron program. 


\section{B. Description of the Test Program}

A test program meeting the criteria given in Section III.A and other operational requirements will now be discussed. Several important assumptions associated with this program are as follows:

- All magnets and other components have been individually tested at the place of manufacture.

- There are several magnet test facilities available for the required tests on individual magnets.

- The list of suggested tests implies that main coils, trim coils, and spools are being tested as well as the associated ancillary equipment.

\section{Tests Associated with Installation}

Techniques for magnet installation, including handling and transport, placement, interconnection, vacuum checking, and repair must be developed and assessed. Procedures for survey and alignment (warm and cold) should be developed. The STF will also be used to develop techniques for identification and replacement of defective or destroyed components. This is especially important because it is possible that most of the magnetic devices in the SSC will be installed without cryogenic tests, implying a certain initial failure rate. In fact, methods for finding bad components will have to be perfected using the STF. Techniques for installation, calibration, and debugging of instrumentation should be explored.

\section{Tests Associated with Cryogenic Properties}

The Cryogenic testing has two primary goals. The first is very early evaluation to obtain data to determine if the magnet chosen is compatible with the SSC layout and spacing of the refrigerator. We need to test a 50 to 150 meter length to obtain quantities such as heat leak, flow impedances, etc. This testing must be done early even if it is done with magnets that do not meet all electrical criteria.

The second goal is to obtain detailed cryogenic data on the complete system. Cryogenic properties should be measured, including cooldown characteristics and verification of the heat load. The static and dynamic characteristics of the system should be recorded including quench recovery rate and behavior under vacuum, power line, and single valve failure. Magnet removal and replacement techniques should be verified. Magnet pressure drops should be measured. Behavior under various ramp rate conditions should be studied. The temperature of the magnet string should be varied by $\pm 1 \mathrm{~K}$. The ability to decrease the temperature by $1 \mathrm{~K}$ allows one to overstress the magnets mechanically during a life test. Local heat loads should be simulated. Heat leak as a function of insulating vacuum pressure and recooler performance and stability should be measured. Systemdependent phenomena such as 2-phase stability (for B-type magnets) or the interaction between the $5 \mathrm{~K}$ and $80 \mathrm{~K}$ systems (for A-type magnets) should be investigated. 


\section{Tests Associated with Power Supplies}

These tests include establishing the requirements and testing the performance of the quench detection and protection scheme, measuring the ramp rate performance, testing $\mathrm{dc}$ operation and power supply regulation, and verifying the mechanical and electrical design of the magnets with hipot and lifetime tests. The trim coils system should be tested as well as the power lead performance under ramping and full current dc operation. The effect of power supply trips should be studied.

\section{Short String Accelerated Tests}

A short string will consist of components comprising one-half cell. The following tests should be performed:

a. Repeated ramping to full operating current with a goal of $10^{5}$ cycles at a rate of $\sim 200$ seconds per cycle (see Figure III.3).

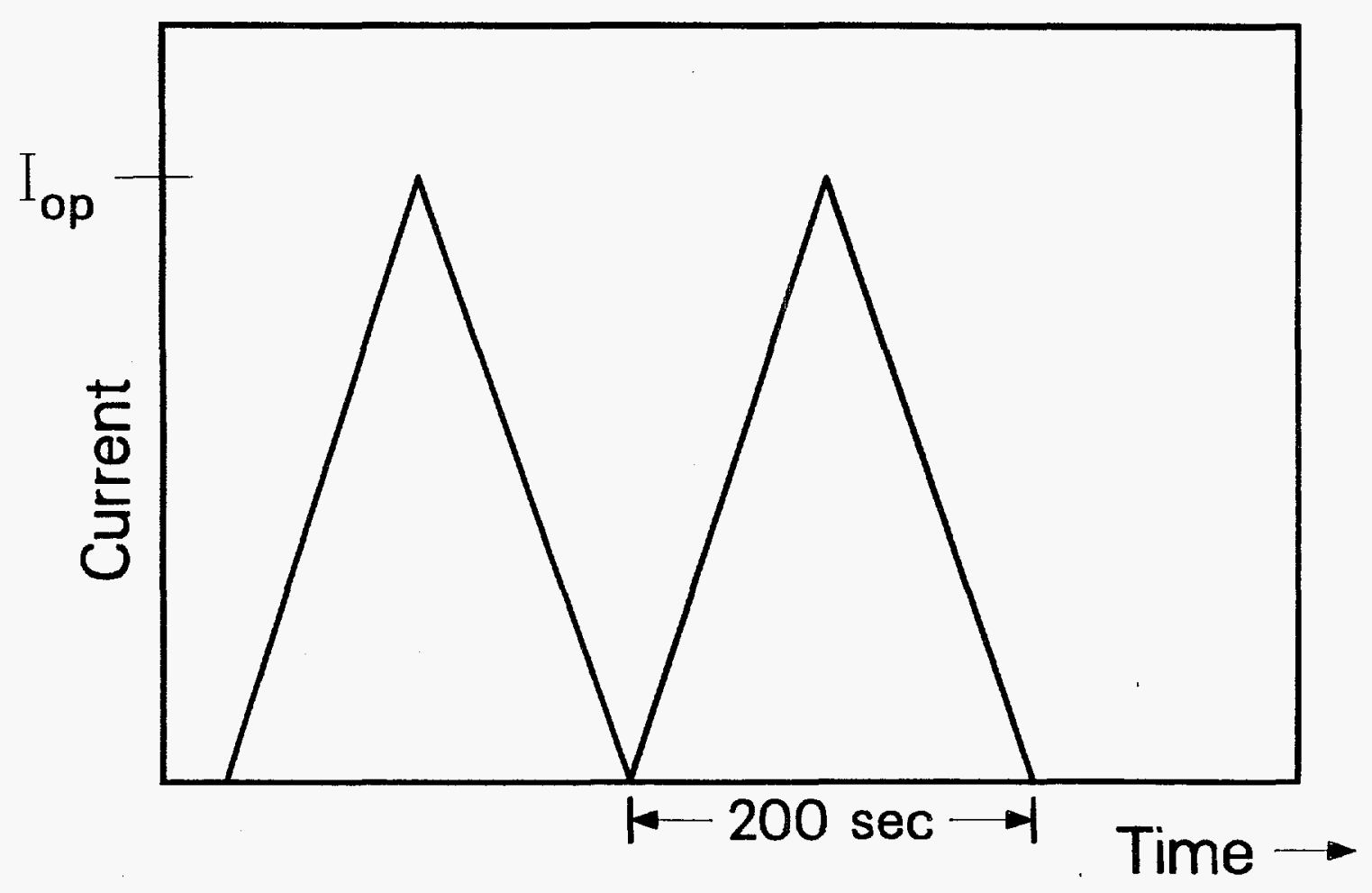

XCG 8410-13376

Fig. III.3 A Typical Cycle for the Short String Test. 
b. Investigation of the effect of the induced turn-to-turn voltage, while dumping into a resistive load.

c. One hundred (100) thermal cycles [ninety percent $(90 \%)$ of these should be between ambient and $80 \mathrm{~K}$, and ten percent $(10 \%)$ between ambient and $4.5 \mathrm{~K}$ ].

d. Five hundred (500) induced quenches.

\section{Long String Accelerated Tests}

The long string will consist of a length of magnets and other components amounting to about $1 \%$ of the circumference of the SSC Main Ring. The following tests should be made:

a. Repeated ramping to full operating current at a rate of $\sim 15 \mathrm{~min} /$ cycle (See Figure III.4).

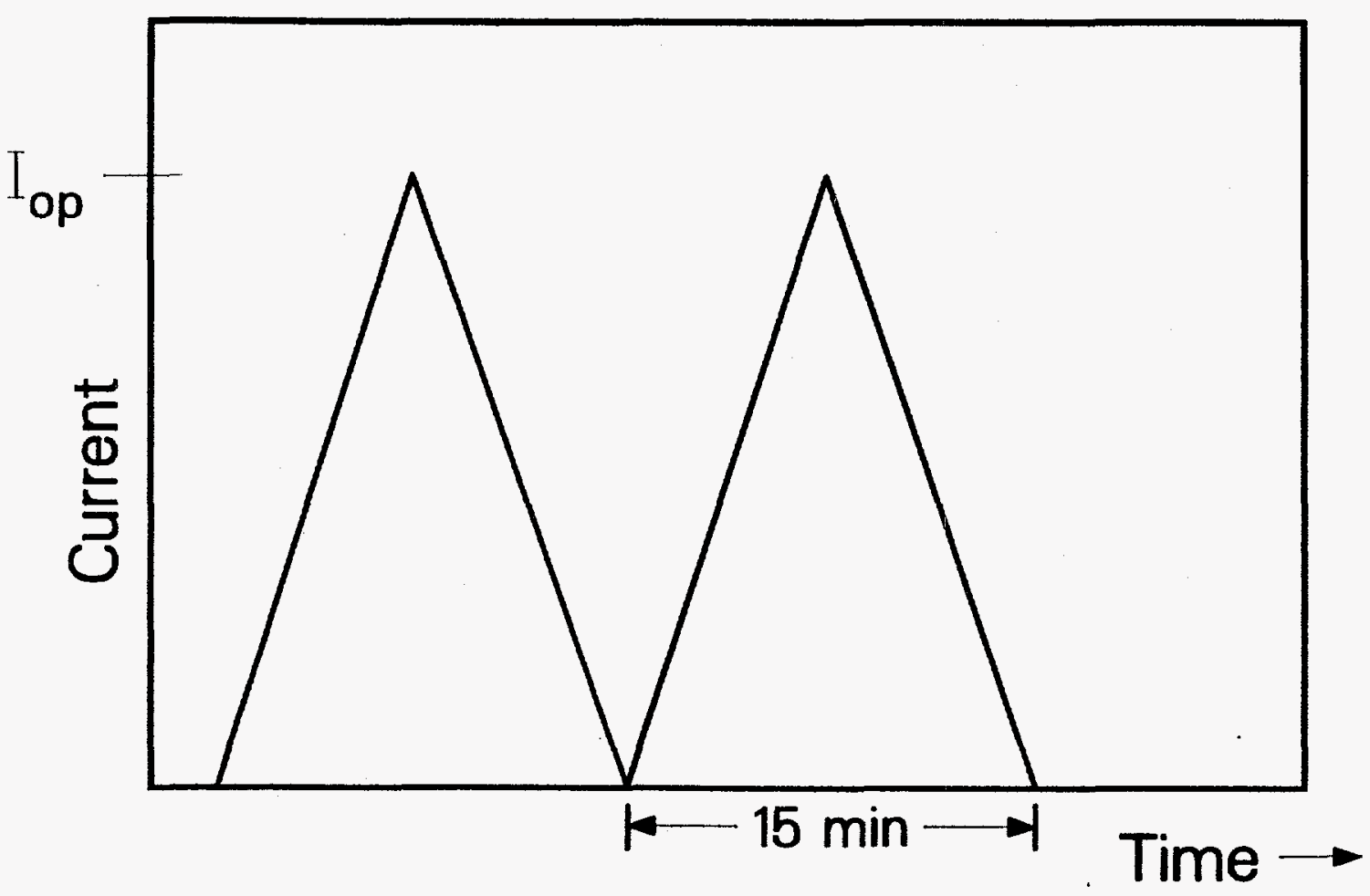

XCG $8410-13375$

Fig. III.4 A Typical Cycle For the Long String Tests.

b. Repeated ramping to 1.2 times the operating current. (This may be done at a temperature below the normal operating temperature.)

c. Ramping, quenching, and energy removal with voltage-to-ground greater than expected under fault conditions. 
d. Stable dc operation at full $20 \mathrm{TeV}$ current.

e. Ten full thermal cycles.

f. One hundred-fifty (150) half-cell quenches and approximately ten full-string quenches at operating current.

g. Probes from time to time of the peak current possible in the string (ramp-toquench). (We suggest about ten of these current ramps to quench.)

\section{Other Tests}

Tests should be conducted of the safety systems. These should include tests of the oxygen-deficiency-hazard safety procedures and systems. 


\section{Major Facilities Required}

The laboratory that is the site of the STF must provide a variety of facilities that are either purchased with SSC R\&D funds or are already available. These facilities are determined by the system tests described in Section III. The following is an attempt to specify facility requirements well enough to allow each candidate laboratory to develop a proposal for meeting them. It is divided into seven major categories: refrigeration, power supply, controls, quench systems, utilities, space, and miscellaneous.

\section{A. Refrigeration System}

A refrigerator (or refrigerators) capable of supporting the test program detailed in Section III should be provided. The specifications of the refrigerators given in Table IV.1 are determined by the following system test requirements:

\section{Quench Recovery}

For the long string test, after a quench of one "quench protection unit" (nominally one half-cell), the refrigerator systems should be capable of recooling the string to operating temperature and allowing ramping to maximum current in less than one hour. For the short string test the refrigerator should also be capable of recooling this string in one hour, but, in addition, the system should support tests that include multiple quenches separated by about one hour. 
Table IV.1

Cryogenic Requirements-Long String

$\left.\begin{array}{lccc}\hline \text { Mode } & \text { Type A } & \text { Type B } & \text { Type C } \\ \hline \text { Cooldown: } & 103 \\ \text { Flow (g/sec) } & 80\end{array}\right\} \&\left\{\begin{array}{ccc}63 & 100 & 36 \\ 20 & <20 & 80\end{array}\right\} \&\left\{\begin{array}{l}22 \\ 20 \\ T_{\text {in }}(\mathrm{K})\end{array}\right.$

Steady State Heat Load:

$\begin{array}{lccc}\text { Liquefaction }(\mathrm{g} / \mathrm{sec}) & 1.9 & 1.6 & 1.2 \\ \text { Refrigeration }(\mathrm{W}) & 572 & 425 & 340\end{array}$

Quench Recovery (1 hr):

\begin{tabular}{rrrrr} 
Refrigeration (W) & $2650^{*}$ & $1720^{*}$ & $390^{*}+$ High Flow \\
\hline & Cryogenic Requirements-Short String & \\
Mode & Type A & Type B & Type C \\
\hline
\end{tabular}

Cooldown:

Flow $(\mathrm{g} / \mathrm{sec})$

$\mathrm{T}_{\text {in }}(\mathrm{K})$

Time (Days)

Steady State Heat Load:

Liquefaction $(\mathrm{g} / \mathrm{sec})$

Refrigeration (W)

Quench Recovery (1 hr):

Refrigeration (W) $2650^{*}$
0.65

46

48

$42-1 / 2$
23

$\leqslant 80$

1 


\section{Ramping}

The maximum ramp rate for the long string without quenching is zero to maximum current and back to zero again in 15 minutes (see Fig. III.4). The refrigerator should be capable of supporting this test on a continuing basis.

\section{Operating Temperature}

Several magnet and string tests will be carried out at special operating temperatures. For example, high fields and currents, which result in high stress, can be imposed on the magnets at lower than normal operating temperature. The refrigerator for the long string is required to operate at any temperature between 3.5 and $5.5 \mathrm{~K}$ although other requirements are relaxed for these special tests.

\section{B. Power Supply System}

The requirements for magnet types $\mathbf{A}$ and $\mathbf{B}$ are quite similar and are distinct from those of type C. The laboratory at which the string test(s) is to be carried out should specify the power supply system for each of the three designs and await the final decision on the magnet type before ordering a power supply. This assumes that available power supplies will be used for initial tests. The approximate characteristics of the three magnet types and their power supplies are given in Table IV.2.

Table IV.2

Characteristics of the Three Magnet Systems and Their Power Supplies

\begin{tabular}{lccc}
\hline Long String & A & B & C \\
\hline Inductance (H) & 5.4 & 7 & 0.28 \\
Current (operating) (A) & 6000 & 6000 & 10,000 \\
Current (shunt, type C only) (A) & - & - & 5500 \\
Ramp time (minimum) (s) & 450 & 450 & 450 \\
Resistance ( $($ ) & 0.01 & 0.01 & 0.005 \\
Series SCR's & 4 & 4 & 2 \\
Voltage (ramping) (V) & 72 & 93 & 6 \\
Voltage (resistive) (V) & 60 & 60 & 50 \\
Voltage (SCR's) (V) & 8 & 8 & 4 \\
Voltage (total) (V) & 140 & 161 & 60 \\
Power Supply & & & \\
Voltage (V) & 220 & 220 & $120 / 60^{*}$ \\
Current (A) & 7000 & 7000 & $12000 / 6000^{*}$ \\
\hline
\end{tabular}

*Two supplies are needed for magnet type $C$. 
The power supply system will provide not only a test of the actual operating conditions of the magnet string, but also of the control and data acquisition systems. For these reasons the power supply control and regulation must include transductors for sensing at the part in $10^{4}$ level and must have control electronics capable of regulating to this level.

Because the magnet strings will be quenched, the power supplies must be compatible with the quench detection and protection systems.

\section{Magnet Types A and B}

The expected operating current is $6000 \mathrm{~A}$ and the maximum required charging voltage is $161 \mathrm{~V}$ for a 7-1/2 minute ramp of a long string. The power supply required for these two systems is arbitrarily set higher, at $7000 \mathrm{~A}$ and $220 \mathrm{~V}$, about $1.5 \mathrm{MVA}$, to accommodate special test conditions.

\section{Magnet Type $\mathrm{C}$}

The system consists of a $10 \mathrm{kA}$ main circuit with a $5.5 \mathrm{kA}$ shunt circuit. The maximum current for the system is set at $12,000 \mathrm{~A}$ at $120 \mathrm{~V}$. The shunt supply operates at a maximum current requirement of $6000 \mathrm{~A}$ at $60 \mathrm{~V}$.

\section{Trim Supplies}

In magnet systems $\mathrm{A}$ and $\mathrm{B}$ the quadrupoles are in series with the main magnets but may require a shunt power supply to adjust current to accommodate hysteretic effects. In magnet system C, a special power supply, $2500 \mathrm{~A}$ and about $100 \mathrm{~V}$, should be provided for the quadrupoles, which are independent of the dipoles. All three magnet systems have trim magnets at the end of each half-cell. Though some of these were included in the SSC Reference Design Study, the final details have not been determined. Nevertheless, a set of power supplies in the 50 to $500 \mathrm{~A}$ range will be required, and as a first guess these will be about the same, independent of magnet type. A special sextupole correction coil has been proposed for magnet type A and possibly B. These coils may be inductively-driven or may require an additional $\sim 100$ A power supply.

\section{Control, Logging, and Simulation System}

A control system should be provided to control and monitor the refrigerator, power supply, quench detection, quench protection, and all other systems. Computerized data logging with enough channels to $\log$ and store at an appropriate rate all parameters of interest (e.g., current as a function of time, temperature distribution, etc.) will be required. This system might include, for example, a VAX $11 / 780$ with $\approx 300$ Mbytes of disk storage, color graphics, hardcopy capability, and an appropriate amount of distributed intelligence along the test string. In addition, the system should include a high bandwidth data acquisition system with at least $10 \mathrm{kHz}$ capability on some channels and a recovery rate of $\sim 1$ $\mathrm{Mbyte} / \mathrm{sec}$ from buffers that are loaded during quenches. Further, good logging and archiving capabilities should be available for the analysis of magnet and string performance. 


\section{Quench Detection and Protection System}

The quench detection system should be capable of detecting a normal region in the superconductor on a timescale that will allow the energy to be extracted without damage. Detecting a quench should trigger a protection system that extracts the energy from the full string by opening SCR switches and backup dc breakers that put dump resistors in the circuit. In addition, the protection system may include heaters in the magnets. If so, the heaters in the magnets of the half-cell including the quenched coil should all be energized to cause the entire half-cell to quench. For the purpose of estimating, the following elements are included:

\section{For Magnets A and B}

\section{Quench Detection}

A monitor system capable of following 40 isolated differential channels plus miscellaneous analog and status signals. It should also be able to control power supplies, dump bypass circuits, and heater firing units.

\section{Dump Switches}

The following switches will be required:

1. A SCR switch capable of conducting $7000 \mathrm{~A}$ and designed to withstand $2000 \mathrm{~V}$ when opening.

2. A backup dc breaker with same capability as the SCR switch.

3. Dump control electronics.

\section{Heater Firing Units}

Two 600 Joule channels per half-cell per string, $\sim 40$ channels, addressable as pairs.

\section{For Magnet B (only)}

\section{Magnet Bypass Circuit}

Magnet type B requires the following components:

1. Two parallel cable paths of approximately $350 \mathrm{MCM}$ for each bus of each string.

2. Two independent bypass SCR circuits per half cell per bus per string; each circuit should be capable of passing $7000 \mathrm{~A}$ with a $30 \mathrm{~s}$ decay time constant (735 MIITS).

3. Two independent bypass SCR control electronics systems. 


\section{For Magnet C}

This system is only passively protected. Dump resistors, SCR switches and backup dc breakers capable of operating with currents of $6000 \mathrm{~A}$ and 12,000 A and appropriate dump control electronics are the only requirements.

\section{E. Utilities}

The following utilities should be provided:

1. Power and cooling water necessary for the refrigeration system.

2. $110 / 208 / 480 \mathrm{~V}$ power along the string capable of supporting magnet power supplies, vacuum pumps, heliarc welding, hand tools and miscellaneous.

3. For the magnet type $B$, there is an additional electric power requirement for magnet warm-up. To warm-up a section requires $100 \mathrm{~kW}$ at $480 \mathrm{~V}$. This power should be available in the form of welding outlets at $\sim 200 \mathrm{~m}$ intervals in the tunnel.

4. Heating, lighting, ventilation and air-conditioning of all spaces as appropriate.

\section{F. Space}

The space available for the long string test should, in every way possible, simulate that expected in the SSC. For example, the string should be in a "mock tunnel" enclosure that, in terms of limitation of motion, is identical to that of the final tunnel. In addition, the relative humidity in the SSC tunnel is of concern and should also be simulated. If the radius of curvature for the string test is different from that of the SSC then the "mock tunnel" size should be adjusted to provide the expected limits for magnet installation, repair, and removal in the SSC.

The general space requirements are:

1. A magnet string enclosure $1.2 \mathrm{~km}$ long and about $3 \mathrm{~m}$ wide. The temperature should be maintained between 55 and $90^{\circ} \mathrm{F}$ and the relative humidity less than $80 \%$.

2. A control room of $\sim 600 \mathrm{ft}^{2}$.

3. Local office space for dedicated and temporary staff; 15 offices of $100 \mathrm{ft}^{2}$; at least 5 of these should be at the test site.

4. Local technician work area of $\sim 1200 \mathrm{ft}^{2}$.

5. Adequate parking space. 


\section{G. Miscellaneous}

1. Nearby machine shop facilities under laboratory control.

2. Appropriate furniture and other office and laboratory equipment.

3. Sanitary facilities.

4. Two pickup or panel trucks.

5. A personnel and interlock safety system meeting the requirements of the laboratory. 



\section{Alternative Locations of the System Test Facility}

The complete responses of BNL and FNAL to the specifications for the STF are given in Appendices D and E, respectively. These appendices have been reviewed for completeness by the Task Force, but are presented here as unedited responses from the two laboratories. Summary descriptions of these laboratory responses are given in Sections V.A and V.B. Tables in V.C allow a comparison of total construction cost, total operating cost, and total manpower requirements for the two laboratories. Additional tables give break-downs of the cost and manpower data into the two major categories: Long String Facility and Short String Facility. Further tables show the cost of civil construction, cryogenic capabilities, cryogenic equipment needed, power supply and quench protection hardware, and control systems costs. The summary descriptions and tables given in this section were provided by the laboratories along with their responses (Appendices $D$ and $E$ ) and are presented here in a form that allows easy reference.

\section{A. Summary of the BNL Response}

Brookhaven proposes to accomplish the goals of the test program specified in Section III for both the Long String and Short String Facilities in the following way:

\section{Long String Facility}

The magnet system would be housed in the empty CBA tunnel. It would be cooled by the R\&D refrigerator already located in the tunnel, operating "stand-alone." For operation in the event of much higher heat load than calculated, this refrigerator could be aided by injection of liquid helium in the FNAL "satellite" mode. If needed, this liquid would be stored in a large rented dewar which would be filled when necessary by operating the RHIC $25 \mathrm{~kW}$ refrigerator at full capacity (and high efficiency). For cooldown of the entire string, the RHIC refrigerator would also be run at full capacity, accomplishing this in 1.2 days. 
The Collider Center building would be used to house STF staff including visitors from other laboratories, provide technician work areas, etc., in very close proximity to the magnet string. It could also provide sumptuous space for the future development of final SSC control systems if that were desired.

Two CBA power supplies are available to get early powered tests on the string underway. These would have to be replaced by a new supply in order to ramp the complete string at the maximum rate. This power supply would be purchased only after the magnet type has been chosen. Some switch gear and other smaller items are also available.

The control and data acquisition systems will be formed around an existing process control system purchased originally for cryogenic control for CBA, with a DECNET connection to the BNL On Line Data Facility (OLDF). The OLDF contains three VAX 11/780's, a very large memory and a great deal of peripheral equipment. It will serve for simulation and for making data correlations and other large-scale manipulations. With a modest amount of upgrading and programming, the process control system can serve all the required control and data acquisition functions.

\section{Short String Facility}

The magnet system would be housed in a large trench in Building 902 and would be cooled by the MAGCOOL refrigerator. This $1580 \mathrm{~W}$ refrigerator, located very near the trench, will be capable of the required rapid quench recovery. It can be devoted exclusively to this purpose, since the nearby PAT refrigerator will be used for production testing of magnets. Building 902 also contains office and lab space if some addition to that provided in the Collider Center is necessary.

If magnet type A or B is chosen, a third CBA power supply and other equipment is available for powering the string, and space is available in Building 902 to house it. A magnet $C$ short string would require new power supplies, but these would be available before testing could begin.

Cryogenic control and data acquisition would be managed through the MAGCOOL process control system. This would require a very small amount of programming and no additional hardware. Magnetic field and quench data would be collected by the existing magnetic measurement system. These two systems could easily be connected to each other, and if this combination is not sufficient for desired data manipulation, it could also be connected to the OLDF through a DECNET link.

\section{B. Summary of the FNAL Response}

Fermilab proposes to support the Long String Facility and the Short String Facility as independent facilities. The long string would be housed in a mock-up tunnel enclosure to be constructed on top of an existing roadbed (Kautz Road). The head end of this facility would be built right against one of the Saver satellite refrigerator buildings at E4, which would be expanded to house an additional satellite refrigerator. Two of the Saver compressors nearby at FO would be valved to permit dedicated support for this facility. With the exceptions noted in the summary tables this plant, in conjunction with the Central Helium 
Liquifier, could carry out the long string test program. In addition to the $1.2 \mathrm{~km}$ long mock tunnel enclosure, an appropriate "Head House" to house power supplies and technician space, as well as "Portakamps" to house control facilities and office space would be provided to meet the specifications. Power supplies would be provided initially from Fermilab spares, and augmented as the test string grows. Quench protection and power excitation would be provided initially by available Saver-style electronics, and again these would be augmented as the test string grows to the full $1 \%$ of SSC circumference. The initial test site controls for the long string could be provided immediately by attaching to the Saver serial link and using available Saver instrumentation. Complete console control would be provided by installing a standard ACNET console in the control portakamp.

The Short String Facility would be an adjunct to the existing Fermilab Magnet Test Facility (MTF). The existing Fermilab MTF refrigerator-compressor complex, augmented by a $20 \mathrm{~K}$ gas recovery system, would be used to provide the necessary cryogenic capability. The location of the short string at the MTF will also permit certain magnetic measurements to be made in the short string components during life tests, a procedure recommended in the STF specifications. Existing power supplies at the MTF should be sufficient for these tests, but could be augmented if required. The existing MTF data recovery/data reduction system would be used; existing plans for additions to the networking capability of that system will also be useful for transfer of data files.

The Fermilab proposal is characterized by relatively straightforward and inexpensive civil construction of physical plant, utilization of existing refrigeration equipment with slight augmentation of recovery capacity, and an extremely flexible approach to test control and data acquisition. This system will easily adapt to become an umbrella for the development of other SSC systems, such as quench protection, local area networks, and the study of integrated distributed processor support. The impact of this program on both the TeV I and TeV II programs has been evaluated and is believed to be minimal, or perhaps zero. The operation of the short string at the MTF can be scheduled satisfactorily in conjunction with other testing projects.

\section{Summary Tables from BNL/FNAL}

Tables summarizing the detailed information given in the responses of BNL and FNAL (Appendices D and E) to the specifications for the STF are shown in the following pages. For ease of comparison, the corresponding tables from the two laboratories are, in each case, shown on facing pages with BNL tables on the left side and FNAL tables on the right side. Conclusions based on these tables and other findings of the Task Force are presented in Section VI. 


\section{Brookhoven}

\section{CONSTRUCTION COST ( K \$) - TOTAL}

\begin{tabular}{|c|c|c|c|c|c|c|c|c|c|c|c|c|c|c|c|c|c|c|c|c|c|c|c|c|}
\hline & \multicolumn{6}{|c|}{ FY'85 } & \multicolumn{6}{|c|}{ FY '86 } & \multicolumn{6}{|c|}{ FY' 87} & \multicolumn{6}{|c|}{ FY' 88} \\
\hline & \multicolumn{2}{|c|}{ TYPEA } & \multicolumn{2}{|c|}{ TYPE B } & \multicolumn{2}{|c|}{ TYPEC } & \multicolumn{2}{|c|}{ TYPEA } & \multicolumn{2}{|c|}{ TYPE B } & \multicolumn{2}{|c|}{ TYPEC } & \multicolumn{2}{|c|}{ TYPE A } & \multicolumn{2}{|c|}{ TYPE $\mathbf{B}$} & \multicolumn{2}{|c|}{ TYPE C } & \multicolumn{2}{|c|}{ TYPEA } & \multicolumn{2}{|c|}{ TYPE $B$} & \multicolumn{2}{|c|}{ TYPE C } \\
\hline & M\&S & SWF & MBS & SWF & $M A S$ & SWF & $M \& S$ & SWF & mas & SWF & Mas & SiVF & Has & SWF & mas & SwF & Mas & SWF & Mas & SWF & MAS & SWF & 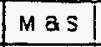 & SWF \\
\hline $\begin{array}{l}\text { 1. CIVIL } \\
\text { CONSTRUCTION }\end{array}$ & 100.0 & 32.0 & 100.0 & 32.0 & 100.0 & 32.0 & & 20.0 & & 20.0 & & 20.0 & & & & & & & & & & & & \\
\hline 2. CRYOGENICS & 110.0 & 100.0 & 110.0 & 100.0 & 110.0 & 100.0 & 178.0 & 352,0 & 163,0 & 352.0 & 154.0 & 352.0 & & & & & & & & & & & & \\
\hline 3. POWER SUPPLY Q QP & 95.0 & 300.0 & 95.0 & 300.0 & 60.0 & 300.0 & 473.0 & 550.0 & 548.0 & 550.0 & 878.0 & 550.0 & & & & & & & & & & & & \\
\hline $\begin{array}{l}\text { 4. CONTROLS AND DATA } \\
\therefore \text { COLLECTION } \\
\because \ldots\end{array}$ & 117:0 & $1 \cdot 10.0$ & $117: 0$ & 110.0 & 117.0 & 110.0 & 105.0 & 340.0 & 105.0 & 340.0 & 105.0 & 340.0 & & & & & & & & & & & & \\
\hline 5. SAFETY & $15: 0$ & 6,0 & 15,0 & 6.0 & 15.0 & 6.0 & & 12.0 & & 12.0 & & 12.0 & & & & & & & & & & & - & \\
\hline $\begin{array}{l}\text { 6. INSTALLATION } \\
\text { MECHANICAL } \\
\text { ELECTRICAL }\end{array}$ & $\cdots$ & 30.0 & & 30.0 & & 30.0 & 95.0 & 270.0 & 95.0 & 270.0 & 50.0 & 134.0 & & 90.0 & & 90.0 & & 46.0 & & & & & & \\
\hline 7. TEST COORDINATION & -- & & & & & & & & & & & & & & & & & & & & & & & \\
\hline 8. MISCELLANEOUS & 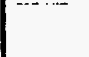 & & & & & & & & & & & & & & & & & & & & & & & \\
\hline 9. CRYOGENIC FLUIDS & & & & & & & & & & & & & & & & & & & & & & & & \\
\hline 10. POWER & & & & & & & & & & & & & & & & & & & & & & & & \\
\hline 11. TOTALS & 437.0 & $\$ 78.0$ & 437.0 & 578.0 & 402.0 & 578.0 & 851.0 & $2544 . n$ & 911.0 & 1544.0 & $|1187.0|$ & 408,0 & & 90.0 & & 90.0 & & 46.0 & & & & & & \\
\hline 12. TOTALS & 1015. & & 1015.0 & & 980.0 & & 239 & & 24 & 155 & 25 & 995 & 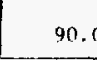 & & 90. & & 46 & & & & & & & \\
\hline & & & & & & & & $E: D C$ & NOT & NC & $G \& A$ & & & & & & ND TO & ALS & 350 & & & 60 & 362 & \\
\hline
\end{tabular}




\begin{tabular}{|c|c|c|c|c|c|c|c|c|c|c|c|c|c|c|c|c|c|c|c|c|c|c|c|c|}
\hline & \multicolumn{6}{|c|}{ FY' 85} & \multicolumn{6}{|c|}{ FY ' 86} & \multicolumn{6}{|c|}{ FY 'B7 } & \multicolumn{6}{|c|}{ FY ${ }^{\prime} 88$} \\
\hline & \multicolumn{2}{|c|}{ TYPEA } & \multicolumn{2}{|c|}{ TrPE a } & \multicolumn{2}{|c|}{ TYPEC } & \multicolumn{2}{|c|}{ TYPE A } & \multicolumn{2}{|c|}{ TYPE } & \multicolumn{2}{|c|}{ TYPE C } & \multicolumn{2}{|c|}{ IYPE A } & \multicolumn{2}{|c|}{ TYPE B } & \multicolumn{2}{|c|}{ TYPE C } & \multicolumn{2}{|c|}{ TYPEA } & \multicolumn{2}{|c|}{ TYPE $\mathrm{B}$} & \multicolumn{2}{|c|}{ TYPE C } \\
\hline & $M Q S$ & SWF & MOS & SWF & MAS & SWF & mas & $S W F$ & Mas & SWf & Mas & $5 W F$ & Mas & SWF & Mas & SWF & Mas & swF & Mas & SWF & Mas & Swf & MES & $\overline{\text { swF }}$ \\
\hline $\begin{array}{l}\text { 1. CIVIL } \\
\text { CONSTRUCTION }\end{array}$ & 166 & & 166 & & 166 & & 542 & & 542 & & 534 & & & & & & 30 & & & & & & & \\
\hline 2. CRYOGENICS & 130 & 200 & 130 & 200 & 130 & 200 & 105 & 230 & 105 & 230 & 105 & 230 & & & & & & & & & & & & \\
\hline $\begin{array}{l}\text { 3. POWER SUPPLY } Q \text { QPM } \\
\text {-_ (SOFTWARE) }\end{array}$ & 106 & $\begin{array}{l}50 \\
30\end{array}$ & 122 & $\begin{array}{l}70 \\
30\end{array}$ & 106 & $\begin{array}{l}50 \\
30\end{array}$ & 199 & $\begin{array}{r}100 \\
60\end{array}$ & 299 & $\begin{array}{r}190 \\
60\end{array}$ & 560 & $\begin{array}{r}140 \\
60\end{array}$ & & & & & & & & & & & & \\
\hline $\begin{array}{l}\text { 4. CONTROLS AND DATA } \\
\text { - COLLECTION } \\
\text { (SOFTWARE) }\end{array}$ & 82 & $\begin{array}{r}90 \\
60 \\
\end{array}$ & 82 & $\begin{array}{l}90 \\
60 \\
\end{array}$ & 82 & $\begin{array}{l}90 \\
60 \\
\end{array}$ & & 60 & & 60 & & 60 & & & & & & 1 & & & & : & & \\
\hline 5. SAFETY & 8 & 3 & 8 & 3 & 8 & 3 & 7 & 2 & 7 & 2 & 7 & 2 & & & & & & & & & & & & \\
\hline $\begin{array}{l}\text { 6. INSTALLATION } \\
\text { MECHANICAL } \\
\text { ELECTRICAL } \\
\end{array}$ & 25 & $\begin{array}{l}80 \\
40 \\
\end{array}$ & 25 & $\begin{array}{l}80 \\
40 \\
\end{array}$ & 50 & $\begin{array}{l}80 \\
40\end{array}$ & 125 & $\begin{array}{r}120 \\
60\end{array}$ & 125 & $\begin{array}{r}120 \\
60\end{array}$ & 200 & $\begin{array}{r}120 \\
60\end{array}$ & & & & & & & & & & & & \\
\hline 7. TEST COOROINATION & & 120 & & 120 & & 120 & & 30 & & 30 & & 30 & & & & & & & & & & & & \\
\hline Q MISCELLANEOUS & -25 & & 25 & & 25 & & 20 & & 20 & & 20 & & & & & & & & & & & & & \\
\hline 9. CRYOGENIC FLUIDS & 38 & & 20 & & 10 & & 3 & & 1 & & 1 & & & & & & & & & & & & & \\
\hline \multicolumn{25}{|l|}{ 10. POWER } \\
\hline II TOTALS & 580 & 673 & 578 & 693 & 577 & 673 & 1001 & 662 & 1099 & 752 & 1427 & 702 & & & & & 30 & & & & & & & \\
\hline 12 TOTALS & \multicolumn{2}{|c|}{1253} & \multicolumn{2}{|c|}{1271} & \multicolumn{2}{|c|}{1250} & \multicolumn{2}{|c|}{1663} & \multicolumn{4}{|c|}{2129} & & & & & \multicolumn{2}{|c|}{30} & & & & & & \\
\hline & & & & & & & & $E:$ & 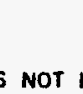 & 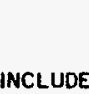 & OA & & & & & & ID T & 5 & 29 & & 312 & & 340 & \\
\hline
\end{tabular}


$\mathrm{v}-2$

CONSTRUCTION COST (K \$)-LONG STRING

\begin{tabular}{|c|c|c|c|c|c|c|c|c|c|c|c|c|c|c|c|c|c|c|c|c|c|c|c|c|}
\hline & \multicolumn{6}{|c|}{ FY' 85} & \multicolumn{6}{|c|}{$F Y^{\prime} 86$} & \multicolumn{6}{|c|}{ FY' 87} & \multicolumn{6}{|c|}{ FY' 88} \\
\hline & \multicolumn{2}{|c|}{ TYPEA } & \multicolumn{2}{|c|}{ TYPE B } & \multicolumn{2}{|c|}{ TYPE C } & \multicolumn{2}{|c|}{ TYPEA } & \multicolumn{2}{|c|}{ TYPE B } & \multicolumn{2}{|c|}{ TYPE C } & \multicolumn{2}{|c|}{ TYPE A } & \multicolumn{2}{|c|}{ TYPE B } & \multicolumn{2}{|c|}{ TYPEC } & \multicolumn{2}{|c|}{ TYPEA } & \multicolumn{2}{|c|}{ TYPE B } & \multicolumn{2}{|c|}{ TYPE C } \\
\hline & Mas & SWF & Mas & $5 W F$ & mas & SWF & mas & $S W F$ & MQS & SWF & MBS & SWF & Mas & SWF & MAS & SWF & MBS & SWF & mas & SWF & mas & SWF & Mas & SWF \\
\hline $\begin{array}{l}\text { 1. CIVIL } \\
\text { CONSTRUCTION }\end{array}$ & 100.0 & 32.0 & 100.0 & 32.9 & 100.0 & 32.0 & & 20.0 & & 20,0 & & 20.0 & & & & & & & & & & & & \\
\hline 2. CRYOGENICS & 10.0 & 50.0 & 10.0 & 50.0 & 10.0 & 50.0 & 135.0 & 240.0 & 120.0 & 240.0 & $[1] .00]$ & 240.0 & & & & & & & & & & & & \\
\hline 3. POWER SUPPLY $Q$ QF & 60.0 & $\mid 198.0$ & 60.0 & 198.0 & 40.0 & $\mid 198.0$ & 400.0 & $|366.0|$ & 470.0 & 366.0 & 755.033 & 366.0 & & & & & & & & & & & & \\
\hline $\begin{array}{l}\text { 4. CONTROLS AND DATA } \\
\text { COLLECTION } \\
\end{array}$ & 1.17 .0 & 78.0 & 117.0 & $: 78.0$ & $|117.0|$ & 78,0 & 105.0 & $\mid 288.0$ & | 105.0 & 288.0 & 105.02 & 288.0 & & & & & & & & & & & & \\
\hline 5. SAFETY & 15.0 & 6.0 & is.0 & 6.0 & 15.0 & 6.0 & & 8.0 & & 8.0 & & 8.0 & & & & & & & & & & & & \\
\hline \begin{tabular}{l} 
6. INSTALLATION \\
\hdashline MECHANICAL \\
ELECTRICAL. \\
\end{tabular} & $\cdots$ & 24.0 & & $\begin{array}{r}24.0 \\
-\end{array}$ & $\ldots$ & 24.0 & 85.0 & $242,0 \mid$ & 85.0 & 242.0 & 45.011 & 120.0 & & 76.0 & & 76.0 & & .42 .0 & & & & & & \\
\hline 7. TEST COORDINATION & 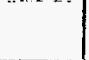 & & & & & & & & & & & & & & & & & & & & & & & \\
\hline B. MISCELLANEOUS & & & & & & & & & & & & & & & & & & & & & & & & \\
\hline 9. CRYOGENIC FLUIDS & & & & & & & & & & & & & & & & & & & & & & & & \\
\hline 10. POWER & & & & & & & & & & & & & & & & & & & & & & & & \\
\hline II. TOtAls & 302.0 & 388.0 & 302.0 & 388.0 & 282.0 & 388.0 & 725.0 & 1164.0 & 780.0 .1 & 1164.018 & 1016.0 plo & .042 .0 & & 76.0 & & 76.0 & & 42.0 & & & & & & \\
\hline 12. TOTALS & \multicolumn{2}{|c|}{690.0} & \multicolumn{2}{|c|}{690.0} & \multicolumn{2}{|c|}{670.0} & \multicolumn{2}{|c|}{1889.0} & \multicolumn{2}{|c|}{1944.0} & \multicolumn{2}{|c|}{2058.0} & \multicolumn{2}{|c|}{76.0} & \multicolumn{2}{|c|}{76.0} & \multicolumn{2}{|c|}{42.0} & & & & & & \\
\hline & & & & & & & & $\mathrm{E}$ : & NOT & & & & & & & & D T & & 2655 & & 271 & & 27 & 70 \\
\hline
\end{tabular}




\begin{tabular}{|c|c|c|c|c|c|c|c|c|c|c|c|c|c|c|c|c|c|c|c|c|c|c|c|c|}
\hline & \multicolumn{6}{|c|}{ FY ' 85} & \multicolumn{6}{|c|}{ FY '86 } & \multicolumn{6}{|c|}{ FY' 87} & \multicolumn{6}{|c|}{ FY ' 88} \\
\hline & \multicolumn{2}{|c|}{ TYPEA } & \multicolumn{2}{|c|}{ TYPE $\mathrm{B}$} & \multicolumn{2}{|c|}{ TYPEC } & \multicolumn{2}{|c|}{ TYPE A } & \multicolumn{2}{|c|}{ TYPE B } & \multicolumn{2}{|c|}{ TYPEC } & \multicolumn{2}{|c|}{ TYPEA } & \multicolumn{2}{|c|}{ TYPE 8} & \multicolumn{2}{|c|}{ TYPE C } & \multicolumn{2}{|c|}{ TYPEA } & \multicolumn{2}{|c|}{ TYPE $B$} & \multicolumn{2}{|c|}{ TYPE $C$} \\
\hline & MQS & SWF & M B S & SWF & MAS & SWF & Mas & sWF & MBS & $S W F$ & mas & $5 W F$ & MAS : & SNF & Mas & swF & MBS & SNF & Mas & $5 \% F$ & $M A \leq 1$ & SWF & MAS & SWF \\
\hline 2. CRYOGENICS & 35 & 100 & 35 & 100 & 35 & 100 & 100 & 110 & 100 & 110 & 100 & 110 & & & & & & & & & & & & \\
\hline $\begin{array}{l}\text { 4. CONTROLS AND DATA } \\
\text { COLLECTION } \\
\text { (SOFTWARE) }\end{array}$ & 82 & $\begin{array}{l}90 \\
60\end{array}$ & 82 & $\begin{array}{l}90 \\
60\end{array}$ & 82 & $\begin{array}{l}90 \\
60\end{array}$ & & & & & & & & & & & & & & & & & & \\
\hline 5. SAFETY & 8. & 3 & 8 & 3 & 8 & 3 & 7 & 2 & 7 & 2 & 7 & 2 & & & & & & & & & & & & \\
\hline $\begin{array}{l}\text { 6. INSTALLATION } \\
\text { MECHANICAL } \\
\text { ELECTRICAL }\end{array}$ & 25 & $\begin{array}{l}80 \\
40\end{array}$ & 25 & $\begin{array}{l}80 \\
40\end{array}$ & 50 & $\begin{array}{l}80 \\
40\end{array}$ & 100 & $\begin{array}{l}80 \\
40\end{array}$ & 100 & $\begin{array}{l}80 \\
40\end{array}$ & 150 & $\begin{array}{l}80 \\
40\end{array}$ & & & & & & & & & & & & \\
\hline 7. TEST COORDINATION & & 120 & & 120 & & 120 & & & & & & & & & & & & & & & & & & \\
\hline 8 MISCELLANEOUS & 25 & & $25^{\circ}$ & & 25 & & & & & & & & & & & & & & & & & & & \\
\hline 9. CAYOGENIC FLUIDS & 38 & & 20 & & 10 & & & & & & & & & & & & & & & & & & & \\
\hline \multicolumn{25}{|l|}{ 10. POWEA } \\
\hline II. TOTALS & 485 & 573 & 483 & 593 & 482 & 573 & 928 & 312 & 1028 & 402 & 1331 & 352 & & & & & 30 & & & & & & & \\
\hline 12 TOTALS & \multicolumn{2}{|c|}{1058} & \multicolumn{2}{|c|}{1076} & \multicolumn{2}{|c|}{1055} & \multicolumn{4}{|c|}{1430} & \multicolumn{2}{|c|}{1683} & & & & & \multicolumn{2}{|l|}{30} & & & & & & \\
\hline & & & & & & & NOT & IE $: D$ & TI & Lit & 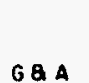 & & & & & & AND TO & ALS & & 98 & 25 & & & 68 \\
\hline
\end{tabular}


v -3

\begin{tabular}{|c|c|c|c|c|c|c|c|c|c|c|c|c|c|c|c|c|c|c|c|c|c|c|c|c|}
\hline & \multicolumn{6}{|c|}{ FY' 85} & \multicolumn{6}{|c|}{ FY '86 } & \multicolumn{6}{|c|}{ FY' 87} & \multicolumn{6}{|c|}{ FY' 88} \\
\hline & \multicolumn{2}{|c|}{ TYPE A } & \multicolumn{2}{|c|}{ TYPE B } & \multicolumn{2}{|c|}{ TYPEC } & \multicolumn{2}{|c|}{ TYPE A } & \multicolumn{2}{|c|}{ TYPE B } & \multicolumn{2}{|c|}{ TYPEC } & \multicolumn{2}{|c|}{ TYPE A } & \multicolumn{2}{|c|}{ TYPE B } & \multicolumn{2}{|c|}{ TYPE C } & \multicolumn{2}{|c|}{ TYPEA } & \multicolumn{2}{|c|}{ TYPE B } & \multicolumn{2}{|c|}{ TYPE C } \\
\hline & 1485 & SWF & MBS & SWF & Mas & SWF & M8s & SWF & $M B S$ & SWF & mas & SWF & Mas & SWF & MQS & SWF & MBS & SWF & mas & SWF & MAS & SWF & in 85 & SwF \\
\hline \multicolumn{25}{|l|}{$\begin{array}{l}\text { 1. CIVIL } \\
\text { CONSTRUCTION }\end{array}$} \\
\hline 2. CRYOGENICS & 100.0 & 50.0 & 100.0 & 50.0 & 100.0 & 50.0 & 43.0 & 112.0 & 43.0 & 112.0 & 43.0 & 112.0 & & & & & & & & & & & & \\
\hline \begin{tabular}{l} 
3. POWER SUPPLY Q QP \\
\hdashline$-\cdots$
\end{tabular} & $\begin{array}{c}35.0 \\
\cdots:\end{array}$ & 102.0 & 35.0 & 102.0 & 20.0 & 102.0 & 73.0 & 184.0 & 78.0 & 184.0 & 123.0 & 184.0 & - & & & & & & & & & & & \\
\hline $\begin{array}{l}\text { 4. CONTROLS AND DATA } \\
\because \text { COLLECTION } \\
\end{array}$ & 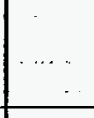 & 32.0 & & 32.0 & & 32.0 & & 52.0 & & 52.0 & & 52.0 & & & & & & & & & & & & \\
\hline 5. SAFETY & $\cdots$ & .... & $\therefore$ & . & & & & 4,0 & & 4.0 & & 4.0 & & & & & & & & & & & & \\
\hline $\begin{array}{l}\text { 6. INSTALLATION } \\
\text { MECHANICAL } \ldots\end{array}$ & $\cdots$ & 6.0 & & 6.0 & & 6.0 & 10.0 & 28.0 & 10.0 & 28.0 & 5.0 & 14.0 & & 14.0 & & 14.0 & & 4.0 & & & & & & \\
\hline \multicolumn{25}{|l|}{ 7. TEST COORDINATION } \\
\hline \multicolumn{25}{|l|}{ 8. MISCELLANEOUS } \\
\hline \multicolumn{25}{|l|}{ 9. CRYOGENIC FLUIDS } \\
\hline \multicolumn{25}{|l|}{ 10. POWER } \\
\hline II. TOTALS & 135.0 & 190.0 & 135.0 & 190.0 & 120.0 & 190,0 & 126.0 & 380.0 & 131.0 & 380.0 & 171.0 & 366.0 & & 14.0 & & 14.0 & & 4.0 & & & & & & \\
\hline 12. TOTALS & \multicolumn{2}{|c|}{325.0} & \multicolumn{2}{|c|}{325.0} & \multicolumn{2}{|c|}{310.0} & \multicolumn{2}{|c|}{506.0} & \multicolumn{2}{|c|}{511.0} & \multicolumn{2}{|c|}{537.0} & \multicolumn{2}{|c|}{14.0} & \multicolumn{2}{|c|}{14.0} & 4 & & & & & & & \\
\hline & & & & & & & & TE : DOES & S nor & INCLUDE & $G \& A$ & & & & & & ND TO & TALS & 845.0 & & 850. & & 851 & \\
\hline
\end{tabular}


Fermi TABLE $V-3$

CONSTRUCTION COST (K\$) -SHORT STRING

\begin{tabular}{|c|c|c|c|c|c|c|c|c|c|c|c|c|c|c|c|c|c|c|c|c|c|c|c|c|}
\hline & \multicolumn{6}{|c|}{ FY'B5 } & \multicolumn{6}{|c|}{ FY ' 86} & \multicolumn{6}{|c|}{ FY 187} & \multicolumn{6}{|c|}{ FY ' 88} \\
\hline & \multicolumn{2}{|c|}{ TYPEA } & \multicolumn{2}{|c|}{ TYPE B } & \multicolumn{2}{|c|}{ TYPEC } & \multicolumn{2}{|c|}{ TYPE A } & \multicolumn{2}{|c|}{ TYPE $\mathbf{B}$} & \multicolumn{2}{|c|}{ TYPE C } & \multicolumn{2}{|c|}{ TYPE A } & \multicolumn{2}{|c|}{ TYPE $\mathrm{B}$} & \multicolumn{2}{|c|}{ TYPE C } & \multicolumn{2}{|c|}{ TYPEA } & \multicolumn{2}{|c|}{ TYPE 8} & \multicolumn{2}{|c|}{ TYPE $C$} \\
\hline & mas & SWF & Mas & sWF & MES & SWF & MBS & SWF & MBS & SWF & mas & SNF & Mas & SWF & MAS & SWF & Mas! & SWf & Mas & SAf & Mas & SWF & MAS & SWF \\
\hline $\begin{array}{l}\text { 1. CIVIL } \\
\text { CONSTRUCTION }\end{array}$ & & & & & & & 20 & & 20 & & 20 & & & & & & & & & & & & & \\
\hline 2. CRYOGENICS & 95 & 100 & 95 & 100 & 95 & 100 & 5 & 120 & 5 & 120 & 5 & 120 & & & & & & & & & & & & \\
\hline $\begin{array}{l}\text { 3. POWER SUPPLY Q OPM } \\
\text { (SOFTWARE) }\end{array}$ & & & & & ' & & & $\begin{array}{l}50 \\
30\end{array}$ & & $\begin{array}{l}50 \\
30\end{array}$ & & $\begin{array}{l}50 \\
30\end{array}$ & . & & & & & & & & & & & \\
\hline $\begin{array}{l}\text { 4. CONTROLS AND DATA } \\
\text { COLLECTION } \\
\text { (SOFTWARE) }\end{array}$ & & & & & & & & 60 & & 60 & & 60 & & & & & & & & & & & & \\
\hline 5. SAFETY & & & & & & & & & & & & & & & & & & & & & & & & \\
\hline $\begin{array}{l}\text { 6. INSTALLATION } \\
\text { - MECHANICAL } \\
\text { ELECTRICAL } \\
\end{array}$ & & & & & & & 25 & $\begin{array}{l}40 \\
20\end{array}$ & 25 & $\begin{array}{l}40 \\
20\end{array}$ & 50 & $\begin{array}{l}40 \\
20\end{array}$ & & & & & & & & & & & & \\
\hline 7. TEST COORDINATION & & & & & & & & 30 & & 30 & & 30 & & & & & & & & & & & & \\
\hline 8 miscellaneoús & & & & & & & 20 & & 20 & & 20 & & & & & & & & & & & & & \\
\hline 9 CRYOGENIC FLUIDS & & & & & & & 3 & & 1 & & 1 & & & & & & & & & & & & & \\
\hline 10. POWER & & & & & & & & & & & & & & & & & & & & & & & & \\
\hline II TOTALS & 95 & 100 & 95 & 100 & 95 & 100 & 73 & 350 & 71 & 350 & 96 & 350 & & & & & & & & & & & & \\
\hline 12 TOTALS & \multicolumn{2}{|c|}{195} & \multicolumn{2}{|c|}{195} & \multicolumn{2}{|c|}{195} & \multicolumn{2}{|c|}{423} & \multicolumn{2}{|c|}{421} & \multicolumn{2}{|c|}{446} & & & & & & & & & & & & \\
\hline & & & & & & & & DC & T) & & B A & & & & & & ANO TO & TALS & & 18 & & 16 & & 41 \\
\hline
\end{tabular}




$$
v-4
$$

OPERATING COST (K\$) - TOTAL

\begin{tabular}{|c|c|c|c|c|c|c|c|c|c|c|c|c|c|c|c|c|c|c|c|c|c|c|c|c|}
\hline & \multicolumn{6}{|c|}{ FY'85 } & \multicolumn{6}{|c|}{ FY ' 86} & \multicolumn{6}{|c|}{ FY' 87} & \multicolumn{6}{|c|}{ FY'88 } \\
\hline & \multicolumn{2}{|c|}{ TYPEA } & \multicolumn{2}{|c|}{ TYPE } & \multicolumn{2}{|c|}{ TYPE C } & \multicolumn{2}{|c|}{ TYPEA } & \multicolumn{2}{|c|}{ TYPE B } & \multicolumn{2}{|c|}{ TYPEC } & \multicolumn{2}{|c|}{ TYPEA } & \multicolumn{2}{|c|}{ TYPE B } & \multicolumn{2}{|c|}{ TYPEC } & \multicolumn{2}{|c|}{ TYPEA } & \multicolumn{2}{|c|}{ TYPE 8} & \multicolumn{2}{|c|}{ TYPE C } \\
\hline & MBS & SWF & M \& S & SWF & Mas & SWF & Mas & SWF & Mas & SWF & MBS & SWF & MQS & SWF & M8S & SWF & mas & $5 W F$ & Mas & SWF & MQS & $5 W=$ & Mas & SWF \\
\hline \multicolumn{25}{|l|}{$\begin{array}{l}\text { 1. CIVIL } \\
\text { CONSTRUCTION }\end{array}$} \\
\hline 2. CRYOGENICS & . & & & & & & 50 & 256 & 50 & 256 & 50 & 256 & 135 & 590 & 135 & 670 & 135 & 670 & 120 & 535 & 100 & 480 & 100 & 480 \\
\hline $\begin{array}{l}\text { 3. POWER SUPPLY Q OP } \\
\end{array}$ & & & & & & & 20 & 120 & 20 & 120 & 20 & 120 & .60 & 280 & 60 & 280 & 60 & 280 & 50 & 210 & 35 & 140 & 35 & 140 \\
\hline $\begin{array}{l}\text { 4. CONTROLSAND DATA } \\
\ldots \text { COLLECTION }\end{array}$ & & & & & & & 2 & 58 & 2 & 58 & 2 & 58 & 10 & 100 & 10 & 100 & 10 & 100 & 8 & 80 & 6 & 60 & 6 & 60 \\
\hline \multicolumn{25}{|l|}{ 5. SAFETY } \\
\hline \multicolumn{25}{|l|}{$\begin{array}{l}\text { 6. INSTALLATION } \\
\text { - MECHANICAL } \\
\text { ELECTRICAL }\end{array}$} \\
\hline 7. TEST COORDINATION & & 12 & & 12 & & 12 & 2 & 90 & 2 & 90 & 2 & 90 & 5 & 240 & 5 & 240 & 5 & 240 & 5 & 180 & 5 & 120 & 5 & 120 \\
\hline \multicolumn{25}{|l|}{ 8. MISCELLANEJUS } \\
\hline 9. CRYOCENIC FLUIDS & & & & & & & 115 & & 60 & & 50 & & 346 & & 202 & & 210 & & 173 & & 60 & & 50 & \\
\hline 10. POWE? & & & & & & & 200 & & 165 & & 160 & & 730 & & 665 & & 650 & & 370 & & 165 & & 160 & \\
\hline II. TOTALSS & & 12 & & 12 & & 12 & 389 & 524 & 299 & 524 & 284 & 524 & 1286 & 1210 & .1077 & 1290 & 1070 & 1290 & 726 & 1005 & 371 & 800 & 356 & 800 \\
\hline 12. TOTALS & \multicolumn{2}{|c|}{12} & \multicolumn{2}{|c|}{12} & \multicolumn{2}{|c|}{12} & \multicolumn{2}{|c|}{913} & \multicolumn{2}{|c|}{823} & \multicolumn{2}{|c|}{808} & \multicolumn{2}{|c|}{2496} & \multicolumn{2}{|c|}{2367} & \multicolumn{2}{|c|}{2360} & & 31 & & 171 & 115 & \\
\hline & - & & & & & & & 0 & 8 & & & & & & & & ND TC & ALS & & 152 & & 373 & 433 & \\
\hline
\end{tabular}




\begin{tabular}{|c|c|c|c|c|c|c|c|c|c|c|c|c|c|c|c|c|c|c|c|c|c|c|c|c|}
\hline & \multicolumn{6}{|c|}{ FY' 85} & \multicolumn{6}{|c|}{ FY ' 86} & \multicolumn{6}{|c|}{ FY '87 } & \multicolumn{6}{|c|}{ FY 88} \\
\hline$\cdots$ & \multicolumn{2}{|c|}{ TYPE A } & \multicolumn{2}{|c|}{ TYPE B } & \multicolumn{2}{|c|}{ TYPE C } & \multicolumn{2}{|c|}{ TYPE A } & \multicolumn{2}{|c|}{ TYPE $B$} & \multicolumn{2}{|c|}{ TYPE C } & \multicolumn{2}{|c|}{ TYPE A } & \multicolumn{2}{|c|}{ TYPE B } & \multicolumn{2}{|c|}{ TYPE C } & \multicolumn{2}{|c|}{ TYPEA } & \multicolumn{2}{|c|}{ TYPE B } & \multicolumn{2}{|c|}{ TYPE C } \\
\hline & MBS & SWF & Mas & SWF & Mas & SWF & MAS & SWF & M85 & SWF & Mas & SWF & Mas & sWr & Mas & SWF & Mas & SWF & MBS & SWF & $M A S$ & $s W F$ & in BS & SWF \\
\hline 2. CRYOGENICS & & & & & & & 22 & 120 & 22 & 120 & 22 & 120 & 100 & 430 & 100 & 430 & 100 & 430 & 72 & 320 & 45 & 210 & 45 & 210 \\
\hline $\begin{array}{l}\text { 3. POWER SUPPLY } \mathrm{Q} \text { OPM } \\
\text { (SOFTWARE) }\end{array}$ & & & & & & & 30 & $\begin{array}{r}130 \\
90\end{array}$ & 30 & $\begin{array}{r}130 \\
90\end{array}$ & 60 & $\begin{array}{r}130 \\
90\end{array}$ & 70 & $\begin{array}{r}170 \\
90\end{array}$ & 70 & $\begin{array}{r}170 \\
90\end{array}$ & 140 & $\begin{array}{r}170 \\
90\end{array}$ & 60 & $\begin{array}{r}100 \\
60\end{array}$ & 50 & $\begin{array}{r}100 \\
60\end{array}$ & 100 & $\begin{array}{r}100 \\
60\end{array}$ \\
\hline $\begin{array}{l}\text { 4. CONTROLS AND DATA } \\
\text { COLLECTION } \\
\text { (SOFTWARE) }\end{array}$ & & & & & & & & & & & & & & 30 & & 30 & & 30 & & 15 & & & & \\
\hline 5. SAFETY & & & & & & & & & & & & $\cdot$ & & & & & & & & & & & & \\
\hline $\begin{array}{l}\text { 6. INSTALLATION } \\
\text { - MECHANICAL } \\
\therefore \text { ELECTRICAL }\end{array}$ & & & & & & & & $\begin{array}{l}80 \\
40\end{array}$ & & $\begin{array}{l}80 \\
40\end{array}$ & & $\begin{array}{l}80 \\
40\end{array}$ & & $\begin{array}{l}80 \\
40\end{array}$ & & $\begin{array}{l}80 \\
40\end{array}$ & & $\begin{array}{l}80 \\
40\end{array}$ & & $\begin{array}{l}80 \\
40\end{array}$ & & $\begin{array}{l}80 \\
40\end{array}$ & & $\begin{array}{l}80 \\
40\end{array}$ \\
\hline 7. TEST COORDINATION & & & & & & & & 180 & & 180 & & 180 & & 210 & & 210 & & 210 & & 195 & & 180 & & 180 \\
\hline 8. MISCELLANEOUS & & & & & & & 25 & & 25 & & 25 & & 90 & & 90 & & 90 & & 70 & & 50 & & 50 & \\
\hline 9. CAYOGENIC FLUIDS & & & & & & & 35 & & 35 & & 35 & & 825 & & 375 & & 330 & & 120 & & 70 & & 70 & \\
\hline 10. POWER & & & & & & & 75 & & 75 & & 75 & & 625 & & 475 & & 475 & & 238 & & 150 & & 150 & \\
\hline II. TOTALS & & & & & & & 187 & 640 & 187 & 640 & 217 & 640 & 1710 & 1050 & 1110 & 1050 & 1135 & 1050 & $36 n$ & 810 & 365 & 670 & 415 & 670 \\
\hline 12 TOTALS & & & & & & & 82 & $?$ & 82 & 7 & 85 & & 276 & & & 160 & & 185 & 13 & 70 & & 035 & & 1085 \\
\hline & & & & & & & & & & & & & & & & & AND $T$ & TALS & & 4957 & & 022 & & 4127 \\
\hline
\end{tabular}


$v-5$

OPERATING COST ( $K \$)$-LONG STRLNG,

\begin{tabular}{|c|c|c|c|c|c|c|c|c|c|c|c|c|c|c|c|c|c|c|c|c|c|c|c|c|}
\hline & \multicolumn{6}{|c|}{ FY' 85} & \multicolumn{6}{|c|}{ FY' 86} & \multicolumn{6}{|c|}{ FY '87 } & \multicolumn{6}{|c|}{ FY'88 } \\
\hline & \multicolumn{2}{|c|}{ TYPEA } & \multicolumn{2}{|c|}{ TYPE B } & \multicolumn{2}{|c|}{ TYPE C } & \multicolumn{2}{|c|}{ TYPEA } & \multicolumn{2}{|c|}{ TYPE B } & \multicolumn{2}{|c|}{ TYPE C } & \multicolumn{2}{|c|}{ TYPE A } & \multicolumn{2}{|c|}{ TYPE $\mathrm{B}$} & \multicolumn{2}{|c|}{ TYPE C } & \multicolumn{2}{|c|}{ TYPEA } & \multicolumn{2}{|c|}{ TYPE $B$} & \multicolumn{2}{|c|}{ TYPE $C$} \\
\hline & Mas & SWF & MBS & SWF & MAS & SWF & MAS & SWF & MBS & $S W F$ & mas & SWF & Mas & SWF & Mas & SWF & MaS & SWF & Mas & SWF & Mas & SWF & Mas & SWF \\
\hline \multicolumn{25}{|l|}{$\begin{array}{l}\text { 1. CIVIL } \\
\text { CONSTRUCTION }\end{array}$} \\
\hline 2. CRYOGENICS & & & & & & & 50 & 256 & 50 & 256 & 50 & 256 & 100 & 480 & 100 & 480 & $10 \mathrm{C}$ & 480 & 100 & 480 & 100 & 480 & 100 & 480 \\
\hline 3. POWER SUPPLY B QP & & & & & & & 20 & 120 & 20 & 120 & 20 & 120 & -35 & 140 & 35 & 140 & 35 & 140 & 35 & 140 & 35 & 140 & 35 & 140 \\
\hline $\begin{array}{l}\text { 4. CONTROLS AND DATA } \\
\therefore \text { COLLECTION }\end{array}$ & - & 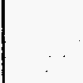 & & & & & 2 & 58 & 2 & 58 & 2 & 58 & 6 & 60 & 6 & 60 & 6 & 60 & 6 & 60 & 6 & 60 & 6 & 60 \\
\hline \multicolumn{25}{|l|}{ 5. SAFETY } \\
\hline $\begin{array}{l}\text { 6. INSTALLATION } \\
\text { MECHANICAL } \\
\text { - ELECTRICAL }\end{array}$ & $\ldots \ldots .$. & $\cdots$ & & & & $\cdots$ & & & & & & & & & - & & 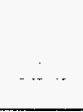 & & & & & & . & - \\
\hline 7. TEST COORDINATION & & 6 & & 6 & & 6 & 2 & 48 & 2 & 48 & 2 & 48 & 5 & 120 & 5 & 120 & 5 & 120 & 5 & 120 & 5 & 120 & 5 & 120 \\
\hline \multicolumn{25}{|l|}{ B. MISCELLANEOUS } \\
\hline 9. CRYOGENIC FLUIDS & & & & & & & 115 & & 60 & & 50 & & 230 & & 120 & & 100 & & 115 & & 60 & & 50 & \\
\hline 10. POWER & & & & & & & 200 & & 165 & & 160 & & 395 & & 330 & & 315 & & 200 & & 165 & & 160 & \\
\hline II. TOTALS & & 6 & & 6 & & 6 & 389 & 482 & 299 & 482 & 284 & 482 & 771 & 800 & 596 & 800 & 561 & 800 & 461 & 800 & 371 & 800 & 356 & 800 \\
\hline 12 TOTALS & & 6 & & 6 & & 6 & & 371 & & 81 & 76 & & 157 & & 139 & & 136 & & 12 & & 117 & & 1156 & \\
\hline & & & & & & & & & & & & & & & & & AND TO & TALS & 370 & & 335 & & 3289 & \\
\hline
\end{tabular}




\begin{tabular}{|c|c|c|c|c|c|c|c|c|c|c|c|c|c|c|c|c|c|c|c|c|c|c|c|c|}
\hline & \multicolumn{6}{|c|}{ FY '85 } & \multicolumn{6}{|c|}{ FY '86 } & \multicolumn{6}{|c|}{ FY' 87} & \multicolumn{6}{|c|}{ FY ' 88} \\
\hline & \multicolumn{2}{|c|}{ TYPE A } & \multicolumn{2}{|c|}{ TrPE $\mathrm{\theta}$} & \multicolumn{2}{|c|}{ TYPE C } & \multicolumn{2}{|c|}{ TYPEA } & \multicolumn{2}{|c|}{ TYPE B } & \multicolumn{2}{|c|}{ TYPE C } & \multicolumn{2}{|c|}{ TYPE A } & \multicolumn{2}{|c|}{ TYPE $\mathbf{B}$} & \multicolumn{2}{|c|}{ TYPE C } & \multicolumn{2}{|c|}{ TYPE A } & \multicolumn{2}{|c|}{ TYPE $B$} & \multicolumn{2}{|c|}{ TYPE $C$} \\
\hline & mas & SWF & Mas & SWF & MaS & swF & MBS & SWF & MAS & SWF & Mas & SWF & MAS & SWF & MQS & SWF & MaS & $\overline{\text { SWF }}$ & Mas & SWF & Mas & SWF & Mas & sWF \\
\hline \multicolumn{25}{|l|}{$\begin{array}{l}\text { 1. CIVIL } \\
\text { CONSTRUCTION }\end{array}$} \\
\hline 2. CRYOGENICS & & & & & & & 22 & 120 & 22 & 120 & 22 & 120 & 45 & 210 & 45 & 210 & 45 & 210 & 45 & 210 & 45 & 210 & 45 & 210 \\
\hline $\begin{array}{l}\text { 3. POWER SUPPLY Q QPM } \\
\text { (SOFTWARE) }\end{array}$ & & & & & I & & 25 & $\begin{array}{r}130 \\
90\end{array}$ & 25 & $\begin{array}{r}130 \\
90\end{array}$ & 50 & $\begin{array}{r}130 \\
90\end{array}$ & 50 & $\begin{array}{r}170 \\
90\end{array}$ & 50 & $\begin{array}{r}170 \\
90\end{array}$ & 100 & $\begin{array}{r}170 \\
90\end{array}$ & 50 & $\begin{array}{r}100 \\
60\end{array}$ & 50 & $\begin{array}{r}100 \\
60\end{array}$ & 100 & $\begin{array}{r}100 \\
60\end{array}$ \\
\hline \multicolumn{25}{|l|}{$\begin{array}{l}\text { 4. CONTROLS AND DATA } \\
\text { - COLLECTION } \\
\text { (SOFTWARE) }\end{array}$} \\
\hline \multicolumn{25}{|l|}{ 5. SAFETY } \\
\hline $\begin{array}{l}\text { 6. INSTALLATION } \\
\text { MECHANICAL } \\
\text { ELECTRICAL }\end{array}$ & & & & & & & & $\begin{array}{l}80 \\
40 \\
\end{array}$ & & $\begin{array}{l}80 \\
40 \\
\end{array}$ & & $\begin{array}{l}80 \\
40\end{array}$ & & $\begin{array}{l}80 \\
40\end{array}$ & & $\begin{array}{l}80 \\
40\end{array}$ & & $\begin{array}{l}80 \\
40\end{array}$ & & $\begin{array}{l}80 \\
40\end{array}$ & & $\begin{array}{l}80 \\
40\end{array}$ & & $\begin{array}{l}80 \\
40\end{array}$ \\
\hline 7. TEST COORDINATION & & & & & & & & 180 & & 180 & & 180 & & 180 & & 180 & & 180 & & 180 & & 180 & & 180 \\
\hline B. MISCELLANEOUS & & & & & & & 25 & & 25 & & 25 & & 50 & & 50 & & 50 & & 50 & & 50 & & 50 & \\
\hline 9. CRYOGENIC FLUIDS & & & & & & & 35 & & 35 & & 35 & & 600 & & 240 & & 170 & & 70 & & 70 & & 70 & \\
\hline 10. POWER & & & & & & & 75 & & 75 & & 75 & & 450 & & 300 & & 300 & & 150 & & 150 & & 150 & \\
\hline 11. TOTALS & & & & & & & 182 & 640 & 182 & 640 & 207 & 640 & 1195 & 770 & 685 & 770 & 665 & 770 & 365 & 670 & 365 & 670 & 415 & 670 \\
\hline 12 TOTALS & & & & & & & \multicolumn{2}{|c|}{$822^{\circ}$} & \multicolumn{2}{|c|}{822} & \multicolumn{2}{|c|}{847} & \multicolumn{2}{|c|}{1965} & \multicolumn{2}{|c|}{1455} & \multicolumn{2}{|c|}{1435} & \multicolumn{2}{|c|}{1035} & \multicolumn{2}{|c|}{1035} & \multicolumn{2}{|c|}{1085} \\
\hline
\end{tabular}

NOTE: The increased Cryo fluids and power costs in FY87

(vs FY86 and 88) are required for the full string quenches

(5 each aperture), the half-cell quenches $(150)$ and the 
$\mathrm{v}-6$

Brookhaven

OPERATING COST (K.\$)-SHORT STRING

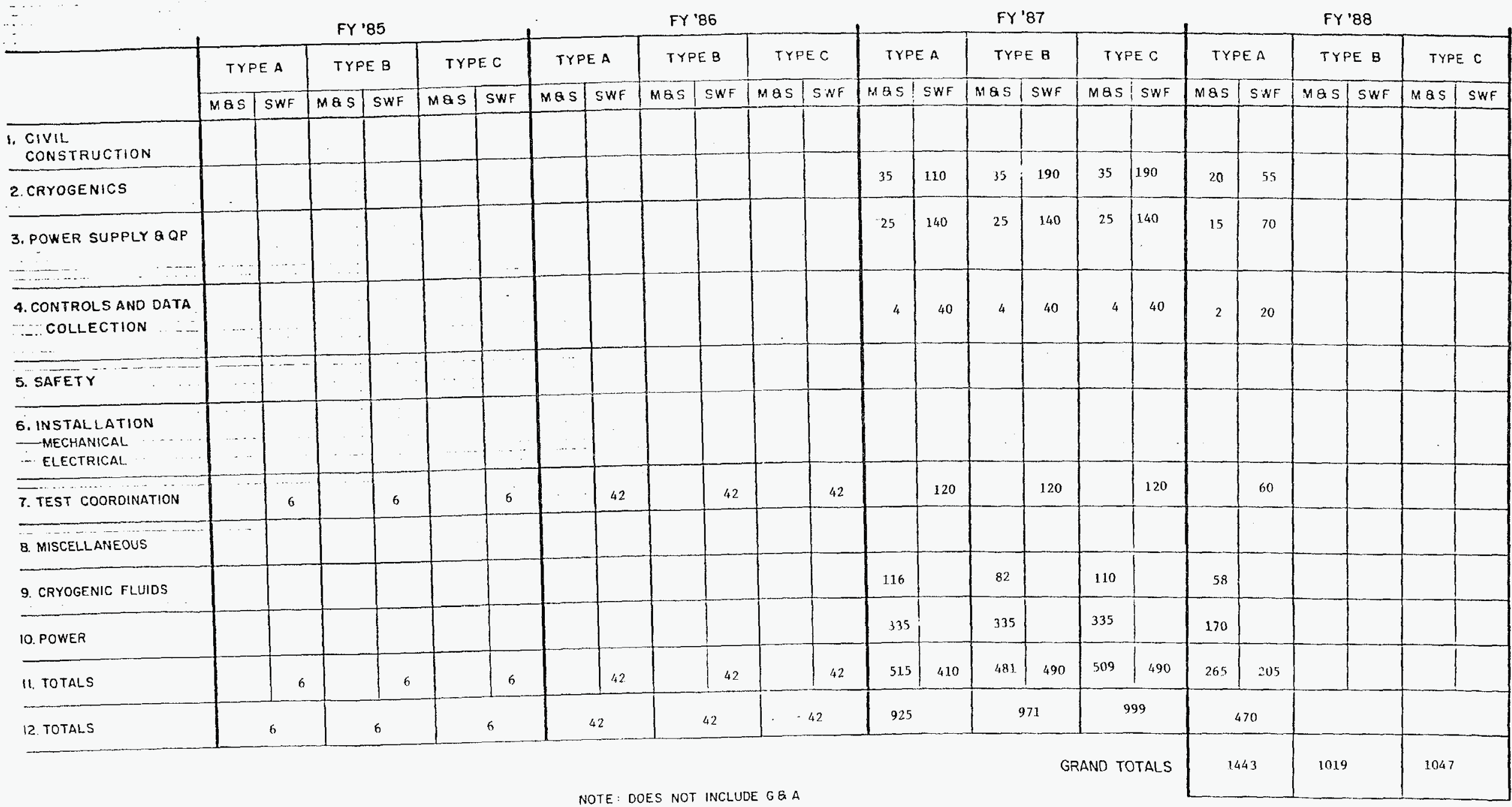




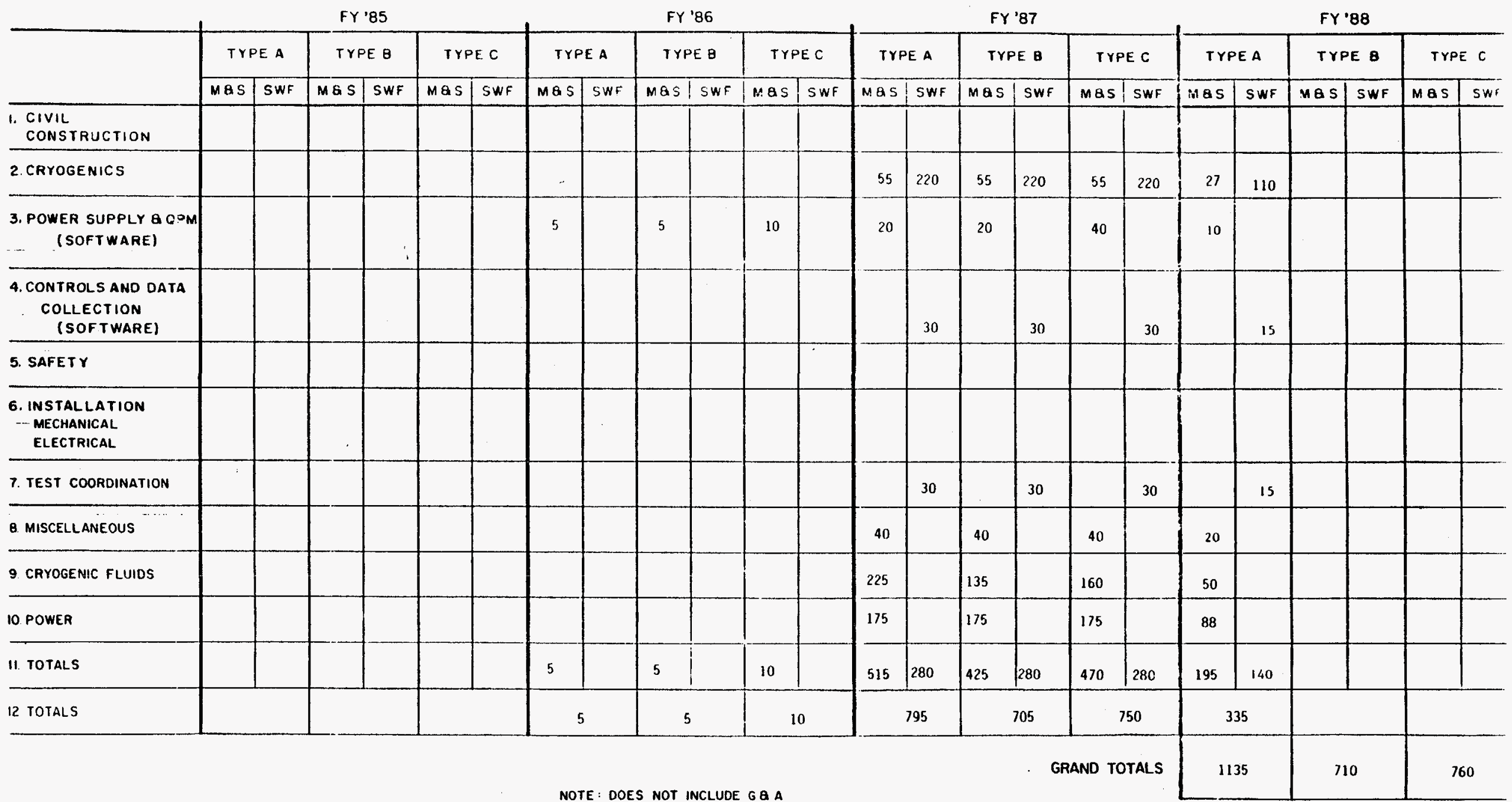

NOTE : DOES NOT INCLUDE GQ A 
Brookhoven

V - 7

MANPOWER (MAN YEARS) - TOTAL

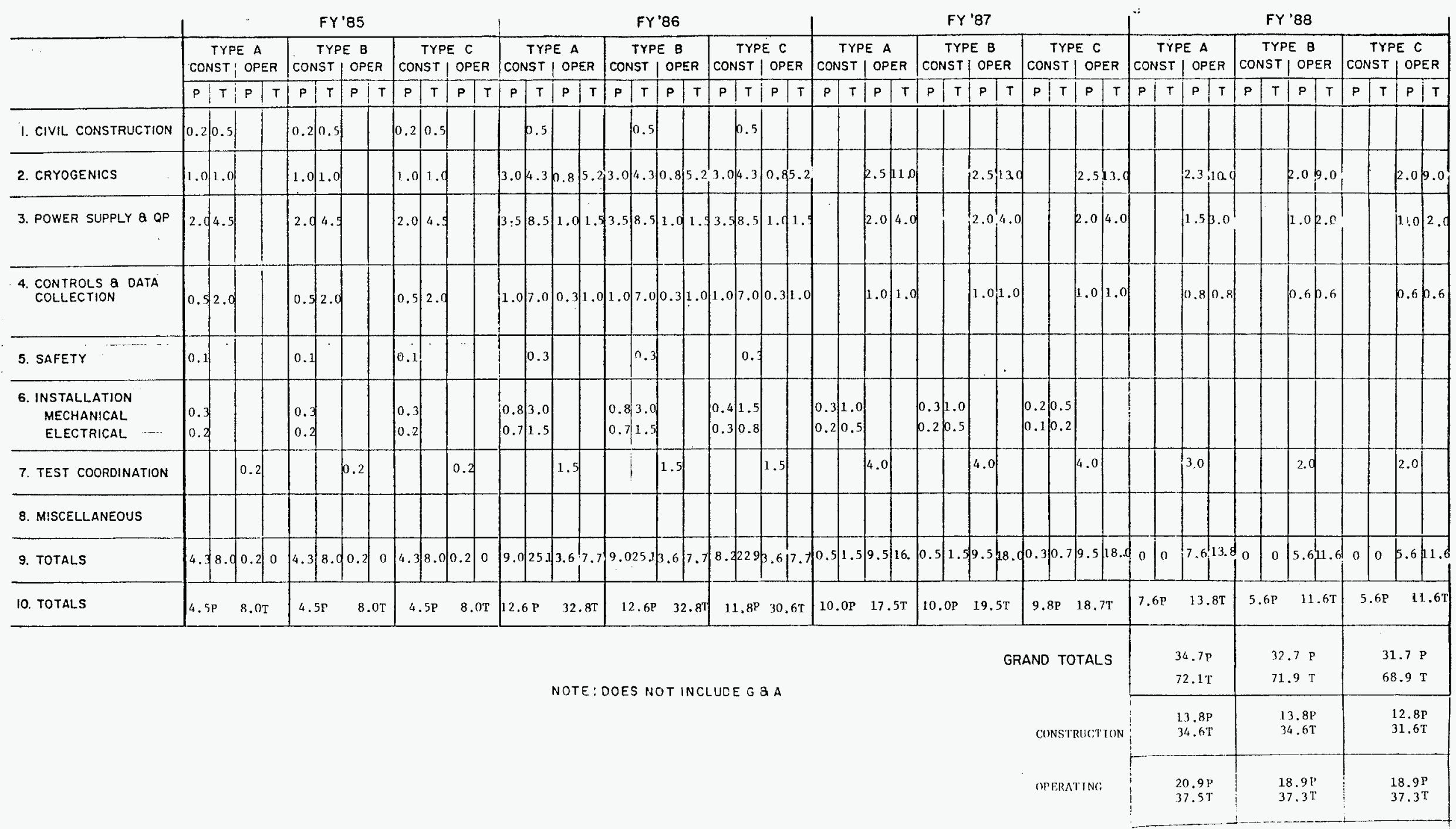




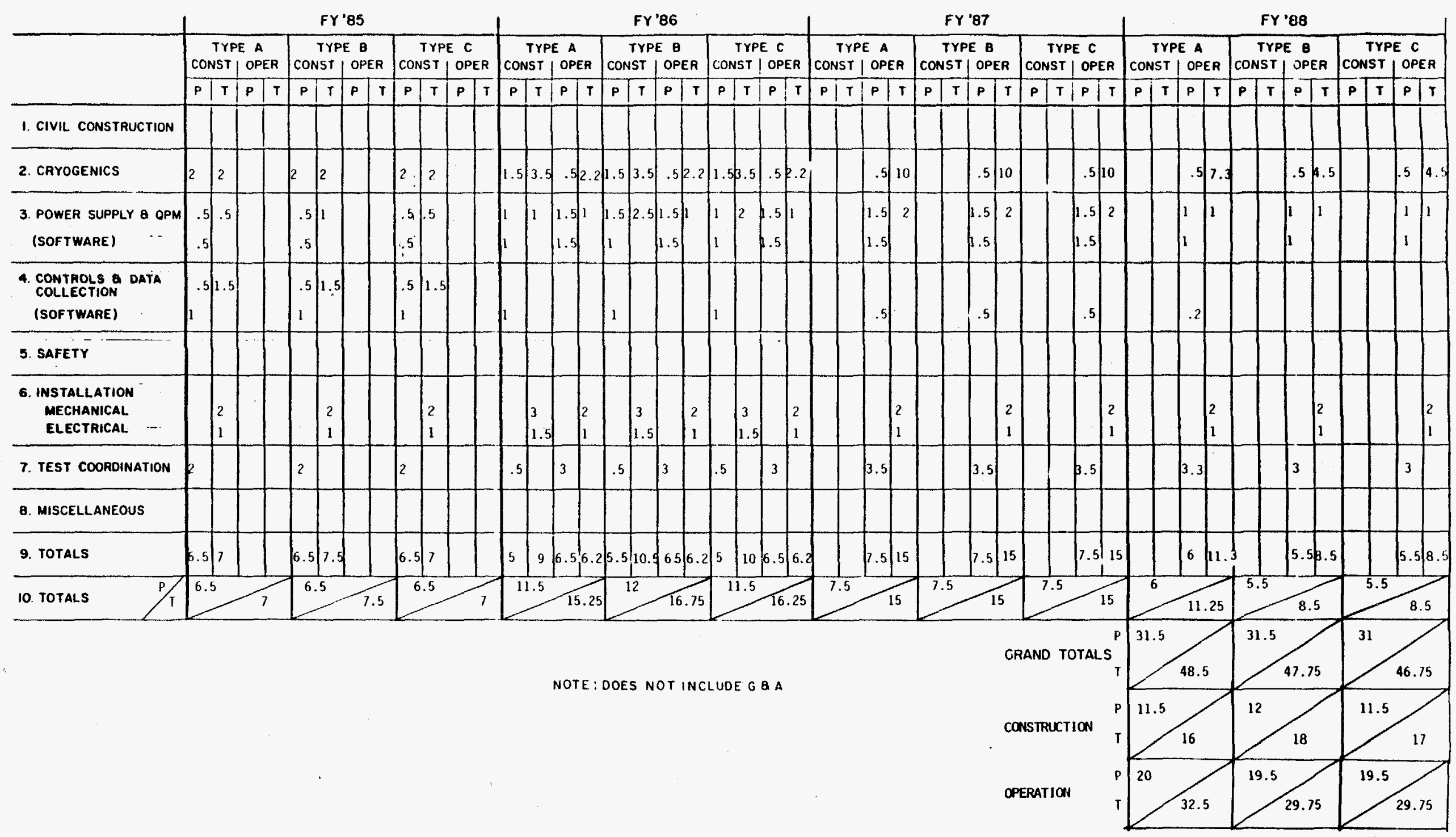




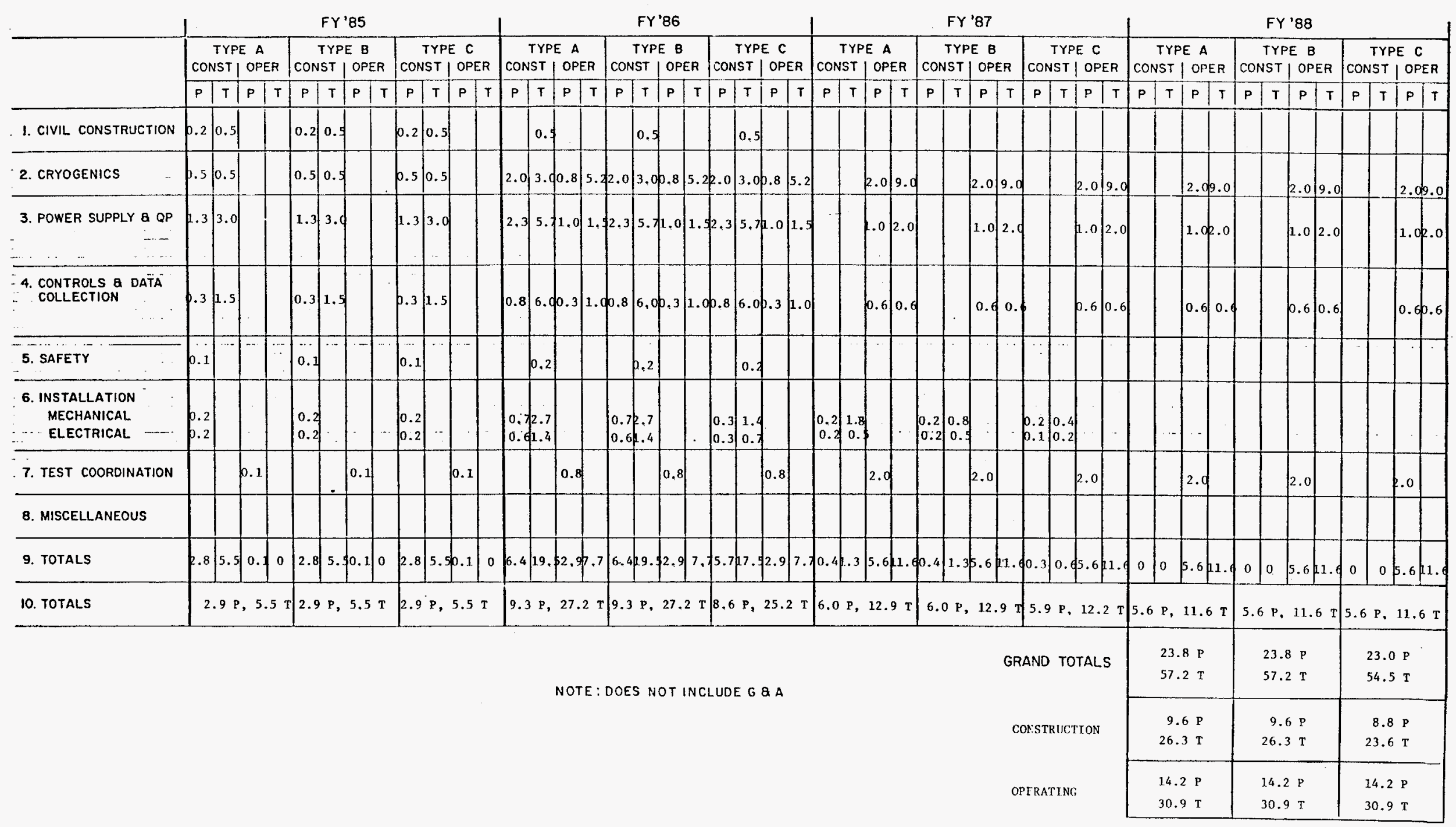




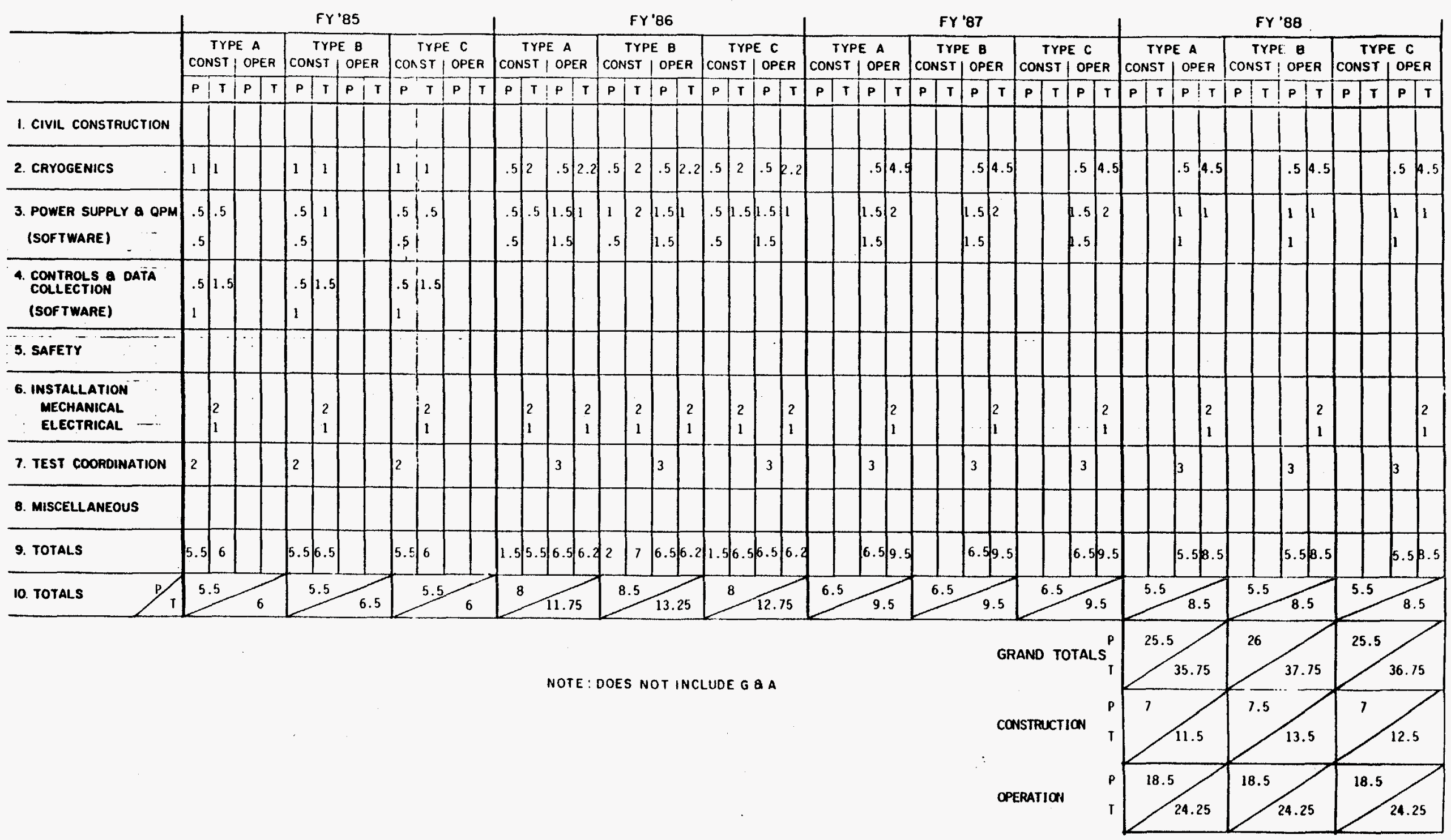




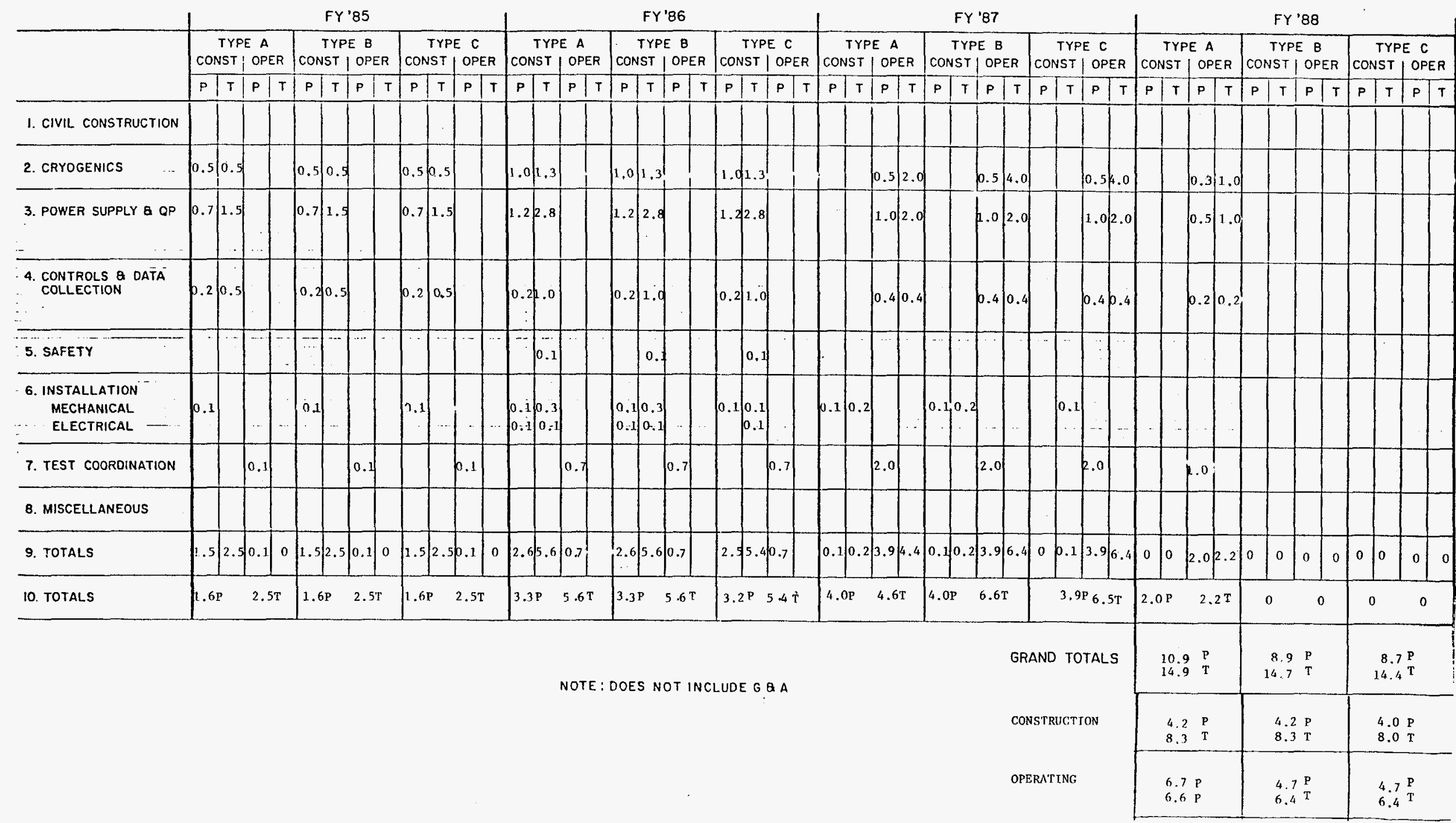




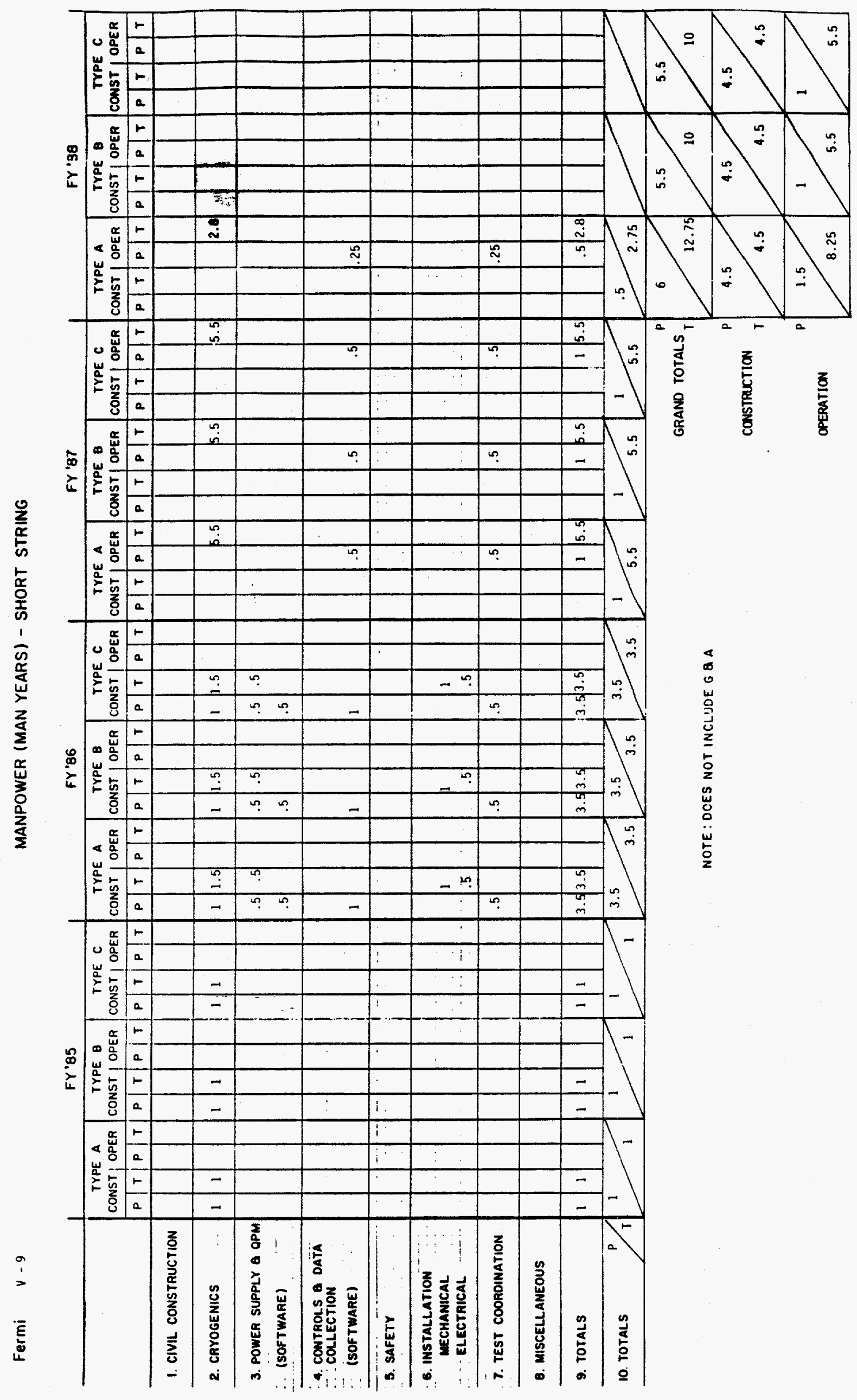




\section{Civil Construction}

Long String Test

Tunne1

Type A $\frac{\text { Type B }}{115} \quad$ Type C

Control Room

115

115

115

offices

10

10

10

Refrigerator Building

Technician Work Area

Total Cost (KS)

27

27

27

Short String Test

Tunnel
Control Room
Offices
Refrigerator Building
Technician Works Area
Total Cost (K\$)

$\frac{\text { Type A }}{-}$

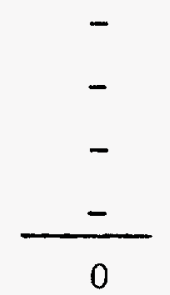

Type B
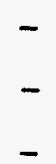

-

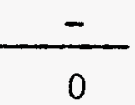

Type C
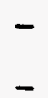
TABLE $\quad V-10$

COST OF CIVIL CONSTRUCTION

Long String: Phase I ( $\$ \mathrm{~K})$ (Lab. Fermi)

Tunne1

Control Room

Offices

Refrigerator Building

Technican Works Area

TOTAL COST

\begin{tabular}{cccc} 
Type A & & Type B & Type C \\
117 & & 117 & 117 \\
- & - & - \\
- & - & - \\
17 & 17 & 17 \\
32 & & 32 & 32 \\
\hline 166 & & 166 & 166
\end{tabular}

Tunnel

Control Room

Offices

Refrigerator Building

Technician Works Area

TOTAL COST

TOTAL COST

Long String: Phase II (\$K)

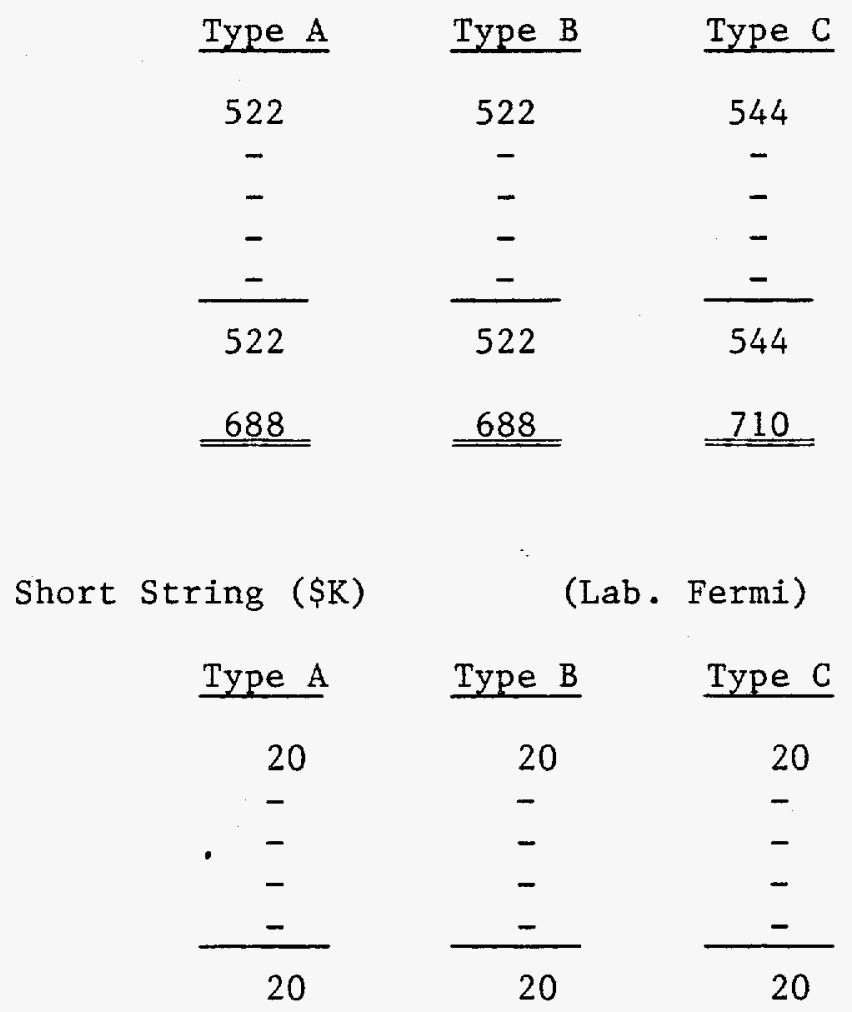

\begin{tabular}{|c|c|c|}
\hline Type A & Type B & Type C \\
\hline 522 & 522 & 544 \\
\hline- & - & - \\
\hline - & - & - \\
\hline - & - & - \\
\hline - & - & - \\
\hline 522 & 522 & 544 \\
\hline 688 & 688 & 710 \\
\hline Short String ( $\$ \mathrm{~K})$ & (Lab. & Fermi) \\
\hline Type A & Type B & Type C \\
\hline 20 & 20 & 20 \\
\hline- & - & - \\
\hline . $\quad-$ & - & - \\
\hline - & - & - \\
\hline 20 & 20 & 20 \\
\hline
\end{tabular}

Tunne1

Control Room

Offices

Refrigerator Building

Technician Works Area

TOTAL COST 
TABLE V-11

Brookhaven

CRYOGENIC CAPACITIES - LONG STRING

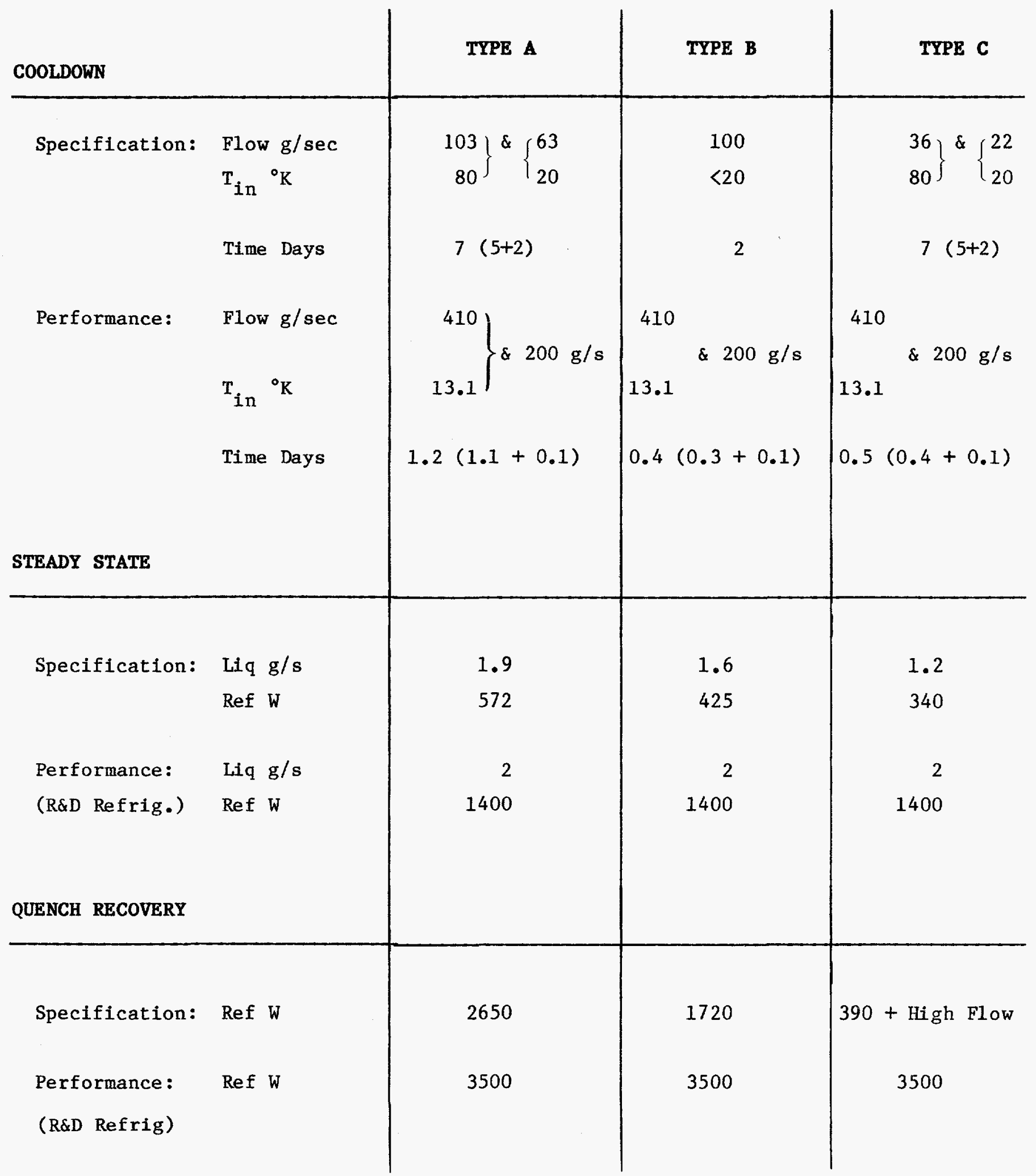


TABLE V - 11 (FNAL)
Rev. $10-2-84$

Fermi

CRYOGENIC CAPACITIES - LONG STRING

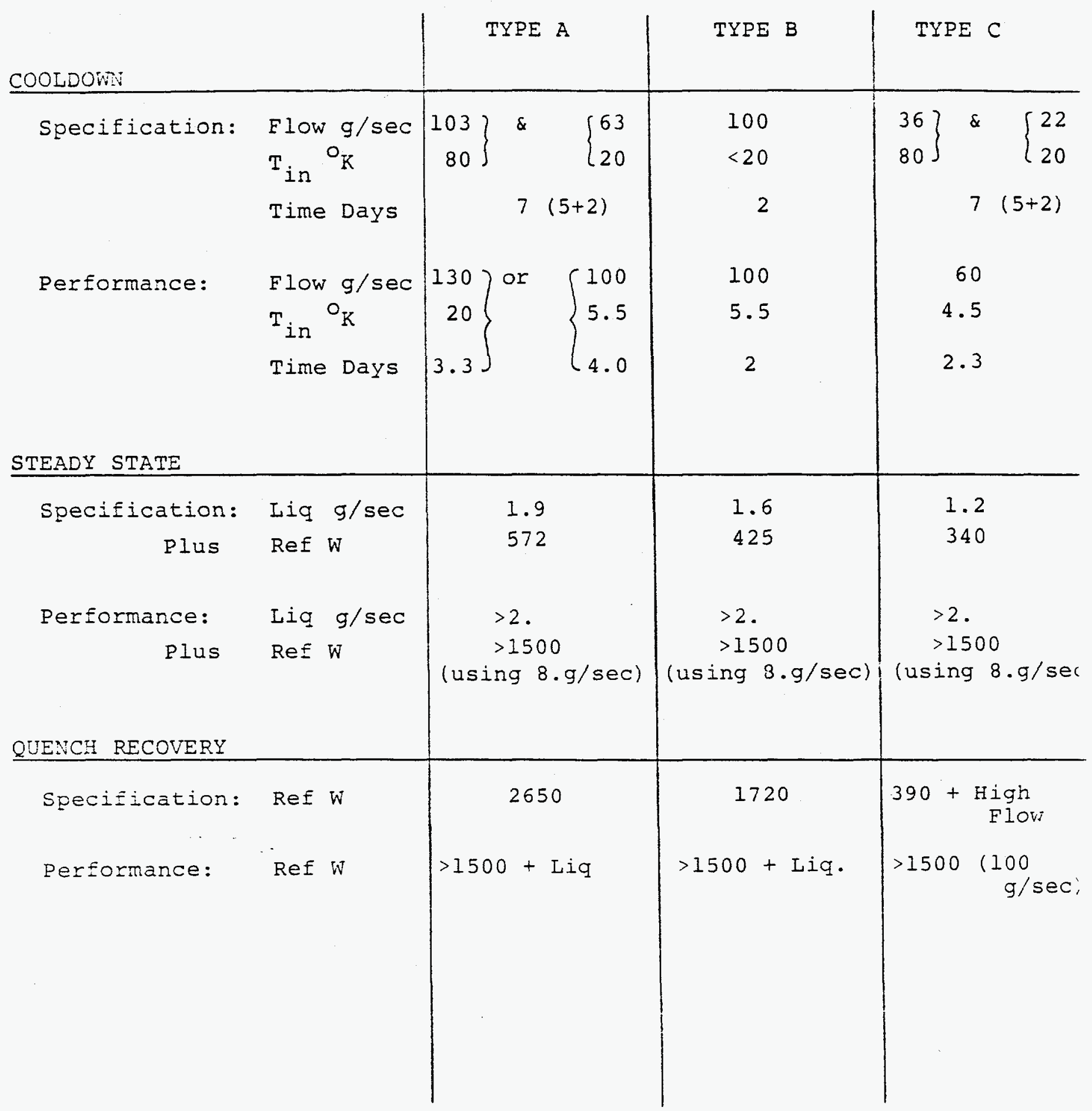


TABLE $\mathbf{V}-12$

\section{Brookhaven}

CRYOGENIC EQUIPMENT NEEDED - LONG STRING

\begin{tabular}{|c|c|c|c|c|}
\hline COMPONENT & TYPE A & TYPE B & & TYPE $\mathrm{C}$ \\
\hline Helium - Air HX & $15 \mathrm{~K}$ & $15 \mathrm{~K}$ & $\$$ & $15 \mathrm{~K}$ \\
\hline He - 1st Charge & 30 & 15 & & 6 \\
\hline $\mathrm{LN}_{2}$ Pump & 2 & 2 & & 2 \\
\hline $\mathrm{LN}_{2}$ Valves \& Piping & 10 & 10 & & 10 \\
\hline He V.J. Transfer Lines & 20 & 20 & & 20 \\
\hline Warm Return & 68 & 68 & & 68 \\
\hline TOTAL & $145 \mathrm{~K}$ & $130 \mathrm{~K}$ & $\$$ & $121 \mathrm{~K}$ \\
\hline
\end{tabular}


Fermi

CRYOGENIC EQUIPMENT NEEDED - LONG STRING

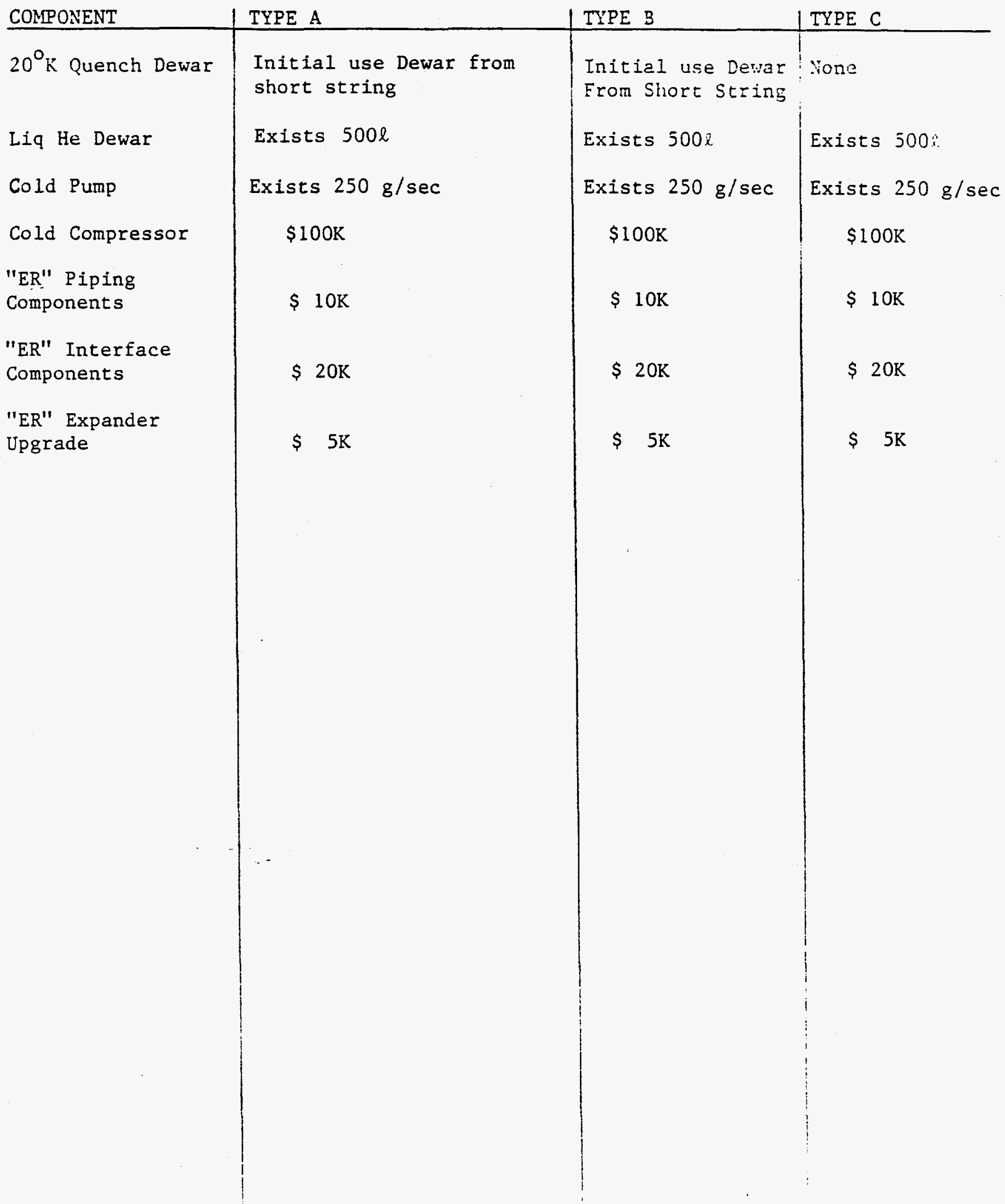


TABLE $\quad \nabla-13$

Brookhaven

CRYOGENIC CAPACITIES - SHORT STRING

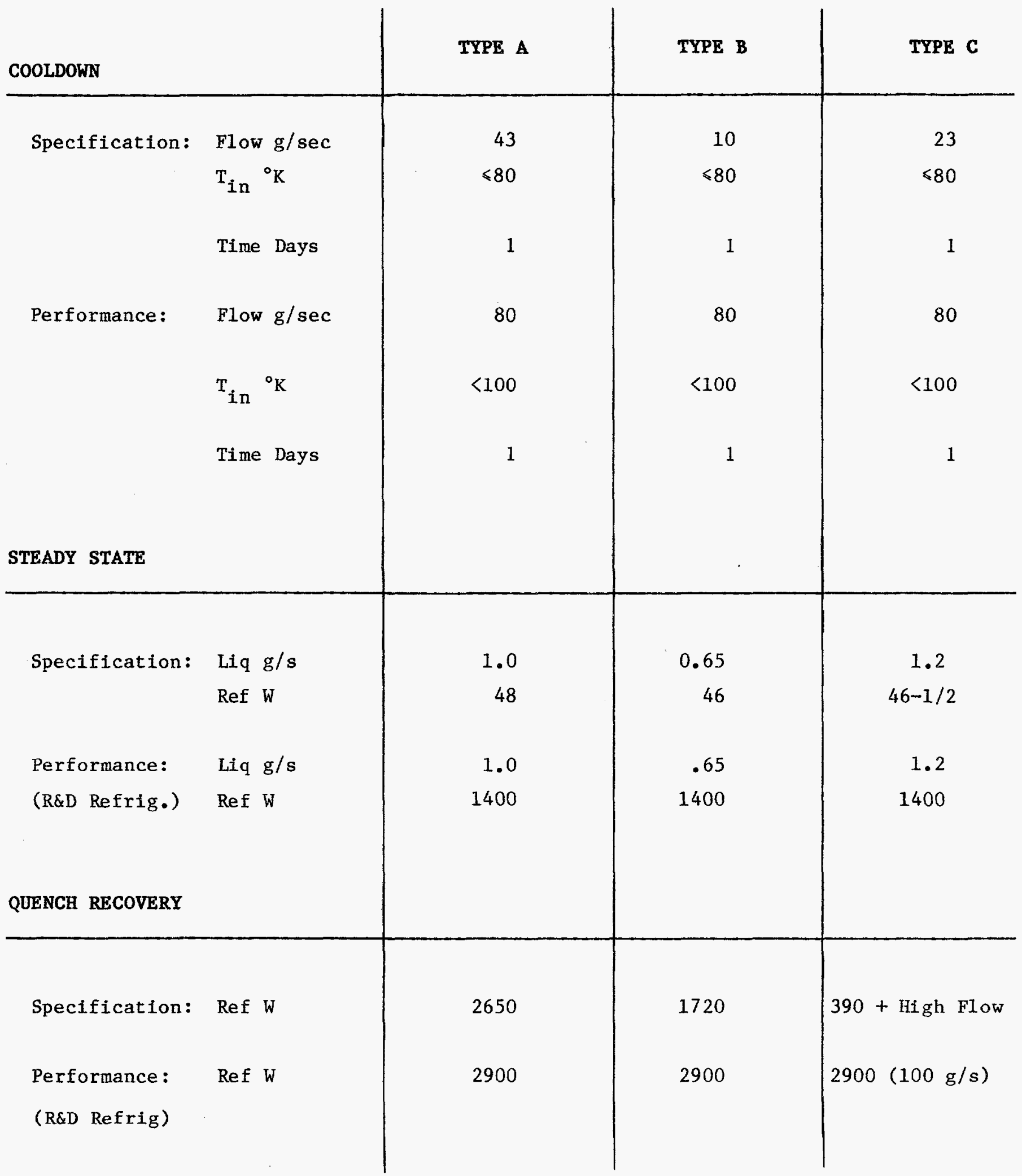




$$
\text { TABLE V - } 13 \text { (FNAL) Rev 10-2-84 }
$$

Fermi

CRYOGENIC CAPACITIES - SHORT STRING

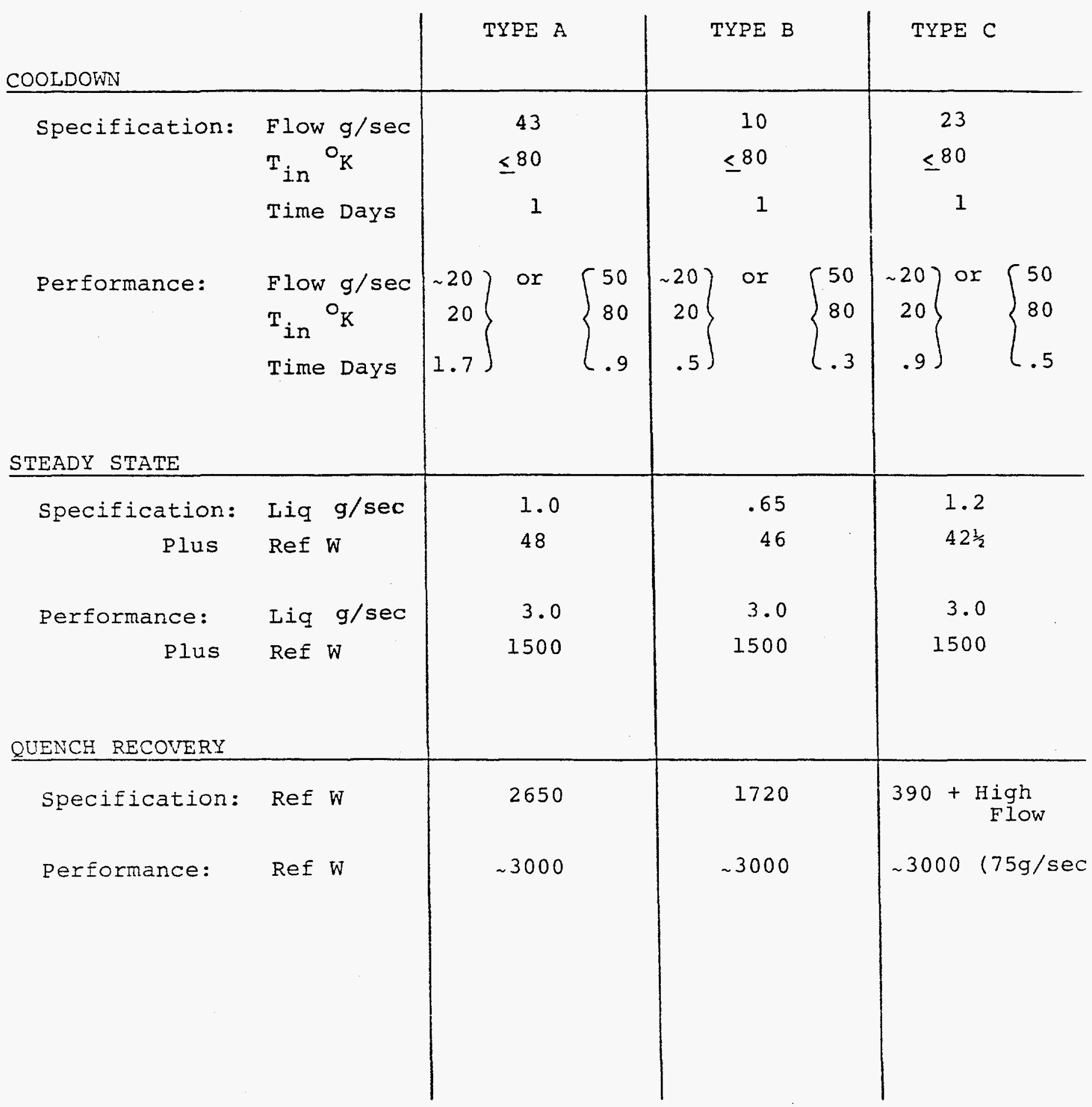


TABLE V-14

Brookhaven

CRYOGENIC EQUIPMENT NEEDED - SHORT STRING

\begin{tabular}{|c|c|c|c|c|}
\hline COMPONENT & TYPE A & TYPE B & & TYPE C \\
\hline Quench Dewar $(5000$ \&) & $100 \mathrm{~K}$ & $100 \mathrm{~K}$ & \$ & $100 \mathrm{~K}$ \\
\hline He V.J. Transfer Lines & 20 & 20 & & 20 \\
\hline He Valves & 15 & 15 & & 15 \\
\hline Warm Return & 8 & 8 & & 8 \\
\hline
\end{tabular}


TABLE V - 14 (FNAL)

Fermi

CRYOGENIC EQUIPMENT NEEDED - SHORT STRING

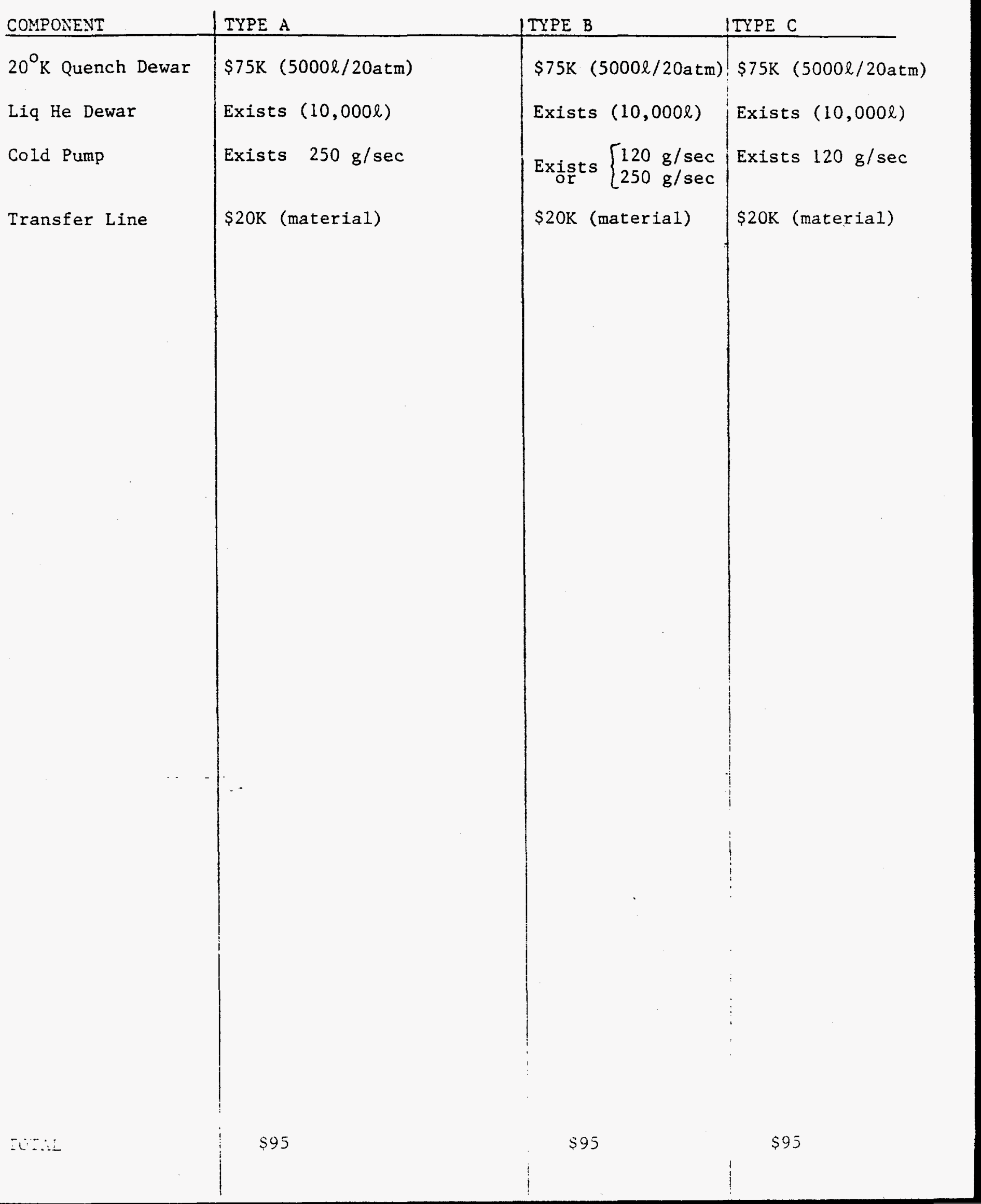


Power Supply and Quench Protection Hardware

Long String Test

Main Power Supply

Type A Type B Type C

Current (peak) (kA)

7.5

7.5

$12 / 6 / 2$

Voltage (V)

220

220

$150 / 75 / 50$

Cost (KS)

285

285

595

Trim Power Supplies

Quench Protection System

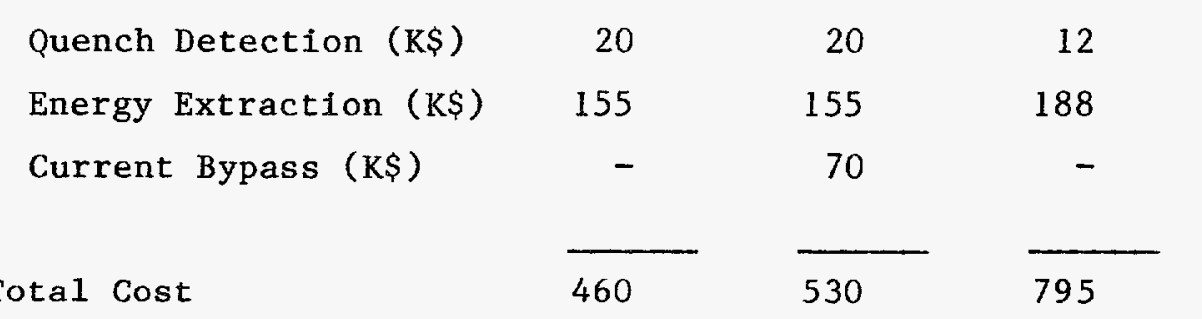


TABLE V-15 FNAL

POWER SUPPLY AND QUENCH PROTECTION EARDWARE ITEMS-LONG STRING

ITEM

Main Power Supply

Current

$\cos t$
Vol tage

M\&S

SWF

TOTAL

Trim Power Supplies

Quench Protection System

Quench Detection

$M \& S$

SWF

TOTAL

Energy Extraction

M\&S

SWF

TOTAL

Current Bypass

M\&S

SWF

TOTAL

GRAND TOTAL
IYPE A

$7 \mathrm{KA}$

$220 \mathrm{~V}$

\section{TYPE A}

TYPE B

IYPE C

$\$ 161 k$

47

$\$ 208 k$

Unknown

$\$ 67 \mathrm{k}$

95

$\$ 16 \bar{k}$

$\$ 76 \mathrm{k}$

20

$\overline{\$ 96 \mathrm{k}}$

0

0

$\overline{0}$

$\$ 466 k$
$7 \mathrm{kA}$

$220 \mathrm{~V}$

$\$ 161 k$

47

$\$ 208 k$

Unknown

$12 / 6 / 2.5 \mathrm{kA}$

$120 / 60 / 100 \mathrm{~V}$

$\$ 426 k$

63

$\$ 48 \bar{k}$

Urknown*

$\$ 67 \mathrm{k}$

95

$162 \mathrm{k}$

$\$ 172 k$

62

$\$ 234 k$

$\$ 117 k$

0

82

$\$ 199 \mathrm{k}$

$\overline{0}$

$\overline{\$ 655 k}$

- ENAl has an inventory of power supplies to use for powering trin coils. It is not likely that a complete set of the requiced supplies is on hand, so as the requirements are defined, some additional supplies will have to be purchased. 


\section{Power Supply and Quench Protection Hardware}

Short String Test

Main Power Supply

Type A Type B Type C

Current (kA)
Voltage (V)
Cost (KS)

$\begin{array}{rll}7.5 & 7.5 & 12 / 6 / 2 \\ 50 & 50 & 50 / 50 / 25 \\ 40 & 40 & 83\end{array}$

Trim Power Supplies

Quench Protection System

Quench Detection
Energy Extraction
Current Bypass

Total Cost

$\begin{array}{r}5 \\ 63 \\ - \\ \hline\end{array}$

108

$\begin{array}{r}5 \\ 63 \\ 5 \\ \hline\end{array}$

113
3

58

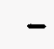

144 
TABLE V-16 FNAL

POWER SUPPLY AND QUENCH PROTECTION HARDWARE ITEMS-SHORT STRING

NONE 


\section{CONTROLS}

Long String Test

Brookahven proposes to extend the CBA process control system already in place to include Long String control and data acquisition. A host PDP 11/44 processor and redundant 11/34's are interfaced to the CBA refrigerator via a 1 megabaud data highway. Two distributed redundant $11 / 23$ 's are on hand and will be positioned along the string for magnet related process control and data acquisition. The control system supports alarm functions, long term storage, trending and three on-line configurable real time color operator consoles. The benefit derived from using this system is that the operator has the freedom to design pages of real time displays while on-line, enabling him to easily reconfigure the graphics to "zero-in" on critical or problem parameters.

Magnet quench studies require reasonably fast data acquisition techniques. Hardware can be interfaced directly to the process control distributed microcomputers in the tunnel or interfaced directly to the data highway or connected to the host via serial link. The control system host will be interfaced to the BNL On-Line Data Facility where all data analysis, plotting and simulation programs can be run, utilizing their network of VAX11780 computers and 3 gigabytes of memory.

Essentially all major hardware components necessary to perform the data acquisition and control tasks are on hand. Distributed microprocessor I/O will be reconfigured to match magnet instrumentation points, requiring additional purchased or in-house supplied hardware. Ample CAMAC hardware is available, on a loan basis, to interface to dynamic quench detection, protection and data acquisition systems. Equipment costs shown below are due mainly to reproducing interface hardware for temperature acquisition, lead flow control, and interlock and safety systems. Extention of the control system, programing and instrumentation installation and checkout will require approximately 8 man-years of effort.

Sub Total

Equi pment $(M \& S) \quad \$ 223 \mathrm{~K}$

Effort (SWF) $\quad \$ 366 \mathrm{~K}$ 
Section V.C Table 17. STF Controls (FNAL)

STF Long String Controls

Fermilab proposes to utilize the Saver controls system to support the Long String facility. A short "stub" extension of the Saver Links would be run from the E4 service building to the STF Control Portakamp. The basic control system of the Saver is a modified serial CAMAC system with the larger distributed microcomputer systems housed in multibus systems interfaced through CAMAC to the "Host" computer system. A standard protocol (GAS) has been defined and supported at Fermilab to permit direct processor to processor communications from the Host to the distributed computers. At least the following systems at the STF would initially be supported with microcomputers conforming to this protocol; vacuum, refrigeration, quench protection, main ramp generation, and higher order ramp generation. Certain other CAMAC based modules are available for correction function ramp generation and MADC readback.

The system as a whole supports slow and fast time plots (real time), logging, save-compare-restore, alarms, flexible parameter pages, high resolution color graphics, applications program development, and downloadable microcomputer development. Console control is a "standard" feature of the Saver control system; each console is identical in capability featuring interactive color monitors, precision color graphics, storage scopes and mono-chrome hardcopy, with color hardcopy services at the Main Control Room. It is proposed to supply a complete console at the STF Control Portakamp; remote console support is a routine service at Fermilab.

Essentially all the electronic components to provide these services are avai1able now from Fermilab spares; the costs are shown for replacing these spares. It is suggested in the Fermilab proposal that the SSC also fund some dedicated disk capacity. Fermilab believes that installation of the controls system would require about 1.5 years of staff effort and 1.5 years of support effort.

Sum total

(Equipment)

$\$ 82 \mathrm{~K}$

(Effort)

$\$ 150 \mathrm{~K}$

Fermilab also recommends that the SSC budget for 2 years of staff software support during each year of operation; this recommendation reflects Fermilab's style and philosophy of test development and operation. This cost is felt, however, to properly belong to the SSC R and D budget and so is not included here. 


\title{
CONTROLS
}

\author{
Short String Test
}

The Short String Test will utilize existing MAGCOOL and magnet measurement test facilities. No additional hardware for control or data acquisition need be purchased. Cryogenic control and data acquisition will be accomplished by MAGCOOL's PDP $11 / 34$ processors, programmed for magnet cooldown, test at subcooled temperature, warmup and thermal cycling. Instrumentation need only be reconnected to meet Short String Test geographic differences.

Magnet measurement will be accomplished as was done for the CBA magnet series. The host processor is a PDP 11/60 which acts as the central data facility. All necessary magnet measurement hardware is in place and operating. Information exchange between cryogenic and magnet measurement computers will be accomplished by a serial link through an "access" routine already installed in the $11 / 34$.

No hardware costs are required to implement the Short String test but about 1.5 man-years of programming time will be required to include special data acquisition, plotting and simulation programs.

Equipment $(M \& S) \quad \$ 0 \mathrm{~K}$

Effort (SWF) $\$ 84 \mathrm{~K}$ 
Tab1e V-18

STF Short String Controls (FNAL)

The assumption of this outline is that the physical location of the short test string site stretches out the west side of the Fermilab MTF area (Industrial Bldg $\|_{1}$ ) and that the existing MTF test facilities are directly connected.

With these assumptions, the four tests outlined in the test specifications (and possibly some magnetic measurements as well?) could be supported with the existing MTF measuring system, which includes scheduled upgrades replacing the present $\mathrm{PDP}-11 / 34$ with the $\mathrm{VAX} 11 / 730$ being used for TeV I magnet measurements at present. The specified tests do not call for any substantial alterations of existing single magnet test set ups or data acquisition methods. It is possible, however, that by Short String Test set up time the MTF system may be networked into Fermilab networks, making file transfers to other locations (Cluster VAXes, AD/DEVL cluster, etc.) quite routine. Therefore, no substantial alterations or additions to existing MTF facilities are required, or costed in this document for controls or data acquisition purposes.

Fermilab believes it would be imprudent not to include some contingent software effort, perhaps $1 \mathrm{MY}$ during installation and $1 / 2$ full time equivalent (or less?) during operation of the STF Short String Facility.

Cost (installation) (operation)
$1 \mathrm{MY}$

$1 / 2 \mathrm{MY} /$ year
$\$ 60 \mathrm{~K}$

$\$ 30 \mathrm{~K} /$ year 



\section{Comparisons and Conclusions}

\section{A. Introduction}

In this section the proposals of BNL and FNAL are compared to each other and to the goals of the STF program, as described in the preceding sections. An attempt has been made to make this comparison objective and quantitative. Some issues, however, are not so well defined that a purely objective assessment can be made. Also, in some cases the laboratory capabilities cannot be determined in detail relative to a requirement on the short time scale of this Task Force's existence. We have indicated the areas where there are questions and uncertainties and have attempted to point out the possible consequences.

Before presenting a comparison of the BNL and FNAL proposals, several general comments need to be made. These points are the results of general discussions within the Task Force while attempting to establish the goals of the STF program and to determine the capabilities of the two laboratories.

1. The String Test Facility (or Facilities) is recognized as a major part of the national SSC program. As such it will involve scientists from many different institutions who will actively participate in the planning and execution of the tests and the evaluation of test results. Further, it is expected that the SSC Central Design Group will play a major role in this activity by providing technical, management, and budgetary input and direction.

2. Both BNL and FNAL have exhibited commendable enthusiasm for this national effort. Considerable thought and effort have gone into their responses. This work has contributed to a better definition of the details and scope of the string tests.

3. The major facilities and capabilities of both FNAL and BNL are generally adequate to carry out the proposed STF program. There were, however, some questions raised within the Task Force as to the priority that will be given to the string test program at the two laboratories, especially during the initial two years. Potential conflicts at BNL are the development of SSC magnet type A and the proposed RHIC accelerator. Potential conflicts at FNAL are the development of the SSC magnet type B and the operation of the Tevatron program, the largest high energy physics research facility in this country. With the 
assumption of adequate financial support for this effort and the proposed SSC schedule, this Task Force concludes that either of the laboratories would be able to proceed as proposed.

4. The cost and manpower estimates presented in Section $V$ and summarized below are for reference only. Overhead, escalation, and contingency are not included. The two laboratories have attempted to carry out the estimates using the same factors, but it is possible that some variations remain.

\section{B. Facilities}

The major facilities proposed for use by BNL and FNAL will now be compared with respect to the facility requirements described in Section IV.

\section{Refrigeration System}

The System Test Facility needs refrigeration for two categories of tests:

a) Steady-state tests of a magnet string with an occasional transient, such as a quench;

b) Nonsteady-state tests such as repeated quenching and the associated warm-up and cool-down of individual magnets or a string of magnets.

Both FNAL and BNL have ample refrigeration capability for Item a) above. Item b) can be satisfied by both laboratories, but possibly not always at the rate and intensity required by the proposed test schedule.

The availability of a large liquefier at FNAL, coupled with storage for 16000 gallons of liquid helium will satisfy the requirement of Item b) above, although at times these tests may require scheduling around other activities. A cold gas pump or equivalent will be required to generate liquid helium at temperatures below $4.5 \mathrm{~K}$.

BNL will use the $R \& D$ helium refrigerator to provide cooling for the long string test except for cooldowns from ròm temperature. The R\&D refrigerator was used for the CBA Full Cell Tests and is still installed in the tunnel. The RHIC $25 \mathrm{~kW}$ refrigerator is expected to be operational before the string tests begin. It will be used to cool the string during thermal cycles, although other equipment could be used for this purpose if necessary. This refrigerator would also be available as a backup for steady state operation but at increased cost due to its lower efficiency while operating at a small fraction of maximum capacity.

Regardless of the location of the System Test Facility, various miscellaneous equipment will have to be procured, including, in particular, a vacuum insulated cold gas storage tank for helium gas generated during quenches.

\section{Power Supply}

Both BNL and FNAL have existing power supplies that can be used for initial testing. However, the capabilities of these supplies support different aspects of the test program. The power supply at FNAL allows $7 \mathrm{kA}$ peak current for ramps at any rate $(500 \mathrm{~V} \max )$, but thermal constraints in the supplies limit the rms current to $4.6 \mathrm{kA}$. DC operation at 7 
$\mathrm{kA}$ is limited to approximately $2 \mathrm{hr}$ by the thermal time constant and rms current rating of the transformer.

BNL can provide this same current range with a single power supply rated at $5 \mathrm{kA}$, but at a ramp rate limited by the $50 \mathrm{~V}$ maximum output. BNL can parallel two of these supplies to provide a continuous dc capability of $10 \mathrm{kA}$.

To provide the total test capability for any of the magnet types, both laboratories must obtain additional power supplies. To test the long string of type A or B magnets, FNAL must purchase a $50 \mathrm{~V}, 7 \mathrm{kA}$ holding supply and a new $7 \mathrm{kA}$ filter choke. BNL must procure a supply capable of approximately $200 \mathrm{~V}$ and $7 \mathrm{kA}$ peak current.

To test the type $C$ magnets, either laboratory would have to purchase power supplies to provide the dipole requirements, $12 \mathrm{kA}$ at $120 \mathrm{~V}$, and $6 \mathrm{kA}$ at $60 \mathrm{~V}$, and the quad requirements, $2.5 \mathrm{kA}$ at about $100 \mathrm{~V}$.

Both laboratories have an inventory of minor power supplies to use for powering trim coils, etc. Neither laboratory is likely to have a complete set of the required supplies, so some additional power supplies will have to be purchased.

\section{Control, Logging and Simulation Systems}

In this category, the approaches of the two laboratories, which are based on existing equipment, are markedly different. FNAL has in place a complete capability to serve the superconducting Energy Saver accelerator. This system can be expanded to include the Long Test Facility for initial operation. It could also act as an umbrella system into which SSC controls development can be integrated.

BNL has commercial process control systems that they propose to use for monitoring and controlling both test strings. Software and hardware effort will be required to adapt this computer system for initial and continuing string operation.

\section{Quench Detection and Protection}

The detailed requirements for quench detection and protection depend on the magnet type chosen. The FNAL proposal would use slightly modified Energy Saver equipment. This equipment has a complete hardware and software interface into FNAL's proposed control system and can initially provide all of the quench detection, protection, system control and data archival functions presently provided in the Energy Saver. The BNL system is less well developed and requires some design effort prior to first tests. BNL plans to use local analog comparison of magnet differential voltage signals for quench detection and to provide two independent paths for operation of the dump via the SCR switch and the dc breaker.

\section{Utilities and Space}

The facilities at BNL generally meet the requirements for both space and utilities. This is principally because the unused CBA tunnel, which is highly suitable for the long string tests, is available. This tunnel is outfitted with all the utilities required for the long string 
tests. Its cross section is larger than that envisioned for the SSC tunnel, but demarcation or simple physical barriers of a section of the CBA tunnel would realistically simulate the working space expected to be available in the SSC tunnel. Two platforms and one enclosure would have to be erected to cross the experimental areas. The adjacent buildings would provide more than adequate office and workshop space.

The BNL proposal to use existing floor area and utility man-trenches in the former Cosmotron experimental building (B905) for the short string test appears satisfactory. The type $\mathrm{C}$ magnet would have to be tested straight and the test facility would extend into the adjacent building (905). Minimal effort would be required to supply the needed power, refrigeration and utilities.

At FNAL, the enclosures for the long string and part of the short string tests would have to be built from the ground up. The long string would be located along Kautz Rd. extending to the south from near service building E4 of the Main Ring. For this test, a level concrete pad would be poured and an inexpensive prefabricated building would be installed on the east side of the road to provide the required enclosure. Temporary buildings or "Porta-Kamps" would be set up to provide work areas and office space at the head of the string near E4. All utilities would have to be installed. They would tie into existing utilities at or near the Main Ring. Main Ring tunnel sections will be placed on the Industrial Building 1 parking lot as an enclosure for the short string tests. This proposed enclosure would extend to the west from the MTF building and use utilities located in that area.

\section{Manpower}

The manpower requirements for construction and operation of the STF at BNL and FNAL are shown in Table V.7. Breakdowns of the total manpower requirements into that required for the Long String Facility and for the Short String Facility are shown in Tables V.8 and V.9, respectively. The principal conclusions that may be drawn from these tables are as follows:

1. There is little difference in the total manpower estimates of the two laboratories for any of the three magnet types;

2. Each Laboratory would require approximately the same total professional manpower effort (i.e., 31-35 man years) during the expected lifetime of the STF;

3. BNL would require approximately $50 \%$ more technical manpower effort than FNAL (i.e., $\sim 72$ man years vs $\sim 48$ man years) mainly because of greater needs for the operation of the cryogenic system, and in connection with engineering and software for the power supply and control systems.

Both BNL and FNAL would draw key personnel for the STF from other Laboratory programs and possibly from other national laboratories. In addition, assuming that present programs continue and new programs materialize as planned, it is very likely that the location of the STF at either BNL or FNAL would require the designated Laboratory to supplement its staff by outside hiring. 


\section{Costs}

The cost estimates for the STF, generated by the two laboratories, and described in detail in Section V and Appendices D and E, are summarized in Tables VI.1 through VI.5. Though the cost of civil construction is higher at FNAL than at BNL, this is more than offset by the higher cost of power supplies and controls at BNL as shown in Table VI.2.

The operating costs at BNL are slightly higher than those at FNAL, mainly because of the need to support a larger staff to operate the refrigerators and the higher cost of power, as shown in Table VI.3.

The bottom line costs for the STF at the two laboratories are very close; and the differences are probably within the uncertainties that stem from the assumptions on which the estimates are based.

\section{E. General Remarks on STF Site Selection}

The process of selecting a site for the Systems Test Facility cannot be considered final until an agreement satisfactory to the managements of the SSC and the host laboratory has been successfully negotiated. This agreement must encompass a large number of separate understandings including such diverse items as: identification of key staff assigned to the facility; space allocations during the life of the program; the applicable overhead rate structure; and priorities in the use of laboratory shops and other facilities. 
Table VI.1

Total construction and operating costs* $(\mathrm{k} \$)$ of the STF during the four year duration of the SSC string tests at BNL and FNAL.

\begin{tabular}{|c|c|c|c|c|c|c|}
\hline & \multicolumn{3}{|c|}{ BNL } & \multicolumn{3}{|c|}{ FNAL } \\
\hline & A & B & $\mathrm{C}$ & A & $\mathrm{B}$ & $\mathrm{C}$ \\
\hline & \multicolumn{3}{|c|}{ Construction Costs } & & & \\
\hline $\begin{array}{l}\text { Short String } \\
\text { Long String }\end{array}$ & $\begin{array}{r}845 \\
2655\end{array}$ & $\begin{array}{r}850 \\
2710\end{array}$ & $\begin{array}{r}851 \\
2770\end{array}$ & $\begin{array}{r}618 \\
2298\end{array}$ & $\begin{array}{r}616 \\
2506\end{array}$ & $\begin{array}{r}641 \\
2768\end{array}$ \\
\hline \multirow[t]{2}{*}{ Total } & 3500 & 3560 & 3621 & 2916 & 3122 & 3409 \\
\hline & \multicolumn{3}{|c|}{ Operating Costs } & & & \\
\hline $\begin{array}{l}\text { Short String } \\
\text { Long String }\end{array}$ & $\begin{array}{l}1443 \\
3709\end{array}$ & $\begin{array}{l}1019 \\
3354\end{array}$ & $\begin{array}{l}1047 \\
3289\end{array}$ & $\begin{array}{l}1135 \\
3822\end{array}$ & $\begin{array}{r}710 \\
3312\end{array}$ & $\begin{array}{r}760 \\
3367\end{array}$ \\
\hline \multirow[t]{2}{*}{ Total } & 5152 & 4373 & 4336 & 4957 & 4022 & 4127 \\
\hline & \multicolumn{3}{|c|}{ Total Costs } & & & \\
\hline $\begin{array}{l}\text { Short String } \\
\text { Long String }\end{array}$ & $\begin{array}{l}2288 \\
6364\end{array}$ & $\begin{array}{l}1869 \\
6064\end{array}$ & $\begin{array}{l}1898 \\
6059\end{array}$ & $\begin{array}{l}1753 \\
6120\end{array}$ & $\begin{array}{l}1326 \\
5818\end{array}$ & $\begin{array}{l}1401 \\
6135\end{array}$ \\
\hline Grand Total & 8652 & 7933 & 7957 & 7873 & 7144 & 7536 \\
\hline
\end{tabular}

*The costs in this table are for comparison purposes only and should not be used for budget estimates. 
Table VI.2

A summary of total construction costs* (k\$)for the System Test Facilities for the three magnet types at Brookhaven National Laboratory and Fermilab during the four year duration of the tests

\begin{tabular}{|c|c|c|c|c|c|c|}
\hline \multirow{2}{*}{ Item } & \multicolumn{3}{|c|}{ BNL } & \multicolumn{3}{|c|}{ FNAL } \\
\hline & A & B & $\mathrm{C}$ & A & B & $\mathrm{C}$ \\
\hline Civil Construction & 152 & 152 & 152 & 708 & 708 & 730 \\
\hline Cryogenics & 740 & 725 & 716 & 665 & 665 & 665 \\
\hline Power Supplies & 1418 & 1493 & 1788 & 545 & 771 & 946 \\
\hline Controls & 672 & 672 & 672 & 292 & 292 & 292 \\
\hline Safety & 33 & 33 & 33 & 20 & 20 & 20 \\
\hline Installation & 485 & 485 & 260 & 450 & 450 & 550 \\
\hline Test Coordination & & & & 150 & 150 & 150 \\
\hline Miscellaneous & & & & 45 & 45 & 45 \\
\hline Cryogenic Fluids & & & & 41 & 21 & 11 \\
\hline Total & 3500 & 3560 & 3621 & 2916 & 3122 & 3409 \\
\hline
\end{tabular}

*The costs in this table are for comparison purposes only and should not be used for budget estimates. 
Table VI.3

A summary of total operating costs* for the System Test Facilities for the three SSC magnet types at Brookhaven National Laboratory and Fermilab during the four year duration of the string tests

\begin{tabular}{|c|c|c|c|c|c|c|}
\hline \multirow{2}{*}{ Item } & \multicolumn{3}{|c|}{$\mathrm{BNL}$} & \multicolumn{3}{|c|}{ FNAL } \\
\hline & $\mathrm{A}$ & B & $\mathrm{C}$ & A & B & $\mathrm{C}$ \\
\hline Cryogenics & 1686 & 1691 & 1691 & 1064 & 927 & 927 \\
\hline Power Supplies & 740 & 655 & 655 & 800 & 790 & 940 \\
\hline Control & 258 & 236 & 236 & 45 & 30 & 30 \\
\hline \multicolumn{7}{|l|}{ Safety } \\
\hline Installation & - & - & - & 360 & 360 & 360 \\
\hline Test Coordination & 534 & 474 & 474 & 585 & 570 & 570 \\
\hline Miscellaneous & & & & 185 & 165 & 165 \\
\hline Cryogenic Fluids & 634 & 322 & 310 & 980 & 480 & 435 \\
\hline Power & 1300 & 995 & 970 & 938 & 700 & 700 \\
\hline Total & 5152 & 4373 & 4336 & 4957 & 4022 & 4127 \\
\hline
\end{tabular}

*The costs in this table are for comparison purposes only and should not be used for buidget estimates. 
Table VI.4

Costs* (k\$)for the Short String Test for the

three magnet types at Brookhaven National Laboratory and Fermilab

\begin{tabular}{|c|c|c|c|c|c|c|}
\hline \multirow{2}{*}{ Item } & \multicolumn{3}{|c|}{ BNL } & \multicolumn{3}{|c|}{ FNAL } \\
\hline & A & B & $\mathrm{C}$ & A & B & $\mathrm{C}$ \\
\hline Civil Construction & & & & 20 & 20 & 20 \\
\hline Cryogenics & 525 & 530 & 530 & 732 & 595 & 595 \\
\hline Power Supplies & 644 & 564 & 594 & 115 & 105 & 130 \\
\hline Controls & 150 & 128 & 128 & 105 & 90 & 90 \\
\hline Safety & 4 & 4 & 4 & & & \\
\hline Installation & 58 & 58 & 29 & 85 & 85 & 110 \\
\hline Test Coordination & 228 & 168 & 168 & 75 & 60 & 60 \\
\hline Miscellaneous & & & & 80 & 60 & 60 \\
\hline Cryogenic Fluids & 174 & 82 & 110 & 278 & 136 & 161 \\
\hline Power & 505 & 335 & 335 & 263 & 175 & 175 \\
\hline Total & 2288 & 1869 & 1898 & 1753 & 1326 & 1401 \\
\hline
\end{tabular}

*The costs in this table are for comparison purposes only and should not be used for budget estimates. 
Table VI.5

Summary Costs* (k\$)for the Long String Test for the three magnet types at Brookhaven National Laboratory and Fermilab

\begin{tabular}{|c|c|c|c|c|c|c|}
\hline \multirow{2}{*}{ Item } & \multicolumn{3}{|c|}{ BNL } & \multicolumn{3}{|c|}{ FNAL } \\
\hline & A & B & $\mathrm{C}$ & $\mathbf{A}$ & B & $\mathrm{C}$ \\
\hline Civil Construction & 152 & 152 & 152 & 688 & 688 & 710 \\
\hline Cryogenics & 1901 & 1886 & 1877 & 997 & 997 & 997 \\
\hline Power Supplies & 1514 & 1584 & 1849 & 1230 & 1456 & 1756 \\
\hline Controls & 780 & 780 & 780 & 232 & 232 & 232 \\
\hline Safety & 29 & 29 & 29 & 20 & 20 & 20 \\
\hline Installation & 427 & 427 & 231 & 725 & 725 & 800 \\
\hline Test Coordination & 306 & 306 & 306 & 660 & 660 & 660 \\
\hline Miscellaneous & & & & 150 & 150 & 150 \\
\hline Cryogenic Fluids & 460 & 240 & 200 & 743 & 365 & 285 \\
\hline Power & 795 & 660 & 635 & 675 & 525 & 525 \\
\hline Total & 6364 & 6064 & 6059 & 6120 & 5818 & 6135 \\
\hline
\end{tabular}

*The costs in this table are for comparison purposes only and should not be used for budget estimates. 


\title{
Appendix A
}

\author{
Membership of the \\ Task Force On SSC Magnet System Test Site
}

D. Brown
G. Cottingham
R. Louttit
E. Willen

P. Vander Arend

D. Bogert

P. Limon

R. Orr

C. Rode

A. Tollestrup

G. Tool

W. Hassenzahl

R. Neal, Chairman

R. Huson
Brookhaven National Laboratory Brookhaven National Laboratory Brookhaven National Laboratory Brookhaven National Laboratory

Cryogenics Consultants Inc.

Fermi National Accelerator Laboratory Fermi National Accelerator Laboratory Fermi National Accelerator Laboratory Fermi National Accelerator Laboratory Fermi National Accelerator Laboratory Fermi National Accelerator Laboratory

Lawrence Berkeley Laboratory

Stanford Linear Accelerator Center

Texas Accelerator Center 


\title{
Appendix B
}

\author{
Task Force Charge
}

\section{Introduction}

It has been anticipated that thorough testing of the proposed SSC magnet system components in a system setting will be necessary prior to construction of the SSC. In view of the relatively long lead time needed to prepare for and carry out the test, it seems appropriate to examine the question of where to carry out the test. There seems to be wide agreement that the test site can be independent of magnet style and of place of magnet manufacture. There also seems to be wide agreement that, by virtue of existing facilities and experience, BNL and FNAL are the two best candidate sites. Consequently it seems appropriate to restrict our considerations to those possibilities at this time.

In determining the most advantageous venue for the test, both cost effectiveness and availability of the needed facilities and manpower are primary factors. In assessing what facilities are needed, it is necessary to have a clear concept of the scope of the system(s) to be tested, of the types and numbers of tests to be carried out and of the likely length of time for which this initial system test facility must be available.

\section{Report}

Wanted is a report with a critical evaluation of the two potential sites with regard to availability of the needed facilities and manpower on the needed time scale and an estimate of the cost to designated SSC R/D funds of preparing each of the sites for the tests and for operating the facilities during the test period. It seems reasonable to assume that the cost of installing the magnets and other components to be tested will be relatively site independent and consequently need not be considered in the report unless this hypothesis is believed by the Task Force to be incorrect. In writing your report you should assume that adequate magnets and other components will be available early in CY 1987 and that all initial major design validation tests need to be completed in CY 1987.

This report, dealing with the technical aspects of the SSC Magnet Systems test, will be used as a major part of the input in deciding the most appropriate venue and management for these Magnet System Tests.

The Task Force Report should contain 6 major items:

1. A description of a suggested Test System(s) for each of the three magnet styles of the RDS. For example, in the case of design A it might be 5 lattice cells together with all cryogenic distribution, power supply, control and monitor equipment needed for accelerator operation or alternatively it might be two cryogenically independent groups of $\mathrm{X}$ cells for simultaneous testing of different features, etc., etc. 
2. A description of a suggested test regimen(s) for the suggested Test System(s). For example, one might need $10^{\mathrm{n}}$ full energy ramps at rate $\mathrm{X} \mathrm{Ts}{ }^{-1}, 10^{\mathrm{m}}$ simulated beam-induced quenches, $10^{p}$ cool down-warm up cycles with interspersed magnetic, mechanical and electrical measurements, in situ magnetic field measurements, quench propagation across all boundaries, etc., etc. Estimate the time required to carry out each of the suggested major design validation tests. It seems reasonable to assume that a major system test bed facility needs to be in operation throughout $\mathrm{Ph} \mathrm{I}$ and $\mathrm{Ph}$ II of the SSC and that it is practical and desirable to move the system test bed to the SSC construction site at the end of the first year after NTP. Criticize that assumption and the consequent conclusion that the test facilities under discussion in your report need to be available until the end of the first year after NTP even though the first round of design validation tests are to be completed at the end of 1987.

3. A description with rough specifications of the major facilities needed to carry out the suggested tests. To be included are equipment, housing needs, personnel work space, refrigeration, cryogenic distribution, power supplies, utilities and personnel and equipment safety systems. Any significant dependence of facility needs on magnet style should be described and cost differences estimated.

4. A list for both BNL and FNAL of the relevant facilities that exist and a critical evaluation of their suitability for the task at hand including a description of needed modifications, if any. Also, for both FNAL and BNL, list additional facilities that would have to be purchased or built to carry out the test program. For both potential sites estimate the cost to designated SSC R/D funds for modification of existing facilities and for bringing them into operation in readiness for the tests as well as the cost to SSC R/D funds to provide and bring into operation any additional facilities that would be required.

5. An estimate for both sites of the manpower required to prepare the facilities for the tests and to operate the facilities during the test. The estimate should be broken down in terms of physicist, engineer, technician, and other manpower. Assess the availability of the needed manpower at each of the sites.

6. Discuss other technical and economic factors which may affect the cost and availability of needed resources on the needed time scale and compare the two sites with respect to these factors. 


\title{
Appendix C
}

\author{
Meeting Agenda \\ Task Force on SSC Magnet System Test Site
}

September 13-14, 1984

SLAC Orange Room, Central Laboratory

Sept. 13

(Thursday)

$0900 \quad$ Executive Session

0915 General Comments and Advice to Task Force Maury Tigner

$0930 \quad$ Magnet A: Proposed test system, test program, facilities and staffing

Bob Louttit

et al.

1100 Magnet B: Proposed test system, test program, facilities and staffing

Rich Orr et al.

1230 Lunch (in meeting room)

1330 Magnet C. Proposed test system, test program, facilities and staffing

Russ Huson

et al.

1500 General Discussion and Reconciliation of Plans

$\sim 1730 \quad$ Adjournment

Sept. 14

(Friday)

0900 Completion of written "specifications" for test facilities, program, and staffing

$1230 \quad$ Adjournment 
C-2

Tentative Agenda Meeting of

Task Force on SSC Magnet System Test Site

September 25, 1984

FNAL "Black Hole," $2^{\text {nd }}$ Floor, Wilson Hall

0700 Review and edit latest drafts of report sections:

Section II - Description of Test Systems

Section III - Test Program

Section IV - Major Facilities Required

0800 Working breakfast in meeting room

0830 FNAL response to test facility "specifications"

1100 Visit proposed test facility area

1230 Lunch in meeting room

1330 Continue discussion of FNAL response and edit for report purposes

1500 Adjourn - travel to O'Hare for flight to Islip, L.I. 
Tentative Agenda Meeting of

Task Force on SSC Magnet System Test Site

September 26-27, 1984

BNL

Building 902 A, Room 63

Sept.26

(Wednesday)

0830

Administrative Session

0930

BNL response to test facility "specifications"

1200

Lunch in meeting room

1300

Visit proposed test facility area

1430

Continue discussion of FNAL and BNL responses and edit for report purposes

$\sim 1730$

Adjourn*

*Note: Depending on progress made, an evening session may be required.

Sept. 27

(Thursday)

0830 Discuss Task Force conclusions - prepare and edit for report (Section VII)

$1200 \quad$ Lunch in meeting room

1300 Continue editing entire report

$\sim 1730 \quad$ Adjourn 
C-4

Tentative Agenda Meeting of

Task Force on SSC Magnet System Test Site

October 11-12, 1984

O'Hare Hilton Hotel — Room 2091

Oct. 11

(Thursday)

0830

Review and edit Section $V$ of report

(summaries of BNL and FNAL responses and

Summary Tables from BNL/FNAL)

1100 Review and edit Section VI (Conclusions)

$1230 \quad$ Lunch

1330 Prepare summary of report

$1600 \quad$ Continue discussion and editing

$\sim 1730 \quad$ Adjourn

Oct. 12

(Friday)

$0830 \quad$ Review and edit entire report

$1230 \quad$ Lunch

$1330 \quad$ Continue review and editing

$\sim 1700 \quad$ Adjourn 


\section{APPENDIX D \\ BROOKHAVEN NATIONAL LABORATORY \\ RESPONSE TO THE TEST FACILITY SPECIFICATIONS}

\section{INTRODUCTION}

Brookhaven National Laboratory proposes to use its resources to assemble and operate a System Test Facility for the Superconducting Super Collider. The primary purpose of the STF would be to test a large group of magnets and associated cryostats, interconnects, spool pieces, lead pots, etc. configured as closely as possible to the current SSC design. Every attempt would be made to provide a system allowing the addition of new subsystems (controls, power supplies, etc.) for testing as their designs are finalized. However, the plans and cost estimates outlined in this proposal are aimed only at producing an STF ready for magnet system testing at the earliest desired time and at the lowest possible cost.

The STF Task Force has set forth performance specifications in Section III of their report. Two test configurations are required to operate simultaneously, a Short String and a Long String. The test procedures for each have different objectives, resulting in steady state and non-steady state loading conditions which are quite different. The Short String will be one SSC halfce11 in length ( 100 to $140 \mathrm{~m}$ depending on magnet type) and the Long String will reach its ful1 length at about $1.2 \mathrm{~km}$. The two prime considerations in choosing sites at BNL for the tests are (1) string length and (2) availability of cryogenic facilities.

Table 1 describes the helium refrigerators which could play some part in the operation of these tests. Many of these refrigerators will be underutilized or even idle for the next few years unless devoted to SSC testing. The way in which BNL proposes to site these tests is based on our current best estimate of 
Table 1

\section{HELIUM REFRIGERATOR DESIGN PARAMETER SUMMARY}

\begin{tabular}{|c|c|c|c|c|c|c|}
\hline & & DESIGN & PRAMETERS & & & $\begin{array}{c}\text { PRIMARY PLANNED USE IN FUTURE } \\
\text { PROGRAMS }\end{array}$ \\
\hline SYSTEM & CAPACITY & $\begin{array}{l}\mathrm{LN} \\
\mathrm{REQ} \\
?\end{array}$ & $\begin{array}{l}\text { COMPR. } \\
\text { FLOW } \\
\mathrm{g} / \mathrm{s} \\
\end{array}$ & $\begin{array}{l}\text { EXPAND. } \\
\text { TYPE }\end{array}$ & $\begin{array}{l}\text { DELIV. } \\
\text { METHOD }\end{array}$ & \\
\hline Mode1 1400 & $100 \mathrm{~W} @ 4.5 \mathrm{~K}$ & Yes & 14 & Recip. & $\begin{array}{l}\text { Pool } \\
\text { Boiling }\end{array}$ & Lumited use - no compressors \\
\hline Model 2000 & $175 \mathrm{~W} @ 4 / 5 \mathrm{~K}$ & Yes & 25 & Recip. & $\begin{array}{l}\text { Forced } \\
\text { Flow }\end{array}$ & Heat Load Measurements of Prototype Components \\
\hline $\begin{array}{l}7 \text { 'Bubble } \\
\text { Chamber } \\
\text { Mode1 } 4000\end{array}$ & $700 \mathrm{~W} @ 4.5 \mathrm{~K}$ & Yes & 81 & Recip. & $\begin{array}{l}\text { Pool } \\
\text { Boiling }\end{array}$ & $\begin{array}{l}\text { Pool Bolling Tests - Primarily SSC Conductor } \\
\text { Short Sample Tests }\end{array}$ \\
\hline PAT & $\begin{array}{l}700 @ 4.5 \mathrm{~K}+ \\
4 \mathrm{~g} / \mathrm{s} \text { vapor } a \\
4.5 \mathrm{~K}\end{array}$ & Yes & 128 & Turbo & $\begin{array}{l}\text { Pool } \\
\text { Boiling }\end{array}$ & $\begin{array}{l}\text { Vertical Dewar Pool Boiling } 5 \mathrm{~m} \text { Magnet Tests } \\
\text { followed by SSC Forced-Flow Production Magnet }\end{array}$ \\
\hline $\begin{array}{l}\text { MAGCOOL } \\
\text { Mode1 } 4000\end{array}$ & $1580 \mathrm{~W} d 4.5^{\circ} \mathrm{K}$ & Yes & 158 & Recip. & $\begin{array}{l}\text { Forced } \\
\text { F1ow }\end{array}$ & SSC Short String Magnet Test \\
\hline$R \& D$ & $\begin{array}{l}840 \mathrm{~W}+2 \mathrm{~g} / \mathrm{s} \\
\text { e } 4.5 \mathrm{~K} \\
\text { or } \\
1400 \mathrm{~W}+2 \mathrm{~g} / \mathrm{s} \\
\text { @ } 4.5 \mathrm{k}\end{array}$ & Yes & 100 & Recip. & $\begin{array}{l}\text { Forced } \\
\text { Flow }\end{array}$ & SSC Long String Test \\
\hline RHIC & $\begin{array}{l}9350 \mathrm{~W}<3.8 \mathrm{~K}+ \\
4300 \mathrm{~W}<4.2 \mathrm{~K}+ \\
100 \mathrm{~g} / \mathrm{s} \text { त } 3.8 \mathrm{~K}+ \\
55,000 \mathrm{w}<69 \mathrm{~K}\end{array}$ & No & 4100 & Turbo & $\begin{array}{l}\text { Forced- } \\
\text { Flow }\end{array}$ & $\begin{array}{l}\text { Cooldown of SSC Long String and liquid as } \\
\text { required for SSC testing. Eventually for } \\
\text { RHIC }\end{array}$ \\
\hline
\end{tabular}


the most suitable location and equipment for each test. Other combinations are possible and could be substituted if engineering studies so indicate.

We propose to house the magnets of the Long String in the RHIC tunne1, proceeding counterclockwise from just beyond the injection area at $6 o^{\prime} \mathrm{clock}$ for $1.2 \mathrm{~km}$. The R\&D Refrigerator and the RHIC Refrigerator would be used in concert to provide cooling for the magnets. The Collider Center will be utilitized for technician work areas, offices and other facilities.

Two power supplies bought for CBA would be connected in paralle1 to provide the required current for the Long String. Since they are rated at only 50v, they will not be capable of ramping the full Long String at the fast ramp rate desired, but would be capable of doing so for the early partial string testing. Another similar supply is available for the Short string testing. After the magnet decision is made, power supplies, switchgear and other equipment appropriate for the Long String testing can be ordered for delivery around the end of FY-1986. The equipment will be installed and tested we 11 in advance of the start of Long String tests in $\backsim$ January 1987. However, essential1y a 11 of the equipment necessary to begin testing on both strings already exists at BNL.

It is our belief that this proposal meets the requirements of the STF specification generated by the Task Force on SSC Magnet System Test Site. The schedule in the specification can be met with comfortable margins. The eventual expected use for the tunnel, Collider Center and refrigerator is for the Relativistic Heavy Ion Collider (RHIC). However, the most optimistic schedule for its construction shows that no interference with the STF could be expected before about the middle of FY-1990. Thus, the STF could operate for $\wedge 1.5$ years after the nominal scheduled completion of magnet system tests. By that time it is reasonable to expect that the first refrigerator and tunnel section would be avail- 
D-4

able at the SSC construction site for final system testing, and that no further use of the STF would be desired.

The Short String Test would be located in Building 902 and would use the MAGCOOL facility for cooling. The details of the proposed arrangements for both tests are given in the following sections. 


\section{LONG STRING TEST}

Site

The long, unobstructed length of space available in the RHIC tunnel makes that a natural choice for the site of the Long String Test. Two helium refrigerators are located along the tunnel in such a way that both can be used to suit various different operating modes which will be encountered during the Long String Test program. The R\&D refrigerator is located toward the 5 o'clock side of the Injection Area at 6 o'clock. See Figure 1 (Overa11 Site Plan) and Figure 2 (Sextant $5 \mathrm{Plan}$ View). Figure 1 has a distance scale shown along the tunnel with zero at the R\&D refrigerator.

Whatever magnet is chosen, the Long String would be installed in the tunnel starting at the R\&D refrigerator and extending counterclockwise for $1.2 \mathrm{~km}$. The tunne 1 maximum width is 16 feet, maximum height $\sim 11$ feet and the full circumference is $3.8 \mathrm{~km}$. An interior view is shown in Figure 3. It is complete with lighting, air conditioning, emergency ventilation system, $480 / 220 / 120 \mathrm{~V}$ power and instrument air. The Long string would have to cross the 4 o'clock Open Area. There, special magnet supports to accomodate a 3 foot floor elevation change and a temporary enclosure would be required for a distance of 188 feet. Special magnet supports will also be required at the 2 o'clock Experimental Area to compensate for the 18 inch lower floor elevation.

About 200 meters from the start of the string, the center alcove of the 5 o'clock sextant is reached. Here is located the the Collider Center for nearby works areas and other personnel space; the RHIC refrigerator together with its compressor station, cooling tower, high pressure gas storage and computer control system, and numerous pieces of vacuum and miscellaneous equipment. A plan 
D-6

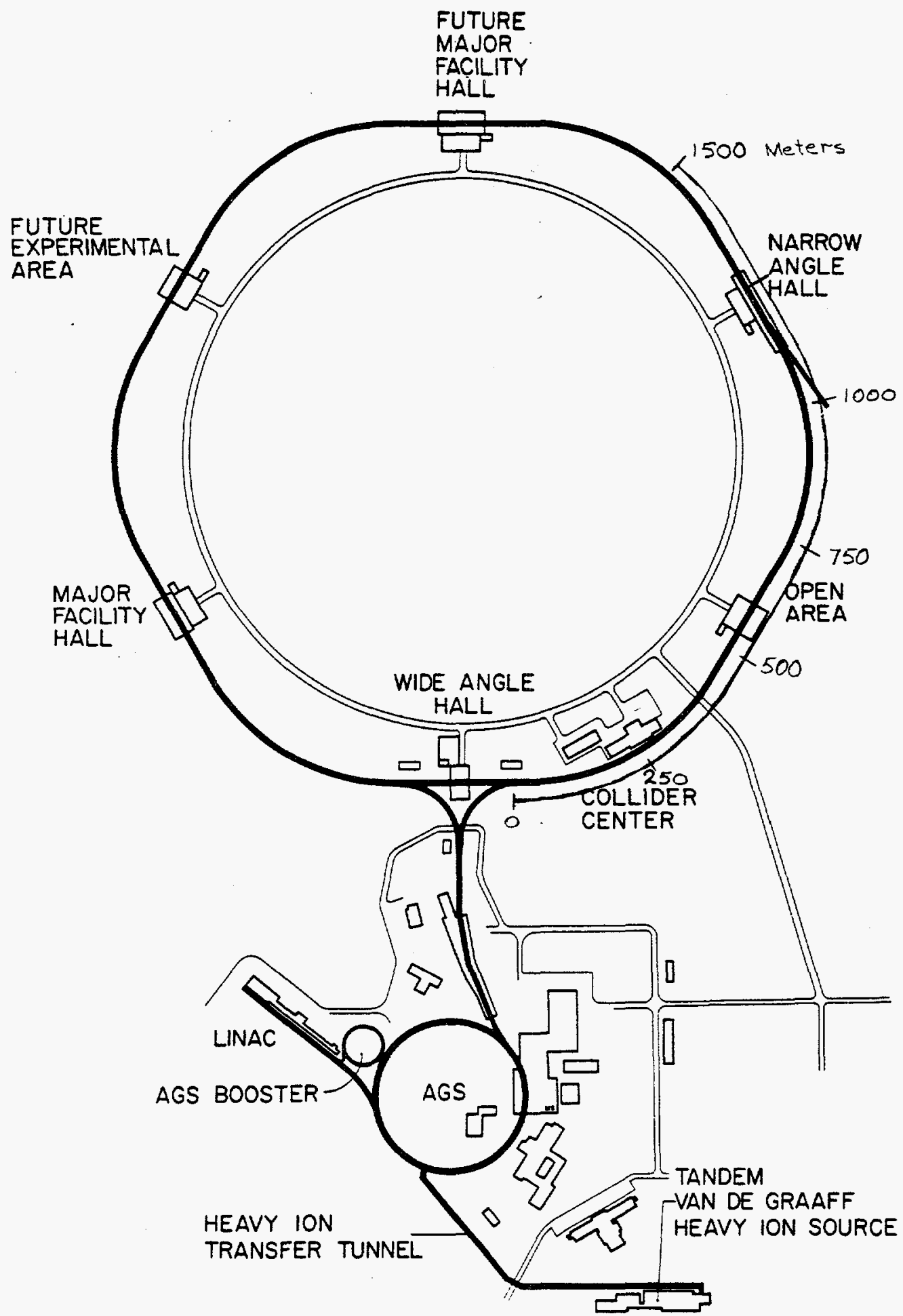

Figure 1: Overall Site Plan 
D-7

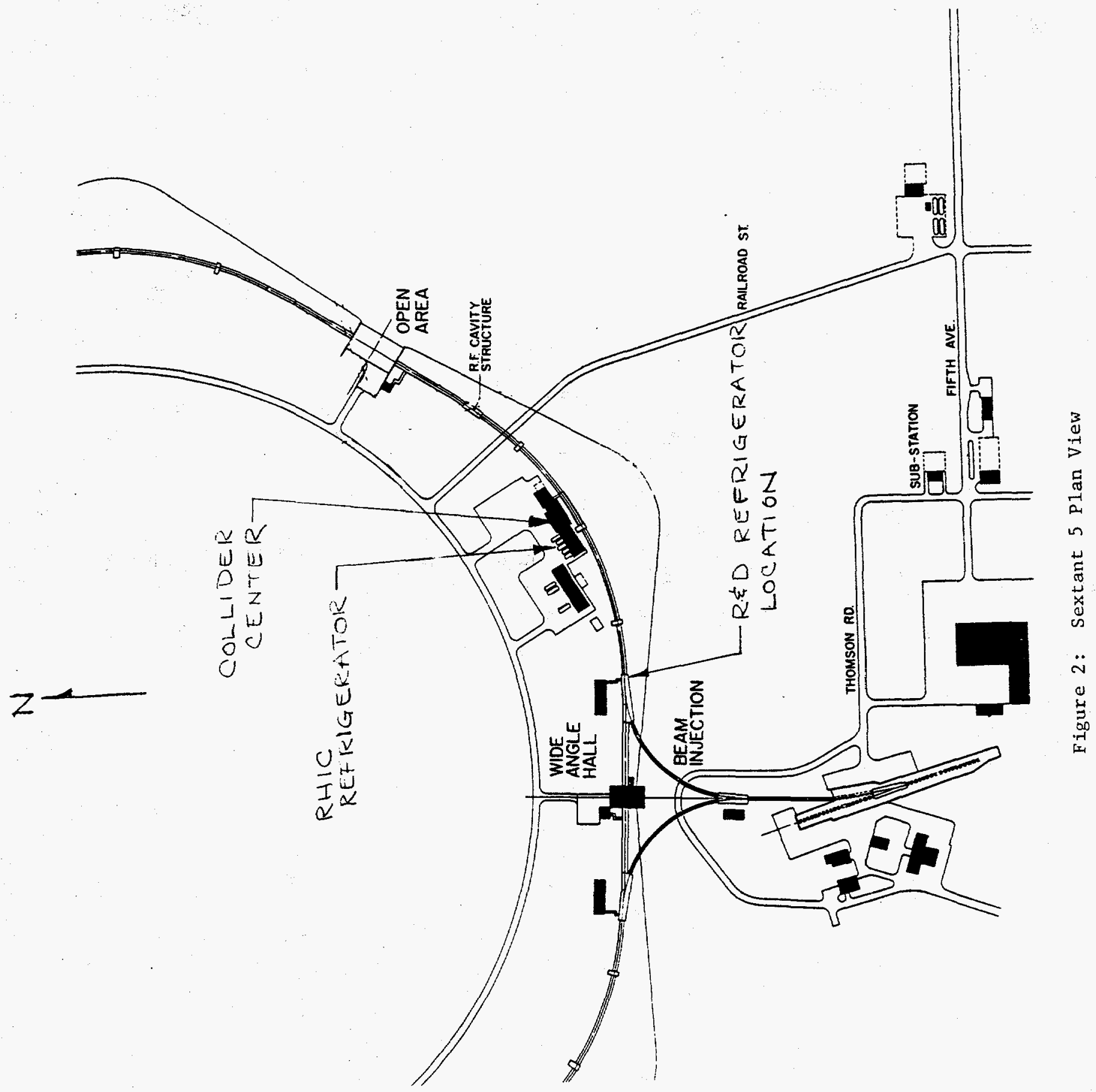


D-8

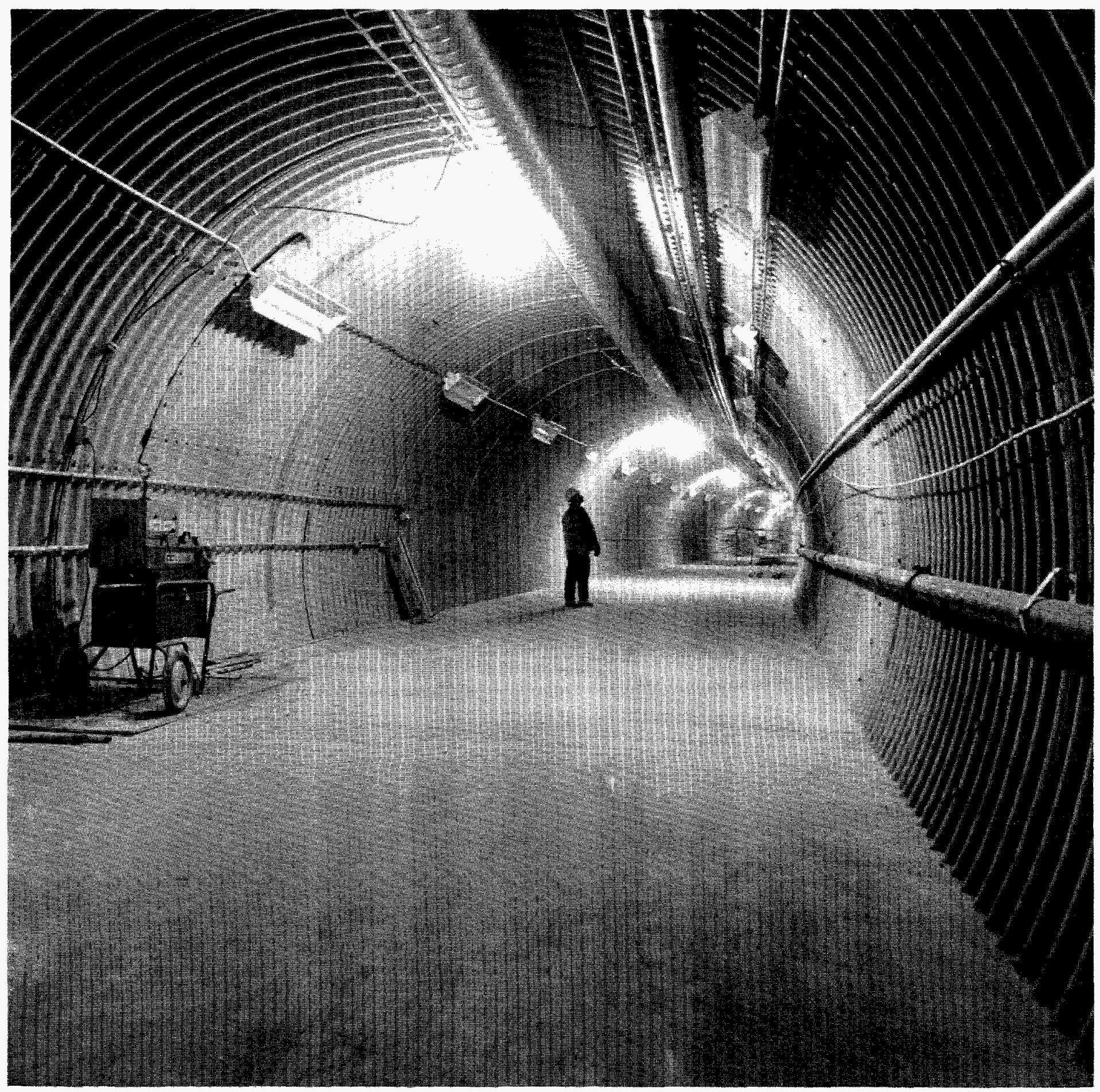

Figure 3

View of Tunnel Interior 
view of the structures which would comprise the center of STF operations is shown in Figure 4.

The Collider Center contains two adjacent technician works areas of $\checkmark 600$ sq. ft. each which are available for this purpose. It also contains $7000 \mathrm{sq}$. $\mathrm{ft}$. of computer floor of which more than the $600 \mathrm{sq}$. ft. required by the spec. can be devoted to the development of SSC controls later if desired. However, on day one, the control system installed originally for the CBA cryogenic system will be used with certain modifications and additions as described later. These existing controls are located in an air conditioned control room of $\sqrt[360]{ } \mathrm{sq}$. ft. in the Cryogenic Building. Office space can be partitioned off as desired in the Collider Center, which is otherwise finished and contains air conditioning, sanitary facilities, etc.

$\underline{\text { Refrigeration }}$

The R\&D refrigerator will be used to supply refrigeration to the Long String during most of the test program. The RHIC refrigerator will be used in order to speed the cooldown of the magnets from room temperature. If load conditions are such that more refrigeration is required than can be supplied by the $R \& D$ Refrigerator in a stand-alone mode, this refrigerator could be operated in a satellite mode. If it were required to operate in the satellite mode, the RHIC refrigerator would be used to produce liquid helium which would be stored and used, as required, by the R\&D refrigerator to increase its capacity.

The RHIC refrigerator is in the final stage of field installation. Pressure checking of process lines in both buildings is under way, first tests of compressors are expected in December, and the entire system should have completed acceptance tests by summer 1985. This leaves a comfortable period of more than one year before the earliest need for system tests. Since completion 
D-10

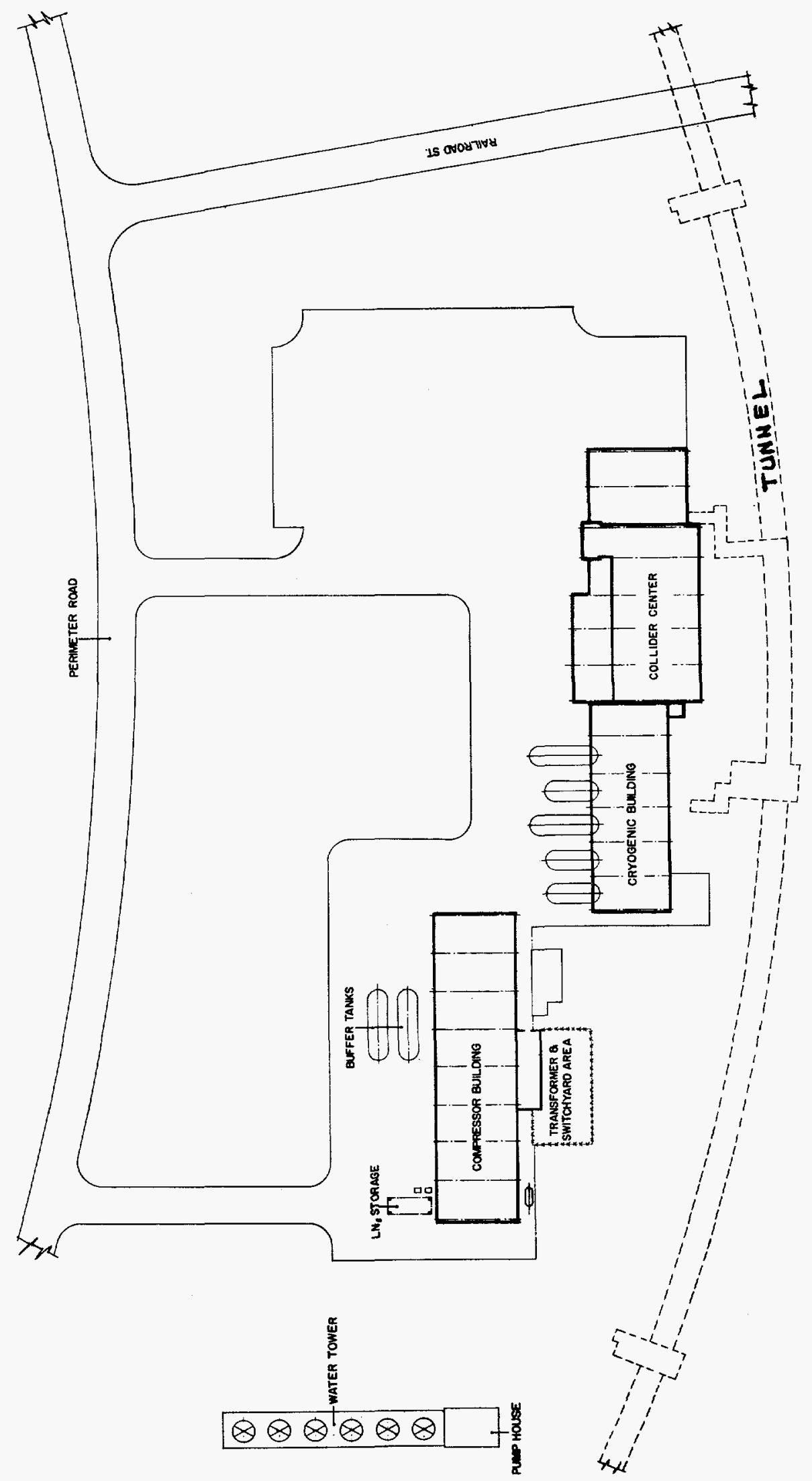

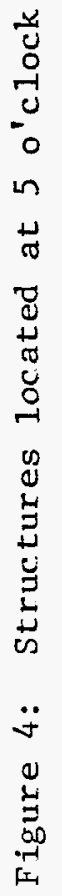


through acceptance tests is part of the manufacturer's obligation under his contract with BNL, commissioning of this refrigerator cannot become a burden on SSC funds .

The R\&D refrigerator is located in the tunnel as shown in Figure 5 . It was operated there to cool the CBA Full Cell Test. The cold box contains not only the heat exchangers usually found in a helium refrigerator, but in addition houses two liquid-to-gas heat exchangers and (by means of suitable ports) a cold ( $\sqrt{4}$ K) vacuum pump and a cold circulating compressor. This permits considerable flexibility in the flow rate and temperature conditions at which this refrigerator can supply helium to a load.

Each magnet type requires different equipment configurations to create conditions in the Long String which are similar to those needed in the SSC for that magnet type. Figure 6 shows a configuration of the low temperature end of the R\&D refrigerator which would create Magnet A conditions. Figure 7 shows Magnet $\mathrm{B}$ at $4.5 \mathrm{~K}$ and Figure 8 shows Magnet $\mathrm{B}$ arranged for lower temperature operation. Figure 9 shows an equipment configuration for Magnet $C$.

Three Sullair screw compressors are part of the $R \& D$ refrigerator system. Each compressor will draw about $180 \mathrm{~kW}$ of electric power when operated. With two compressors operating (See the T-S Cycle Diagram in Figure 10) this refrigerator will produce $840 \mathrm{~W}$ of refrigeration at $4.5 \mathrm{~K}$ while $2 \mathrm{~g} / \mathrm{s}$ of liquid is being withdrawn for lead cooling. This is sufficient refrigeration to satisfy the design heat load requirements including cold compressor operation for any of the magnet types. If run essentially flat out in the stand-alone mode with all three compressors on line the refrigerator can produce $1400 \mathrm{~W}+2 \mathrm{~g} / \mathrm{s}$. This is the mode in which it would be operated during the period ( $s \mathrm{hr}$ ) required to re- 
D-12

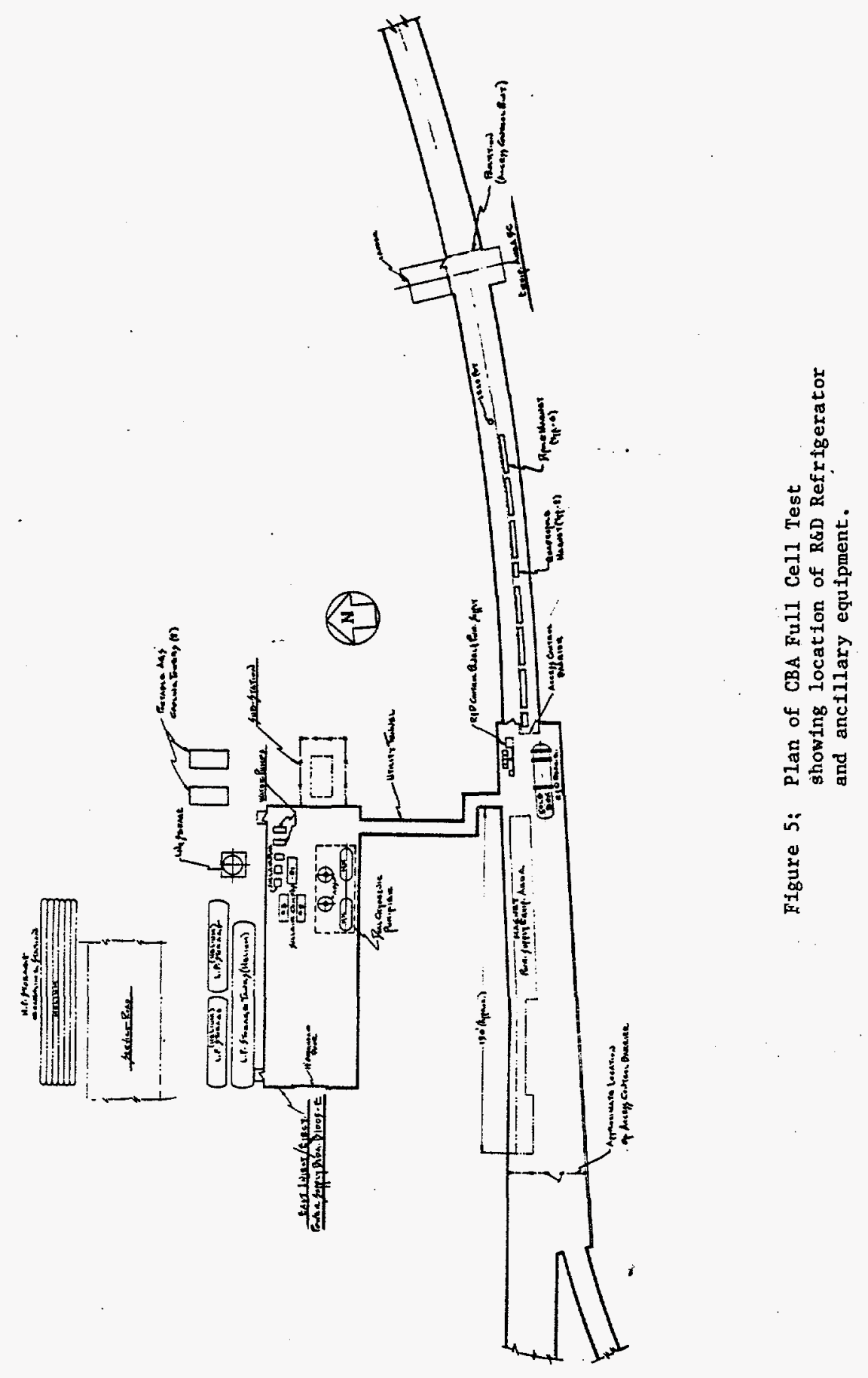


D-13

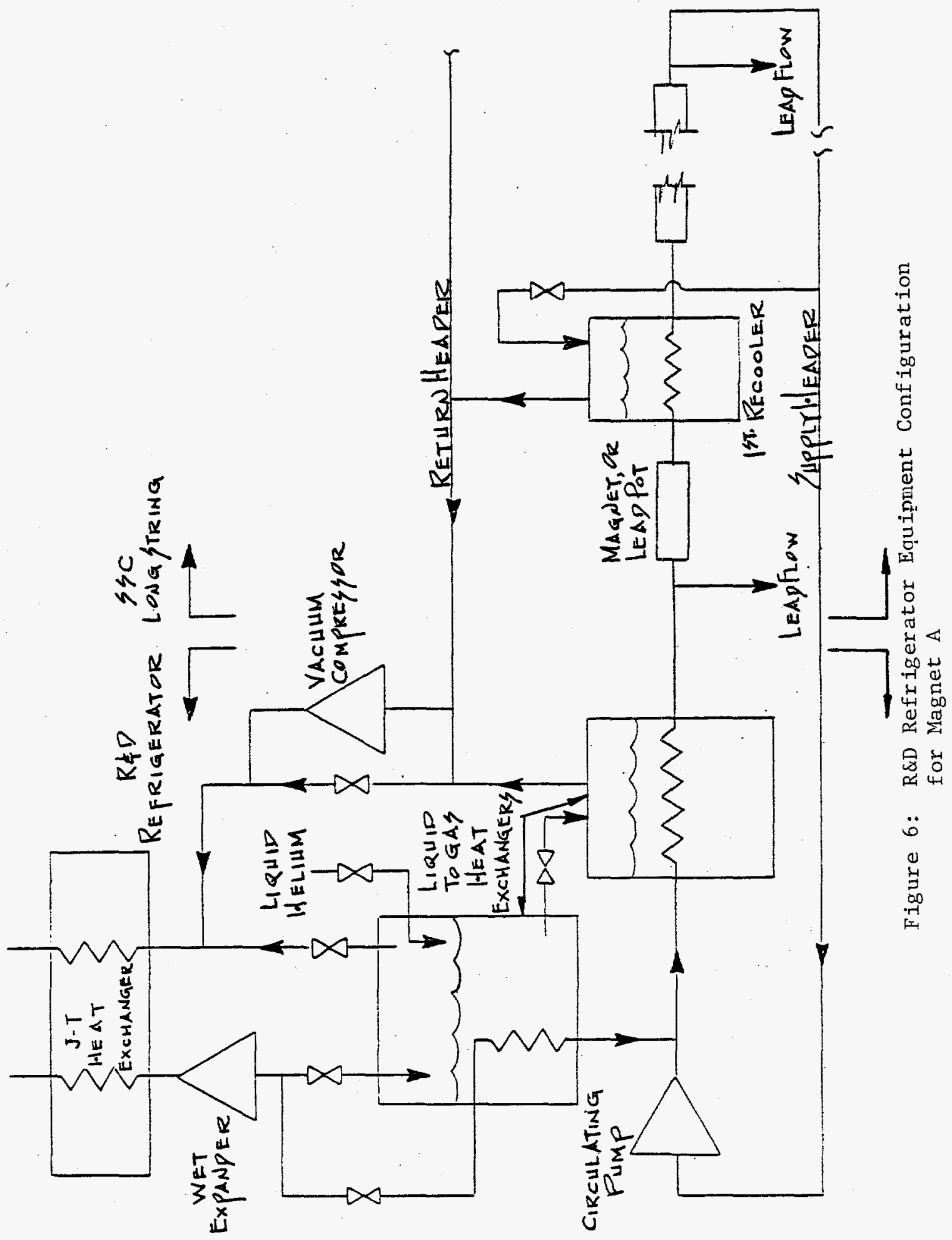




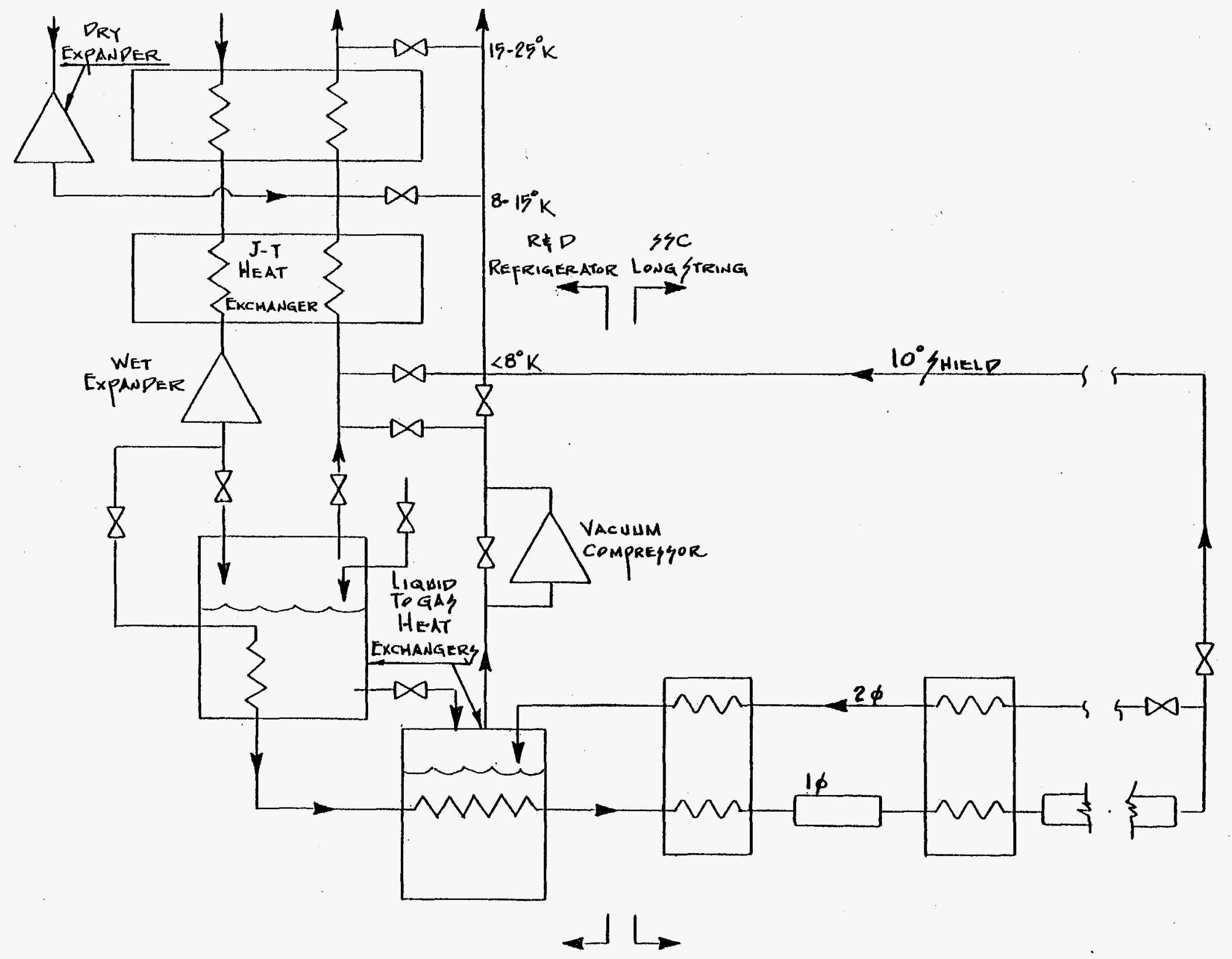

Figure 7: R\&D Refrigerator Equipment Configuration for Magnet B, $4.5 \mathrm{~K}$ 
D-15

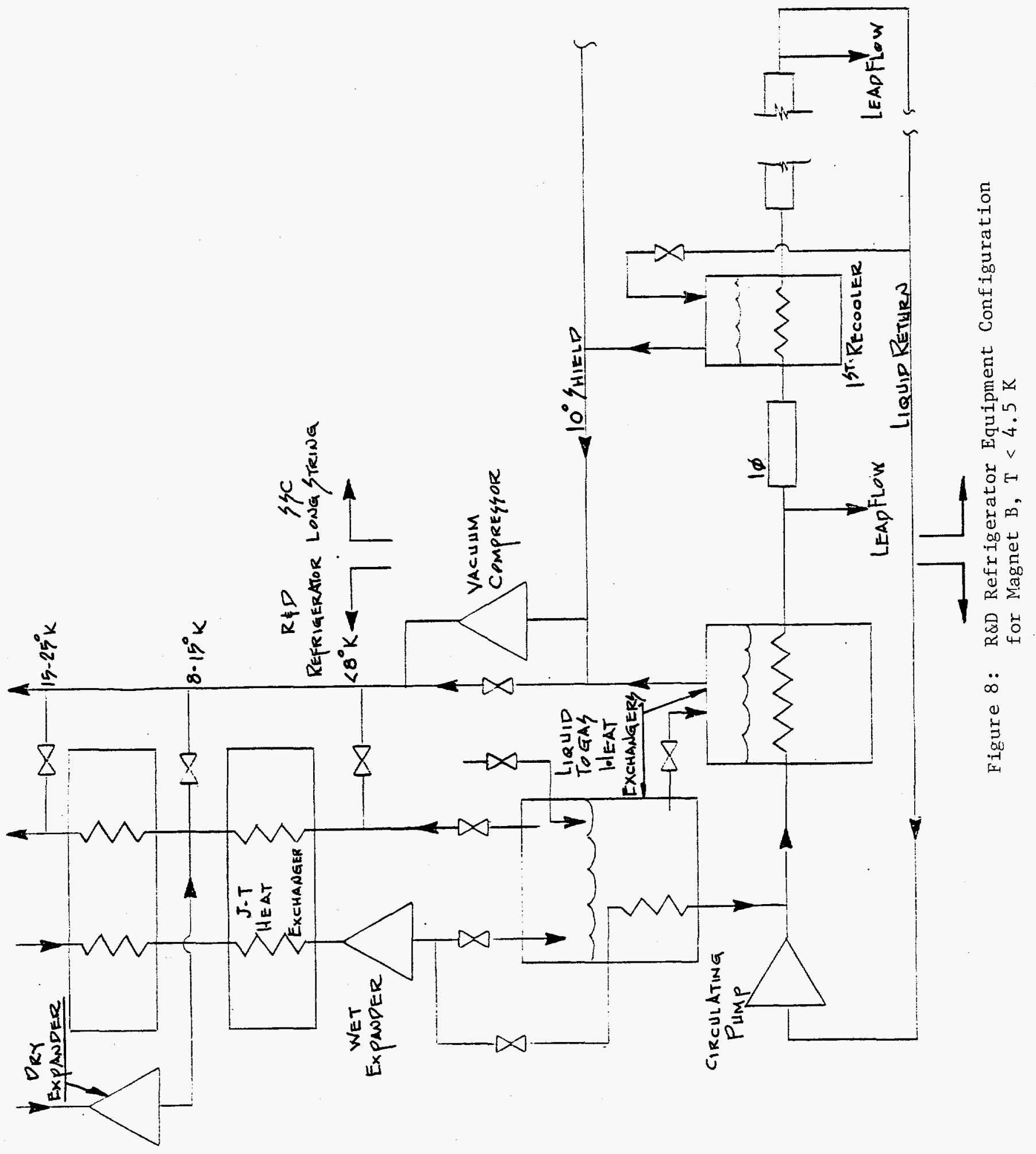


D-16

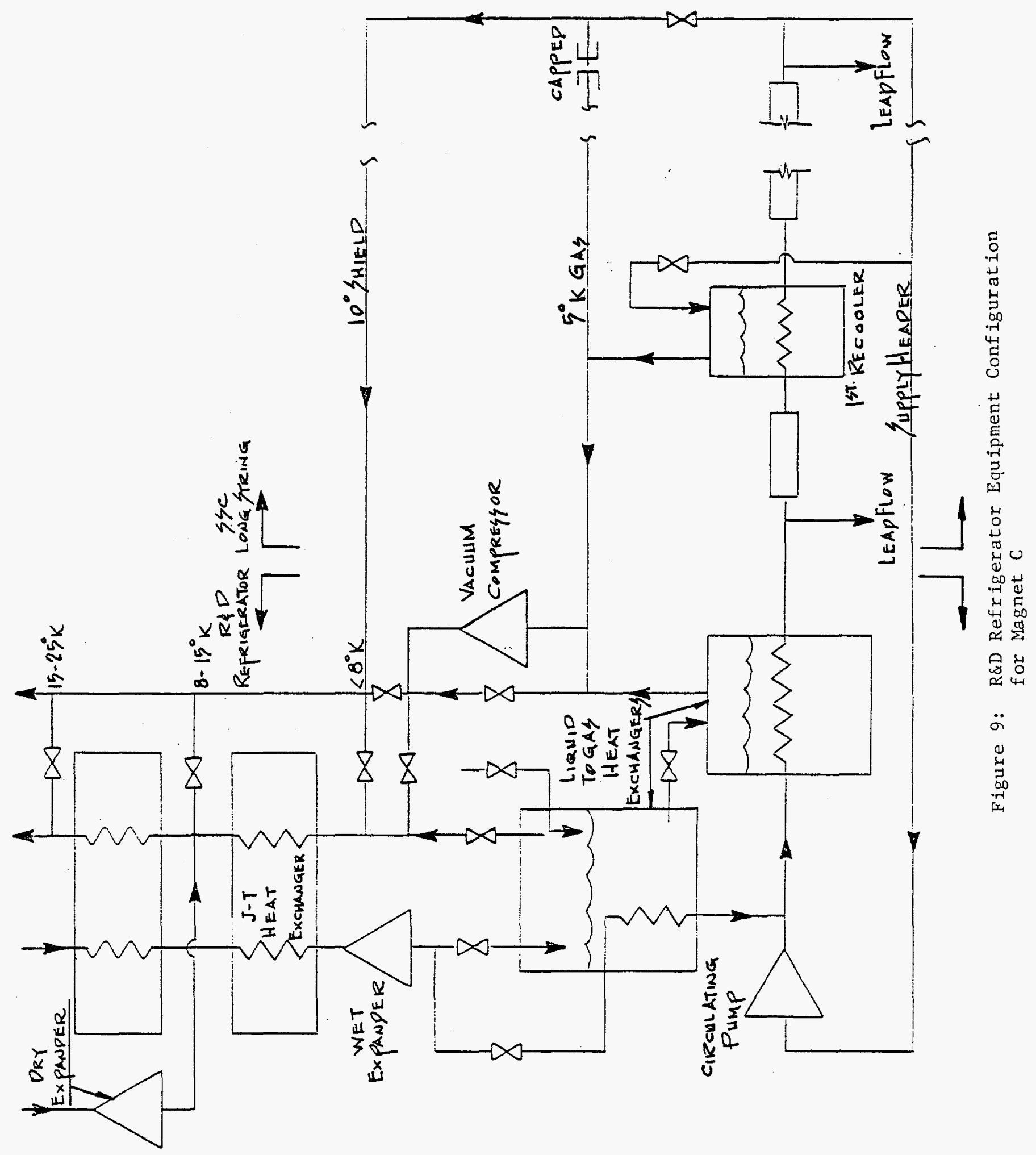


D-17

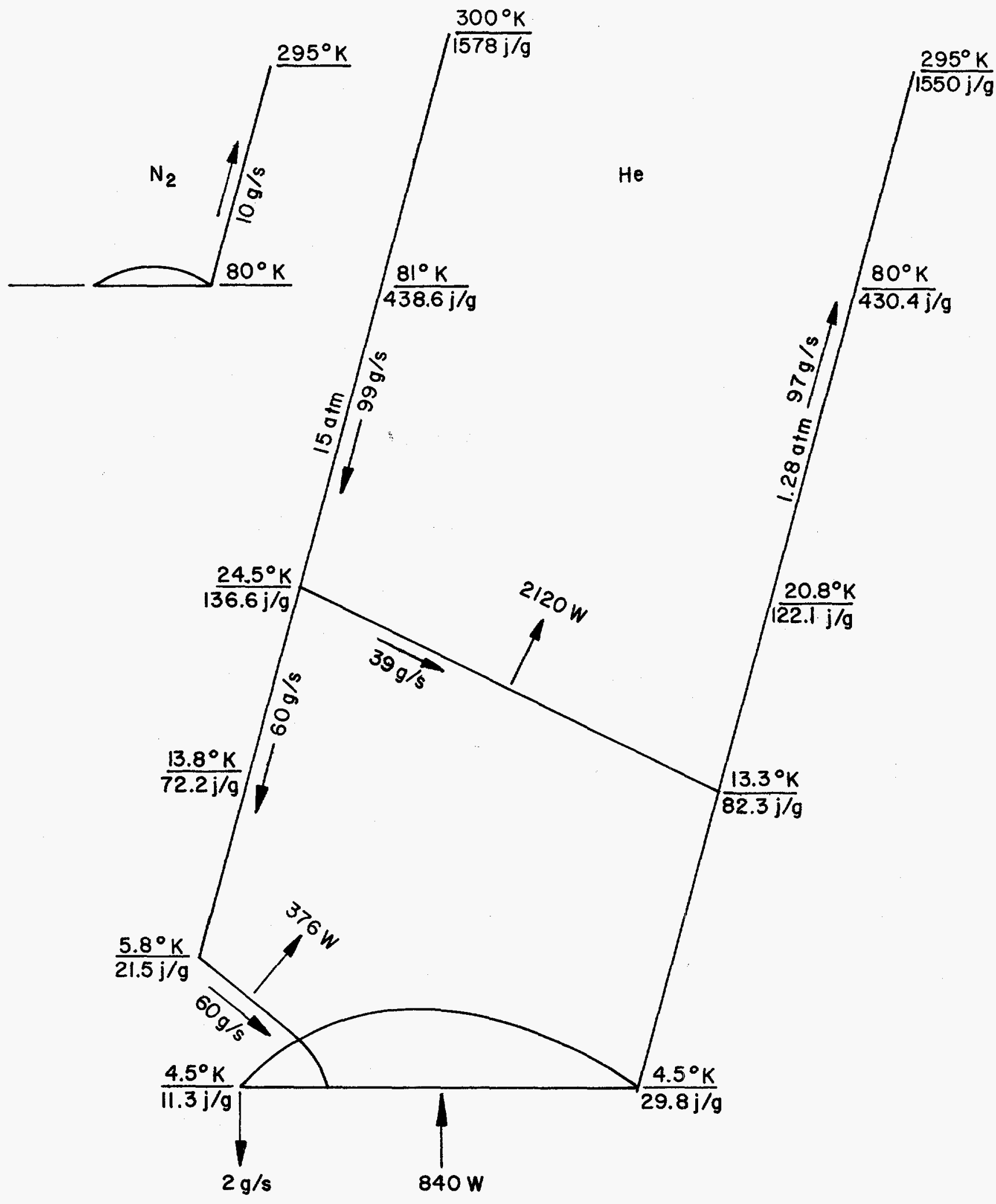

Figure 10: T-S Diagram

R\&D Refrigerator $100 \mathrm{~g} / \mathrm{s}$ Compressor Flow Stand-alone Mode 
cover from a quench. The calculated state points for this compressor flow rate are indicated on the T-S coordinates of Figure 11.

Liquid helium can be added to the refrigerator to further increase the capacity. This is the same cycle as that run by the FNAL satellite refrigerators. We calculate that approximately $1600 \mathrm{~W}$ to $1700 \mathrm{~W}$ is the maximum capacity using any reasonable liquid injection rate. The amount of liquid which can be withdrawn for lead cooling is a function of the liquid helium injection rate. The results of a sample calculation are shown in Figure 12. The liquid injection rate is $14.7 \mathrm{~g} / \mathrm{s}$ and the lead cooling is taken at $6.2 \mathrm{~g} / \mathrm{s}$. The refrigeration produced below $4.5 \mathrm{~K}$ is $1712 \mathrm{~W}$. Because the return flow rate exceeds the supply rate, the return temperatures run very low. More load (a shield?) could be managed at temperatures above $4.5 \mathrm{~K}$. No liquid nitrogen or dry expander flow is required in this mode. The small increases in output (200-300W) operating in the satellite mode should not be required by the Long string. Because some considerable cost ( $\$ 100 \mathrm{~K}$ capital cost, $\$ 50 \mathrm{~K} / \mathrm{yr}$. dewar rental and $\$ 350 \mathrm{~K} / \mathrm{yr}$. power cost) is involved in providing this extra margin, we are not proposing to provide the equipment to operate in this mode. If need for the capacity should arise at a later date, the equipment could be added.

The cold box of the R\&D refrigerator contains two liquid-to-gas heat exchangers and many cold valves. With a minimum of effort the piping can be rearranged to suit the desired cycle. A $150 \mathrm{~g} / \mathrm{s}$ cold circulating compressor (and spare) has been used extensively in the CBA Full Cell tests and is available for use on the Long String. These machines were built by Creare, Inc. of Hanover, N.H. and run on gas bearings. A $53 \mathrm{~g} / \mathrm{s}$ cold vacuum pump built by Rotoflow on oil bearings has also been used and is available if required. 


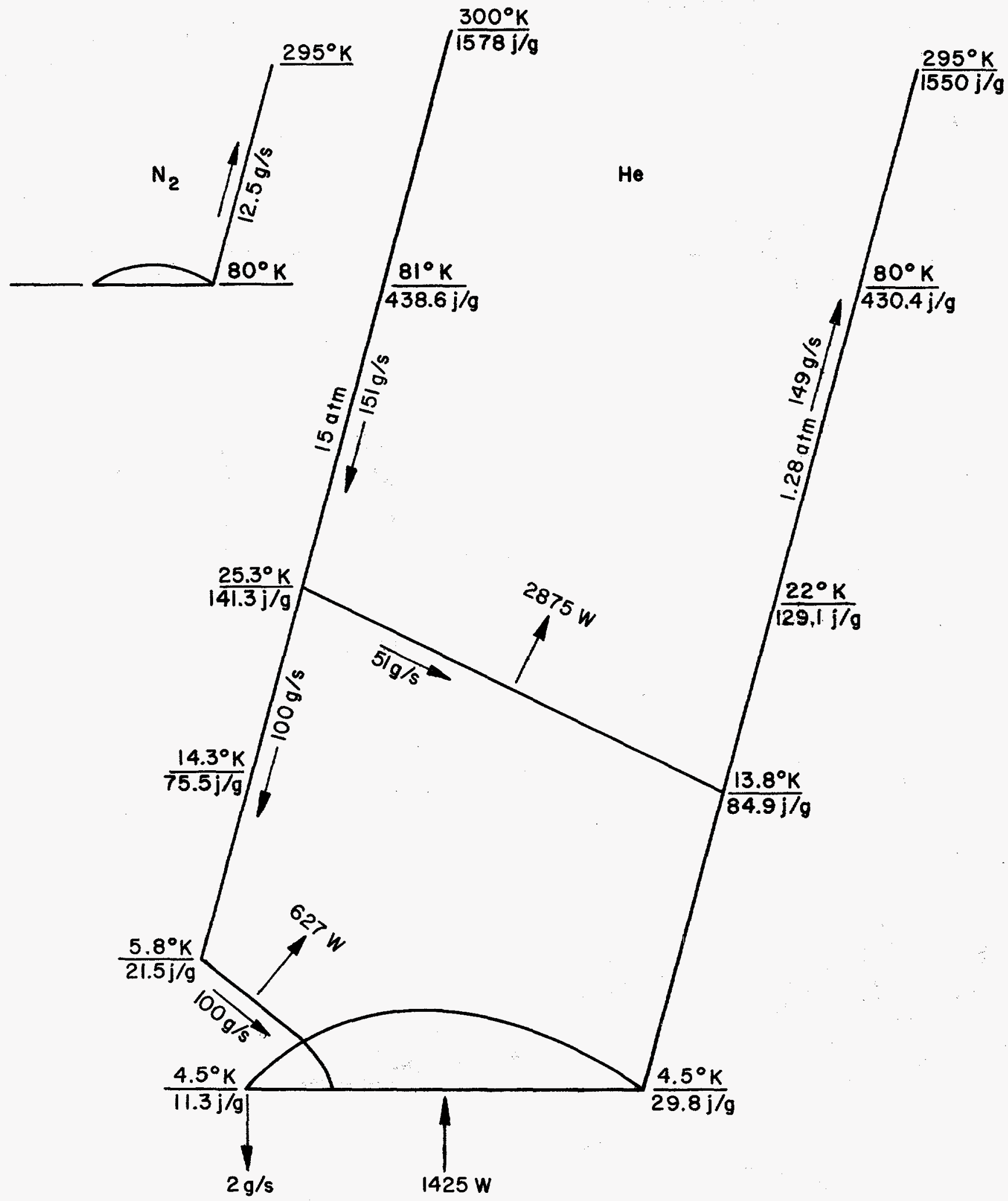

Figure 11: T-S Diagram

R\&D Refrigerator $151 \mathrm{~g} / \mathrm{s}$ Compressor Flow Stand-alone Mode 
D-20
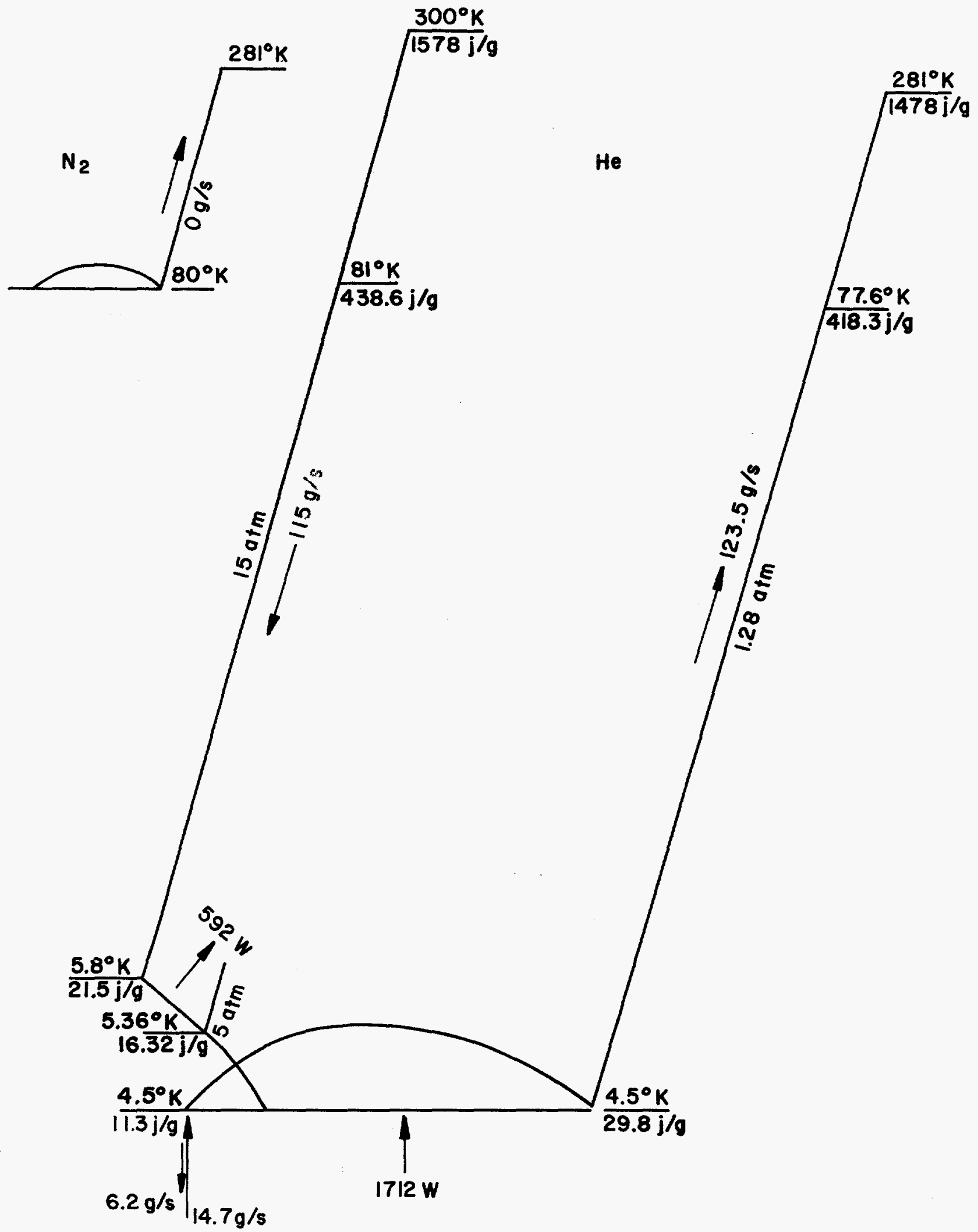

Figure 12: T-S Diagram

R\&D Refrigerator Satellite Mode Sample Calculation 
Rotoflow also built a $172 \% / \mathrm{s}$ cold circulating compressor which has been received by $B N L$, but not tested.

BNL proposes to use the helium refrigerator originally intended for ISABELLE/CBA to provide the cold helium required for cooldown of the Long String Tests. The schematic for the baseline refrigerator design is shown in Figure 13. Figures 14 and 15 show the calculated values for the baseline design point for this refrigerator. When operating at this point the refrigerator can simultaneously produce the following output:

Liquid: $100 \mathrm{~g} / \mathrm{s}(3000 \mathrm{liter} / \mathrm{hr})$.

Refrigeration: $6.6 \mathrm{~kW}$ at $2.5 \mathrm{~K}$

$13.2 \mathrm{~kW}$ at $3.3 \mathrm{~K}$

$55 \mathrm{~kW}$ at $55 \mathrm{~K}$

If operated as a liquefier only, the theoretical liquid output is $375 \mathrm{~g} / \mathrm{s}$ $(\mathcal{M}, 250$ liter/hr.). Because this is an off-design point for the plant, this output would probably never be realized and a capacity of 10,000 liters/hr. would be a more realistic expectation.

A paper which describes the analysis of the cooldown performance of the RHIC (then ISABELLE) Refrigerator is attached. The results of the analysis show that a cooldown flow of $410 \mathrm{~g} / \mathrm{s}$ at a temperature of $13.1 \mathrm{~K}, 15.5 \mathrm{~atm}$ can be attained. No liquid nitrogen is required. These conditions would permit the removal of heat from the Long String at the rate of $626.3 \mathrm{~kW}$. While the supply pressure is ample, the flow rate is so high that pressure drop in the Long String magnets may be a limitation to the cooling rate. The calculated cooldown time for the Long String with magnets A,B or C is, respectively, $1.1,0.3$ and 0.4 days. An additional 0.1 day would be required to fill at $200 \mathrm{~g} / \mathrm{s}$ in each 


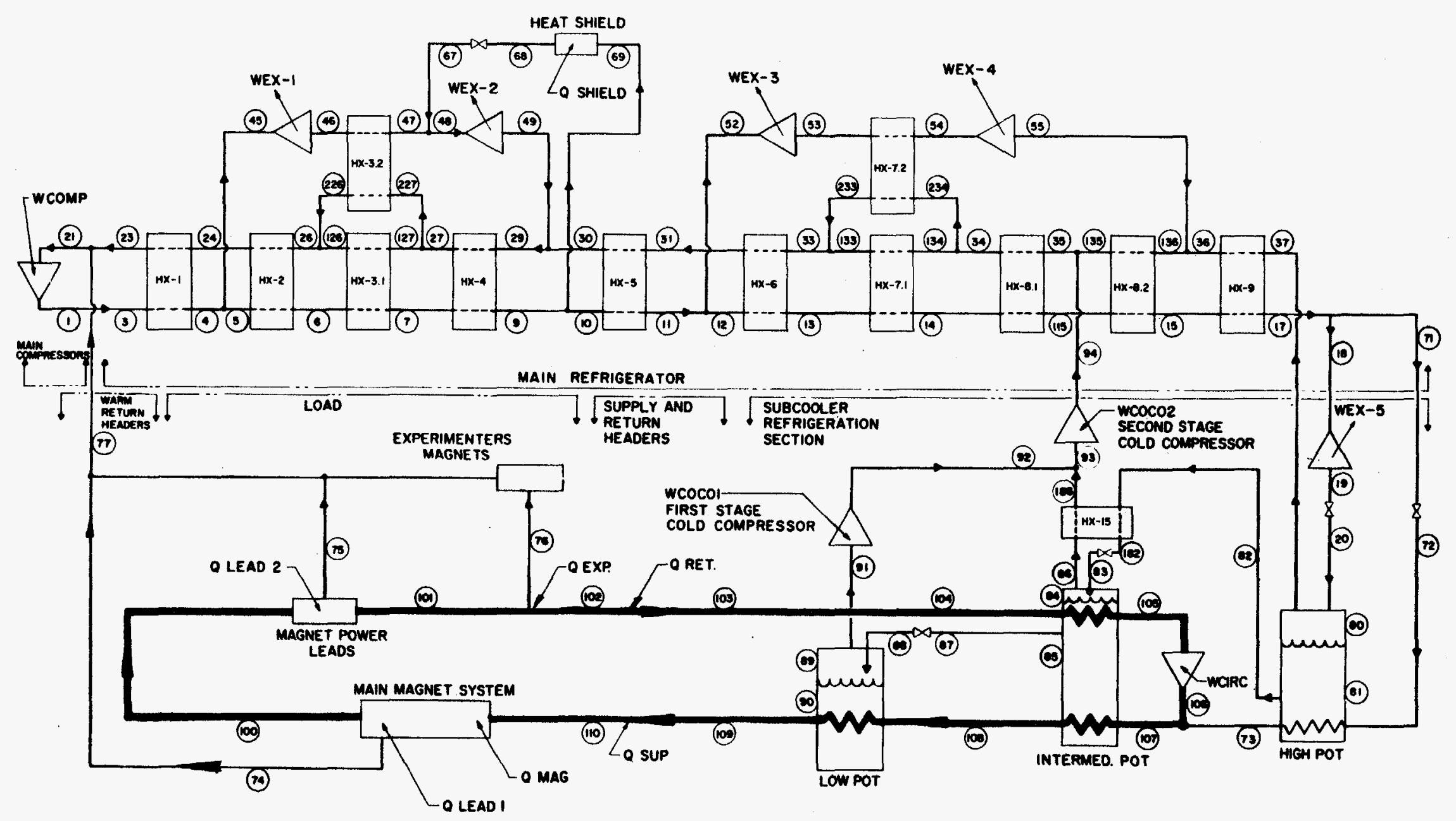

REFRIGERATOR FLOW SHEET

Figure 13 
CALCULATED PERFORMANCE OF A HELIUM REFRIGERATOR WHICH UTILIZES 5 EXPANDERS AND 3 COLD COMPRESSORS. DEL IVERY OF THE REFRIGERANT IS IN THE FORM OF COMPRESSED LIQUID HELIUM WHICH IS CIRCULATED BY ONE OF THE THREE COMPRESSORS IN THE CYCLE.

SUMMARY OF SYSTEM PARAMETERS

SYSTEM DESIGN STEADY-STATE LOAD REQUIREMENTS

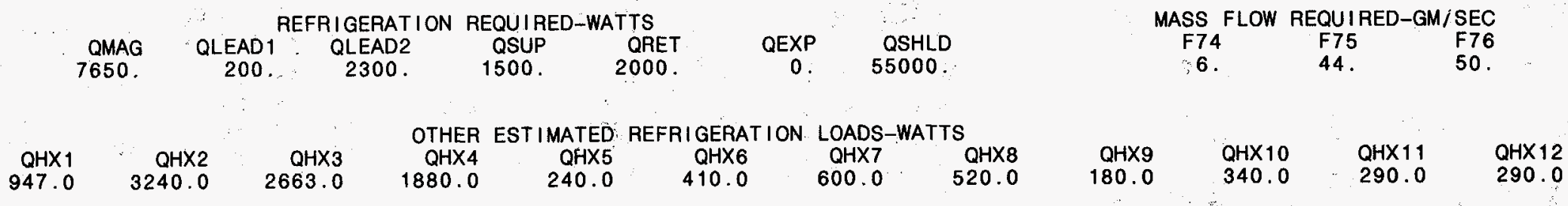

EXPANDER/COMPRESSOR PARAMETERS

ADIABATIC EFFICIENCY I SOTHERMAL EFFICIENCY INLET PRESSURE-ATM OUTLET PRESSURE-ATM INLET TEMPERATURE-K OUTLET TEMPERATURE FLOW RATE-GM/SEC WORK-WATTS WORK-HP

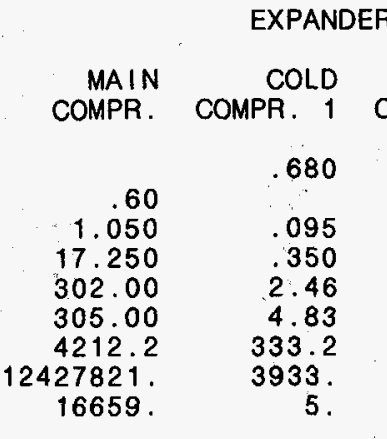
COLD
COMPR. 2 CIRC EXPANDER EXPANDER EXPANDER EXPANDER EXPANDER
5

.670
340
1.389
4.61
9.64
982.4
24764

.580

.810

.820

.800

.780

.760

$\begin{array}{rr}4.150 & 16.227 \\ 5.450 & 9.000 \\ 3.47 & 185.00 \\ 3.79 & 153.64 \\ 4054.0 & 658.3\end{array}$
$40540 .-108816$

6213.
8.

8.860
1.301
69.37
38.93
1014.2
-162034.

15.579
8.000
25.00
20.19
1729.0
-42130.

7.922 1.423 12.38 7.10 $1729.0 \quad 1368.9$ -36736 . -10489 .

\begin{tabular}{|c|c|c|c|c|c|c|}
\hline $\begin{array}{l}\text { EFFECT I VENESS } \\
\text { NTU } \\
A U(K W / K)\end{array}$ & $\begin{array}{r}H \times 1 \\
.976 \\
28.43 \\
607.09\end{array}$ & $\begin{array}{r}H \times 2 \\
.942 \\
8.71 \\
161.00\end{array}$ & $\begin{array}{r}\mathrm{HX3.1} \\
.978 \\
29.05 \\
523.50\end{array}$ & $\begin{array}{r}H \times 3.2 \\
.978 \\
28.69 \\
95.93\end{array}$ & $\begin{array}{r}\text { HEAT } \\
\text { HX4 } \\
.964 \\
12.63 \\
238.09\end{array}$ & $\begin{array}{c}\text { EXCHANGER } \\
\text { HX5 } \\
.939 \\
10.23 \\
165.66\end{array}$ \\
\hline \multicolumn{7}{|c|}{ LOAD SUMMARY } \\
\hline $\begin{array}{l}\text { FLOW RATE- } \\
\text { TEMPERATU } \\
\text { PRESSURE- } \\
\text { DENS ITY-GI } \\
\text { ENTHALPY - }\end{array}$ & $\begin{array}{l}\mathrm{GM} / \mathrm{SEC} \\
\mathrm{E}-\mathrm{K} \\
\mathrm{TM} \\
/ \mathrm{CC} \\
/ \mathrm{GM}\end{array}$ & $\begin{array}{r}P \\
\text { SU } \\
415\end{array}$ & $\begin{array}{l}\text { PIMARY I } \\
\text { OPLY } \\
3.98 \\
2.59 \\
5.35 \\
154 \\
7.32\end{array}$ & $\begin{array}{r}\text { LOAD } \\
\text { RETURN } \\
4053.98 \\
4.19 \\
4.20 \\
.139 \\
10.63\end{array}$ & \multicolumn{2}{|c|}{\begin{tabular}{rr}
\multicolumn{2}{r}{ SECONDARY LOAD } \\
SUPPLY & RETURN \\
355.91 & 355.91 \\
40.00 & 69.35 \\
15.67 & 9.67 \\
.01842 & .00668 \\
222.00 & 376.53
\end{tabular}} \\
\hline
\end{tabular}


FLUID PROPERTIES AND FLOW RATES

PRESSURE (ATM), TEMPERATURE(K), ENTHALPY (J/GM) AND FLOW RATE (GM/SEC)

$\begin{array}{rrrrr}\text { POINT } & \text { PRESS. } & \text { TEMP. } & \text { ENTHAL. } & \text { FLOW } \\ 1 & 17.250 & 305.00 & 1604.33 & 4212.15 \\ 3 & 16.400 & 305.00 & 1604.04 & 4212.15 \\ 4 & 16.278 & 185.00 & 980.61 & 4212.15 \\ 5 & 16.278 & 185.00 & 980.61 & 3553.88 \\ 6 & 16.181 & 153.64 & \mathbf{8 1 7 . 5 2} & 3553.88\end{array}$

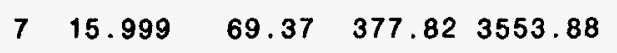

$\begin{array}{lllll}9 & 15.676 & 40.00 & 222.00 & 3553.88\end{array}$

$\begin{array}{lllll}10 & 15.666 & 40.00 & 222.00 & 3197.97\end{array}$

$\begin{array}{llllll}11 & 15.636 & 25.00 & 139.41 & 3197.97\end{array}$

$\begin{array}{llllll}12 & 15.636 & 25.00 & 139.41 & 1468.94\end{array}$

$\begin{array}{lllll}13 & 15.606 & 20.19 & 111.54 & 1468.94\end{array}$
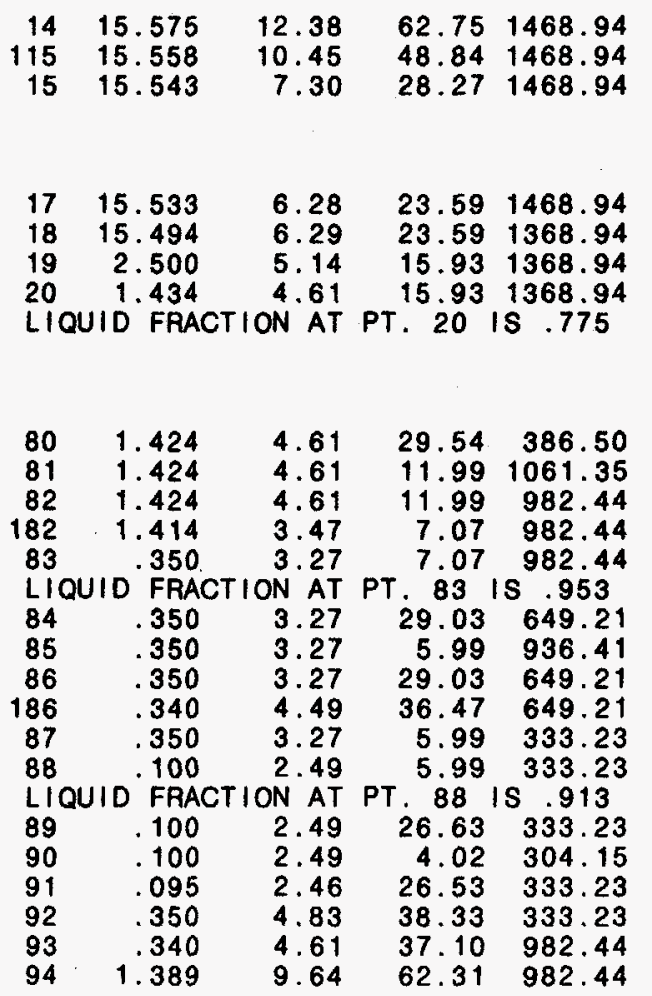

$\begin{array}{rrrrr}\text { POINT } & \text { PRESS. } & \text { TEMP. } & \text { ENTHAL. } & \text { FLOW } \\ 21 & 1.050 & 302.00 & 1583.34 & 4212.15 \\ 23 & 1.100 & 302.00 & 1583.34 & 4112.15 \\ 24 & 1.139 & 178.99 & 944.52 & 4112.15 \\ & & & & \\ 26 & 1.166 & 151.69 & 802.78 & 4112.15 \\ 126 & 1.166 & 151.69 & 802.78 & 3468.61 \\ 127 & 1.243 & 64.90 & 351.89 & 3468.61 \\ 226 & 1.166 & 151.69 & 802.78 & 643.54 \\ 227 & 1.243 & 64.90 & 351.89 & 643.54 \\ 27 & 1.243 & 64.90 & 351.89 & 4112.15 \\ & & & & \\ 29 & 1.294 & 38.93 & 216.76 & 4112.15 \\ 30 & 1.306 & 38.93 & 216.76 & 3097.97 \\ 31 & 1.318 & 22.60 & 131.43 & 3097.97 \\ & & & & \\ 33 & 1.330 & 20.06 & 118.08 & 3097.97 \\ 133 & 1.330 & 20.06 & 118.08 & 1468.38 \\ 134 & 1.348 & 10.87 & 69.07 & 1468.38 \\ 233 & 1.330 & 20.06 & 118.08 & 1629.58 \\ 234 & 1.348 & 10.87 & 69.07 & 1629.58 \\ 34 & 1.348 & 10.87 & 69.07 & 3097.97 \\ & & & & \\ 35 & 1.389 & 9.64 & 62.31 & 3097.97 \\ 135 & 1.389 & 9.64 & 62.31 & 2115.53 \\ 136 & 1.404 & 7.10 & 47.78 & 2115.53 \\ 36 & 1.404 & 7.10 & 47.78 & 386.50 \\ 37 & 1.411 & 4.60 & 29.54 & \mathbf{3 8 6 . 5 0}\end{array}$

$\begin{array}{rrrrr}100 & 4.550 & 3.80 & 9.57 & 4147.98 \\ 101 & 4.550 & 3.99 & 10.13 & 4103.98 \\ 102 & 4.550 & 3.99 & 10.13 & 4053.98 \\ 103 & 4.200 & 4.19 & 10.63 & 4053.98 \\ 104 & 4.200 & 4.19 & 10.63 & 4053.98 \\ 105 & 4.150 & 3.47 & 8.47 & 4053.98 \\ 106 & 5.450 & 3.79 & 10.01 & 4053.98 \\ 107 & 5.450 & 3.82 & 10.08 & 4153.98 \\ 108 & 5.400 & 3.37 & 8.91 & 4153.98 \\ 109 & 5.350 & 2.59 & 7.32 & 4153.98 \\ 110 & 5.000 & 2.90 & 7.68 & 4153.98\end{array}$

POINT PRESS. TEMP. ENTHAL. FLOW $\underset{\sim}{\square}$

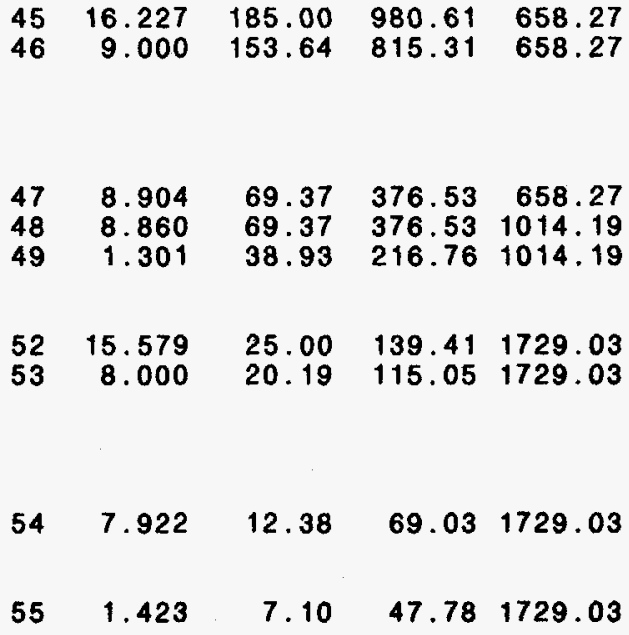


case. Electric power required during the cooldown is $12 \mathrm{MW}$. Electric power costs per cooldown would be about $\$ 25 \mathrm{~K}$ for magnet $\mathrm{A}, \$ 9 \mathrm{~K}$ for $\mathrm{B}$ and $\$ 11 \mathrm{~K}$ for $\mathrm{C}$. The RHIC refrigerator could be used to cool the Long String if the R\&D refrigerator were to fail for some reason or if the load proves unexpectedly high. Because the refrigerator would operate at only 5 or $10 \%$ of its capacity, the efficiency of the plant would be 10 ( $s 10 \%$ Carnot). The power required to operate the plant under these conditions is estimated to be 1.6 to $2.1 \mathrm{MW}$. If it were desired to operate at low temperature, this refrigerator could cool the Long String below 3K. Table 2 summarizes the expected refrigeration equipment operating conditions for the Long String

TABLE 2

REFRIGERATION EQUIPMENT SCHEDULE

\begin{tabular}{|c|c|c|c|c|c|c|c|}
\hline \multirow[b]{2}{*}{ OPERATION } & \multicolumn{3}{|c|}{ R\&D Refrigerator } & \multirow{2}{*}{$\frac{\text { RHIC }}{\text { ON/OFF }}$} & \multicolumn{3}{|c|}{ Cost Factors } \\
\hline & $\begin{array}{l}\text { No. RM. } \\
\text { TEMP. } \\
\text { COMPR. }\end{array}$ & $\begin{array}{l}\text { COLD. } \\
\text { CIRC. } \\
\text { COMPR. }\end{array}$ & $\begin{array}{l}\text { COLD. } \\
\text { VAC. } \\
\text { PUMP. }\end{array}$ & & $\begin{array}{l}\text { ELEC. } \\
\text { POWER } \\
\text { (MW) }\end{array}$ & $\begin{array}{r}I \\
R \\
(\mathrm{~g} / \mathrm{s})^{2}\end{array}$ & $\begin{array}{l}1 \mathrm{D} \\
1 / \mathrm{hr} .)\end{array}$ \\
\hline Cooldown & 0 & off & off & on & 12 & 0 & 0 \\
\hline Steady State & 2 & on & off & off & 0.36 & 10 & 50 \\
\hline Quench Recovery & 3 & on & off & of $f$ & 0.54 & 12.5 & 60 \\
\hline Low Temp. Test & 3 & on & on & off & 0.54 & 12.5 & 60 \\
\hline Warm-up & 3 & off & off & off & 0.54 & 0 & 0 \\
\hline
\end{tabular}

Power Supply

BNL has three power supplies available for use in the STF. Each power supply is capable of delivering $5000 \mathrm{~A}$ rms (7500 A peak) at 50 volts maximum. These units can be connected in series or parallel as needed to form power systems for the inital test program. Use of these units will permit delaying pro- 
curement of the final magnet string power system until the choice of magnet type has been made. At that time all the power requirements will be known and the proper procurement can be made.

Limitations and capabilities of the interim system as arranged for each of the three magnet types are as follows:

Magnet Type A\& B :

Two power supply units will be operated in parallel. This will make possible full operations of all tests up to nine magnets for type A or seven magnets for type $B$. With longer strings the ramp rate would have to be reduced proportionally because of insufficient voltage. No other test limitation exists. These units would be placed in the East Injection Hall, near the R\&D refrigerator, where they operated previously for the CBA Fu11 Ce11 Test. Primary power, cable tray and cable are already installed in this position. Magnet Type C:

Two of the existing power units operating in parallel would supply the 12000 A circuit and the third unit the 6000 A circuit. Current would be 1imited to $5000 \mathrm{~A}$ rms for each supply. This would permit all tests except a d.c. run beyond $10000 \mathrm{~A}$. By using low resistance warm cable, the cable voltage drops would be reduced to less than 15 volts. This would permit sufficient voltage for ramping of the full magnet string as specified (200 sec. cycle).

Once the magnet type has been chosen, the final power system can be engineered and procured. A preliminary estimate of the items required is shown in the circuit sketches in Fig. 16. Their costs are included in the cost estimate. For magnet types $A$ and $B$ the principal delayed procurement item is the ramping power supply capable of ramping the Long String to SSC operating current and back to zero in $15 \mathrm{~min}$. (220V a $4200 \mathrm{~A} \mathrm{rms}, 7500 \mathrm{~A}$ peak). Most of the 
D.C.

INTERRUPTER

$2000 \mathrm{VDC}$

AT $7.5 \mathrm{KA}$

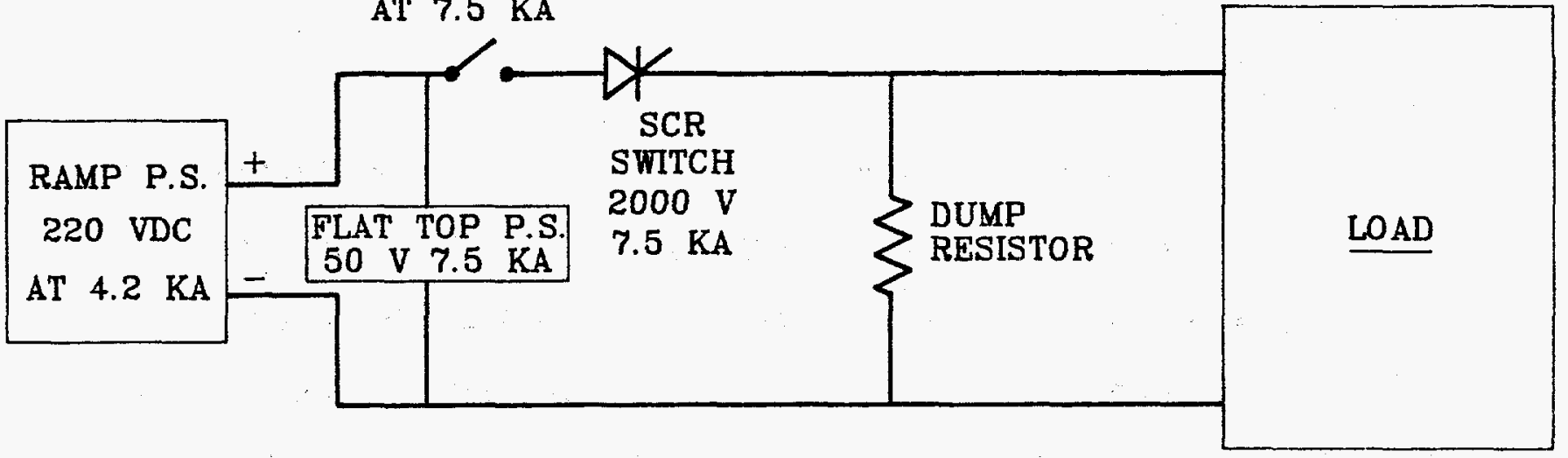

WIRING DIAGRAM FOR MAGNET A OR B

SCR SWTTCH

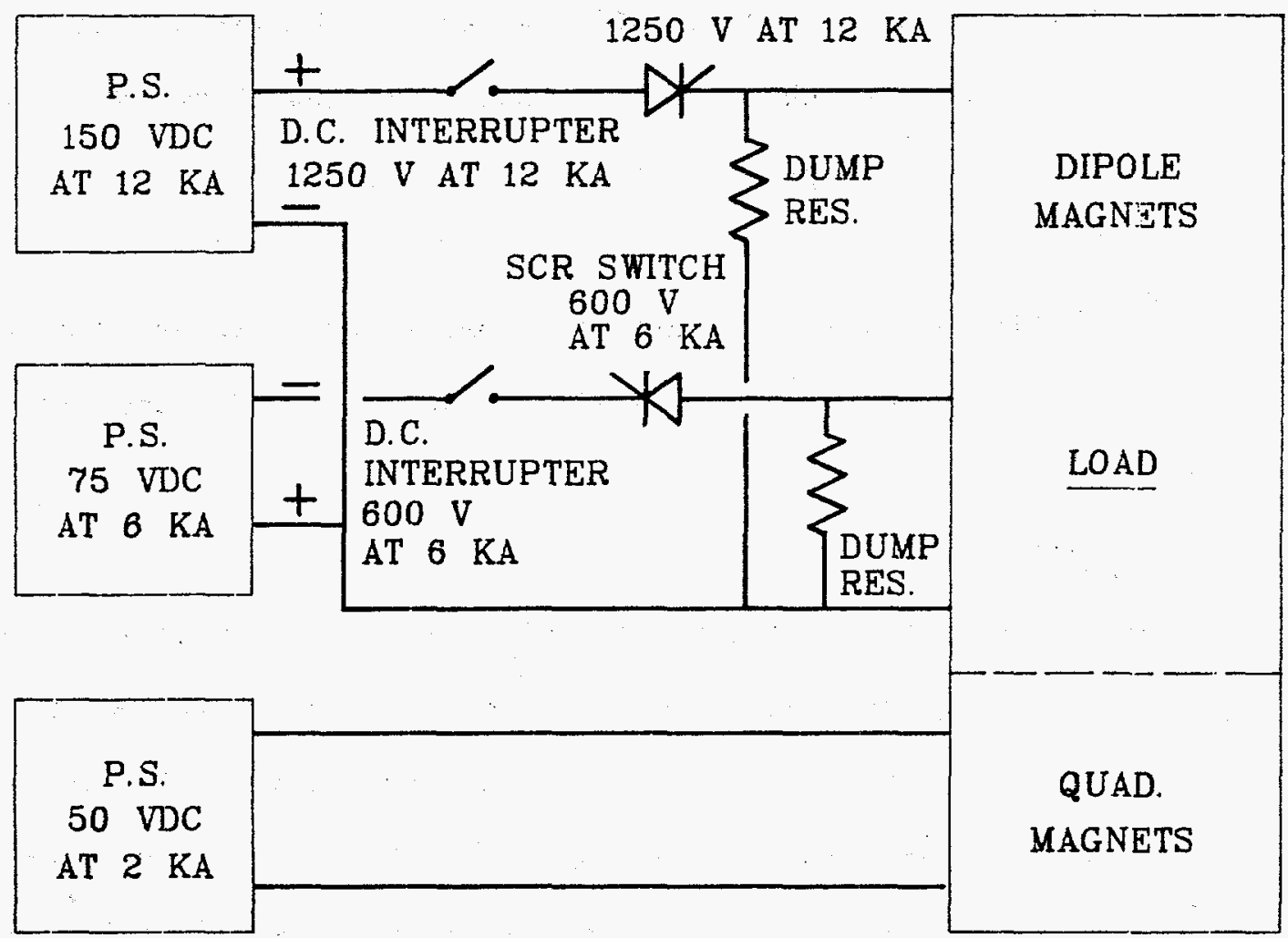

WIRING DIAGRAM FOR MAGNET C

POWER SUPPLY AND QUENCH PROTECTION DIAGRAMS FOR LONG STRING TESTS

Figure 16 
other items shown can be procured as soon as funds are available for the STF. For magnet type $\mathrm{C}$ there are 4 power supplies that must be procured. Quench Protection

Magnet Type A:

Quench protection for the type A magnet requires heater firing circuits to quench all magnets in a group and a system to extract the energy from the nonquenching magnets. The heater firing circuits will be similar to those developed at FNAL. Energy extraction will be accomplished by SCR switches backed up by a mechanical d.c. interrupter. Similar energy extraction systems are in operation at both BNL and FNAL.

Magnet Type B:

The system required for magnet type $B$ is identical to that described for magnet type A except that an additional SCR current shunting system is required. This additional requirement has not been studied in detail by BNL engineers and the cost shown is only a rough estimate.

Magnet Type C:

No heater firing or special current shunting circuits are required. Quench protection is provided by the SCR switches, the D.C. interrupters and the dump resistors.

Quench Detection

There are many ways to detect the initiation of a quench, but reliability must be the overriding consideration. The design of reliable electronics is a debatable subject and long standing divergent views exist. The system favored at BNL is based on simplicity and redundancy. This system detects and compares the voltage appearing across magnet blocks with each other and with the expected di/dt value. This detection and comparison is performed by at least two indepen- 
dent sets of electronics and is accomplished locally in analog form. A difference voltage exceeding a settable threshold results in a quench signal being sent to the appropriate quench protection system, the power supply and the controlling computer complex.

\section{Control System}

A minimum of hardware and software development will be necessary to implement a sound but flexible control system for the SSC System Test Facility. A commercial process control computer system purchased for the CBA would only require the reconfiguration of some of its input-output hardware and the provision of additonal software to meet STF needs.

A block diagram of the control system is shown in Figure 17. At the top is a DEC PDP $11 / 44$ host processor located in a local cryogenic operations area. Peripherals include alarm and logging typewriters, program development CRT's and three color graphic operator interface displays. The host provides drive capability for the operator's consoles and performs communications, polling, data storage and retrieval duties. The cryogenic refrigerator and its compressors are monitored and controlled by the redundant PDP 11/34 processors indicated. Accurate temperature data acquisition requires the equivalent of 18 bit analog conversion and is performed by in-house designed and constructed distributed microprocessors. This system is tailored to interface to all types of cryogenic temperature sensors produced by our calibration facility and converts the signal to linearized engineering units. Connection to the process system is either through serial link or by applying the data directly to the processor's unibus. Monitoring and control of magnet, cryogenic, vacuum, power supply etc. functions would be accomplished by the two PDP $11 / 23$ distributed microprocessors shown. Each station has a fully redundant hot backup processor and memory for 


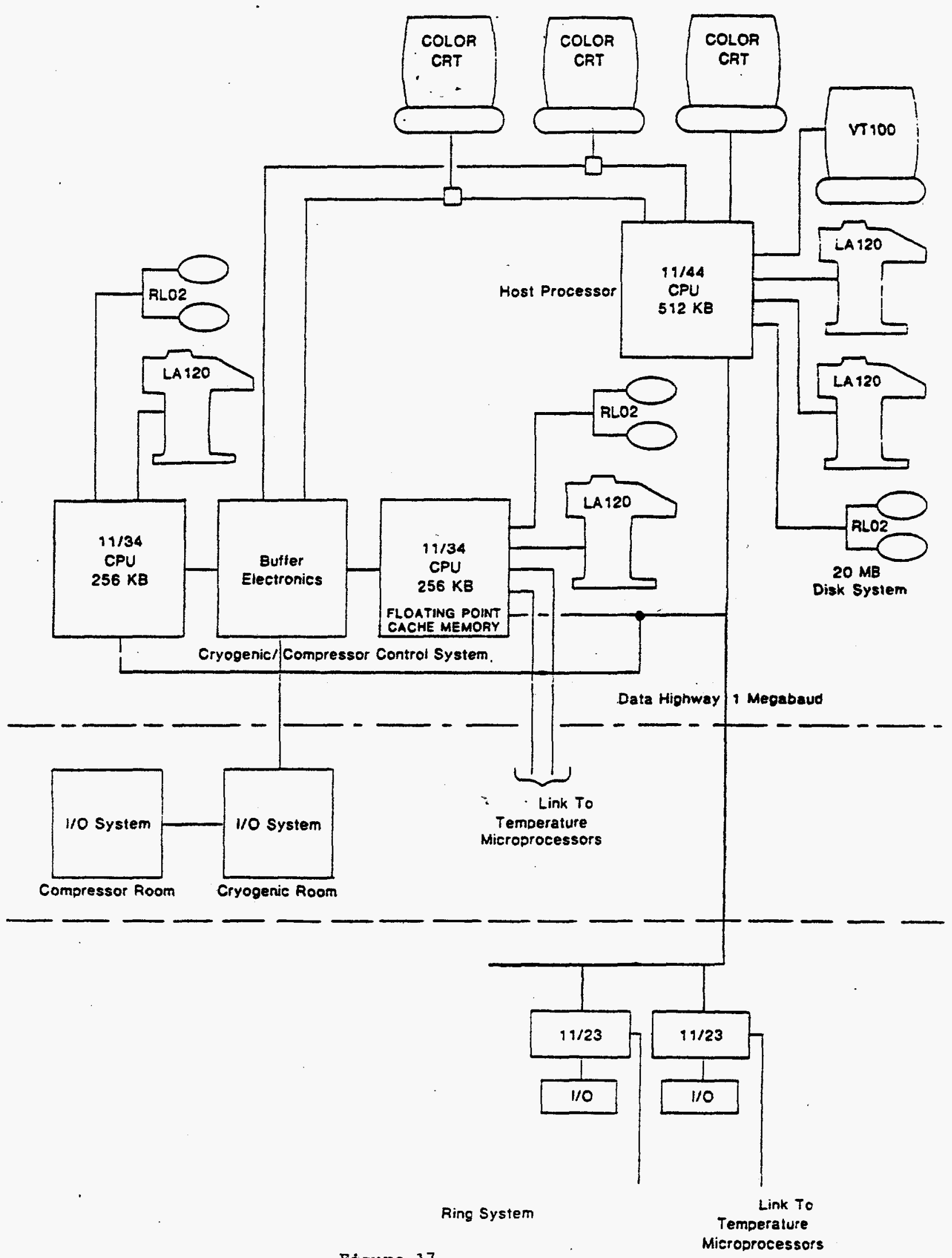

Figure 17

Cryogenic Control System Architecture 
reliability, plus input and output hardware to interface analog and digital signals. Initially these satellites are down loaded from the host to install an operating system, control logic and communications programs, so they can act as stand alone control stations. The distributed microprocessors are polled by the host via a bidirectional one megabaud data highway. Images of a11 system data bases reside in the host which permits real time process interaction and color graphic display at the operator's console. Magnet temperature data acquisition would utilize the same distributed microprocessor hardware, but in this case would be interfaced to the $11 / 23^{\prime}$ s. Power supplies are driven by an internal microprocessor controller. Interfacing to the process system would be paralle1 digital in nature enabling on/off and ramp rate control.

The extension of the commercial process control computer system to the System Test Facility is a minor extrapolation of its demonstrated capability. Smaller but similar systems have been successfully used to monitor and control magnets through programmed cooldown and warmup cycles, measure heat loads and vacua, control magnet lead flows, provide alarm and interlock systems and totally automate multiple screw compressor installations. Where normal 12 bit ana$\log$ conversion does not provide enough resolution a 16 bit converter can be interfaced to digital input channels or read by an 18 bit temperature channel.

The PDP $11 / 44$ host processor has FORTRAN 77 installed and can run logging and simulation programs by reading or writing to any of the system data bases. However, since local mass storage consists of only two 10 megabyte RL02 discs, it is planned to install DECNET software to enable high speed transfer of data from the $11 / 44$ to the On-Line Data Facility. OLDF consists of a network of VAX computers which could be used to run simulation and data reduction programs. This option is beneficial since it would unload the process host, and enable the 
user to upgrade to a VAX and have approximately 3 gigabytes of mass storage at his disposal.

Enlarging the scope of the process control system to include STF magnets is a straight-forward step. Software development, including the DECNET option, should require only two to three man-years of time. Hardware development would be minimal consisting of special interface circuits and additional I/O to meet STF needs. In most cases STF control and instrumentation would consist of the implementation of previously tested and proven solutions.

\section{Data Acquisition}

A rough estimate of the number and type of data points necessary to support the System Test Facility is listed below. Estimates are based upon a Magnet type A string of approximately 70 magnets with recoolers spaced every half ce11. Two main and six correction supplies are assumed to power the magnet string along with the ramp control, quench detection and energy extraction systems.

Cryogenic temperature sensors utilized for cooldown monitoring and heat load measurements would include diode-thermistor pairs and redundant germanium thermometers mounted at the inlet and outlet of the recoolers. Conventional pressure transducers would measure overall magnet pressure drop, and supply and return header pressures. Flow measurement will be done by conventional mass flow-corrected venturi meters where applicable, plus cryogenic ultra-sonic mass flow meters to measure low mass flows without the penalty of significant pressure drop. Such devices have been developed at BNL and would be extremely useful in measuring recooler return flows. This would provide a second and completely independent method of measuring magnet string heat loads. Recooler level will be controlled by process computer DDC using superconducting level 
gauges as the process input. Magnet lead flow would utilize direct reading mass flow meters with either local supervisory or DDC control loops. Lead voltage drops will be measured and used as process inputs for lead thermal protection and alarm systems.

Hardware for the vacuum system consists of turbo pump assemblies for insulating vacuum and ion pumps for beam pipe ultra high vacuum. Insulating vacuum gauging would be installed on each magnet with one vacuum chassis readout (thermocouple/cold cathode) per half cell. Ion pump distribution would be on a per magnet basis. Each vacuum chassis and al1 ion pump currents will be monitored by the process computer system.

Two main power supplies and six correction supplies will be used to energize the BNL magnet string. Ramp control parameters would be preloaded into power supply microprocessor controllers by the process computer system. A global clock would synchronize the ramp of the various power supplies and be used as a time marker for fast data acquisition.

Instrumentation for magnet protection and data acquisition is dynamic, requiring reasonably fast sampling and local buffering. A portion of the required hardware is commercially available but most has been developed in-house to meet the unique requirements of superconducting magnet systems. A quench detection system will trigger heater firing units to remove stored energy safely via an energy extraction system. Consistent with long standing BNL philosophy, any interlock system that could cause physical damage upon failure may be computer controlled but will be backed up by hard wired logic.

Point Summary:

Cryogenic thermometers (diodes, thermisters, germanium) 100 ea. Pressure transducers 6 ea. 
Flowmeters

Recooler level gauges

Lead mass flow

Lead voltage drop/bypass

Insulating vacuum gauges,

(thermocouple, cold cathode 70 ea.)

Insulating vacuum chassis, (24 ea., 2 gauges)

Ion pumps/current monitor

Main Magnet power supplies/voltage

Correction power supplies/voltage

Current transducers

Quench recorders (channels)

Quench detectors

Quench propagation heaters

Energy extraction system
6 ea.

13 ea

12 ea.

12 ea.

140 ea.

48 ea.

70 ea.

2 ea.

6 ea.

8 ea.

150 ea.

$140 \mathrm{ea}$.

140 ea.

1 ea.

Instrumentation defined in the above list will vary depending upon the type of magnet chosen for the SSC and taste. The list is sufficient as a first pass to outline the magnitude of the data acquisition and control tasks but more definition will be necessary before actual design can start.

Additional process computer $\mathrm{I} / 0$ will have to be purchased or constructed in-house to match the instrumentation point list for the STF. Ample room is available in the existing cabinets for expansion so reconfiguration will only mean the installation of additional I/O cards and buckets. Meeting the software requirements for cryogenic, vacuum and power supply control and data acquisition requires a redeclaration of $I / O$ points and enlarging some existing applications routines, plus writing a few new ones. 
Dynamic data acquisition will be new to the process system but can be accomplished in a number of ways. Microprocessor front ends can be constructed to interface to the quench recording system and communicate with the host via the existing 1 megabaud data highway. Data highway to multibus interface cards are commercially available. A CAMAC system could be interfaced directly to the PDP11/44 host by using equipment and software that are readily available at BNL. Another possible option is to adopt a portion of the FNAL system for our use. Cost estimates for the additional interface hardware are included in the cost summary.

In order to reach a state of agreement among interested parties, BNL intends to form an STF Data Acquisition Committee composed of people from BNL, FNAL and other labs. The group will identify all parameters to be measured in the string test and quantify required resolution, sampling speeds and interfaces for dynamic data points. 
D-36

SHORT STRING TEST

The MAGCOOL test facility in Building 902 is proposed as the site for the Short String Test. MAGCOOL was designed and used to test CBA magnets prior to their installation in the tunne1. It uses a Model 4000 refrigerator built by CTI (now Koch). This refrigerator has a nominal capacity of $1580 \mathrm{~W}$ at $4.5 \mathrm{R}$. A temperature-entropy plot of the cycle is shown in Figure 18. This site permits the use of the magnetic field measurement and data acquisition system regularly used in the magnet test facility.

The requirements for this test program are given in Section III of the Task Force report. The major portions of the test from the standpoint of time required are (1) the 100 thermal cycles ( 80 from $300 \mathrm{~K}$ to $80 \mathrm{~K}$ and back, the remaining 20 from $300 \mathrm{~K}$ to $4 \mathrm{~K}$ and back), (2) the 500 quenches and the time required to recover and (3) the approximately $10^{5}$ simulated SSC ramps. For the purpose of this estimate it has been assumed that the test will be divided into 10 sections. Each section would consist of the steps listed below. The time (assuming Magnet A which has the greatest cooldown weight) estimated for these tests is also given below.
1. 9 thermal cycles from $300 \mathrm{~K}$ to $80 \mathrm{~K}$ and back
(c) $48 \mathrm{hr} .=432 \mathrm{hr}$.
2. Cooldown to $4 \mathrm{~K}$
3. Magnetic measurements
4. $10^{4}$ ramping cycles at $200 \mathrm{sec} /$ cycle
5. 50 quenches followed by recovery
(c) $2 \mathrm{hr} .=100$
6. Magnetic measurements
24
7. Warm-up to $300 \mathrm{~K}$ completing 10 th thermal cycle 


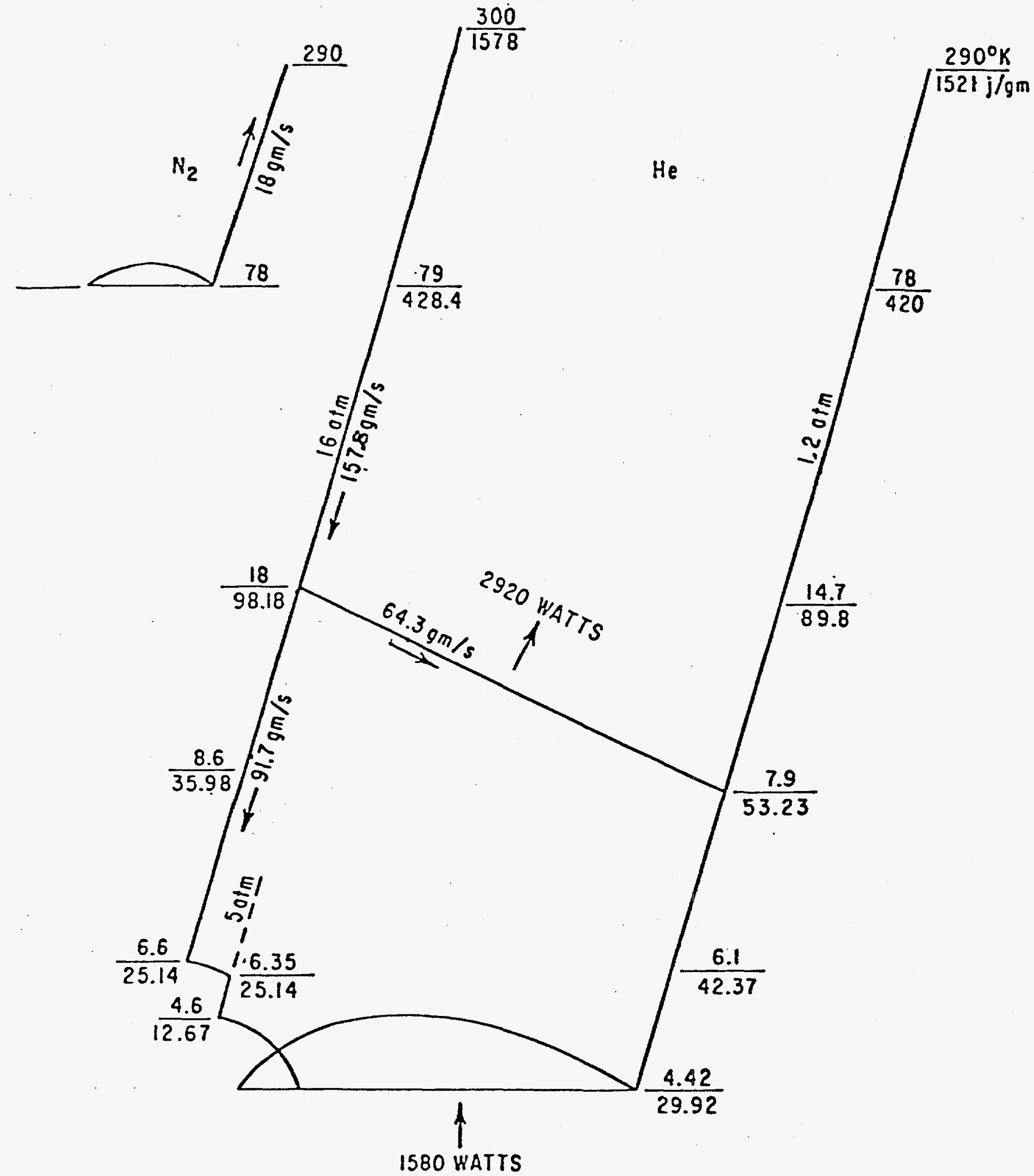

Figure 18

MAGCOOL T-S DIAGRAM MODEL 4000 BNL 
The Short String Test will overlap the period of time that the test and measurement of magnets produced for use in the Long String Test is anticipated if Magnet Type $\mathrm{A}$ is chosen. In order to avoid any delay in completion of the Short String Test, MAGCOOL would be used exclusively for that test until it is finished. The PAT refrigerator, which is also located in Building 902, will be used to provide cooling for the test and measurement of individual magnets.

As this test set-up will be in place for a relatively long period of time ( $>16$ months), it is desirable to put it in a location which will offer little obstruction to the flow of magnets in this assembly area. For magnet types $A \& B$, the solution chosen is to place the Short String in two large trenches in the floor which were used for utilities when this building was the Experimental Area for the Cosmotron. The trenches and their relation to MAGCOOL are shown in Figure 19. The two trenches run almost the length of the building and a cross trench at the end furthest from MAGCOOL makes it easy to arrange the magnets in a "U". The dimensions of the $171 \mathrm{ft}$. long trenches, $6 \mathrm{ft}$. wide $x 8 \mathrm{ft}$. high and $7 \mathrm{ft}$. wide by $8 \mathrm{ft}$. high respectively, are more than adequate for installation of the magnets. The heavy reinforced concrete covers over the trenches would be removed to permit installation of the magnets with the building crane (40 $\mathrm{T}$. capacity). When the cover plates are replaced, the magnets would be protected against any accidents due to floor activity. Easy access to the trenches is permitted by stairways at each end of the building. Access control for safety reasons will be easily accomplished. For magnet type $C$ the half-cell cannot be bent in the middle, and it would be placed in B1dg. 905 (adjoining B1dg. 902) at normal floor level.

Two papers which describe MAGCOOL are attached to this report. The major cryogenic items of equipment available in this system are as follows. 


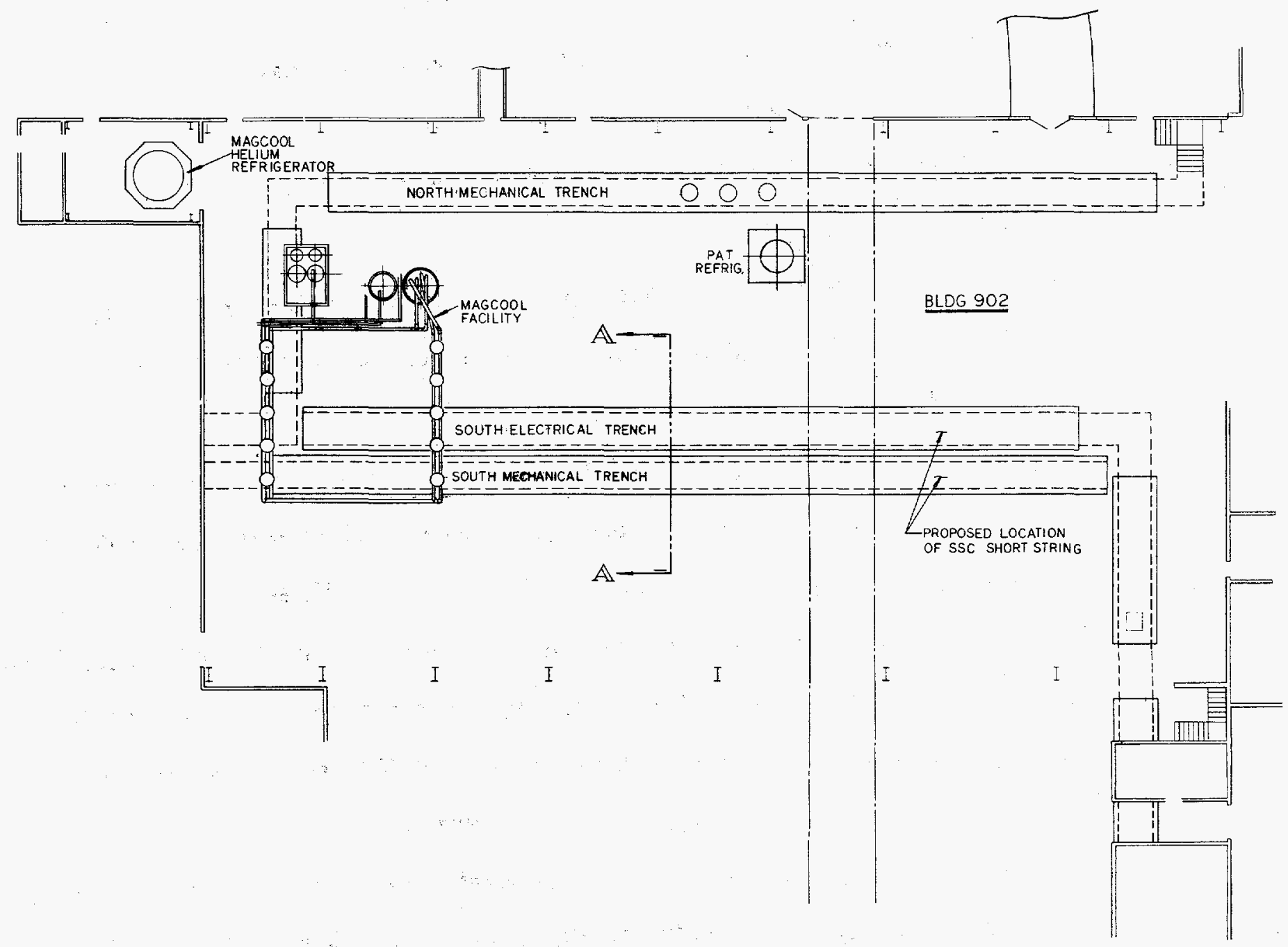

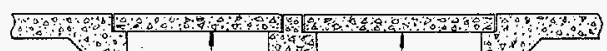

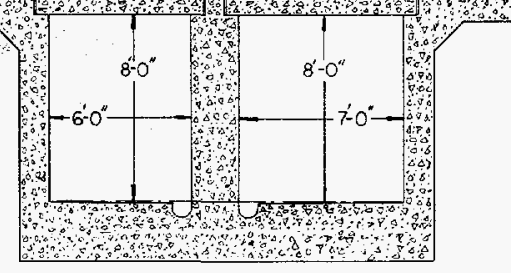

$\frac{\text { SECTION A A }}{\text { SCALE } \frac{3}{8}=1-0^{*}}$

Figure 19: Floor Plan of Bldg. 902 showing proposed location of Short String. 
3. Three $100 \mathrm{HP}$ Sullair Screw Compressors for circulation of helium during (1) purge, (2) cooldown and (3) warm-up

4. Dual cryogenic purifier for system clean-up and make-up

5. 40,000 liter liquid nitrogen storage vessel

6. Special heat exchangers for cooldown and warm-up

7. Heaters for warm-up

8. Liquid-to-gas heat exchangers (precooler and subcooler) with ejector and provisions for cold circulating and/or vacuum compressors.

9. Process control computer

This equipment can be configured to suit the particular requirements of the magnet type chosen for SSC. The only major item of equipment which would have to be added is a helium dewar which would serve as a buffer tank during quenches. The addition of the tank would permit holding the low temperature gas ( $T$ SOK) which is evolved from the magnets during quench until it is used for make-up when the magnets are recooled. A dewar of 5000 iter capacity and pressure rating of $20 \mathrm{~atm}$ would serve well for this application.

The MAGCOOL refrigerator has operated reliably for 8 years and will be capable of meeting the needs of the Short String Test. The available capacity from the MAGCOOL system at each point of operation is summarized below.

$\begin{array}{lc}\text { Refrigeration } & 1580 \mathrm{~W} \text { at } 4.5 \mathrm{~K} \\ \text { Liquefier } & 7.5 \mathrm{~g} / \mathrm{s}(2251 \text { iters/hr }) \\ \text { Cooldown } & 80 \mathrm{~g} / \mathrm{s} \text { at } \sqrt{ } 80 \mathrm{~K}\end{array}$


Utilizing BNL's MAGCOOL facility for the SSC Short String Test thermal cycle program requires no additional control hardware. All necessary instrumentation is available with ample process computer I/O. A stand-alone microprocessor temperature acquisition system has been operating for the past few years and is presently interfaced to platinum, diode and germanium sensors. Spare temperature channels are available to measure process fluid temperatures along the short string for heat load calculation purposes. Existing lead flow and voltage drop alarm and interlock systems would function as designed for CBA magnet testing.

Process control system applications software already provides for automatic thermal cycling of magnets from room to $\mathrm{N}_{2}$ temperature and warmup. (A similar cycling test was performed on a CBA magnet which ran 24 hours per day, unattended from 1600 to $0800 \mathrm{hrs.}$ ) Cooldown to helium temperature and magnet excitation follows. Cryogenic system upsets caused by quenches are easily tolerated by the system. Special routines are triggered by quench detectors which change the control philosophy so that excess gas is not lost but stored in a thermal buffer tank and then used to maximize the magnets' cooldown rate into their next cycle.

The magnetic field of the half-cell of magnets will be measured by a field measurement probe (Fig. 20) being developed to measure $16 \mathrm{~m}$ magnets. This probe is designed to be pulled through a long magnet or magnets, stopping to measure the field along the way, and communicating with the outside world via a tether containing wires and gas lines. Since it is designed to operate in a warm environment, an insulating warm bore tube would be permanently installed in the magnets to allow access to the aperture. Since this tube is expected to have a heat load of 5-10 W/magnet, it would be used only when measurements are being 


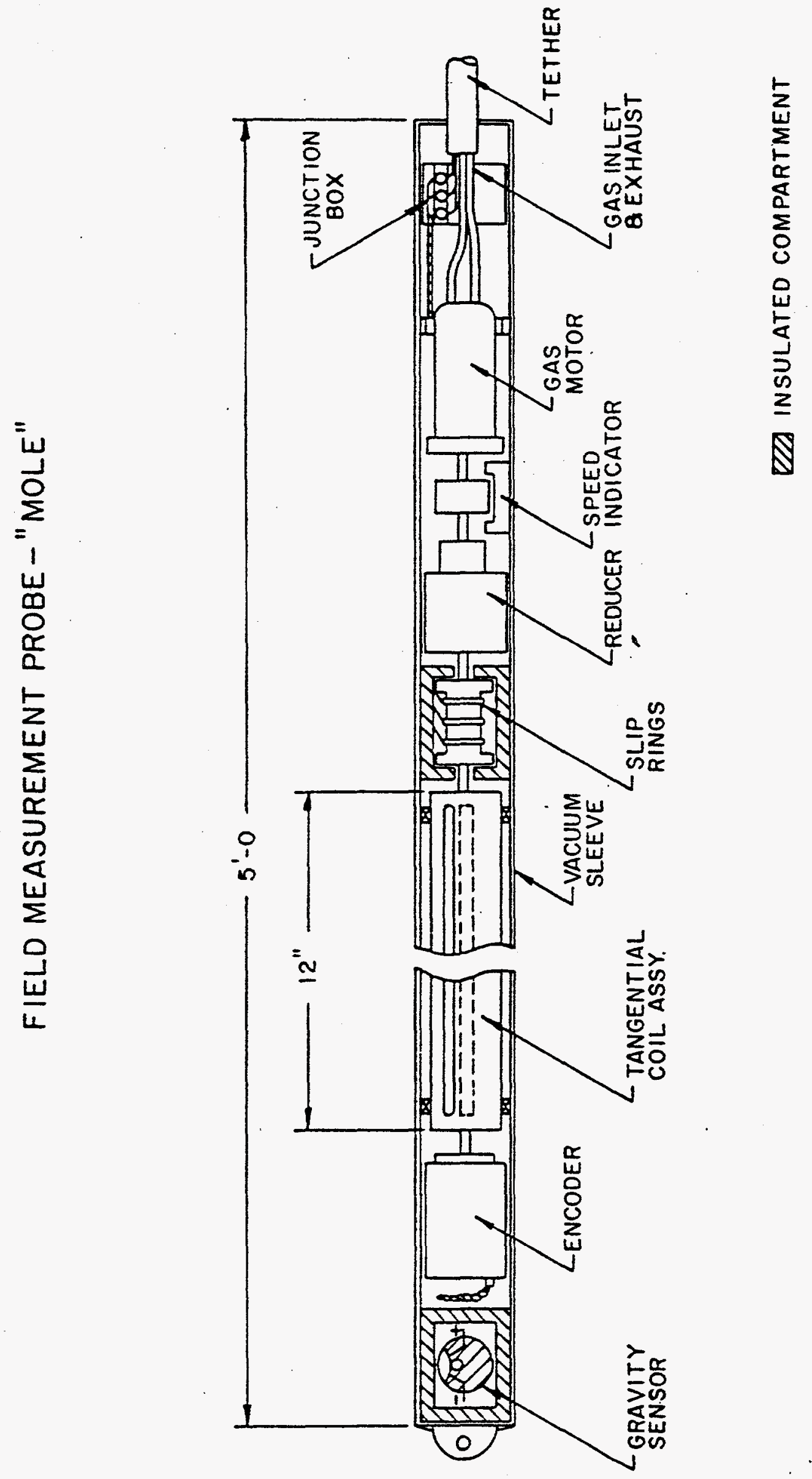

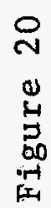


made; otherwise it would be evacuated and thus would add little to the heat load during most testing.

One of the three existing $5000 \mathrm{~A} \mathrm{rms}$, $50 \mathrm{~V}$ power supplies would be used for magnets $A$ or $B$ and would satisfy all requirements. Short string testing for magnet $C$ could not begin until the new supplies were available, but since this would be by the end of FY86, no delay is foreseen. The circuits would be as shown in Fig. 21 .

The quench protection and detection systems used here will be exactly as described above for the Long String. 
D-44

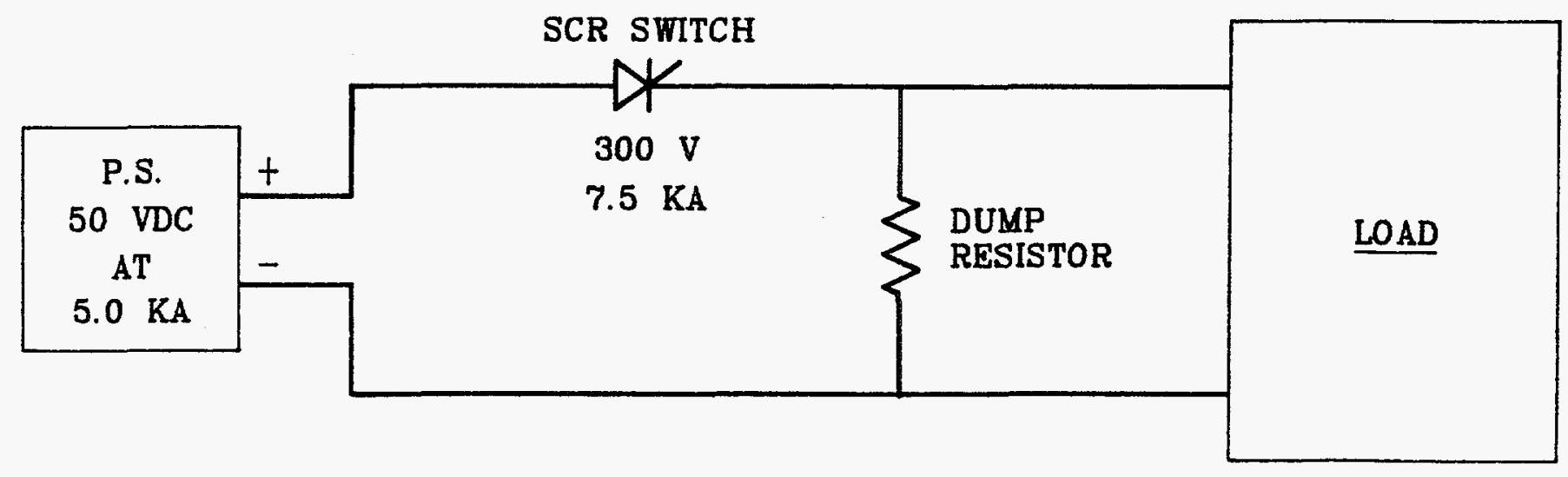

WIRING DIAGRAM FOR MAGNET A OR B

SCR SWITCH

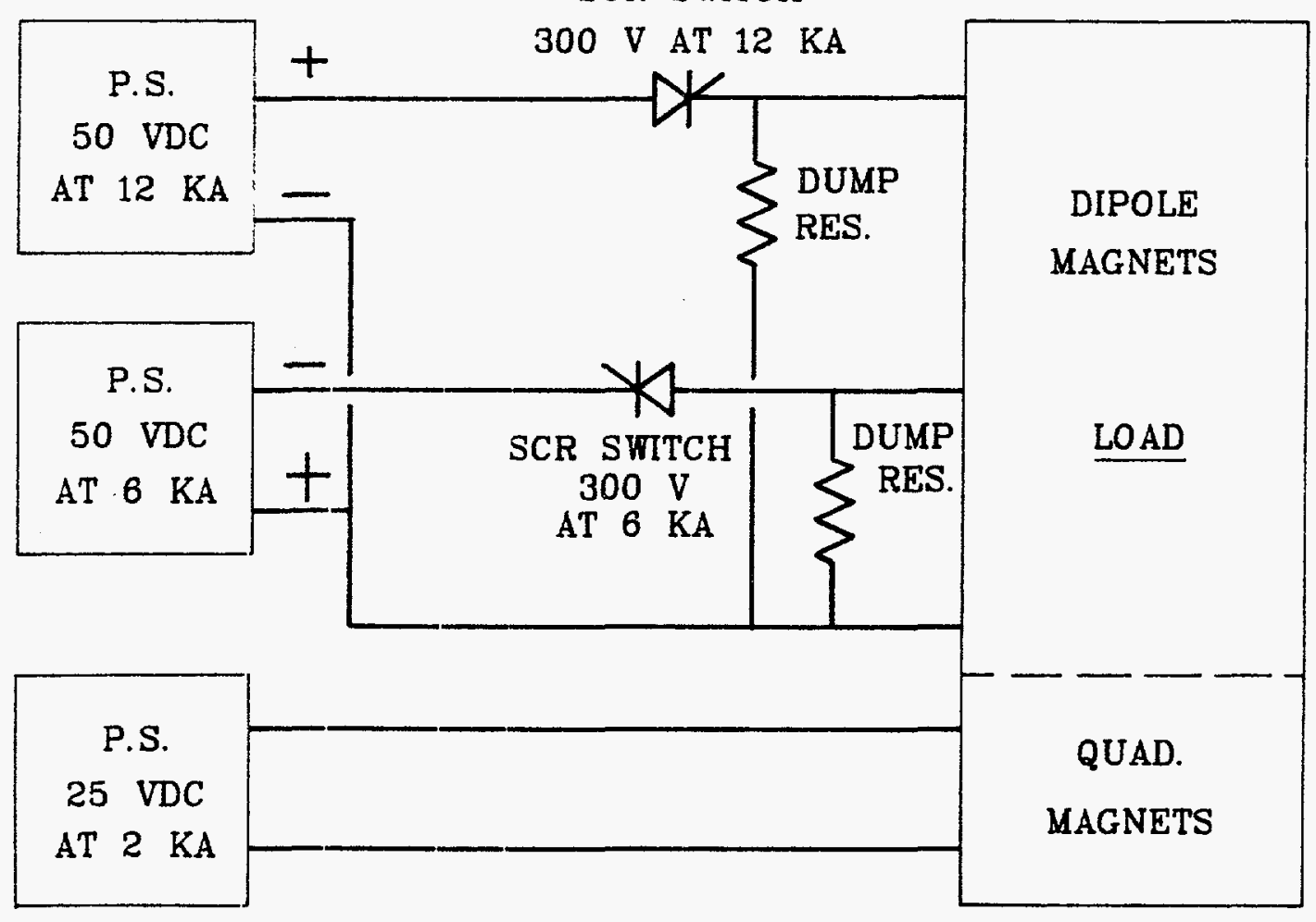

WIRING DIAGRAM FOR MAGNET C

POWER SUPPLY AND QUENCH PROTECTION DIAGRAMS FOR SHORT STRING TESTS 
Presented at the 1981 Cryogenic Engineering Conference, August 10-14, 1981 San Diego, California

"MAGCOOL" - THE PRODUCTION COOLING FACILITY FOR ISABELLE MAGNETS*

J.A. Bamberger, M. Afrashteh, D.P. Brown,

W.J. Schneider, J.H. Sondericker, R.C. Wu

Accelerator Department

Brookhaven National Laboratory

Upton, New York 11973

\section{INTRODUCTION}

The ISABELIE proton accelerator uses over one thousand superconducting magnets to guide the particle beams in two circular rings, $3.8 \mathrm{~km}$ in circumference. Prior to their installation in the tunnel all magnets must be tested and measured at their $3.8 \mathrm{~K}$ operating temperature. This paper describes the refrigeration system, called "MAGCOOI," to accomplish this task.

\section{SYSTEM FUNCTIONS}

The magnet test procedure involves five main tasks which must be performed in sequence, each with distinct operating requirements for a set period of time. The MAGCOOL system provides five test stands, each of which is capable of performing any of the five basic tasks. The normal duty cycle is 24 hours per task; therefore with one magnet under test at each of the stands the production test rate is five magnets per week for a five day operation. The System Block Diagram, Figure 1, schematically shows the arrangement of the test stands. For illustration, only one of the five tasks is shown for each of the test stands. A system of headers and remotely operable valves allows any of the basic functions to be performed at any of the test stands. A process control computer is used to monitor and control these tasks which are described below.

TWork performed under the auspices of the U.S. Department of Energy. 
PUMP AND PURGE PROCEDURE

In the ISABELLE ring 45 magnets will be cooled in series with supercritical helium passing through the magnet. All contaminants must be removed prior to the magnets' initial cooldown and test in MAGCOOL. The tested magnets must be warmed up to room temperature and delivered in clean, purged condition ready for installation in the ISABELLE ring.

After initial connection to the test stand, successive evacuations to below 50 microns are performed followed by backfill with pure helium. Helium gas is then circulated using forced flow from the screw compressor which circulates helium gas through one of the dual cryogenic purifiers and then through the magnet at the test stand. The purifier is designed for a flow rate of $15 \mathrm{~g} / \mathrm{sec}$ at a pressure of $8 \mathrm{~atm}$. The capacity of the puIifier is based on an assumed contamination level of $50 \mathrm{ppm}$, water and air and a duty cycle of 24 hours per dual bed, including reactivation time. An automatic fill system for the liquid nitrogen cooled charcoal bed is provided. Reactivation of the purifier is by means of nitrogen gas which is warmed by an external electrical heater.

MAGCOOL provides the following equipment for the pump and purge procedure:

- Two-stage mechanical vacuum pumps for evacuation

- Pure helium gas for purging the evacuated system

- Circulating screw compressor system

- Four stage oil removal at the screw compressor outlet

- Dual cryogenic purifiers

\section{COOLDOWN I}

During Cooldown I the magnets are cooled from room temperature to $85 \mathrm{~K}$. This is accomplished with a counterflow heat exchanger followed by a liquid nitrogen cooled plate-fin heat exchanger. The weight of a dipole magnet is about $6500 \mathrm{~kg}$ requiring removal of about 500 megajoules to bring this material, mostly iron and stainless steel, to $85 \mathrm{~K}$. The cooldown rate for this process must be limited in order to avoid excessive stresses which could result from temperature gradients beyond those permitted. The refrigerant and magnet $\Delta T^{\prime}$ s are monitored and the helium flow rate and temperature is automatically regulated to achieve an optimum cooling rate without exceeding allowable temperature gradients. A screw compressor, similar to the "purge compressor" is used to circulate the coolant helium stream through the heat exchanger and magnet systems. 
The maximum available flow rate is 60 g/sec helium with a return pressure of 8 atm and a supply pressure of 12 atm.

Liquid nitrogen is circulated through the magnet heat shield during the Cooldown I operation cooling the aluminum shield to $85 \mathrm{~K}$ and bringing the multi-layer insulation to the equilibrium gradient for this temperature level.

\section{COOLDOWN II}

During this operating phase the magnets are cooled from 85 $\mathrm{K}$ to $6 \mathrm{~K}$, requiring the removal of about 25 megajoules per magnet for this portion of the cooldown. An existing claude cycle helium refrigerator is used for the MAGCOOL operations below $85 \mathrm{~K}$. This refrigerator, with about $1 \mathrm{kw}$ capacity at 4.5 $\mathrm{K}$, has dual expanders, uses liquid nitrogen precooling, and is equipped with oil flooded screw compressors driven by electric motors totaling $550 \mathrm{kw}$.

Figure 2 shows the flow schematic for MAGCOOL operations below $85 \mathrm{k}$. The refrigerator itself is not shown in Fig. 2 , but its supply and return lines are included and identified as cold supply and return."

Heat Exchangers $\mathrm{HX} \mathrm{C} 2 \mathrm{~A}$ and $\mathrm{HX} \mathrm{C} 2 \mathrm{~B}$ are used for Cooldown II. A portion of the cold high pressure return stream from the "Test and Measure" circuit is used in cooldown heat-exchanger HX C2A. This three-pass heat exchanger cools a $15 \mathrm{~m} / \mathrm{sec}$ circulating side-stream from the cooldown compressor which then circulates through the Cooldown II headers to the magnet and then back through the heat exchanger. The lower heat exchanger, $\mathrm{HX} C 2 \mathrm{~B}$, provides additional cooling capacity during the magnet "training" sequence.

When the magnet has reached equilibrium conditions near the end of the Cooldown II procedure, a total heat load determination is made on each magnet by measuring the temperature rise across the magnet with germanium resistance thermometers and determining the flow rate from calibrated flow meters provided in the circulating gas system.

\section{TEST AND MEASURE}

The refrigeration circuit for the Test and Measure (T.M.) circuit is also shown on the Low Temperature Schematic, Fig. 2. During this sequence the magnet is brought to the ISABELLE maxi-. mum design temperature of $3.8 \mathrm{~K}$ and is then energized at this temperature. The magnet is first "trained" to achieve the 
required field strength by successive quenches. During this training phase part of the stored magnetic energy is released to the supercritical helium refrigeration system. When the magnet has achieved design field strength, magnetic measurements are performed during steady state conditions. These measurements are made for two reasons: 1) to determine whether the magnet performance meets the required stringent tolerances; and 2) to precisely establish the location of the magnetic field relative to the warm vacuum tank so that the magnet can later be accurately positioned and surveyed into the ISABELLE ring.

A liquid helium precooler and subcooler are used to cool the supercritical helium gas which is circulated to the magnet via the T.M. circuit headers. The subcooler is pumped by an ejector which uses the high pressure return gas stream, expanding through a nozzle. 1 Two modes of operation are indicated on Fig. 2.: In Mode "A" (shown bold) the JT flow goes directly to the load at about $15 \mathrm{~atm}$; in Mode "B" a cold circulating pump is provided which sends supercritical helium in a closed loop through the subcooler bath, to the magnet and back. Mode "A" is simpler, but the flow rate is.Iimited to the refrigerator JT flow (approximately $55 \mathrm{~g} / \mathrm{s}$ ). The circulating pump for Mode "B" is designed to achieve the ISABELLE design flow rate of $170 \mathrm{~g} / \mathrm{s}$, at a pressure of 5.0 atm. ${ }^{2}$

During the "training" sequence of the magnet about 1 megajoule of energy is released when the magnet quenches. liter volume surge tank is provided to store the cold gas evolved when the magnet is quenched. An eutomatic system of valves and pressure sensors allows this gas to return to the system through $\mathrm{HX} \mathrm{C} 2 \mathrm{~B}$ making use of available sensible cooling capacity from the stored quench gas.

A small portion of the supercritical helium flow to the magnet is used to cool the current leads for the magnet; this gas flow is automatically controlled and returned warm to the compressor system. ${ }^{3}$

WARM-UP

During warm-up to room temperature the same temperature gradient limits as discussed previously for Cooldown I apply. For warm-up of the magnet, a third screw compressor is used to circulate helium gas through tempering heat-exchangers to the magnet and back. The heat exchangers are set up symetrically for Cooldown I and for Warm-up: Heat exchangers previously used for a Cooldown I cycle are switched for harm-up on the next cycle, and vice versa. Thus "cold" heat-exchangers from a previous cooldown are used for warm-up, providing tempered gas and 
avoiding excessive temperature gradients across a magnet during the warm-up cycle to room temperature.

The magnet heat shield is warmed by nitrogen gas which can be heated by an external electrical heater. The nitrogen gas is purged out of the shield circuit when the magnet warm-up is completed and replaced with pure helium.

\section{INSTRUMENTATION}

The complexity of MAGCOOL with its large number of process variables and control loops requires a sophisticated control system. Cryogenic systems are often complex because a large number of subsystems must operate in parallel to produce the desired result. The MAGCOOL system, in addition to subsystem control, must regulate, control, and monitor the. five magnet test stands through asynchronous cryogenic cycles. If a conventional analog control system were utilized, many operators would be required making the system very costly and dificult to operate.

For simpler operation and higher reliability a commercially available process control computer system was selected to perform the control tasks. The computer control system was specified, built and purchased to interface the MAGCOOL instrumentation. The input/output gear drives 178 valves, controls 38 direct digital control loops and reads over 100 assorted analog inputs. Conventional analog stations are not needed since all display and control is accomplished through a color graphics terminal. The comercial package includes all necessary system software; "CRISP" process control language for applications programing; cursor controlled CRT display building from the operators keyboard; scrolled trending; alarm and scale routines; battery backup of main memory; FORTRAN and BASIC high level programming languages.

The one area where comercial process control capability deviates from cryogenic needs is in temperature measurement and display. Since the normally supplied 12 bit analogue to digital conversion is not sufficient for high accuracy thermometry, this task was handled separately. A high resolution low drift microprocessor was designed to linearize and convert temperature inputs to engineering units. Temperature data is then transmitted to the process computer via a serial data link.

The process control computer has been tested for the past several months. During this period the system has been completely checked out and the beginnings of MAGCOOL applications software installed. Future operators of the system have 
been getting some initial training at the keyboard and have enthusiastically accepted this more modern form of control.

\section{ACKNOWLEDGEMENTS}

Thanks to the ISABELLE design group, especially J. J. Agostine and R. S. Meier for their assistance, and also to the ISA Cryogenic Instrumentation group.

- The magnet electrical cryogenic interconnections are provided by the ISA Magnet Division, under the direction of $D$. W. Gardner.

Many concepts and ideas have evolved from earlier forced flow tests performed by the ISA Cryogenic Group under H. Hildebrand's direction.

The MAGCOOL system is fabricated to BNL specifications ${ }^{4}$ by CVI, Inc., Columbus, Ohio. The ejector design and fabrication is by $T$. R. Strobridge, National Bureau of Standards, Boulder, Colorado.

The process control system is built to BNL specifications 5 by Anaconda Advanced Technology, Inc., Dublin, Ohio using a Digital Equipment Company LSI-11/23 computer.

\section{REFERENCES}

1. J.A. Rietdijk, The Expansion Ejector, A New Device for Liguefaction and Refrigeration at $4 \mathrm{R}$ and Lower, Proceedings of the International Institute of-Refrigeration, Commission I (Boulder, Colo. 1966).

2. D.P. Brown, et al., Design of $24.8 \mathrm{~kW}, 3.8 \mathrm{~K}$ Cryogenic System for ISABELLE, Paper to be presented at this Cryogenic Engineering Conference (San Diego, 1981).

3. D.P. Brown and W.J. Schneider, Magnet Leads for the First Cel1, Advances in Cryogenic Engineering, Volume 25, (Proceedings of the 1979 Cryogenic Engineering Conference).

4. J.A. Bamberger, Specification for ISABELLE Magnet Production Cooling Facility MAGCOOL, Brookhaven National Laboratory ISA Specification No. 35 (October 1979).

5. J.H. Sondericker, Specification for the ISABELLE magnet Production Cooling Facility Process Control Computer, Brookhaven National Laboratory ISA Specification No. 44 (January, 1980). 
D-51

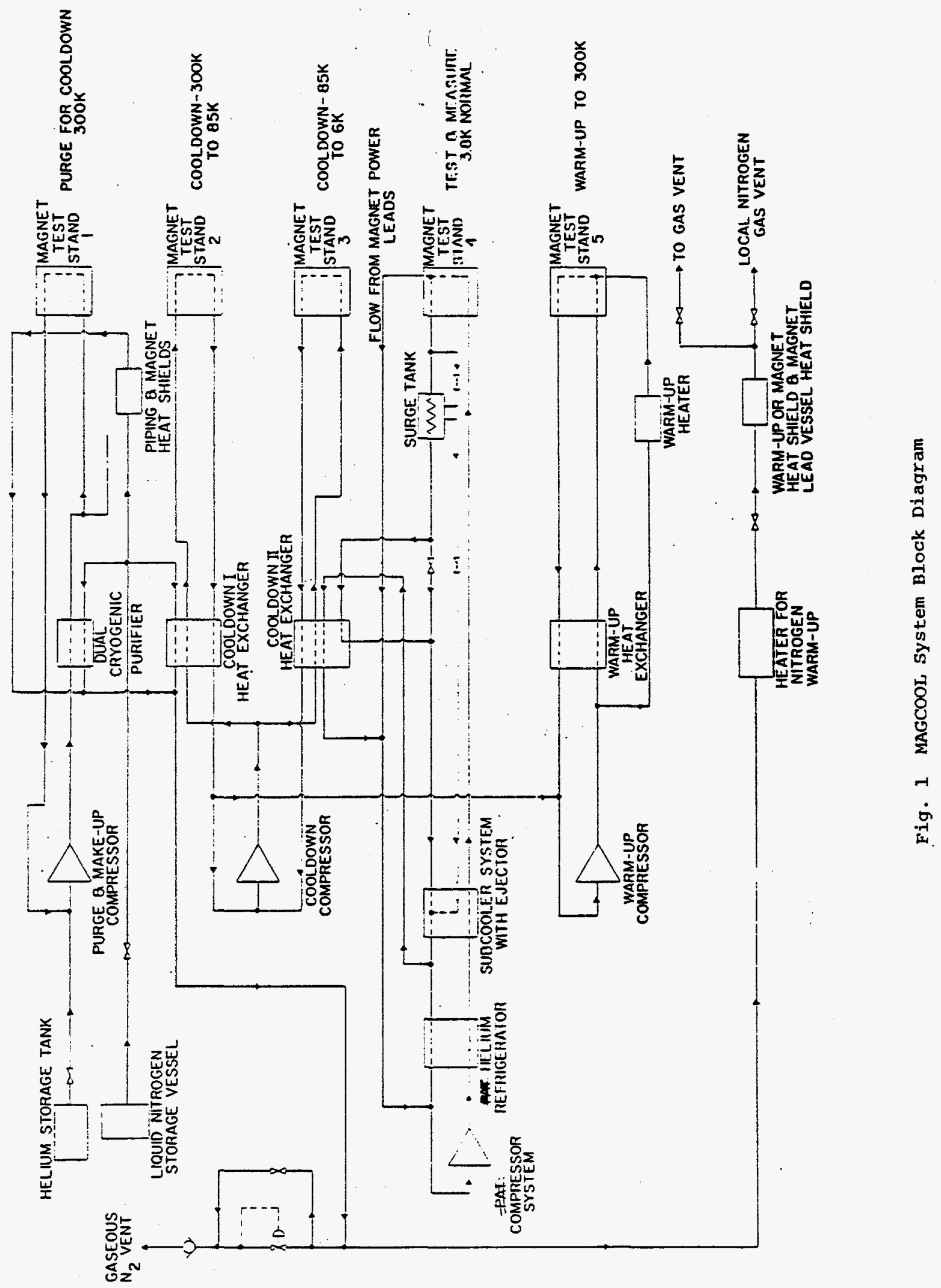


PAT

COMPRESSO
WARM RETURN

LEAD GAS RETUR

PAF COLO

RE TURN SUPPLY
SUPO

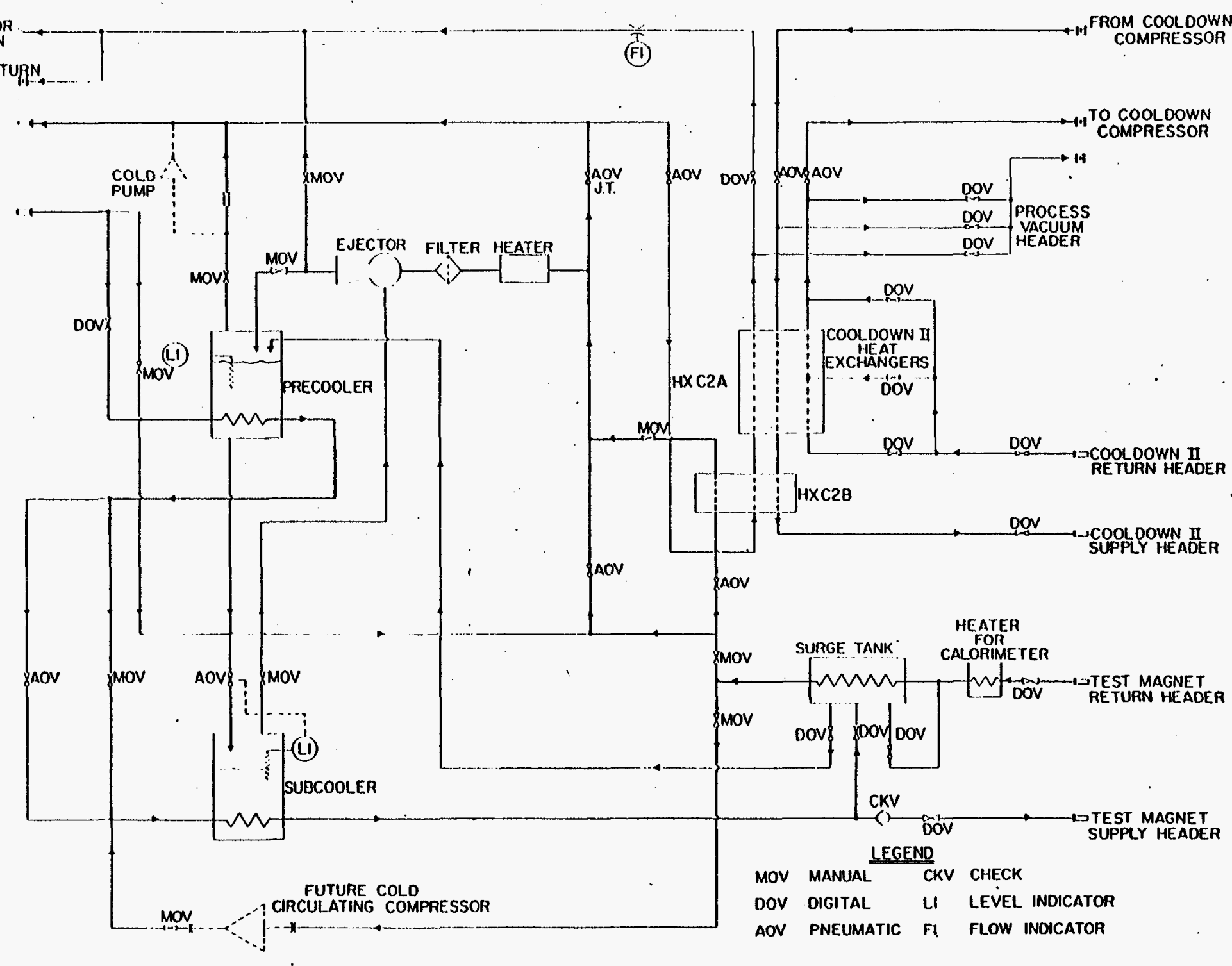

Fig. 2 MAGCOOL Low Temperature Flow.Schematic 
AUTOMATION OF A CRYOGENIC FACIIITY BY

COMERCIAL PROCESS CONTROL COMPUTER*

John H. Sondericker, D. Campbel1, and D. Zantopp

B rookhaven National Laboratory

Upton, New York

\section{INTRODDCTION}

To insure that Brookhaven's superconducting accelerator magnets are reliable and their field quality meets accelerator requirements, each magnet is pre-tested at operating conditions after construction. MAGCOOL, the production magnet test facility, 1 was designed to perform these tests, having the capacity to test ten magnets per five day week. This paper describes the control aspects of MAGCOOL and the advantages afforded the designers by the implementation of a commercial process control computer system.

\section{MAGCOOL DESCRIPTION}

The magnet test facility is more complex than most cryogenic systems. It has all the conventional components (compressors, purifiers, cold boxes) plus complicated supply and return headers that permit as many as five superconducting magnets to be cycled through cooldown, test and warmup steps asynchronously.

Figure I shows a simplified process flow schematic of the system. A normal magnet testing cycle consists of five functional steps. They are:

Pump and Purge - replaces air in magnet with purified helium Cooldown 1 - cools magnet from room temperature to $90 \mathrm{~K}$ Cooldown 2 - cools magnet from $90 \mathrm{~K}$ to $10 \mathrm{~K}$

\footnotetext{
*Work performed under the auspices of the U.S. Department of Energy.
} 


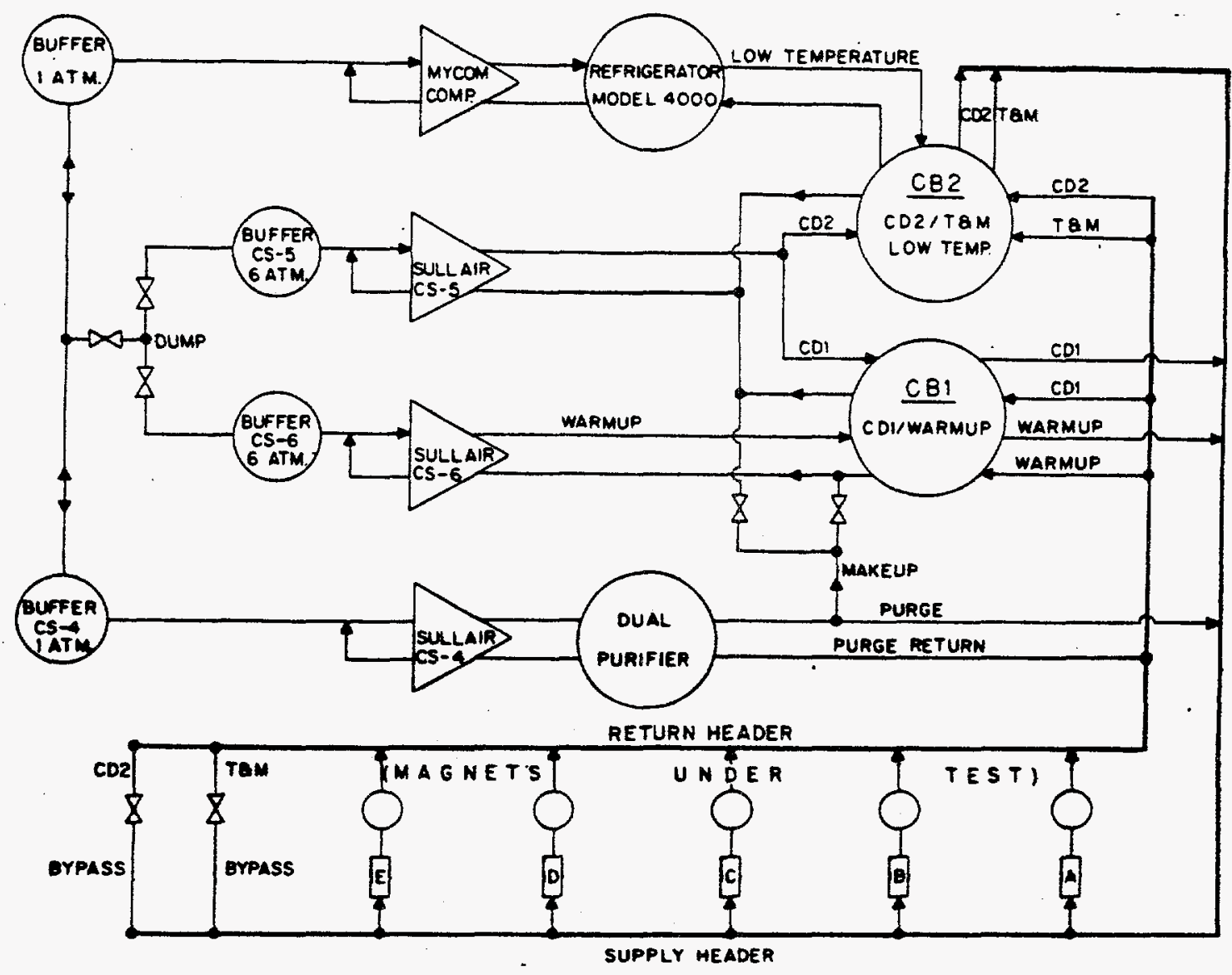

Fig. 1. Simplified MAGCOOL Process Flow.

Test and Measure - magnet cooled to $3.9 \mathrm{~K}$, quenched and field measured.

Warmup - Magnet warmed to room temperature

Cold Box 1 ( $C B 1$ ) contains heat exchangers to perform cooldown 1 and warmup tasks. Cold Box 2 (CB2) contains the precooler/subcooler and heat exchangers for cooldown 2 and the subcooled helium test and measure functions. Supply and return headers consist of a group of parallel transfer lines plus over one hundred stop and control valves that permit a magnet in any test stand to be connected to selected hardware so that the load can be cycled through the five functional test steps. Process I/O consists of a total of over 350 points which include 30 analog outputs used for direct digital closed loop control. Liquid nitrogen shielding is used to reduce heat leak to the portion of the system that operates at or below liquid helium temperature. 


\section{CONTROL SYSTEM DESCRIPTION}

\section{Instrumentation and Control Philosophy}

Unlike most cryogenic systems which go through a series of steps to get to operating conditions, many of MAGCOOL's functions are continually dynamic and parallel in nature. During the early design stages of the system it was clear that control would be difficult and would require at least two, probably three operators per shift if the usual analog panel approach was used. Operator error was a worry because with five magnets cycling through five functions in five test stands many tens of valves would have to be manipulated per shift. At best, errors would cause only lost time but some process variables have strict operational limits that set magnet maximum cooldown and warmup rates to keep internal stress within safe limits. Clearly, a computer based control system that could protect the magnets from operator error and over stress would be beneficial. The decision was made to minimize operator intervention, reduce operator error and manpower requirements by totally automating the system. The philosophy was extended by designing MAGCOOL instrumentation to interface specifically to computer $I / O$ and to rely on the digital system to perform all control, alarming and logic sequencing without aid of analog meters or controls except where they were embedded as part of commercial equipment.

\section{The Buy or Build Decision}

There is always a temptation when embarking on a project such as MAGCOOL to buy a digital processor, color CRT and I/O cards from suppliers and construct one's own system. In theory, the designer has the option to add "state-of-the-art" features such as fast data highways, distributed microprocessors and extended graphic packages to tailor the electronics to the exact needs of the process system. An alternative approach is to purchase a comercially supplied process control computer system and modify it, where necessary, for cryogenic control. The advantages realized by this approach are:

1. A comercial process system should be a bug-free fieldproven product; a mature system, the design a result of many iterations.

2. The process engineer can concentrate on cryogenic application software and not be burdened with hardware and software systems development. 
3. Implementing a commercial system will, in the end, be less expensive than constructing a custom system inhouse.

4. Probably most important, software and hardware system documentation and support is the responsibility of the supplier, not the user.

Approximately fifteen manufacturer's quotes were evaluated and surprisingly almost all could furnish systems useable for cryogenic control tasks. The only cryogenic process variable that presented problems was high accuracy temperature measurement, which was solved by interfacing our own microprocessor to the system via a $20 \mathrm{ma}$ serial communication loop. Comercial system architecture ranged from the very simple to distributed processors using peer to peer local area network communications. Anaconda Advanced Technology's (Anatec) CRISP system was selected.

\section{System Description}

Hardware consists of a 256 kilobyte PDP 11/34 floating point processor, LA 120 printer, dual hard discs, appropriate $I / 0$ hardware and a high resolution 80 character by 48 line color graphics terminal. Two cabinets of $I / 0$ gear are electrically separated from the processor by use of opto isolated bus drivers. D.E.C.'s RSX-11M monitor was supplied which is a powerful multiprograming operating system ideally suited for real time control applications.

For application programing Anatec's CRISP high level process control language was provided. The process engineer uses CRISP to declare I/O points, perform logic, arithmetic, timing and counting operations and program closed loop DDC control strategies. All application software is resident in battery backed memory to protect from power or disc failure. The operator interface consists of the color graphics terminal upon which piping schematics, process variables, loop controllers, system trends and alarms are dynamically displayed in a paged format (Fig. 2). All CRT pages are easily built from the operator's keyboard by use of a menu of standard process or user configured symbols. Screen dynamic variables are linked to the CRISP data base to provide automatic refresh every $600 \mathrm{~ms}$. Existing displays can be modified or additional CRT pages can be constructed on line. The number of CRT pages is only limited by available disc space. 


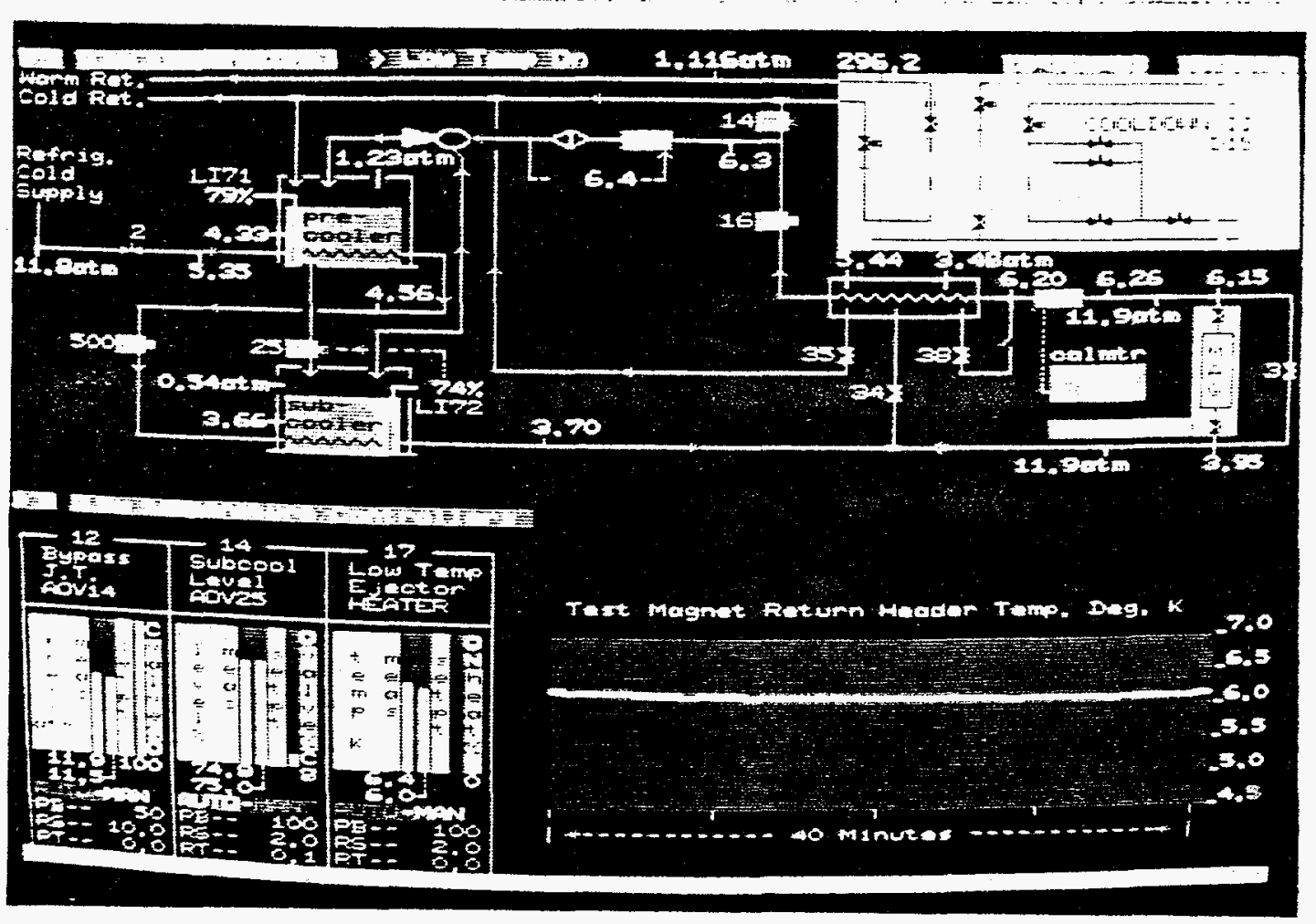

Fig. 2. Typical CRT Display.

\section{Control System Organization}

CRISP furnishes all the necessary tools but it is up to the designer to mold these features into a control system that the operator will accept and feel comfortable with. The operator must not become frustrated or intimidated while trying to interact with the process through the keyboard.

Control system design was divided into operator systems and application software. Operator systems included display design, alarm routines and deciding what convenience should be furnished to the operator so that he has flexibility while keeping the system safe from error. Application software, which was divided between utility and functional CRISP subroutines, proved to be the easier of the two tasks. Utility programs sequence and control compressors and purifiers, and perform gas handing duties which the operator has to initiate to get started but thereafter rarely looks at. Functional subroutines program the magnets through their five test stages.

To minimize intervention, the operator only has to start and stop the various functional subroutines to step the magnets 


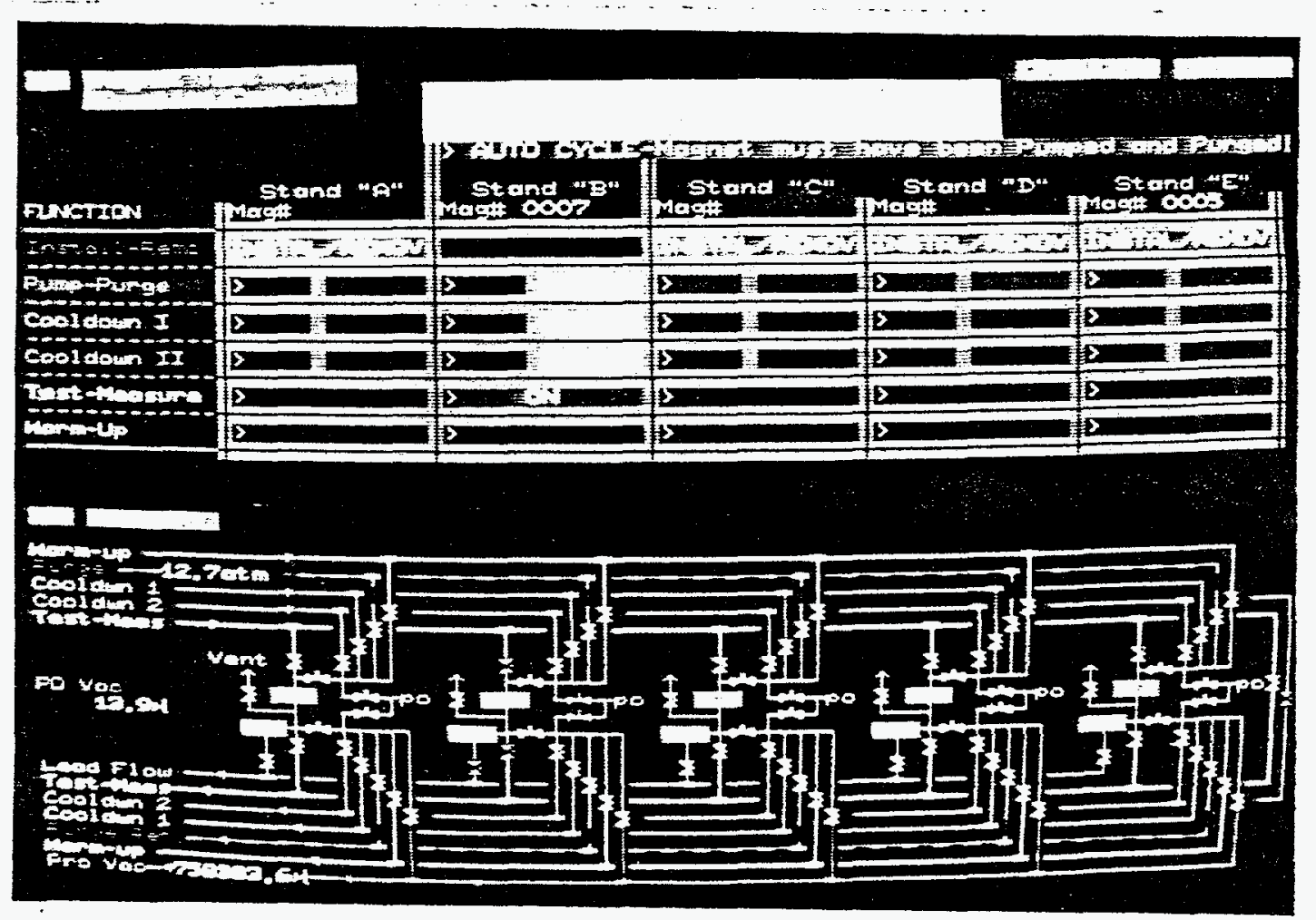

Fig. 3. Test Stand Function Control Page.

through their test cycles. Figure 3 shows the magnet test stand function control CRI page. Software interlocks prevent out of sequence or other test stand tasks from being activated. Once the subroutine is installed, all valves and controllers are automatically initiated and sequence through to the completion of the functional step. When warmup is done the subroutine turns itself off and automatically puts the magnet into a safe depressurized install-remove state.

If problems with hardware or software arise, the operator has the option to abort automatic and go to semiautomatic or full manual control. However, the system is so logically complex that this feature is rarely used. Time can be better spent by going off line, debugging in the manual mode, repairing the problem and coming back on line in automatic. Comments were heavily used throughout source code programming to aid in understanding the logic at some future time. CRISP logic and graphic displays took one man-year to complete. Of the total, four man-months were de- 
voted to applications programming and the remainder spent on CRT display and operator system interface design.

\section{OPERATING HISTORY}

The control system was programined and connected to MAGCOOL hardware during mechanical installation. Software was debugged by turning off computer I/O scan and setting dumy process variables from the operator's keyboard.

MAGCOOL was brought on line in February, 1983. Compressor controls, gas management and purifier reactivation utility programs worked as expected with only minor PID control loop parameters needing adjustment. A day later a test magnet was pumped and purged and started into cooldown 1. Even though cooldown 1 is logically very complex and difficult to control, CRISP performed beyond expectations. Basically, all software and hardware of the remaining functions executed equally well with only a few minor changes that were accomplished on line. The system not only worked but exceeded expectations on the first try.

\section{SUMMARY}

To date over ten magnet test cycles have been programed through MAGCOOL. The system has seen a few power and hardware failures plus some leaking valves but is flexible enough in design to absorb problems and continue to perform. Operators now prefer the CRT and keyboard to the analog panel and want to know more of the details of programing the system.

While many control engineers will argue that abandoning ana$\log$ black boxes in favor of digital control is risky, MAGCOOL's operating record has shown that operator error has been sharply reduced, one man operates the entire system and a vast improvement in magnet test cycle time has been realized as compared to our older analog system which performs the same tasks on a single magnet.

Using a comercially available process control computer system for cryogenic control can save time, manpower and money; all steps in the right direction. 


\section{REFERENCE}

1. J. A. Bamberger, et al., "MAGCOOL" - The production cooling facility for Isabelle magnets, in: "Advances in Cryogenic Engineering, Vol. 27," Plenum Press, New York (1982), p. 533. 
Presented at the 1981 Cryogenic Engineering Conference, San Diego, California, August 10-14, 1981.

\section{ANALYSIS OF COOLDOWN PERFORMANCE FOR ISABELLE HELIOM REFRIGERATOR*}

K. C. Wu and D. P. Brown

Accelerator Department Brookhaven National Laboratory

Upton, New York 11973

R. W. More

Roch Process Systems

Westborough, Mas sachuse tts 01581

\section{INTRODUCTION}

The ISABELLE refrigeratorl was designed to provide cooling for 1000 superconducting magnets. It has the dual duty of providing cooling at steady-state operating conditions and to cooldown massive magnets from ambient temperature. During the cooldown, tremendous quantities of heat $\left(s_{4.8} \times 10^{11} \mathrm{~J}\right)$ must be removed from the magnets $\left(5.9 \times 10^{9} \mathrm{gm}\right.$ of iron) and 75,000 liters of cold helium must be supplied to fill the system. In the planning for ISABELLE, this procedure has been estimated to require approxinately two weeks. Because of this long cooldown time and the fact that during cooldown the refrigerator will be operated quite differently from its steady-state design configuration, it is important to maximize the cooling capacity and identify the cooldown procedure.

\section{RELATIONSHIP BETWEEN A REFRIGERATOR AND ITS LOAD}

A cooldown analysis involves tching perfomance be tween the refrigerator and the load. The cooling process can be

*Work performed under the auspices of the U.S. Department of Energy. 
characterized by three parameters: 1) the rate at which heat is removed from the load or apparent refrigeration, 2) the rate at which helium is deposited in the load or apparent liquefaction rate and 3) the temperature at which the process is carried out.

The capacity of the refrigerator may be 1 inited by any cowponent in the refrigerator, but the most common limits are compressor capacity, expander throughput and heat exchanger surface. Within operating ranges, the refrigeration effect and apparent liquefaction rate can be traded for each other. A performance curve for the refrigerator could be drawn on a liquefaction versus refrigeration figure as given in Fig. 1.

The load behaves oppositely to the refrigerator during cooldown with apparent liquefaction rate directly proportional to the apparent refrigeration as shown in Fig. 1. This can be understood based on the simple physical ground that the more cooling a refrigerator provides, the faster the load will be cooled down and the more rapidly helium will be accumulated in the ioad. The intersecting point for the two curves determines the operating point at that particular temperature.

\section{CYCLE DESCRIPTION AND PROCEDURE FOR COOLDOWN}

The ISABELIE refrigerator has five turboexpanders in its cycle. Four of the five have a redundant mate provided. A flow diagram is shown in Fig. 2. During steady-state operation there is no excess compressor flow to drive the redundant expanders. In cooldown operation, flow can be allocated for the redundant expanders by rearranging the flow inside the refrigerator or by

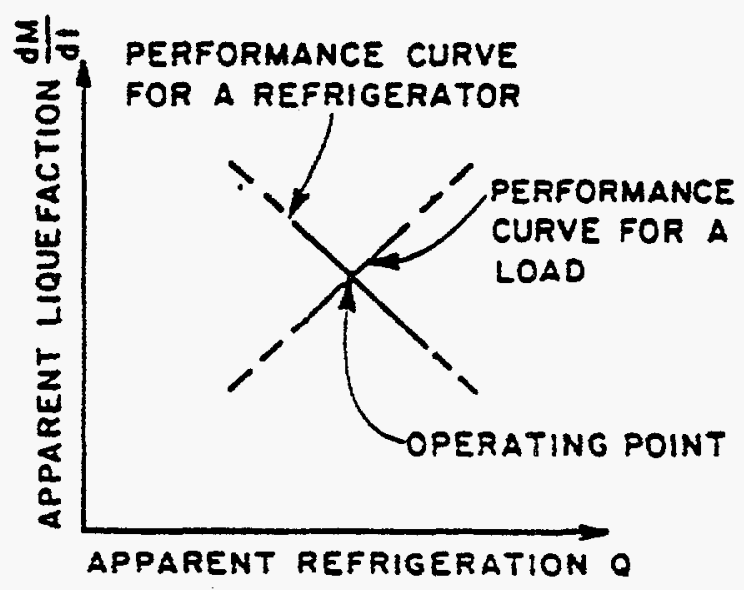

Fig. 1. Relationship between a refrigerator and its load. 
us ing helium returned from the gaet. The latter is permitted because the ISABELLE magnet system is designed for 20 atm pressure and a small pressure drop is anticipated through the load.

For initial stage of cooldown, the magnet is warm and the return helium cannot be used for cooling generation. The refrigerator behaves as a liquefier. During this stage, two sets of expanders 1 and 2 in parallel and one set of expanders 3 and 4 will be operated. High pressure ( $15 \mathrm{~atm}$ ) helium from the outlet of heat exchanger 8 will be used to cool the headers and magnets. The return gas will be introduced into the cryogenic purifier until the temperature reaches approximately $200^{\circ} \mathrm{R}$.

As the return temperature becomes lower than $200^{\circ} \mathrm{K}$, helium will be introduced to the proper redundant turbine. Five locations with their operating temperatures are shown in Fig. 2 . The switch of return helium from one turbine to the other is similar to the switch of return helium from one heat exchanger to the other in the cooldown operation of a conventional helium refrigerator. Proper throttling of flow through each turbine is required to intain reasonable temperatures throughout the refrigerator. Apparent liquefaction rate and refrigeration supplied mat also be balanced by holding the magnet system pressure constant.

When liquid helium temperature is reached at the outlet of turbine 5, the refrigerator will be switched into its steadystate configuration. Circulating compressor is then started and liquid level in the pots of the subcooler must be maintained. When the average temperature in the magnet is around $5.5^{\circ} \mathrm{R}$, the pressure in the magnet will be allowed to fall along a constant density line with decreasing temperature as shown in Fig. 3 until the 5 atm operating condition is reached. Finally the two vacum compressors are turned on and steady-state performance is reached.

\section{MODELING THE REFR IGERATOR}

We assume quasi-steady-state operation, so the problem for modeling cooldown is the same as other of f-design computations for the refrigerator. The method employed in the present anal$y s$ is is based on solving terminal temperature relationships among constituent heat exchangers and turbines according to theil conventional definitions.

The terminal temperatures for heat exchangers are related by the effectiveness and energy equations. 2 The inlet and outlet conditions for a turbine are related by the adiabatic efficiency. With given flow distribution, the AU (heat transfer 


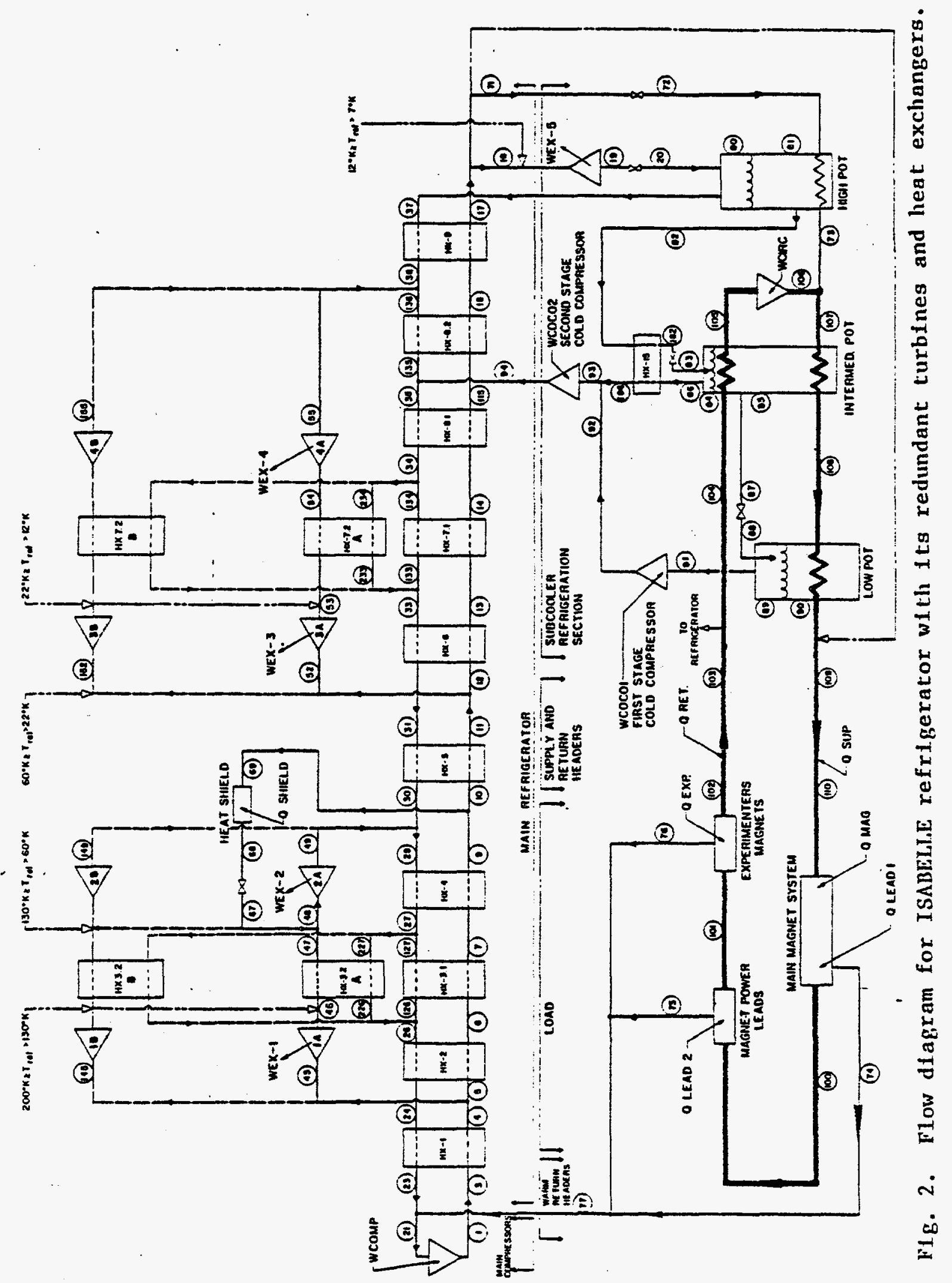


area times overall heat transfer coefficient) or NTU (no. of transfer units) for the heat exchangers and $n$ for turbines, solutions can be obtained by inverting the temperature relationship matrix with iteration against specific heat.

Typical perforance for a turbine, with efficiency versus flow rate at selected inlet temperature is given in Fig. 4. For simulating heat exchanger performance, we as sume design AU for heat exchangers with flow rate greater than design value and design NTU for those flow rates less than design value. This results in a conservative prediction for performance.

\section{CONCEPTS OF MAXIMIZING COOLDOWN CAPACITY}

For proper operation of a refrigerator, both refrigeration, I iquefaction and load temperature must be considered at the same time. For the initial stage of cooldown, one could produce more refrigeration (or work extraction) by expanding helium across a fixed pressure drop at higher.temperature than at lower temperature. For example $1 \mathrm{~g} / \mathrm{s}$ of helium produces $345 \mathrm{~W}$ in warm expander 1 and 2 , but only $55 \mathrm{~W}$ will be produced in expander 3 and 4. Six times more cooling could be obtained by al locating helium from EX3 and EX4 into EXI and EX2. Therefore, in the initial stage of cooldown, both sets of EXI and EX2 should be operated at their full flow capacity to obtain the maximum refrigeration for that temperature.

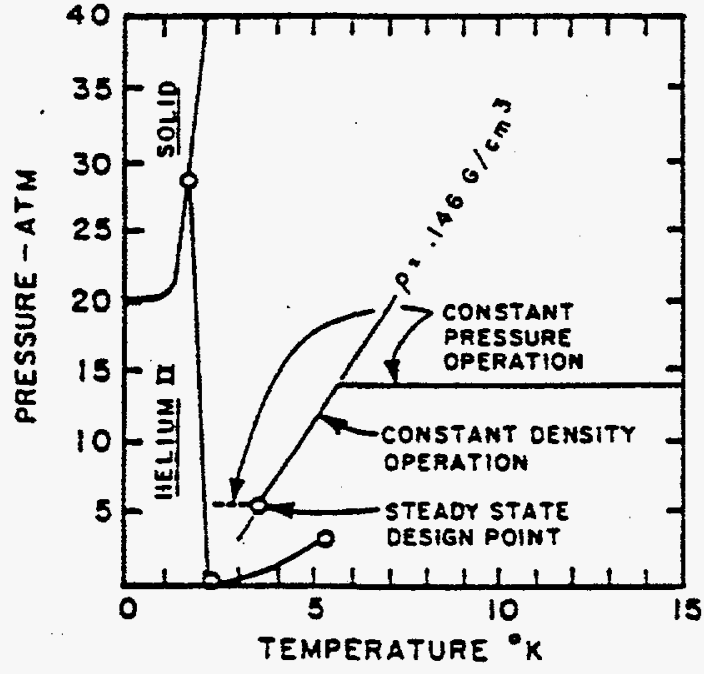

Fig. 3. Pressure vs. temperature for ISABELIE load

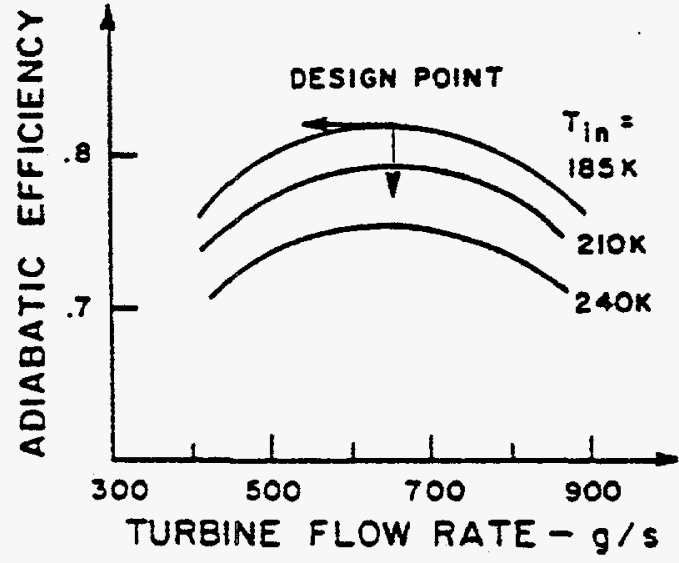

Fig. 4. Efficiency for turbine 1 
As temperature decreases, more helium will be allocated into EX3 and EX4 accompanied with $a$ reduction in EX1 and EX2. At this stage, the refrigeration could only be enhanced by us ing redundant EX3, EX4 and EX5 in series with the magnets.

\section{RESULTS AND DISCUSSION}

Cooldown calculation has been made for load return temperatures at $300^{\circ} \mathrm{K}, 175^{\circ} \mathrm{K}, 85^{\circ} \mathrm{K}, 40^{\circ} \mathrm{K}, 25^{\circ} \mathrm{K}, 20^{\circ} \mathrm{K}, 15^{\circ} \mathrm{K}, 12^{\circ} \mathrm{K}$ and $10^{\circ} \mathrm{K}$. Apparent liquefaction rates between $80 \mathrm{~g} / \mathrm{s}$ and $400 \mathrm{~g} / \mathrm{s}$ are examined. At high temperature $\left(>85^{\circ} \mathrm{K}\right)$ a high ratio of refrigeration over liquefaction is anticipated due to the high specific heat of iron and the low density of helium in this region. As temperature decreases the ratio of refrigeration to liquefaction reduces correspondingly. For load temperature below $40^{\circ} \mathrm{R}$, the apparent liquefaction rate is estimated to be between $150 \mathrm{~g} / \mathrm{s}$ and $200 \mathrm{~g} / \mathrm{s}$ for the present analysis. A typical T-S (temperature-entropy) diagram for cooldown operation is given in Fig. 5. The apparent 1 iquefaction rate versus refrigeration rate for $\mathrm{T}_{\text {ret }}<40^{\circ} \mathrm{K}$ is plotted on Fig. 6 . The capacity curve for the refrigerator shifts to the left and tends to the steady-state capacity as temperature decreases.

In conclusion, the ISABELIE refrigerator with its redundant expanders properly used achieves capacity well beyond its steady-state capacity. During the initial stage of cooldown, by utilizing the two warmest redundant expanders the refrigerator can generate 2.3 times steady-state refrigeration above $50^{\circ} \mathrm{K}$, which is comparable to a typical liquid nitrogen precooled refrigerator. As the temperature decreases, a significant portion of the refrigeration is generated through use of redundant expanders 3 and 4 and expander 5 in series with the magnets. After the refrigerator goes into its steady-state operation, the capacity of the refrigerator tends to its steady-state capacity. The cooldown rate at this stage relies on the design safety margin. For the ISABEILE refrigerator, the safety margin is $50 \%$.

\section{REFERENCES}

1. D.P. Brown, A.P. Schlafke, R.C. Wu and R.W. Moore "Cycle Design for the ISABELLE Belium Refrigerator," Paper presented at 1981 Crgogenic Engineering Conference, San Diego, CA, Aug. 10-14, 1981 .

2. W.M. Rays and A.L. London, "Compact Heat Exchangers," The National Press, Palo Alto, Calif., 1955. 


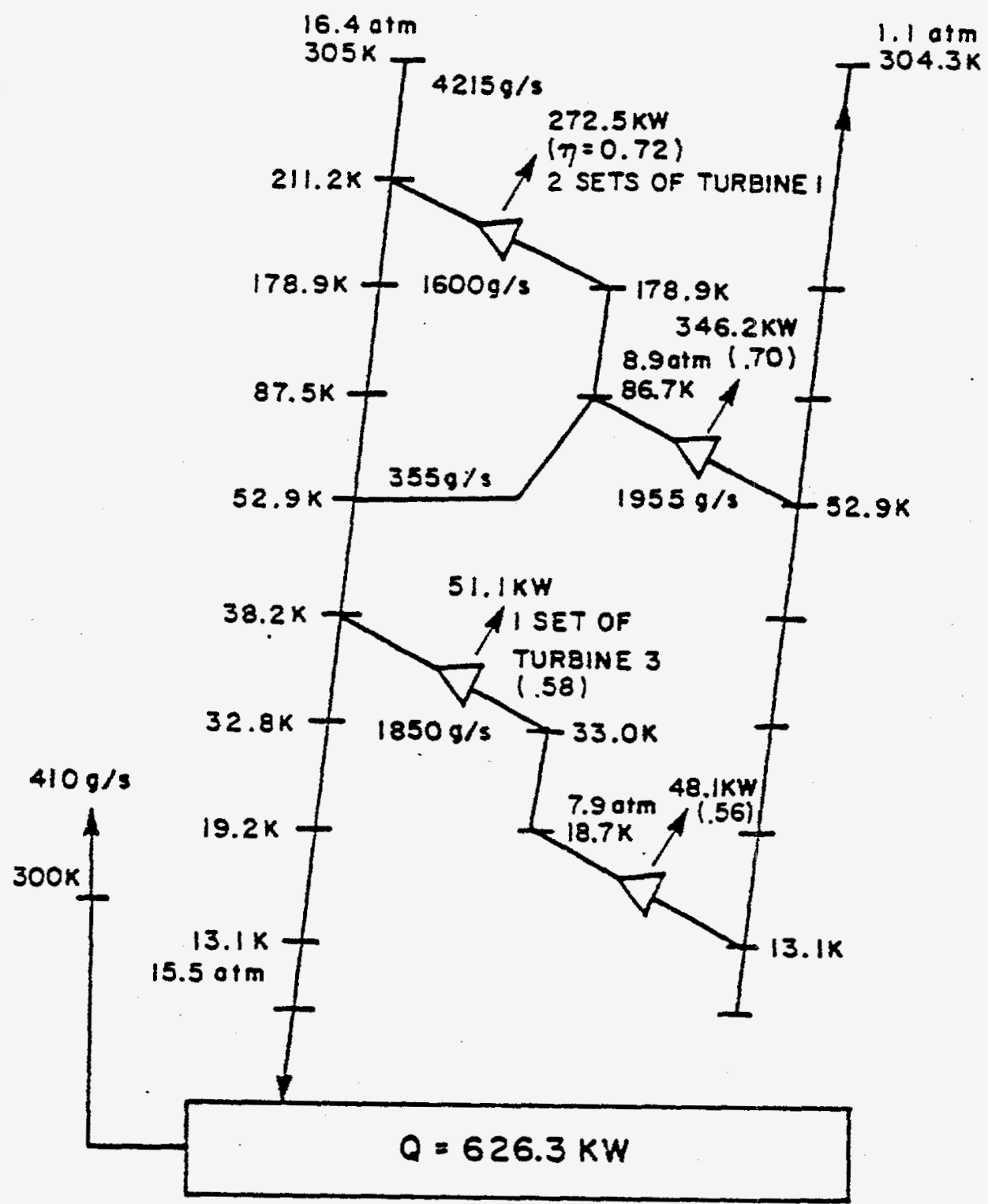

Fig. 5. I-S diagram for initial stage of cooldown 


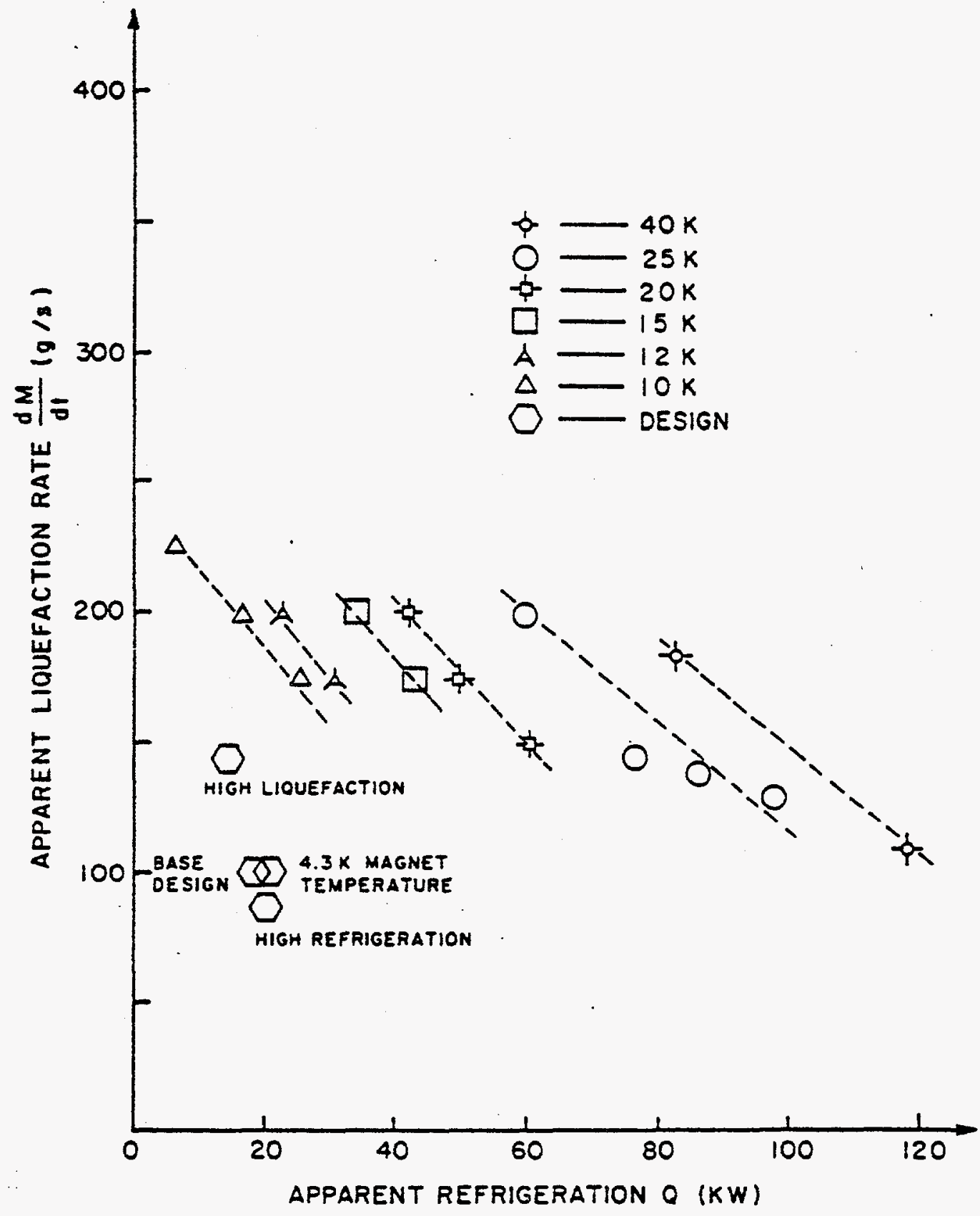

Fig. 6. Refrigerator characteristic at various return tempera ture 


\begin{tabular}{c} 
LONG STRING \\
Materia1 \\
K\$ \\
\hline
\end{tabular}

2- He/Air HX

3- Piping \& Valves

4- Installation

$5-$ lst charge $\left(500,000 \mathrm{ft}^{3}\right)$

.6 Cooldown $\mathrm{HX}$

.7 Calorimeter/Warm-up Heater

.8 Process Vac. Pump

.9 LHe Storage Dewar (5,000 1iter)

$.10 \mathrm{LN}_{2}$ Storage

3- $\mathrm{LN}_{2}$ Pump

4- Valves \& Piping

1.4.2.2.4.1 He Transfer Lines

1 Supply \& Return \& \$100 m

2 Warmup/Cooldown

3 Utility \& Insertion

4 Installation $10 \mathrm{~m}-\mathrm{hr} / \mathrm{m}$

1.4.2.2.5.1 Warm Return System

1 Lead FLow Controls

2 Relief Valves

3 Main Header 4"

4 Connections

5 Instrumentation

6 Expansion Joints

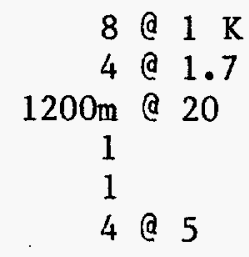

.2 Supply
BNL

BNL

BNL

BNL

BNL

10

\author{
SHORT STRING \\ Material \\ $\mathrm{KS}$
}

BNL

BNL

BNL

BNL

BNL

BNL

BNL

100

2

10

20

15

Not Req'd.

4

4

$\mathrm{T} O \mathrm{TAL}$

$\$ 145 \mathrm{~K}$

$\$ 123 \mathrm{~K}$ 
D-70

.2 Distributed Remote Control

1. Processor Upgrade

50

.3 Ring Resident Instrumentation

1. Spec. Temp. Interface

10

2. Lead Flow Controllers

12

3. Wiring, Checkout

10

4. Temp. Sensor Calib.

9

5. Misc. Interface Construction

20

TO TA L 
Cryogenics

Operating Crews

( $1 \mathrm{MT} \& 1 \mathrm{ET}$ ) $\mathrm{T} /$ shift $\times 2$ shifts/day

Helium Gas $\left(50,000 \mathrm{ft}^{3} \times 10 \mathrm{mo} . \mathrm{x} .06\right)$

( 5 full string quenches $\times 500,000 \mathrm{ft}^{3} \times 0.6$ )

\begin{tabular}{|c|c|c|}
\hline $\begin{array}{l}\text { KS } \\
\text { Mat'1 } \\
\end{array}$ & & \\
\hline & $\underline{P}$ & $\mathrm{~T}$ \\
\hline 60 & 1.0 & 6.0 \\
\hline $\begin{array}{r}30 \\
150\end{array}$ & & \\
\hline 50 & & \\
\hline $\begin{array}{l}270 \\
125\end{array}$ & & \\
\hline 30 & & 3.0 \\
\hline 10 & 1.0 & \\
\hline$\overline{725}$ & 2.0 & 9.0 \\
\hline 35 & 1.0 & 2.0 \\
\hline 6 & 0.6 & 0.6 \\
\hline 5 & 2.0 & \\
\hline$\overline{771}$ & 5.6 & 11.6 \\
\hline
\end{tabular}

Power Supplies and QPM

Data Collection

Test Coordination

Total

( 365 days $x .45$ C 68.1 \$/MWh)

5 cooldowns @ $\$ 25 \mathrm{~K}$

125

Preventive Maintenance

Data Analysis, etc.

Subtotal 
D-72

K\$

Mat'1

\section{Cryogenics}

Operating Crews

( 1 MT \& 1 ET) T/shift $x 2$ shifts/day

Helium Gas $\left(50,000 \mathrm{ft}^{3} \times 10 \mathrm{mo} \times \mathrm{x} .06\right)$

( 5 full string quenches $x 135,000 \mathrm{ft}^{2} \times .06$ )

$\mathrm{LN}_{2}$ (100 1iter/hr.x300 days @ .07 \$/1iter)

Elec. Power ( 365 days $x .45 @ 68.1 \$ / M W h$ ) 5 cooldowns @ $\$ 12 \mathrm{~K}$

Preventive Maintenance

Data Analysis, etc.

Subtotal

Power Supplies and QPM

Data Collection

Test Coordination

Total

\begin{tabular}{|c|c|c|c|}
\hline Cryogenics & & $\underline{\mathbf{p}}$ & $\mathrm{T}$ \\
\hline $\begin{array}{l}\text { Operating Crews } \\
\quad(1 \mathrm{MT} \& 1 \mathrm{ET}) \mathrm{T} / \text { shift } \times 2 \text { shifts/day }\end{array}$ & 60 & 1.0 & 6.0 \\
\hline $\begin{array}{l}\text { Helium Gas }\left(50,000 \mathrm{ft}^{3} \times 10 \mathrm{mo} \times .06\right) \\
\quad\left(5 \text { full string quenches } \times 135,000 \mathrm{ft}^{2} \times .06\right)\end{array}$ & $\begin{array}{l}30 \\
40\end{array}$ & & \\
\hline $\mathrm{LN}_{2}$ (100 1iter $/ \mathrm{hr} \cdot \times 300$ days @ $.07 \$ / 1$ iter $)$ & 50 & & \\
\hline $\begin{array}{c}\text { Elec. Power ( } 365 \text { days } x .45 @ 68.1 \$ / M W h \text { ) } \\
5 \text { cooldowns @ } \$ 12 \mathrm{~K}\end{array}$ & $\begin{array}{r}270 \\
60\end{array}$ & & \\
\hline Preventive Maintenance & 60 & & 3.0 \\
\hline Data Analysis, etc. & 10 & 1.0 & \\
\hline Subtotal & $\overline{550}$ & 2.0 & 9.0 \\
\hline Power Supplies and QPM & 35 & 1.0 & 2.0 \\
\hline Data Collection & 6 & 0.6 & 0.6 \\
\hline Test Coordination & 5 & 2.0 & \\
\hline Total & $\overline{596}$ & 5.6 & $\overline{11.6}$ \\
\hline
\end{tabular}

M-Yr.

Labor

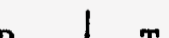

.0

3.0 


\begin{tabular}{|c|c|c|}
\hline $\begin{array}{l}\mathrm{K} \$ \\
\mathrm{Mat} ' 1 \\
\end{array}$ & & \\
\hline & $\mathbf{P}$ & $\mathrm{T}$ \\
\hline 60 & 1.0 & 6.0 \\
\hline $\begin{array}{l}30 \\
20\end{array}$ & & \\
\hline 50 & & \\
\hline $\begin{array}{r}270 \\
45\end{array}$ & & \\
\hline 60 & & 3.0 \\
\hline 10 & 1.0 & \\
\hline$\overline{515}$ & 2.0 & 9.0 \\
\hline 35 & 1.0 & 2.0 \\
\hline 6 & 0.6 & 0.6 \\
\hline 5 & 2.0 & \\
\hline$\overline{561}$ & 5.6 & 11.6 \\
\hline
\end{tabular}

Cryogenics

Operating Crews

(1 MT \& $1 \mathrm{ET}) \mathrm{T} / \mathrm{shift} \times 2$ shifts/day

Helium Gas $\left(50,000 \mathrm{ft}^{3} \times 10 \mathrm{mo} \cdot \mathrm{x} .06\right)$

( 5 full string quenches $x 67,500 \mathrm{ft}^{2} \times .06$ )

$\mathrm{LN}_{2}$ (100 1iter/hr.x300 days e .07 \$/1iter)

Elec. Power (365 days $x .45$. $68.1 \$ /$ MWh) 5 cooldowns \& $\$ 9 \mathrm{~K}$

Preventive Maintenance

Data Analysis, etc.

Subtota1

Power Supplies and QPM

Data Collection

Test Coordination

Total

HAVEN

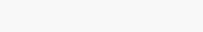


KS

$\underline{\text { Mat'1 }}$

Cryogenics

Operating Crews

Helium Gas $\left(25,000 \mathrm{ft}^{3} \times 12\right.$ mo. $\left.\times .06\right)$

$\mathrm{LN}_{2}$ (160 1iter/hr.x365 days @ .07 \$/1iter)

Elec. Power (365 daysx.56 MW @ 68.1 \$/MWh)

Preventive Maintenance

Data Analysis, etc.

Subtotal

Power Supplies and QPM

Data Collection

Test Coordination

Total
M-Yr.

Labor

20

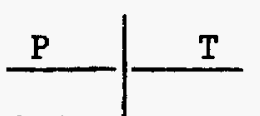

18

98

335

10

\begin{tabular}{cc|c}
5 & 0.4 & \\
\hline 486 & 0.5 & 2.0 \\
25 & 1.0 & 2.0 \\
4 & 0.4 & 0.4 \\
5 & 2.0 & \\
\hline 520 & 3.9 & 4.4
\end{tabular}




\section{OPERATING COSTS PER YEAR}

$\mathrm{K} \$$

Mat'1

Cryogenics

Operating Crews

(2 MT \& 2 ET)

Helium Gas $\left(25,000 \mathrm{ft}^{3} \times 12\right.$ mo. $\left.\times .06\right)$

$\mathrm{LN}_{2}$ (105 liter/hr.x365 days @ .07 \$/1iter)

Elec. Power (365 daysx.56 MW @ $68.1 \$ / M W h$ )

Preventive Maintenance

Data Analysis, etc.

Subtota1

Power Supplies and QPM

Data Collection

Test Coordination

Total

\begin{tabular}{rr|r} 
& $\mathrm{P}$ & $\mathrm{T}$ \\
\hline 20 & 0.1 & 4.0 \\
18 & & \\
64 & & \\
335 & & \\
10 & & \\
5 & 0.4 & \\
\hline 452 & 0.5 & 4.0 \\
25 & 1.0 & 2.0 \\
4 & .4 & 0.4 \\
5 & 2.0 & \\
\hline 486 & 3.9 & 6.4
\end{tabular}

Labor 
D-76

Mat'1

Cryogenics

Operating Crews

(2 MT \& $2 \mathrm{ET}$ )

Helium Gas $\left(25,000 \mathrm{ft}^{3} \times 12 \mathrm{mo.} \times .06\right)$

$\mathrm{LN}_{2}$ (150 1iter/hr.x365 days @ .07 \$/1iter)

E1ec. Power (365 daysx. 56 MW a 68.1 \$/MWh)

Preventive Maintenance

Data Analysis, etc.

Subtotal

Power Supplies and QPM

Data Collection

Test Coordination

Total
$\mathrm{K} \$$

M-Yr.

Labor

20

18

92

335

10

\begin{tabular}{rr|r}
5 & 0.4 & \\
\hline 480 & 0.5 & 4.0 \\
25 & 1.0 & 2.0 \\
4 & .4 & 0.4 \\
5 & 2.0 & \\
\hline 514 & 3.9 & 6.4
\end{tabular}




\section{Introduction}

This proposal is an outline of how Fermilab intends to construct and operate a System Test Facility for the SSC R\&D phase. It is a response to the specifications presented by the Systems Test Facility Task Force, chaired by $R$. Neal. It contains technical information, initial costs, operating costs, and schedules for proceeding with the System Test Facility at Fermilab.

There are two string tests involved in the STF.

1. A long string of magnets, eventually growing to one percent of the SSC length.

2. A short string of magnets, one half-cell in length, used primarily for life tests.

The long string of magnets is further divided (in time) into two phases.

1.1 Phase I - A short string of one cell (or even less) in length. The main purpose of Phase $I$ is to get started.

1.2 Phase II - The final length of one percent of the SSC. The transition between Phase I and Phase II will take place during CY 1986, probably in numerous steps. The cost estimates are broken into what is necessary to get Phase I operating, and the additional cost to get Phase II.

It is our expectation that the short string test (not to be confused with Phase I of the long string test) will be started sometime between the start of Phase I and the start of Phase II. The schedule for both the long string and short string is shown in Table $I$.

The descriptions and cost estimates, for each phase of the long string test, and for the short string test, are separated into:

1. Civil construction.

2. Cryogenics.

3. Power supplies and quench protection.

4. Controls and data collection.

5. Safety. 
E-2

\section{SYSTEM TEST FACILITY TIMETABLE}

DEC 85

Complete "Zero Dipole" runs

(All instrumentation cross calibrated)

(Baseline heat leak measured)

(Refrigerator controls stabilized)

Jan 86

Start installation one half cell string

Jan 87

Finish installation ten half cell string both aperatures

SHORT STRING FACILITY TIMETABLE

Dec $85^{\circ}$ Magnet type chosen

Mar 86 Detailed design of speciality components finished

July $86 \quad$ Magnets available short string; install

Oct 86-0ct 87 Run existing list of tests (Type B \& C)

$O R$

Oct 86-Apr 88 Run existing list of test (Type A) 


\section{Installation.}

7. Test Coordination (physicists and engineers conducting the tests).

\section{General Description}

\section{A. The Long String Test.}

Fermilab proposes to erect a head house and a 3500' "tunnel" along Kautz Road, extending from the E4 Refrigerator Building to Butterfield Road, as shown in Fig. 1.

The refrigeration will be supplied by a second satellite refrigerator installed at E4, and two existing compressors at $F \emptyset$. Initially, the power supply will be an extra Main Ring supply located at the E4 Service Building. For Phase II, a holding supply will have to be installed in the head house.

B. The Short Test String.

Fermilab proposes to install the short test string at Industrial Building $\# 1$, extending out the side of the building, as shown in Fig. 2. The refrigeration will be supplied by the MTF $1500 \mathrm{~W}$ refrigerator, and power supplies and controls will use the existing MTF system. Since the life test will require numerous magnetic measurement, in order to detect degradation of the magnets, it seemed advantageous to use the MTF system, which is keyed to magnetic measurements.

\section{Costs}

The costs for each fiscal year, 1985 through 1988, for the construction and operation of the test program are shown in Tables II and III, respectively. The manpower estimates for those years are shown in Table IV. The cost of the manpower is included in Tables II and III, assuming $\$ 60 \mathrm{~K}$ per year for professional staff, and $\$ 40 \mathrm{~K}$ per year for technicians. Each of the tables is divided into three parts, so that the total cost, and the costs for the long string and short string are shown separately.

\begin{tabular}{ll} 
TABLE & \multicolumn{2}{c}{ TITLE } \\
II A & Total Construction Cost (K\$) \\
II B & Long String Construction Cost (SK) \\
II C & Short String Construction Cost (SK) \\
III A & Total Operating Cost (\$K) \\
III B & Long String Operating Cost (\$K) \\
III C & Short String Operating Cost (\$K) \\
IV A & Total Manpower Estimate (M-YrS) \\
IV B & Long String Manpower Estimate (M-YrS) \\
IV C & Short String Manpower Estimate (M-YrS)
\end{tabular}

Details of the costs and manpower estimates are given in the chapters on the individual subsystems. 


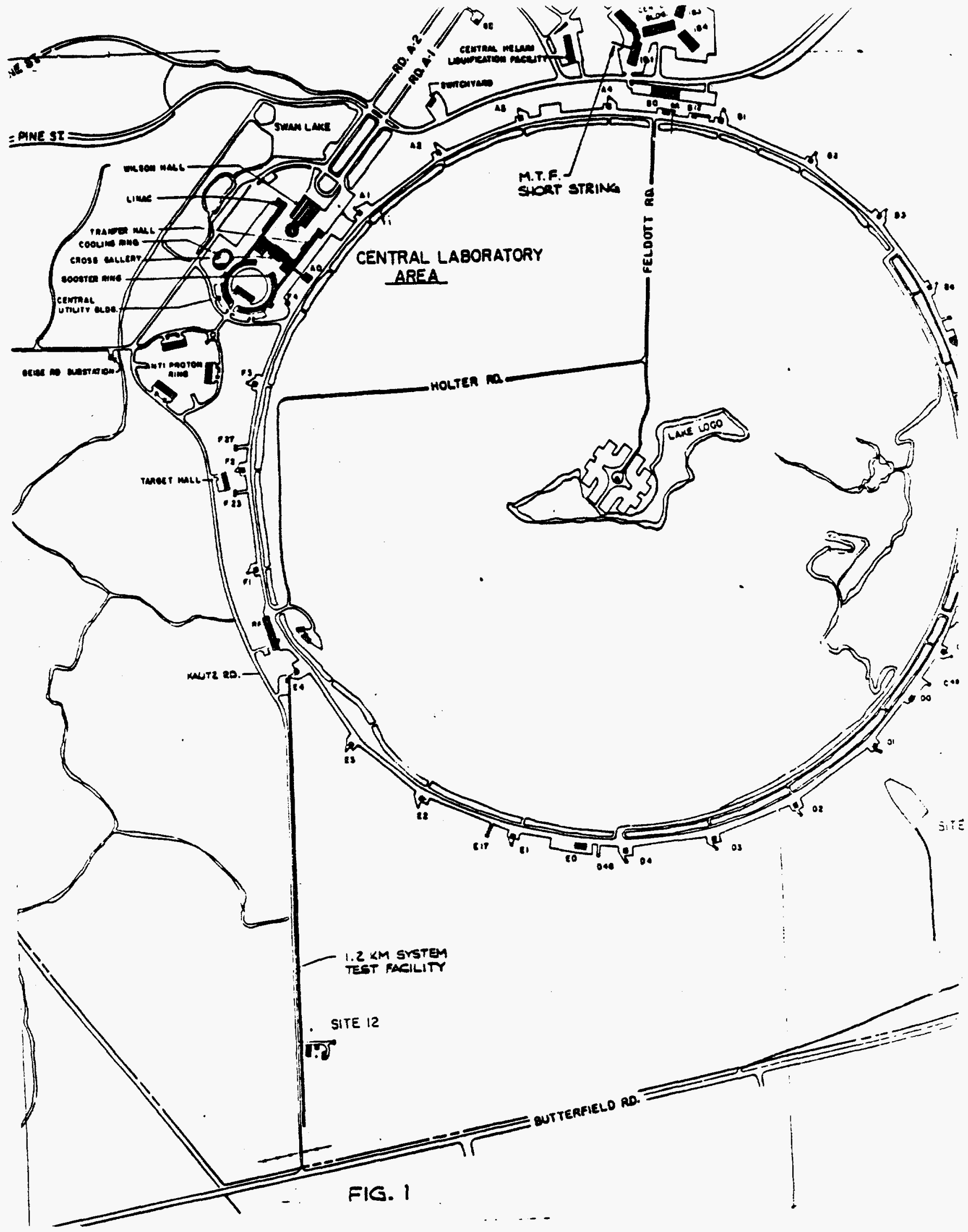


E-5

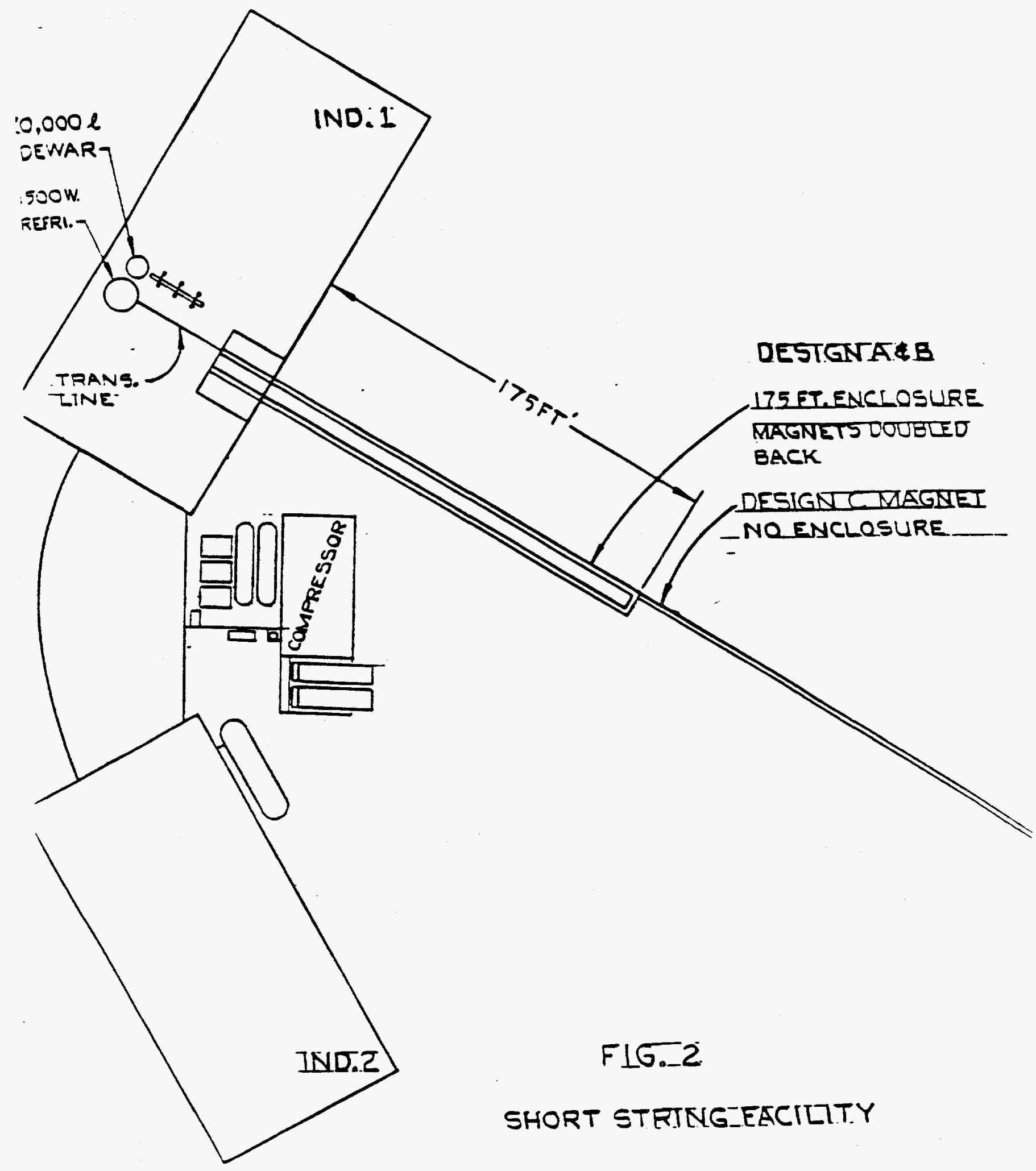




\begin{tabular}{|c|c|c|c|c|c|c|c|c|c|c|c|c|c|c|c|c|c|c|c|c|c|c|c|c|}
\hline & \multicolumn{6}{|c|}{ FY' 85} & \multicolumn{6}{|c|}{ FY ' 86} & \multicolumn{6}{|c|}{ FY' 87} & \multicolumn{6}{|c|}{ FY' 88} \\
\hline & \multicolumn{2}{|c|}{ TYPEA } & \multicolumn{2}{|c|}{ TrPE $B$} & \multicolumn{2}{|c|}{ TYPEC } & \multicolumn{2}{|c|}{ TYPEA } & \multicolumn{2}{|c|}{ TYPE B } & \multicolumn{2}{|c|}{ TrPEC } & \multicolumn{2}{|c|}{ TYPE A } & \multicolumn{2}{|c|}{ TYPE $\mathbf{B}$} & \multicolumn{2}{|c|}{ TYPE C } & \multicolumn{2}{|c|}{ TYPEA } & \multicolumn{2}{|c|}{ TYPE $B$} & \multicolumn{2}{|c|}{ TYPE C } \\
\hline & MBS & SWF & Mas & SWF & mas & SWF & Mas & swF & Mas & swF & MAS & $5 W F$ & Mas & SWF & MOS & SWF & Mas & swF & Mas & SWF & MAS & SWF & MBS & SWF \\
\hline $\begin{array}{l}\text { 1. CIVIL } \\
\text { CONSTRUCTION }\end{array}$ & 166 & & 166 & & 166 & & 542 & & 542 & & 534 & & & & & & 30 & & & & & & & \\
\hline 2. CAYOGENICS & 130 & 200 & 130 & 200 & 130 & 200 & 105 & 230 & 105 & 230 & 105 & 230 & & & & & & & & & & & & \\
\hline $\begin{array}{l}\text { 3. POWER SUPPLY } B \text { OPM } \\
\text { (SOFTWARE) }\end{array}$ & 106 & $\begin{array}{l}50 \\
30\end{array}$ & 122 & $\begin{array}{l}70 \\
30\end{array}$ & 106 & $\begin{array}{l}50 \\
30\end{array}$ & 199 & $\begin{array}{r}100 \\
60\end{array}$ & 299 & $\begin{array}{r}190 \\
60\end{array}$ & 560 & $\begin{array}{r}140 \\
60\end{array}$ & & & & & & & & & & & & \\
\hline $\begin{array}{l}\text { 4. CONTROLS AND DATA } \\
\text { COLLECTION } \\
\text { (SOFTWARE) }\end{array}$ & 82 & $\begin{array}{l}90 \\
60 \\
\end{array}$ & 82 & $\begin{array}{l}90 \\
60 \\
\end{array}$ & 82 & $\begin{array}{r}90 \\
60 \\
\end{array}$ & & 60 & & 60 & & 60 & & & & & & & & & & & & \\
\hline 5. SAFETY & 8 & 3 & 8 & 3 & 8 & 3 & 7 & 2 & 7 & 2 & 7 & 2 & & & & & & & & & & & & \\
\hline $\begin{array}{l}\text { 6. INSTALLLATION } \\
\text { MECHANICAL } \\
\text { ELECTRICAL }\end{array}$ & 25 & $\begin{array}{l}80 \\
40\end{array}$ & 25 & $\begin{array}{l}80 \\
40\end{array}$ & 50 & $\begin{array}{l}80 \\
40\end{array}$ & 125 & $\begin{array}{r}120 \\
60\end{array}$ & 125 & $\begin{array}{r}120 \\
60\end{array}$ & 200 & $\begin{array}{r}120 \\
60\end{array}$ & & & & & & & & & & & & \\
\hline 7. TEST COORDINATION & & 120 & & 120 & & 120 & & 30 & & 30 & & 30 & & & & & & & & & & & & \\
\hline B MISCELL ANEOUS & 25 & & 25 & & 25 & & 20 & & 20 & & 20 & & & & & & & & & & & & & \\
\hline 9. CRYOGENIC FLUIDS & 38 & & 20 & & 10 & & 3 & & 1 & & 1 & & & & & & & & & & & & & \\
\hline \multicolumn{25}{|l|}{10 POWER } \\
\hline 11 totals & 580 & 673 & 578 & 693 & 577 & 673 & 1001 & 662 & 1099 & 752 & 1427 & 702 & & & & & 30 & & & & & & & \\
\hline 12 TOTALS & \multicolumn{2}{|c|}{1253} & \multicolumn{2}{|c|}{1271} & \multicolumn{2}{|c|}{1250} & \multicolumn{2}{|c|}{1663} & \multicolumn{2}{|c|}{1851} & \multicolumn{2}{|c|}{2129} & & & & & \multicolumn{2}{|c|}{30} & & & & & & \\
\hline
\end{tabular}




\begin{tabular}{|c|c|c|c|c|c|c|c|c|c|c|c|c|c|c|c|c|c|c|c|c|c|c|c|c|}
\hline & \multicolumn{6}{|c|}{ FY'B5 } & \multicolumn{6}{|c|}{ FY '86 } & \multicolumn{6}{|c|}{ FY' 87} & \multicolumn{6}{|c|}{ FY ' 88} \\
\hline & \multicolumn{2}{|c|}{ TYPE A } & \multicolumn{2}{|c|}{ TYPE $B$} & \multicolumn{2}{|c|}{ TYPEC } & \multicolumn{2}{|c|}{ TYPE A } & \multicolumn{2}{|c|}{ TYPE $B$} & \multicolumn{2}{|c|}{ TYPEC } & \multicolumn{2}{|c|}{ TYPE A } & \multicolumn{2}{|c|}{ TYPE $B$} & \multicolumn{2}{|c|}{ TYPE C } & \multicolumn{2}{|c|}{ TYPE A } & \multicolumn{2}{|c|}{ TYPE $B$} & \multicolumn{2}{|c|}{ TYPE C } \\
\hline & $M B S$ & SWF & Mas & SWF & Mas & SWF & mas & SWF & Mas! & sWF & MBS & SWF & MAS & SNF & mas & SWF & Mas & SNF & Mas & $S H^{F}$ & 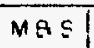 & $s W^{F}$ & MAS! & SWF \\
\hline $\begin{array}{l}\text { CIVIL } \\
\text { CONSTRUCTION }\end{array}$ & 166 & & 166 & & 166 & & 522 & & 522 & & 514 & & & & & & 30 & & & & & & & \\
\hline 2. CRYOGENICS & 35 & 100 & 35 & 100 & 35 & 100 & 100 & 110 & 100 & 110 & 100 & 110 & & & & & & & & & & & & \\
\hline $\begin{array}{l}\text { 3. POWER SUPPLY B OPM } \\
\text { (SOFTWARE) }\end{array}$ & 106 & $\begin{array}{l}50 \\
30\end{array}$ & 122 & $\begin{array}{l}70 \\
30\end{array}$ & 106 & $\begin{array}{l}50 \\
30\end{array}$ & 199 & $\begin{array}{l}50 \\
30\end{array}$ & 299 & $\begin{array}{r}140 \\
30\end{array}$ & 560 & $\begin{array}{l}90 \\
30\end{array}$ & & & & & & & & & & & & \\
\hline $\begin{array}{l}\text { 4. CONTROLS AND DATA } \\
\text { COLLECTION } \\
\text { (SOFTWARE) }\end{array}$ & 82 & $\begin{array}{l}90 \\
60 \\
\end{array}$ & 82 & $\begin{array}{l}90 \\
60 \\
\end{array}$ & 82 & $\begin{array}{l}90 \\
60 \\
\end{array}$ & & & & & & & & & & & & & & & & & & \\
\hline 5. SAFETY & 8 & 3 & 8 & 3 & 8 & 3 & 7 & 2 & 7 & 2 & 7 & 2 & & & & & & & & & & & & \\
\hline $\begin{array}{l}\text { 6. INSTALLATION } \\
- \text { - MECHANICAL } \\
\text { ELECTRICAL } \\
\end{array}$ & 25 & $\begin{array}{l}80 \\
40 \\
\end{array}$ & 25 & $\begin{array}{l}80 \\
40\end{array}$ & 50 & $\begin{array}{l}80 \\
40\end{array}$ & 100 & $\begin{array}{l}80 \\
40\end{array}$ & 100 & $\begin{array}{l}80 \\
40\end{array}$ & 150 & $\begin{array}{l}80 \\
40\end{array}$ & & & & & & & & & & & & \\
\hline 7. TEST COORDINATION & & 120 & & 120 & & 120 & & & & & & & & & & & & & & & & & & \\
\hline 8. miscellañous & 25 & & 25 & & 25 & & & & & & & & & & & & & & & & & & & \\
\hline 9. CRYOGENIC FLUIDS & 38 & & 20 & & 10 & & & & & & & & & & & & & & & & & & & \\
\hline \multicolumn{25}{|l|}{ IO POWER } \\
\hline 11. TOTALS & 485 & 573 & 483 & 593 & 482 & 573 & 928 & 312 & 1028 & 402 & 1331 & 352 & & & & & 30 & & & & & & & \\
\hline 12 TOTALS & \multicolumn{2}{|c|}{1058} & \multicolumn{2}{|c|}{1076} & \multicolumn{2}{|c|}{1055} & \multicolumn{2}{|c|}{1240} & \multicolumn{2}{|c|}{1430} & \multicolumn{2}{|c|}{1683} & & & & & \multicolumn{2}{|l|}{30} & & & & & \\
\hline & & & & & & & & $E$ & NOT & I UD & a A & & & & & & AND T & & & 98 & 250 & & & 68 \\
\hline
\end{tabular}




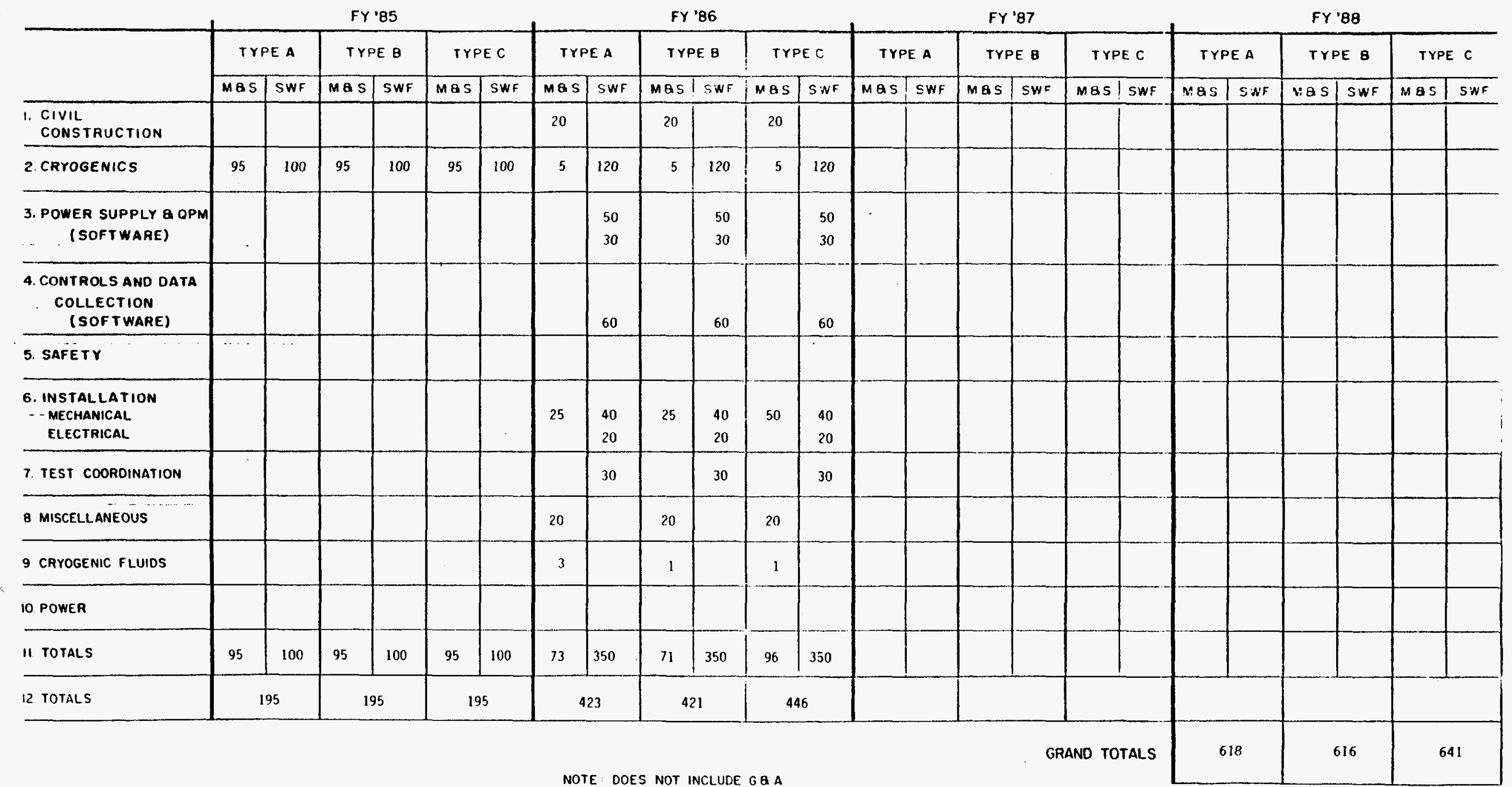




\section{Fermi III.A}

OPERATING COST (K\$) -- TOTAL

\begin{tabular}{|c|c|c|c|c|c|c|c|c|c|c|c|c|c|c|c|c|c|c|c|c|c|c|c|c|}
\hline & \multicolumn{6}{|c|}{ FY' 85} & \multicolumn{6}{|c|}{ FY' 86} & \multicolumn{6}{|c|}{ FY' 87} & \multicolumn{6}{|c|}{ FY ${ }^{\prime} 88$} \\
\hline & \multicolumn{2}{|c|}{ TYPE A } & \multicolumn{2}{|c|}{ TYPE $B$} & \multicolumn{2}{|c|}{ TYPEC } & \multirow{2}{*}{\multicolumn{2}{|c|}{\begin{tabular}{|l|l|}
\multicolumn{2}{|c}{ TYPEA } \\
MQS & SWF \\
\end{tabular}}} & \multicolumn{2}{|c|}{ TYPE B } & \multicolumn{2}{|c|}{ TYPE C } & \multicolumn{2}{|c|}{ TYPEA } & \multicolumn{2}{|c|}{ TYPE B } & \multicolumn{2}{|c|}{ TYPE C } & \multicolumn{2}{|c|}{ TYPEA } & \multicolumn{2}{|c|}{ TYPE $B$} & \multicolumn{2}{|c|}{ TYPE C } \\
\hline & \begin{tabular}{|l|} 
Mas \\
\end{tabular} & SWF & MOS & SWF & MAS & SWF & & & M85 & SWF & MAS & SWF & $M A S$ & SWF & $M \& S$ & SWF & MOS & SWF & MOS & SWF & $M \otimes S$ & SWF & $\ln \theta 5$ & SWF \\
\hline \multicolumn{25}{|l|}{$\begin{array}{l}\text { 1. CIVIL } \\
\text { CONSTRUCTION }\end{array}$} \\
\hline 2. CRYOGENICS & & & & & & & 22 & 120 & 22 & 120 & 22 & 120 & 100 & 430 & 100 & 430 & 100 & 430 & 72 & 320 & 45 & 210 & 45 & 210 \\
\hline $\begin{array}{l}\text { 3. POWER SUPPLY B OPM } \\
\text { (SOFTWARE) }\end{array}$ & & & & & & & 30 & $\begin{array}{r}130 \\
90\end{array}$ & 30 & $\begin{array}{r}130 \\
90\end{array}$ & 60 & $\begin{array}{r}130 \\
90\end{array}$ & 70 & $\begin{array}{r}170 \\
90\end{array}$ & 70 & $\begin{array}{r}170 \\
90\end{array}$ & 140 & $\begin{array}{r}170 \\
90\end{array}$ & 60 & $\begin{array}{r}100 \\
60\end{array}$ & 50 & $\begin{array}{r}100 \\
60\end{array}$ & 100 & $\begin{array}{r}100 \\
60\end{array}$ \\
\hline \multicolumn{25}{|l|}{$\begin{array}{l}\text { 4. CONTAOLS AND DATA } \\
\text { COLLECTION } \\
\text { (SOFTWARE) }\end{array}$} \\
\hline \multicolumn{25}{|l|}{ 5. SAFETY } \\
\hline $\begin{array}{l}\text { 6. INSTALLLATION } \\
\text { MECHANICAL } \\
\text { ELECTRICAL } \\
\end{array}$ & & & & & & & & $\begin{array}{l}80 \\
40 \\
\end{array}$ & & $\begin{array}{l}80 \\
40 \\
\end{array}$ & & $\begin{array}{l}80 \\
40\end{array}$ & & $\begin{array}{l}80 \\
40\end{array}$ & & $\begin{array}{l}80 \\
40\end{array}$ & & $\begin{array}{l}80 \\
40 \\
\end{array}$ & & $\begin{array}{l}80 \\
40\end{array}$ & & $\begin{array}{l}80 \\
40\end{array}$ & & $\begin{array}{l}80 \\
40\end{array}$ \\
\hline 7. TEST COORDINATION & & & & & & & & 180 & & 180 & & 180 & & 210 & & 210 & & 210 & & 195 & & 180 & & 180 \\
\hline 8. MISCELLLANEOUS & & & & & & & 25 & & 25 & & 25 & & 90 & & 90 & & 90 & & 70 & & 50 & & 50 & \\
\hline 9. CRYOGENIC FLUIDS & & & & & & & 35 & & 35 & & 35 & & 825 & & 375 & & 330 & & 120 & & 70 & & 70 & \\
\hline 10 POWER & & & & & & & 75 & & 75 & & 75 & & 625 & & 475 & & 475 & & 238 & & 150 & & 150 & \\
\hline 1. TOTALS & & & & & & & 187 & 640 & 187 & 640 & 217 & 640 & 1710 & 1050 & 1110 & 1050 & 1135 & 1050 & 560 & 810 & 365 & 670 & 415 & 670 \\
\hline 12 totals & & & & & & & \multicolumn{2}{|c|}{827} & \multicolumn{2}{|c|}{827} & \multicolumn{2}{|c|}{857} & \multicolumn{2}{|c|}{2760} & \multicolumn{2}{|c|}{2160} & \multicolumn{2}{|c|}{2185} & \multicolumn{2}{|c|}{1370} & \multicolumn{2}{|c|}{1035} & \multicolumn{2}{|c|}{1085} \\
\hline & & & & & & & NOT & $E:$ & $\mathbf{N}$ & NCL & $3 \mathrm{~A}$ & & & & & & D & CALS & & 4957 & & 1022 & & 127 \\
\hline
\end{tabular}




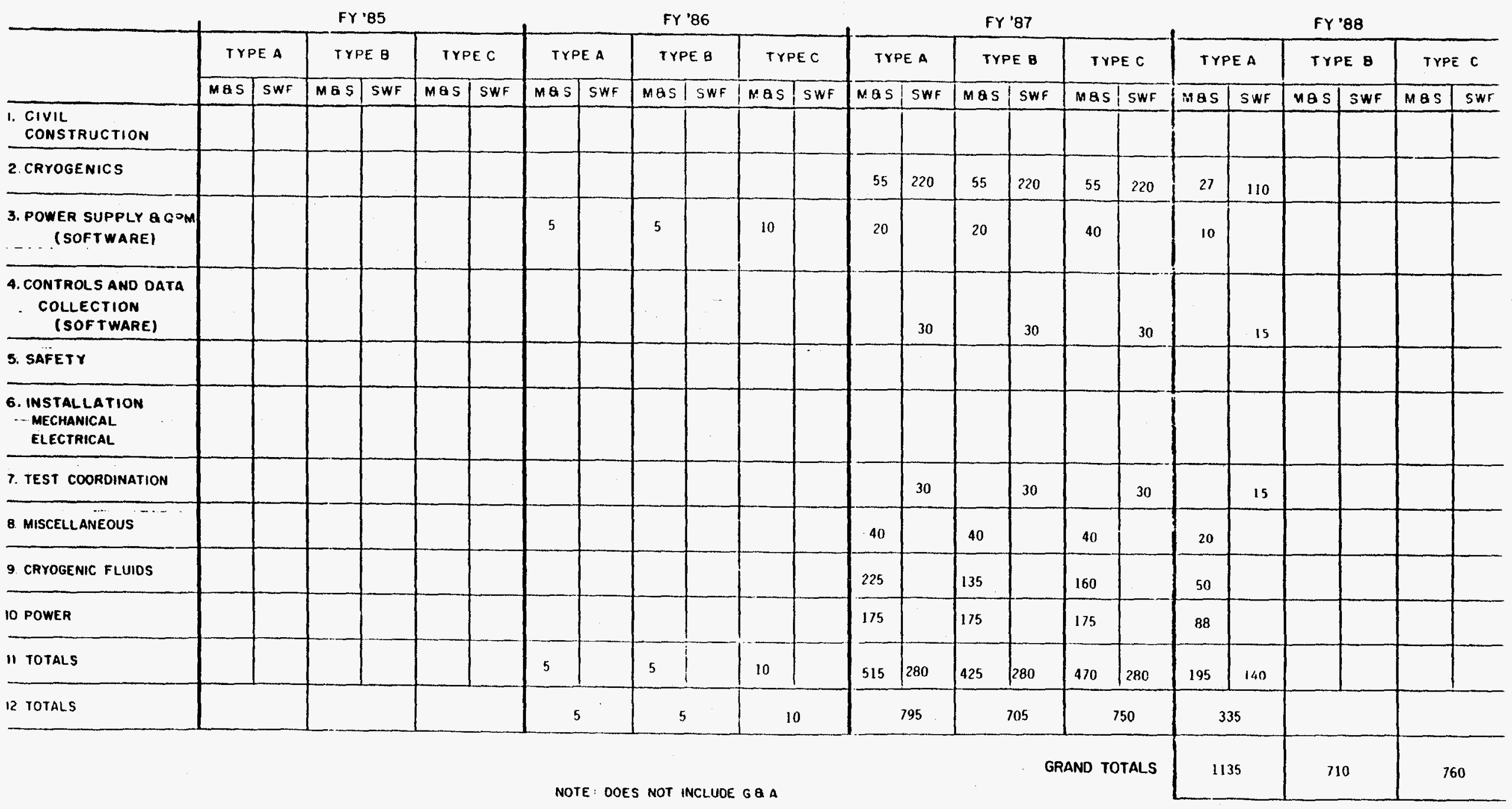

NOTE: The FYBB costs for type $A$ are required since both the cooldown mass and quench recovery double the ref $x$ igeration loads. We have costed 


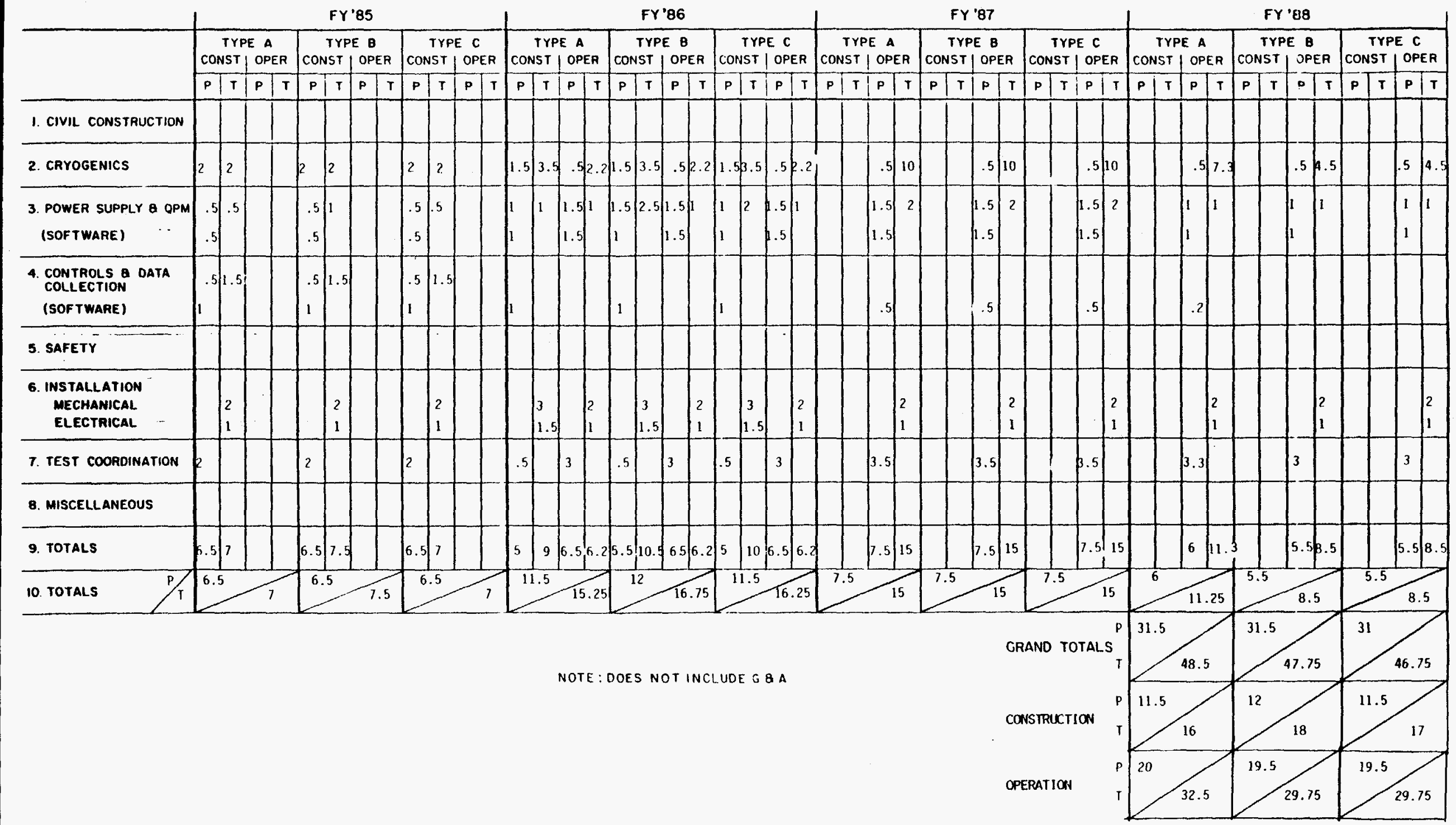




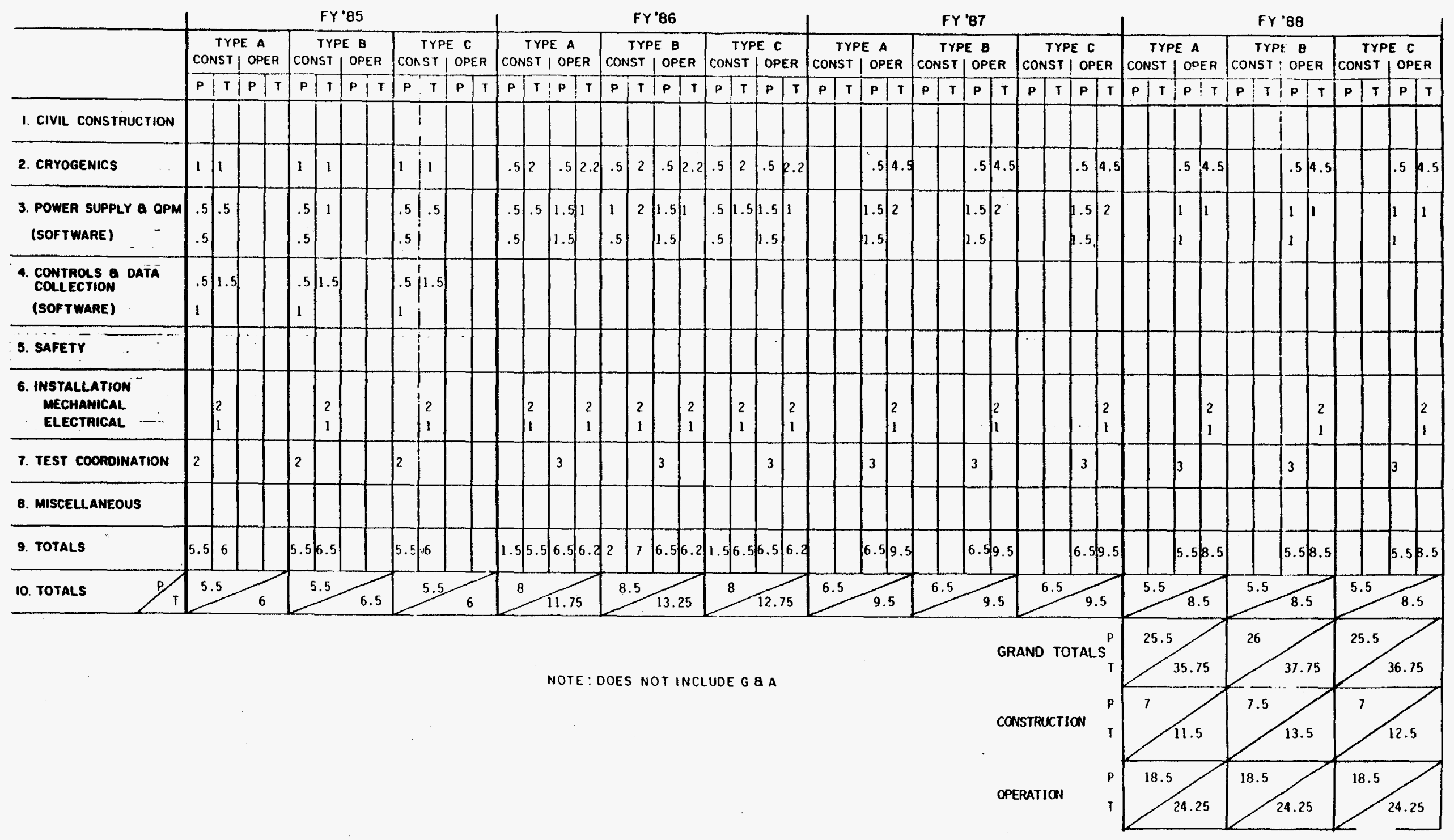




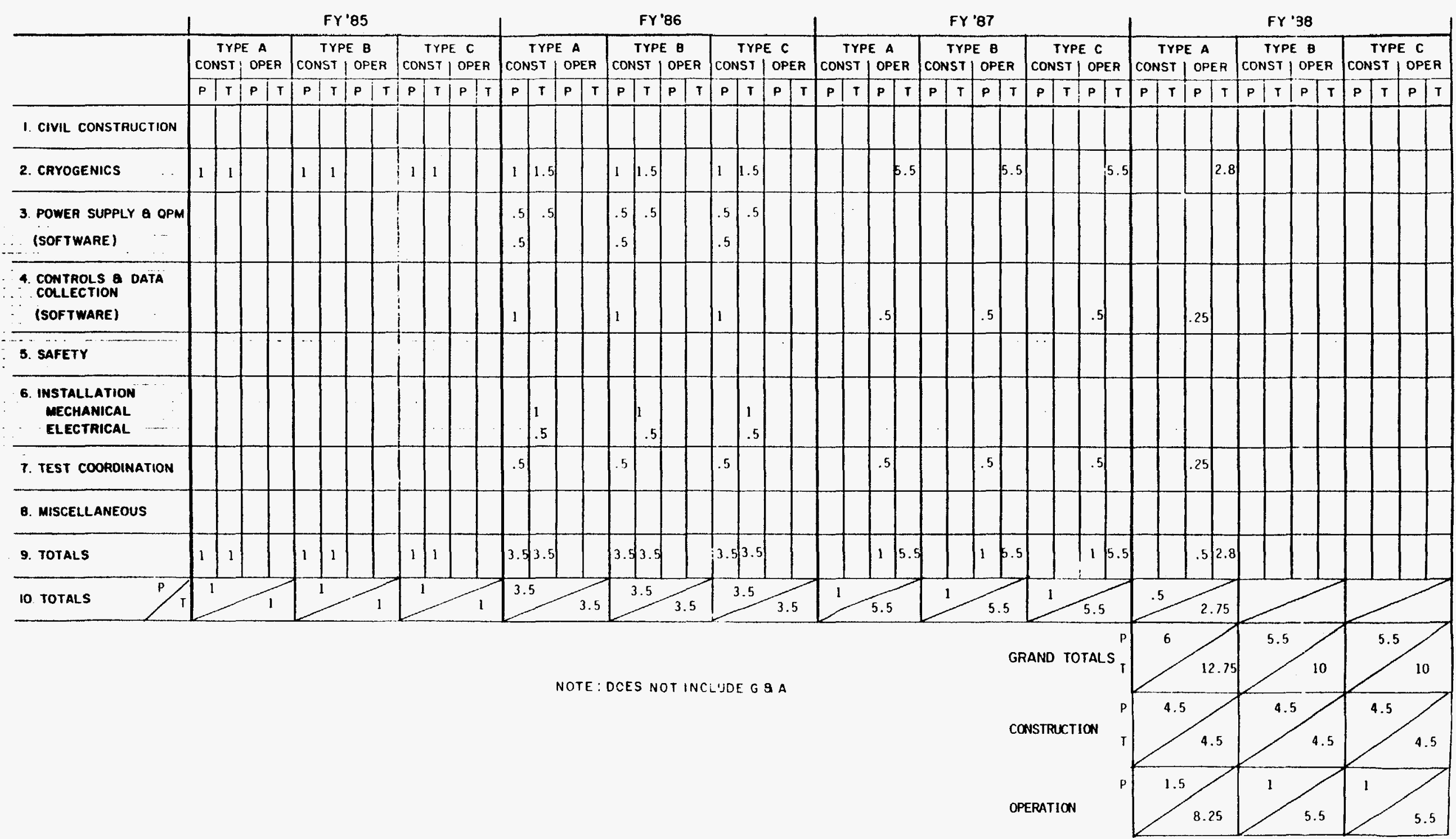




\section{CIVIL CONSTRUCTION}

\subsection{The Long String.}

The long string will be emplaced along Kautz Road from the vicinity of the RF Building ( $F D$ ) south for 1.2 kilometers. The string will be connected to the Tevatron cryogenic system at the E4 Refrigerator Building. This building will be extended by 16' towards the outside of the ring to house the connections and an additional satellite cold box. Adjacent to this extension and at grade level will be a $40^{\prime} \times 40^{\prime}$ pre-engineered building that serves for power supply and tech space along with the head end of the string. An existing spare 500 kVA substation will supply technical and house power for the facility. The substation will be connected to Feeder 45 at the E4 manhole.

1.1.1 For Phase I a $10^{\prime} \times 700^{\prime}$ long pole building with concrete slab floor will extend from the head house to and along Kautz Road. Magnet power for the Phase I testing will be bused across the berm from a pulsed supply in the E4 Service Building. $3000^{\prime}$ to Butterfield Road by erecting an extension of Phase I pole building on a $10^{\prime}$ wide slab of concrete on the existing road bed. A holding power supply will be installed in the head 
E-16

house and connected in series with the bus from E4. The holding power supply will require a second 500 kVA substation connected, again at manhole E4, to Feeder 48. For type $C$ magnets a 2 MVA substation would be required in place of the 500 kVA substation.

\subsection{The Short String.}

The short string test will involve a small amount of civil construction. It will utilize the existing IB-I (MTF) with adjacent asphalted hardstand as necessary, and a $10^{\prime}$ wide enclosure, 175' long. 


\section{PHASE I}

E4 Refrigerator Building - 16' $\times 16^{\prime}$ extension

$\$ 17 \mathrm{~K}$

Head House $-40^{\prime} \times 40^{\prime}$ pre-engineered @ $\$ 20 / \mathrm{ft}^{2}$

$32 \mathrm{~K}$

"Tunnel" - $10^{\prime} \times 700^{\prime}$ pole building e $\$ 7 / \mathrm{ft}^{2}$

$49 \mathrm{~K}$

Concrete slab $\$ 3 / f t^{2}$

$21 \mathrm{~K}$

Insulation @ $\$ 18 /$ l.f.

$6 \mathrm{~K}$

HVAC

$12 \mathrm{~K}$

Electrical (1ighting, utility power, tech power)

(a) $50 \mathrm{~kW} / \mathrm{cell}$

$14 \mathrm{~K}$

$15 \mathrm{~K}$

$500 \mathrm{kVA}$ * substation installation (incl. 480V)

Subtotal Phase I

PHASE II

"Tunne1" extension - $10^{\prime} \times 3250^{\prime}$ pole building @ $\$ 7 / \mathrm{ft}^{2}$ $\$ 227 \mathrm{~K}$

Concrete slab \& $\$ 3 / \mathrm{ft}^{2}$

$97 \mathrm{~K}$

Insulation

$58 \mathrm{~K}$

HVAC

$52 \mathrm{~K}$

Electrica1

$65 \mathrm{~K}$

$500 \mathrm{kVA}^{*}$ substation - procurement

$8 \mathrm{~K}$

insta1lation

$15 \mathrm{~K}$

Subtotal Phase II

*For Type $C$ a 2 MVA substation would be required. This would increase procurement cost by $\$ 22 \mathrm{~K}$. 


\section{INTRODUCTION}

System Test Facility is a test set up for the magnets and related systems. We propose to attach it to the Tevatron Cryogenic and Control Systems at the location of the "E4" satelitite refrigerator. We would move a spare satellite to the "E4" location and pipe it to two compressors in the "FO" building. (Figures 1,2 and 7)

This permits us to use a small refrigerator to supply the steady state refrigeration, while for transient conditions we can use the $4500 \mathrm{l} / \mathrm{hr}$ CHL together with its $60,000 \mathrm{l}$ of stored 1 iquid and 35,000 l equivalent of gas storage. The refrigerator will be run unattended except when a detailed cryogenic experiment is in progress. Logging, monitoring, alarms, and quench recovery will be provided by the esisting Tevatron control system.

\section{CONFIGURATION}

The interface between the "ER" satellite refrigerator and magnet string for the four different configuration is given in Eigures 3 through 6 . The output of the wet expander is subcooled and supplies the magnet single phase. In three of the four configurations a circulating pump supplements the flow. Iiquid helium is also supplied from $\mathrm{CHL}$, up to $100 \mathrm{~g} / \mathrm{sec}$ $(3000 \mathrm{l} / \mathrm{hr})$; this is injected in one of two bayonets devending on the configuration and running mode.

For the $3.5^{\circ} \mathrm{K}$ operation we will use a cold centrifugal compressor on the return gas; depending on the configuration it pumps either $3.5 \mathrm{~K}$ or $15 \mathrm{~K}$ gas. The refrigerator has three refurn gas lines to cover the temperature range $2 \varnothing-8^{\circ}-25^{\circ}$ $60^{\circ}$.

Figure 7 shows the interfaces between the Tevatron anci the "ER" refrigeratcr. We will isolate the suction and discharce header between "FO" compressors \#2 and 3 . The FO \#1 and 2 compressors are connected directly to the "ER" refrigerator which is $280 \mathrm{ft}$. away. The buffer for the system is the Tevatron 3 in. high pressure header backed up by the CHL tank farm.

For quench recovery the kickback valve (FOEVDL) position will be transmitted to the "BO" $\mu \mathrm{P}$ which does the Tevatron inventory control. An additional important aspect of the quench recovery is the back pressure regulator on the $10^{\circ} \mathrm{K}$ line, which permits the quench header in the magnets to store the $20^{\circ} \mathrm{K}$ gas until it can be used by the refrigerator. For the 
System Test. Facility - Cryo. Description (cont.)

injtial operation (one half cell) we plan to use the 5000 l $20^{\circ}$ buffer tank which will later be part of the short string facility. The design goal is that no helium is vented for half cell quenches. For full string quenches it appears that there is no reasonable way to save the gas; we will assume it is all vented. NOTE: Longest operating quench in the Tevatron was $300 \mathrm{~m}$.

\section{CAPACITY}

The capacity of the system is given in Tables I and II and in Figure 8. The refrigeration capacity is sufficiently large that initial operation would be in "standalone" mode; as the string is lengthened we will switch to the higher capacity modes. The cooldown capacity has the same flexability. By combining the CHL dewar and excess CHL capacity we can trivially provide the design "B" specification of $100 \mathrm{~g} / \mathrm{sec}$ below $20^{\circ} \mathrm{K}$ (actually $5 \mathrm{~K}$ ). For design " $\mathrm{A}$ " we can provide 150. $\mathrm{g} / \mathrm{sec}$ at $30^{\circ} \mathrm{K}$ by adding an auxiliary $\mathrm{N}_{2}$ heat exchanger. Figure 8 shows the operating schedule of the Tevatron and CHI, as well as the steady state cooldown flow rates available. Table II shows the excess CHL capacity as a function of operating modes.

\section{TIMETABLE}

Table II gives the timetable for the Long string. The first quarter of FY86 will be test operation of the system with the half cell cooldown in January 86 . The string is expected to grow until about January 87 when it reaches full length. 


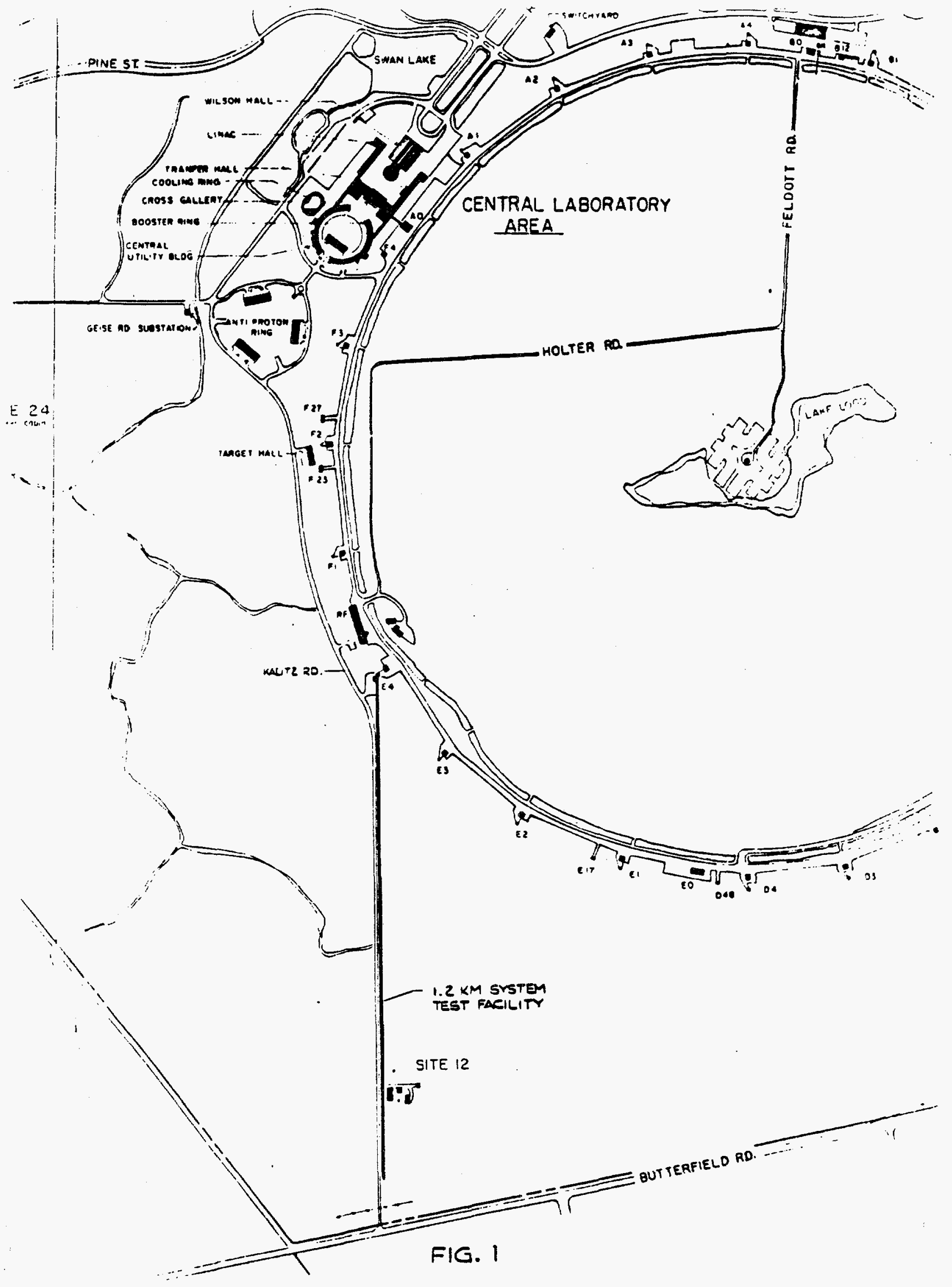




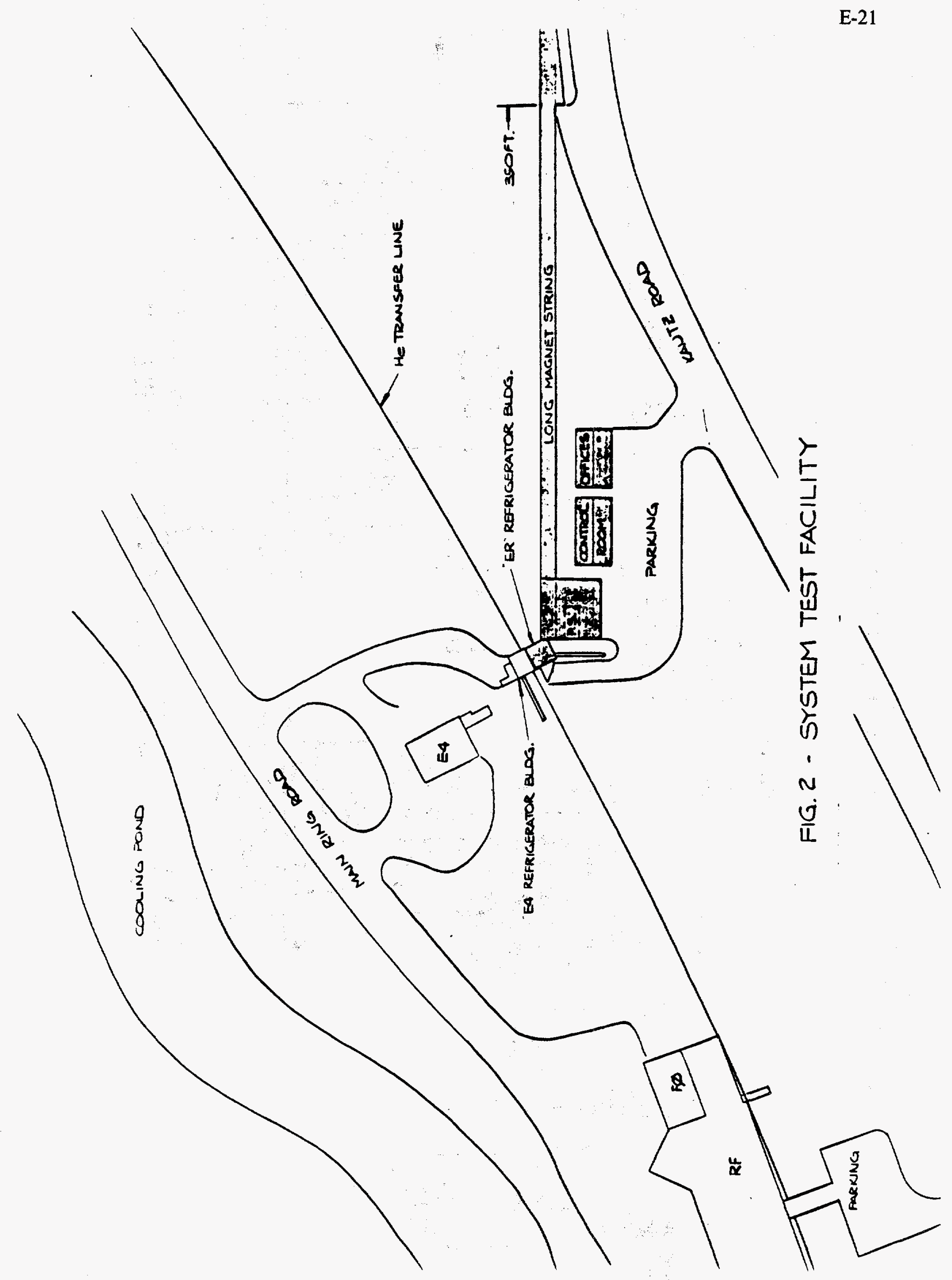




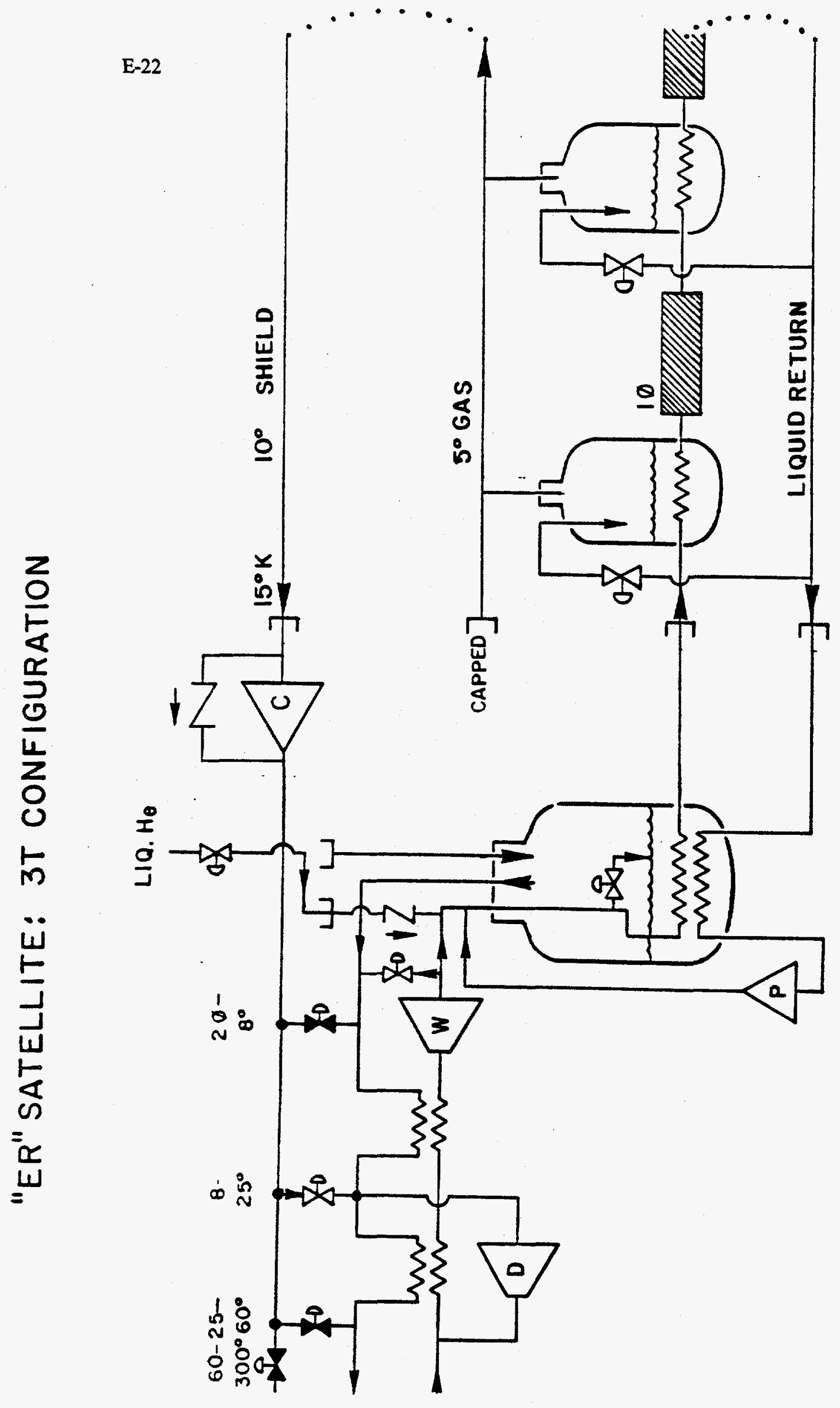

$m$
$\frac{0}{4}$ 


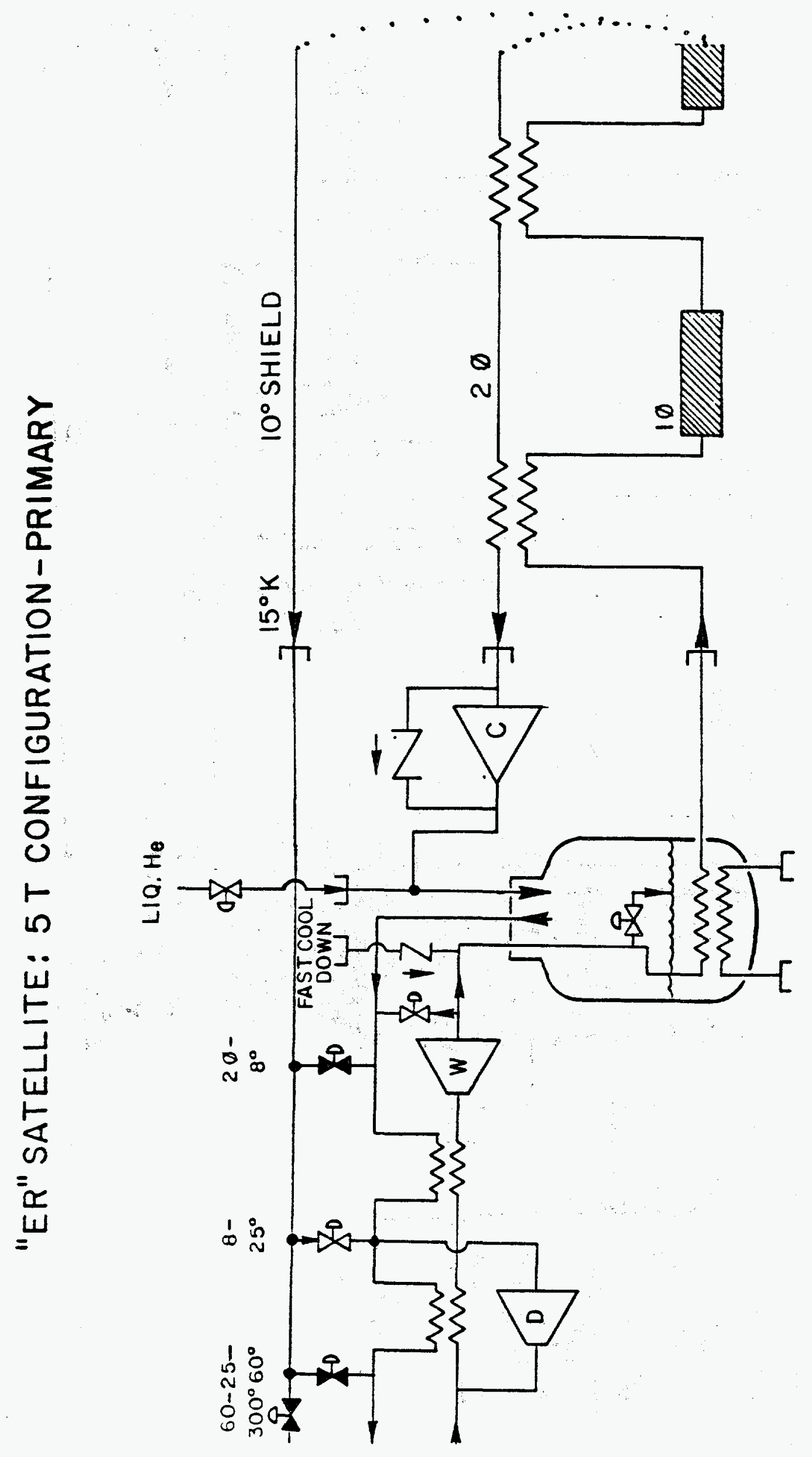

E-23

8
$\frac{0}{4}$ 


\section{"ER" SATELLITE: 5 T CONFIgURATION-ALTERnATE}

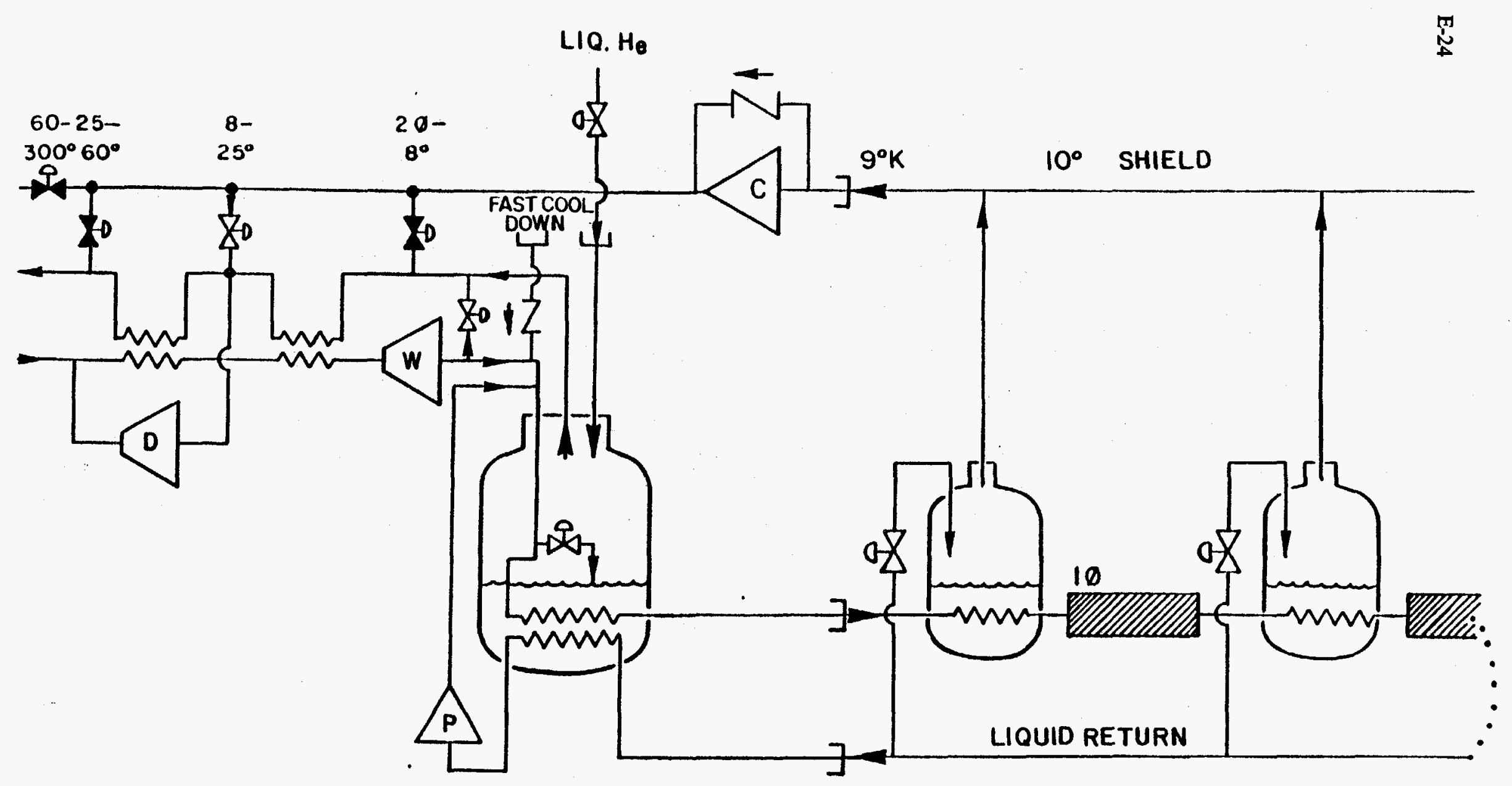




\section{"ER" SATELLITE: $6 \frac{1}{2}$ T CONFIGURATION}

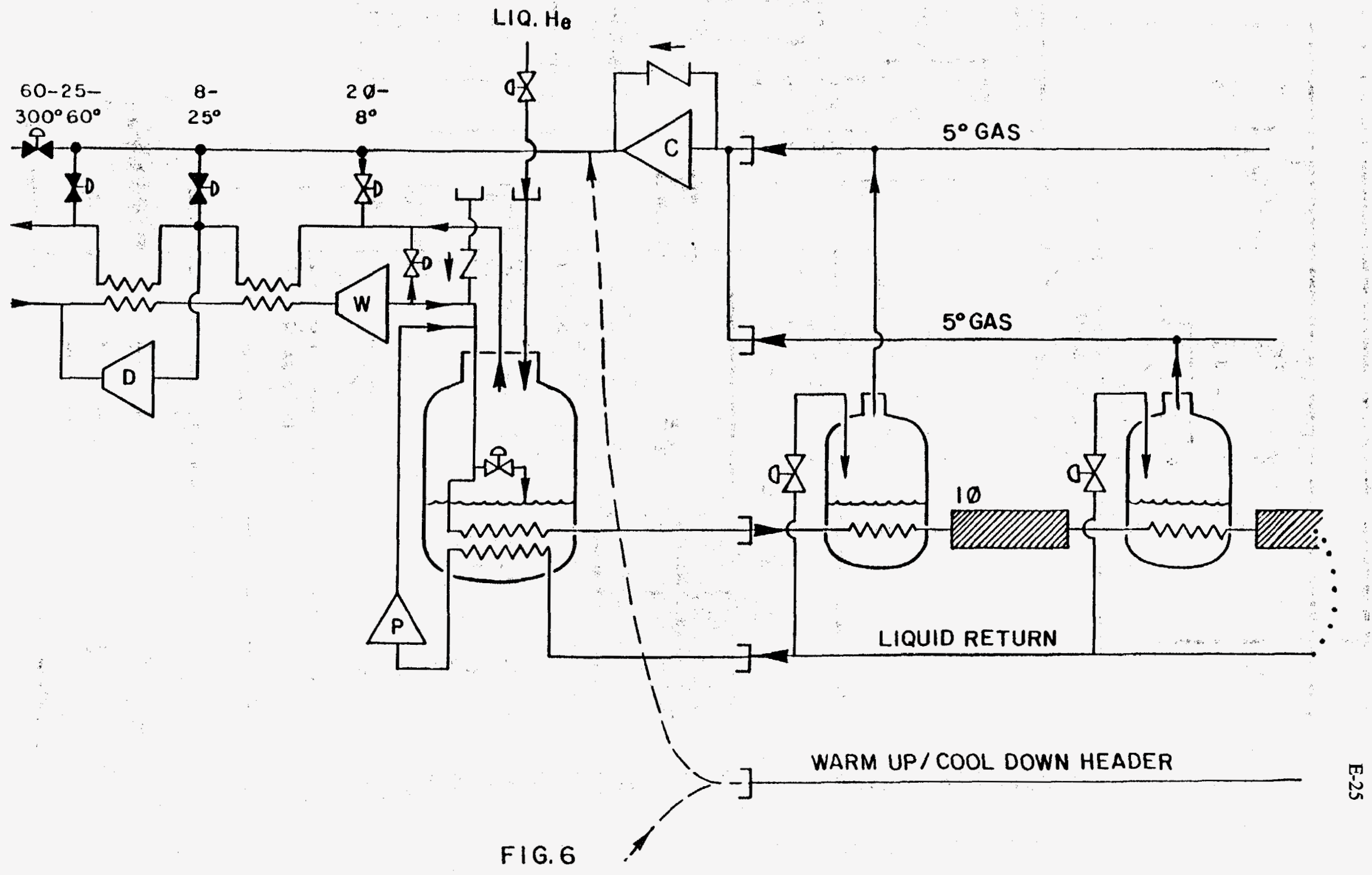


$\frac{2}{5}$

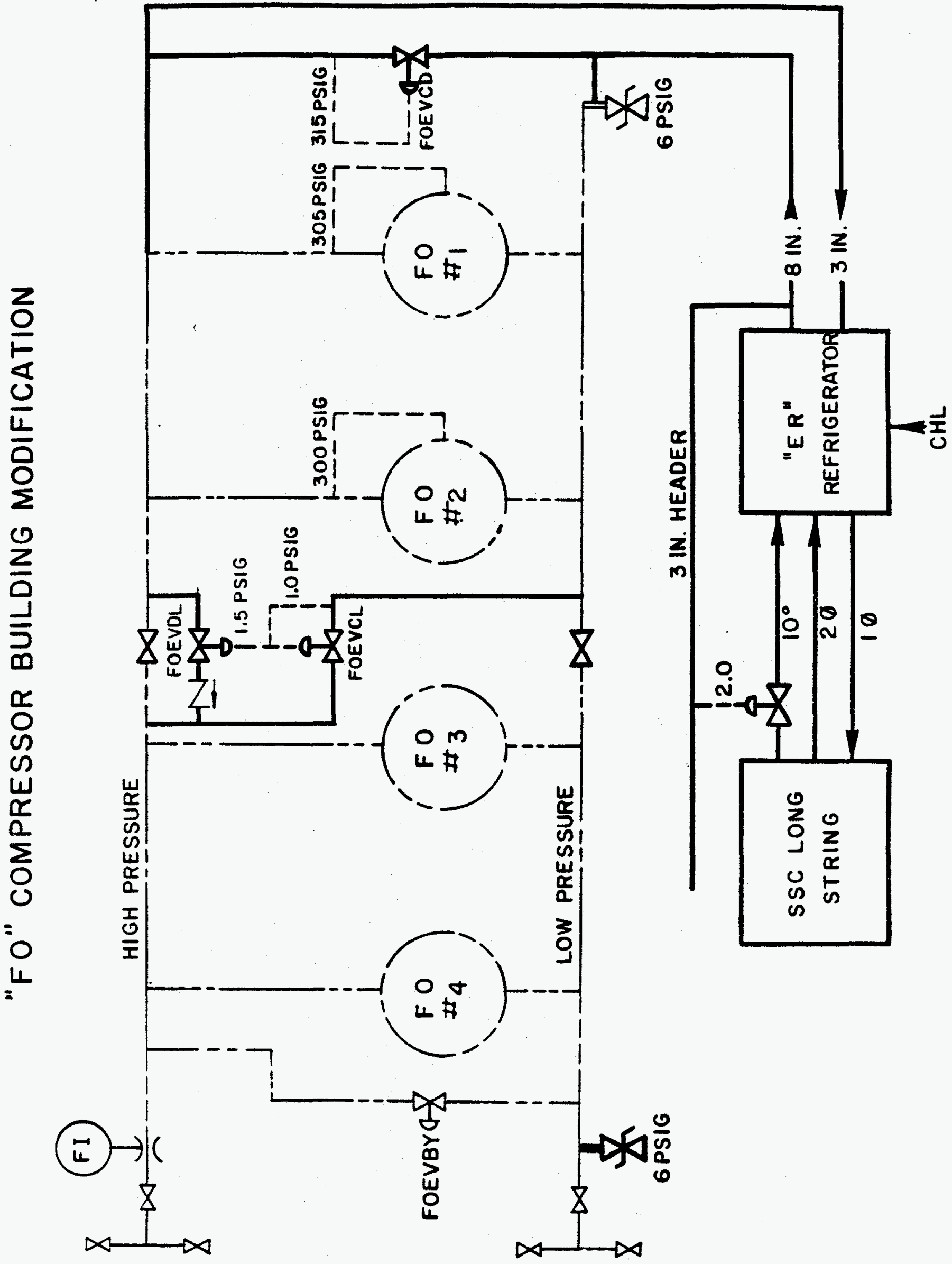

$\sim$
$\frac{0}{4}$ 


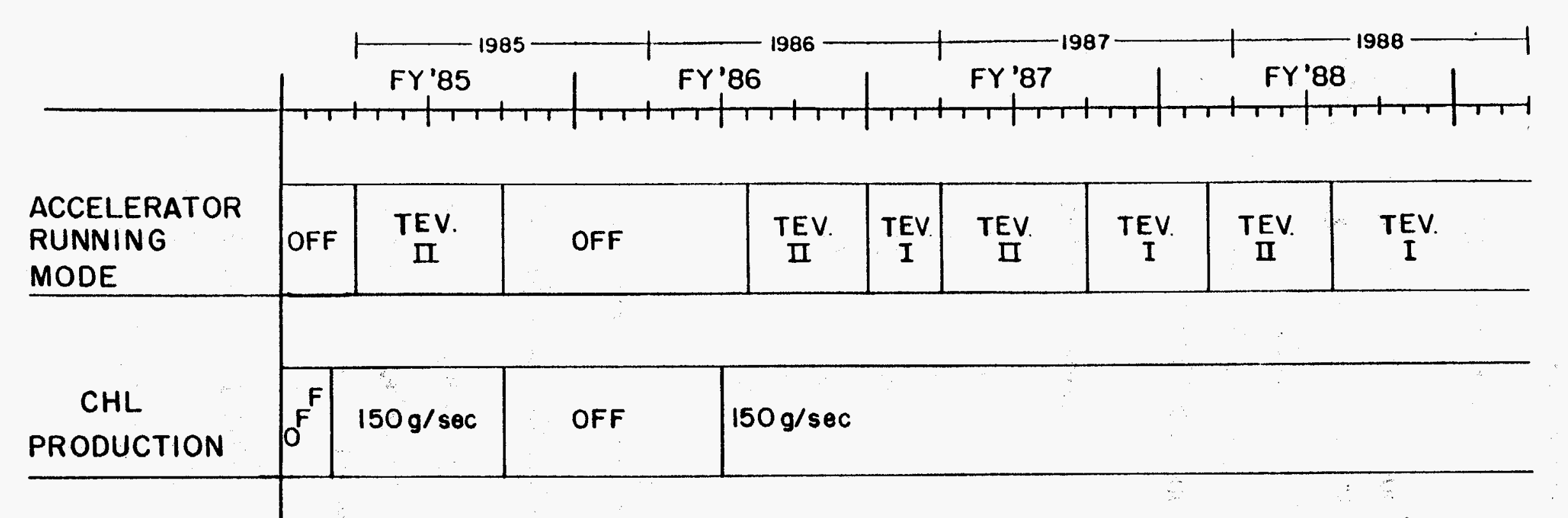

STEADY STATE COOLDOWN CAPACITY (WITHOUT USING DEWAR)

RING MODE / COOLDOWN CAPACITY

\begin{tabular}{|}
\begin{tabular}{c|c|c|c|c|c|c|c|c|c|c|c|}
\hline $\begin{array}{c}\text { SATELLITE } \\
\text { NA }\end{array}$ & NAF & OFF & 6 & SAT. & SAT. & SAT. & SAT. & SAT. & SAT. \\
\hline & 16 & 66 & 16 & 66 & 16 & 66 \\
\hline
\end{tabular} \\
LIMITED CHL MODE 350 RPM & 36 & 86 & 36 & 86 \\
\hline
\end{tabular}

LIMITED CHL MODE 800 RPM

\begin{tabular}{|l|l|l|l|}
\hline 75 & 115 & 75 & 115 \\
\hline
\end{tabular}


"Stand Alone" (One compressor)

$500 \mathrm{~W}+$

$375 W+$

"Satellite" (One compressor)

"Saver" ( 1.25 compressors)

"Full Capacity" (Two compressors)

$\begin{array}{rc}500 \mathrm{~W}+ & 25 \ell / \mathrm{hr} \\ 375 \mathrm{~W}+ & 50 / / \mathrm{hr} \\ 1000 \mathrm{~W}+ & \mathrm{CHL} \\ 1250 \mathrm{~W}+ & \mathrm{CHL} \\ >1500 \mathrm{~W}+ & \mathrm{CHL}\end{array}$

$g / \sec$

COOLDOWN MODES

4

6

25

75

"Full Capacity"

Using CHL Dewar

"Full Capacity"

100

Ring in "Limited CHL Mode"

Nominal Magnet Specification (omit sync, rad. and AC Heating)

5T One Section $(\sim 1 \mathrm{~km})$

5T Two Sections $(\sim 2 \mathrm{~km})$

5T Cooldown

$6.5 T \quad 0.9$ Kilometer ( $1 \%)$

$6.5 T \quad 1.2$ Killometer $(1.33 \%)$

3T $\quad 1.2$ Kilometer $(.8 \%)$
$212 W$

$425 W$

$100 \mathrm{~g} / \mathrm{sec}$

$429 W$

$572 W$

$340 \mathrm{~W}$
$40 \ell$ hr

$24 \mathrm{l} / \mathrm{hr}$

$48 \mathrm{l} / \mathrm{hr}$

$58 \mathrm{lihr}$

$35 \mathrm{e} / \mathrm{ir}$ 


\section{EXCESS CHL CAPACITY}

\section{REFRIGERATION MODES}

TeV II Operation

"Satellite"

"Limited CHL Mode - 350 rpm" (Spring 84)

"Limited CHL Mode - 800 rpm"
CAPACITY $(g / s e c)$

$\sim 10$

30

$\sim 75$

TeV I Operation

"Satell i te"

$\therefore 60$

"Limited CHL Mode - 350 rpm"

$\therefore 80$

"Limited CHL Mode - 800 rpm"

$-115$

"Stand Alone Refrigeration"

$-130$ 
E-30

\section{SYSTEM TEST FACILITY TIMETABLE}

DEC 85

Complete "Zero Dipole" runs

(All instrumentation cross cal ibrated)

(Basel ine heat leak measured)

(Refrigerator controls stabilized)

Jan 86

Start installation one half cell string

Jan 87

Finish installation ten half cell string both aperatures

\section{SHORT STRING FACILITY TIMETABLE}

Dec 85

Magnet type chosen

Mar 86

Detalled design of speciality components finished

July 86

Magnets available short string; install

Oct $86-0$ ct 87 Run existing list of tests (Type B \& C)

$O R$

Oct $86-A p r ~ 88$ Run existing list of test (Type A) 


\section{TESTING PHASES}

From a Cryogenic standpoint, in addition to the Phase I and II Testing there are two equally important phases which need to be discussed to get the proper prospective on the Phase I and II Testing.

\section{Pre-Phase I}

It is critical that preliminary checks be made that the magnets are compatable with the SSC Project. Rather than measuring just heat leak we need to determine a more fundamental quantity, the product of heat leak and flow impeciance, which determines the answer to the questions: 1) "Can the magnets be cooled in a finite time?" 2) "Can the magnets be operated below $4.5^{\circ} \mathrm{K}$ ?" As soon as possible we need to test a $100 \mathrm{M}$ string of magnets, even if these components are electric rejects (shorted turns, ground faults, vaporized coil, etc.)

If the answer to one of the above is no, it means a major civil or magnet redesign; the only options being:

1. Increased number of refrigerator buildings.

2. Increase magnet flow apertures

3. Decrease magnet heat leak

\section{Phase I}

Table IV provides the list of cryogenic tests for Phase I. Items \#1 and 2 will have preliminary results from Pre-phase I; Phase I will provide more accurate answers and a check that a design "improvement" error has not been made. Items \# 2 thrcugh 6 will receive preliminary answers in Phase I.

As soon as one half cell of good magnets exist, Phase I electrical testing will start, nominally January 86 . The configuration is given by Figures 3 through 6 , except that the circulating pump and cold compressor are omitted. The specification for both is given by Table $V$. While the pump is one of our dry expanders modified to pump helium, it is not needed because the string is so short. (The pump is interchangeable with those used for the CHL or the short string.)

The cold compressor will not be available until the latter part of Phase I since we cannot order it until the magnet design is chosen. We believe this is consistant with the current schedules; if the schedule should change we could procure a small cold compressor for initial operation with the possibility of having to buy a larger one later, or we probably can use the circulating pump with minor modifications. 
FERMILAB CRYOGENIC TEST GOALS (PHASE I AND EARLIER)

1. Five "one day" warm ups and cooldowns

Magnet stresses and bowing

Pressure Drops in magnets and piping

2. String Heat Load

3. System stability vs flow rate and percent liquid

4. Effect of ramp on refrigeration system

5. String quenching

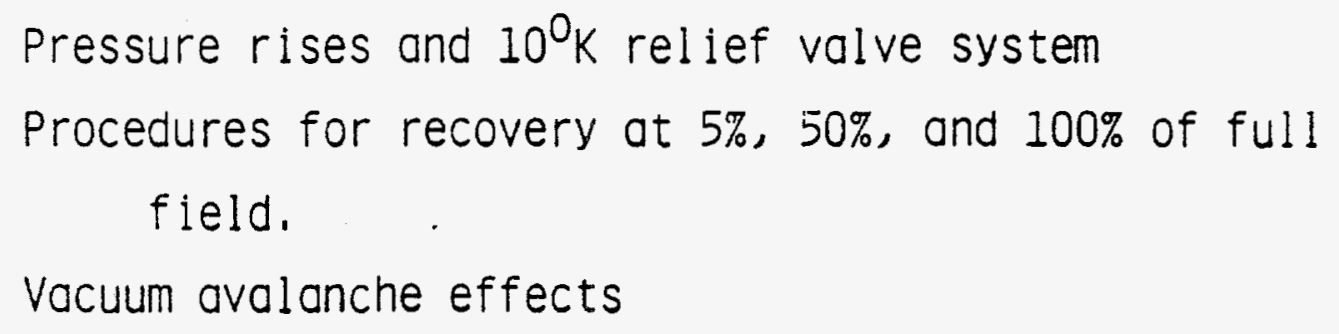

6. Failure modes

$10^{\circ} \mathrm{K}$ relief stuck closed

Power failure

He and $\mathrm{N}_{2}$ ruptures

Insulating vacuum loss 
Phase: II

For Phase II testing of the full string we will use the pump and the final cold compressor. The "ER" refrigerator will be operated in "Satellite" mode.

During the latter part of Phase II FY87/88 there will be some yet undefinable Cryogenic Tests needed to specify the refrigerator.

Tunnel Testing

The final phase of Cryogenic Testing is not part of the $R \& D$ program but is part of the construction program. In FY89 or 90 the real refrigerator will be hooked to $4 \mathrm{~km}$ strings of magnets in the SSC Tunnel. This will be the first system test of the refrigerator magnet combination, including vertical legs. Any problems discovered in this phase can be solved by modifying the interface or the refrigerator and therefore are not as critical.

Any problems that could affect the magnet design would have been found during Pre-Phase I and Phase I. The timing of tunnel testing is such that no matter how major a problem is found it can be corrected without affecting the SSC schedule. 


\section{"ER" SATELLITE PUMP AND COLD COMPRESSOR}

LIQUID HELIUM PUMP (Modified CTI dry expander)
Full speed
$7500 \ell / \mathrm{hr}$
$(\sim 250 \mathrm{~g} / \mathrm{sec})$
Idling
$375 \ell / h r$
$(\sim 121 / 2 \quad \mathrm{~g} / \mathrm{sec})$

Maximum output pressure 20. atm

Minimum input pressure $1.4 \mathrm{~atm}$

COLD GAS COMPRESSOR FOR SUBATMOSPHERIC OPERATION (must be procured)

1. Not required for initial testing

2. Specification

Pressures: $P$ in $1 / 2 \mathrm{~atm} \quad P$ out $11 / 2 \mathrm{~atm}$

THROUGHPUT:

Design A: $\quad 3.56^{\circ} \mathrm{K}, \quad-34 \mathrm{~g} / \mathrm{sec} \quad-F=4, \quad \ell / \mathrm{sec}$

Design $\mathrm{B}: \quad 3.56^{\circ} \mathrm{K}, \quad-10 \mathrm{~g} / \mathrm{sec} \quad-F=1.2 \mathrm{l} / \mathrm{sec}$

Design C: $15.00^{\circ} \mathrm{K}, \quad-10 \mathrm{~g} / \mathrm{sec} \quad-F=6.5 \ell / \mathrm{sec}$

3. Since the size of the cold compressor varies by a factor of eight, the cold compressor must be ordered after the magnet style is chosen and a preliminary heat load is measured for the half cell. 


\section{CRYOGENIC MANPOWER}

The largest cryogenic manpower components are the efforts to install and commission the refrigeration system and the manpower for the initial operation ( $\sim$ one year). Therefore we will use the "BR" Satellite Refrigerator (our $R$ \& D test unit) which is operational and is currently being set up to study $2 \varnothing$ flow. After the test we will either move the "BR" coldbox to "ER" or use one of our other spares. Therefore these efforts are very small and consist of the interface construction and installation, the weekly rotating machinery maintenance, and moving the refrigerator.

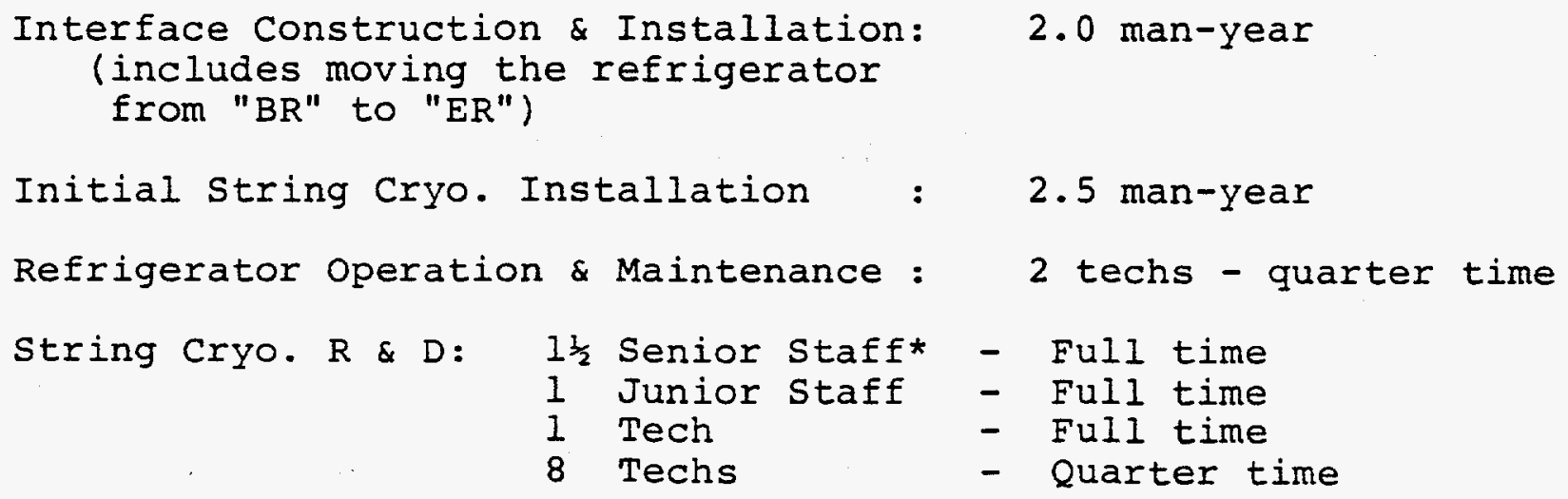

Total manpower: $1 / 1$ FY85

$2 / 4 \frac{1}{4}$ FY 86 *

$1 \frac{1}{2} / 4 \frac{1}{2} \quad F y 87 *$

$1 \frac{1}{2} / 4 \frac{1}{2}$ FY 88 *

* NOTE: One Senior Staff member is charged in monetary tables as one of the three Test coordinators. 
E-36

Long

OPERATING COST (Design "B")

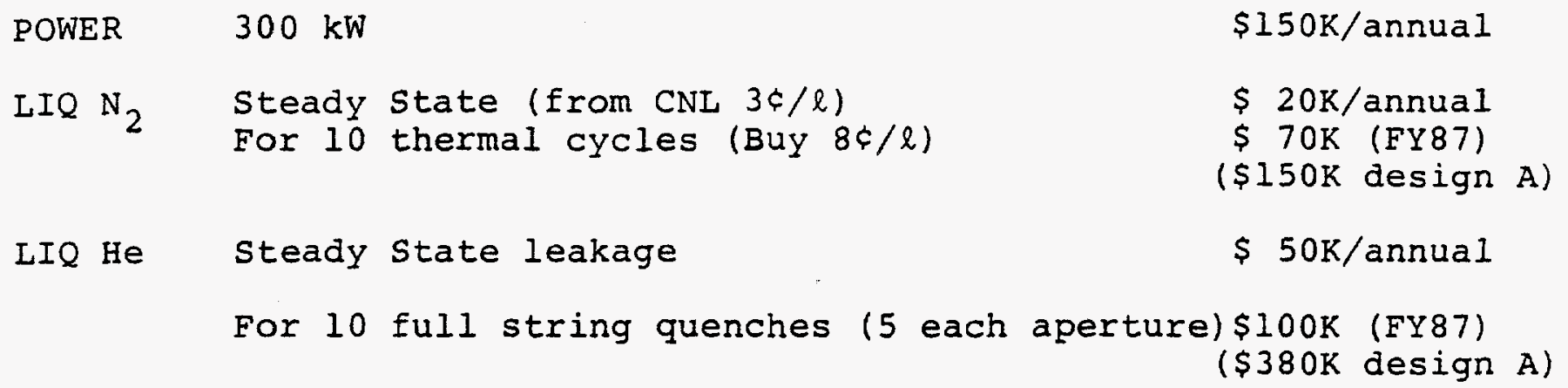

MATERIAL COST FOR OPERATION

Initial Cryo Charge

$\$ 20 K$ (FY85)

"ER" Piping Components

$\$ 10 \mathrm{~K}$ (FY85)

"ER" Interface Components (U-tubes, subcoolers etc.) $\$ 20 \mathrm{~K}$ (FY85)

"ER" Expander Upgrade (high lift cams, check valves, etc)

$\$ 5 K(F Y 85)$

cold compressor

$\$ 100 \mathrm{~K} \quad(F Y 86)$

Misc Nut and Bolts

$\$ 45 \mathrm{~K}$ annual

\begin{tabular}{|c|c|c|c|c|c|}
\hline BUDGET & LABOR & & MATERIAL & OPERATING & TOTAL \\
\hline FY85 & $\$ 100 \mathrm{~K}$ & $(1 / 1)$ & $55 \mathrm{~K}$ & OK & $\$ 155 \mathrm{~K}$ \\
\hline FY86 & $\$ 230 \mathrm{~K}$ & $\left(1 / 4 \frac{1}{4}\right)$ & $122 \mathrm{~K}$ & $110 \mathrm{~K}$ & $\$ 462 \mathrm{~K}$ \\
\hline FY87 & $\$ 210 \mathrm{~K}$ & $\left(\frac{1}{2} / 4 \frac{1}{2}\right)$ & $45 \mathrm{~K}$ & $540 \mathrm{~K}$ & $\$ 795 \mathrm{~K}$ \\
\hline \multirow[t]{2}{*}{ FY88 } & $\$ 210 \mathrm{~K}$ & $\left(\frac{1}{2} / 4 \frac{1}{2}\right)$ & $45 \mathrm{~K}$ & $220 \mathrm{~K}$ & $\$ 475 \mathrm{~K}$ \\
\hline & & & & & $\$ 1887 \mathrm{~K}$ \\
\hline \multicolumn{2}{|l|}{ Professional: } & \multicolumn{2}{|c|}{$\$ 60 \mathrm{~K} / \mathrm{man}$ year } & & \\
\hline Tech & $:$ & $\$ 40 \mathrm{~K} / \mathrm{I}$ & year & & \\
\hline
\end{tabular}


Short

SHORT STRING FACILITY - CRYOGENIC DESCRIPTION

\section{INTRODUCTION}

The short string is primarily electrical and cryogenic stress testing of a small number of magnets. Except from a cryogenic standpoint is is a smaller effort than the long string. Because of the 100 thermal cycles and 500 half cell quench requirement it cannot be simply connected to the Tevatron system. For this proposal we have assumed the requirement that the testing be relatively independent; this requires a major cryogenic design and construction effort.

We propose to attach it to the Magnet Test Facility 1500 watt refrigerator. This facility will be operating testing Tevatron and SSC Design "B" magnets. It contains six magnet testing outputs; we will tee the short string off the dewar and coldbox i.e. in parallel to the six outputs (Fig. I).

\section{CONF IGURATION}

The M.T.F. has facilities for two $60 . \mathrm{g} / \mathrm{sec}$ liquid helium pumps in the main dewar which provides a pumped circulation flow. In addition there are two additional return lines: 1) A $300^{\circ} \mathrm{K}$ cooldown line and 2) a vacuum jacketed quench recovery line, which would be used as a $10^{\circ} \mathrm{K}$ sinield return.

A $20^{\circ}$ buffer tank to store the quench gas for about one half hour is required for all three magnet types. It would need to be nitrogen shielded and vacuum jacketed with a pressure rating of 10 atm minimum with a volume of about 50002. Figures 2, 3 , and 4 are primarily schematics of the magnets connected to the M.T.F.

\section{CAPACITY}

The 1500 watt refrigerator capacity has been measured as 1950 watts or $350 \mathrm{l} / \mathrm{hr}$. The refrigerator is two orders of magnitude larger than the steady state heat load, but is closely matched to the transient heat loads in Table I. At the $20 \%$ level the refrigerator can produce in excess of $3 \mathrm{~kW}$. 
E-38

Cryogenic Description (cont.)

\section{TIME TABLE}

Table II gives the nominal time table for the short string test. For type $B$ and $C$ magnets, the test program as currently specified will take one year and use half the M.T.F. capacity (average); we assume that the testing will be done in FY87. For type A magnets because of the doubled cooldown mass and quench recovery energy we have allocated one and a half years with the test running into the first half of FY88. 


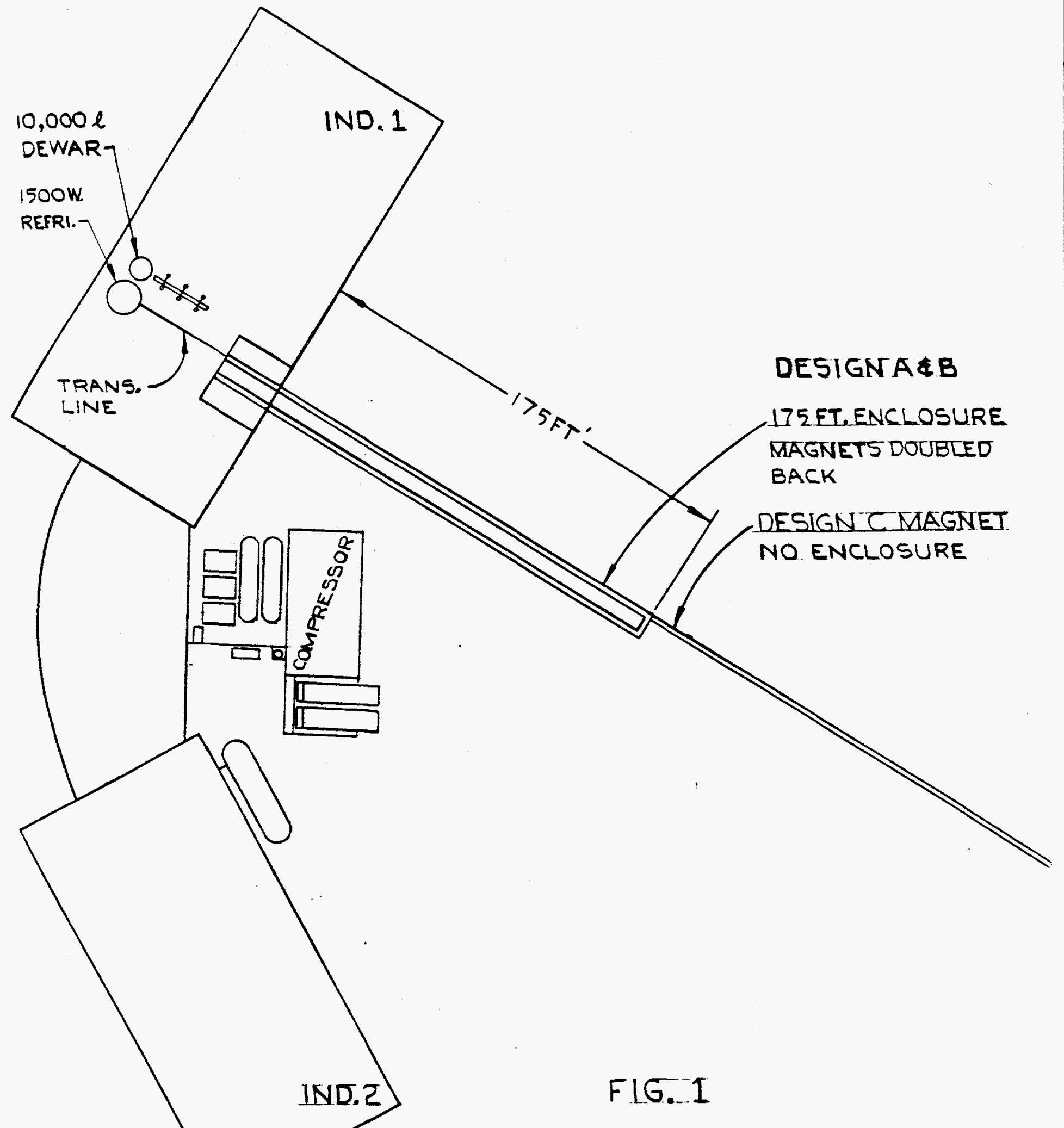

SHORT STRING FACILITY 


\section{M.T.F. SHORT STRING : 3 T CONFIGURATION}

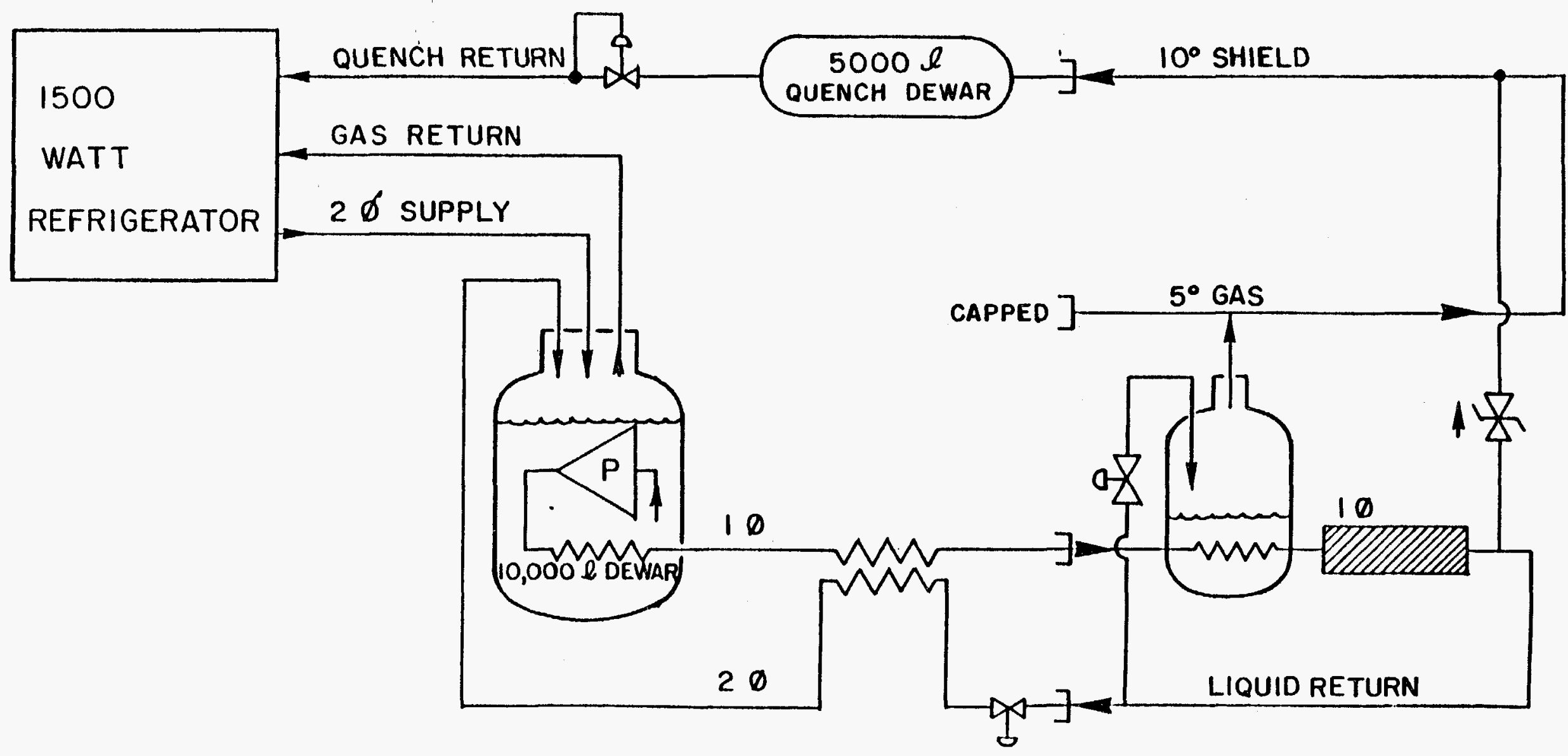

FIG. 2 


\section{M.T.F. SHORT STRING : $5 \mathrm{~T}$ CONFIGURATION}

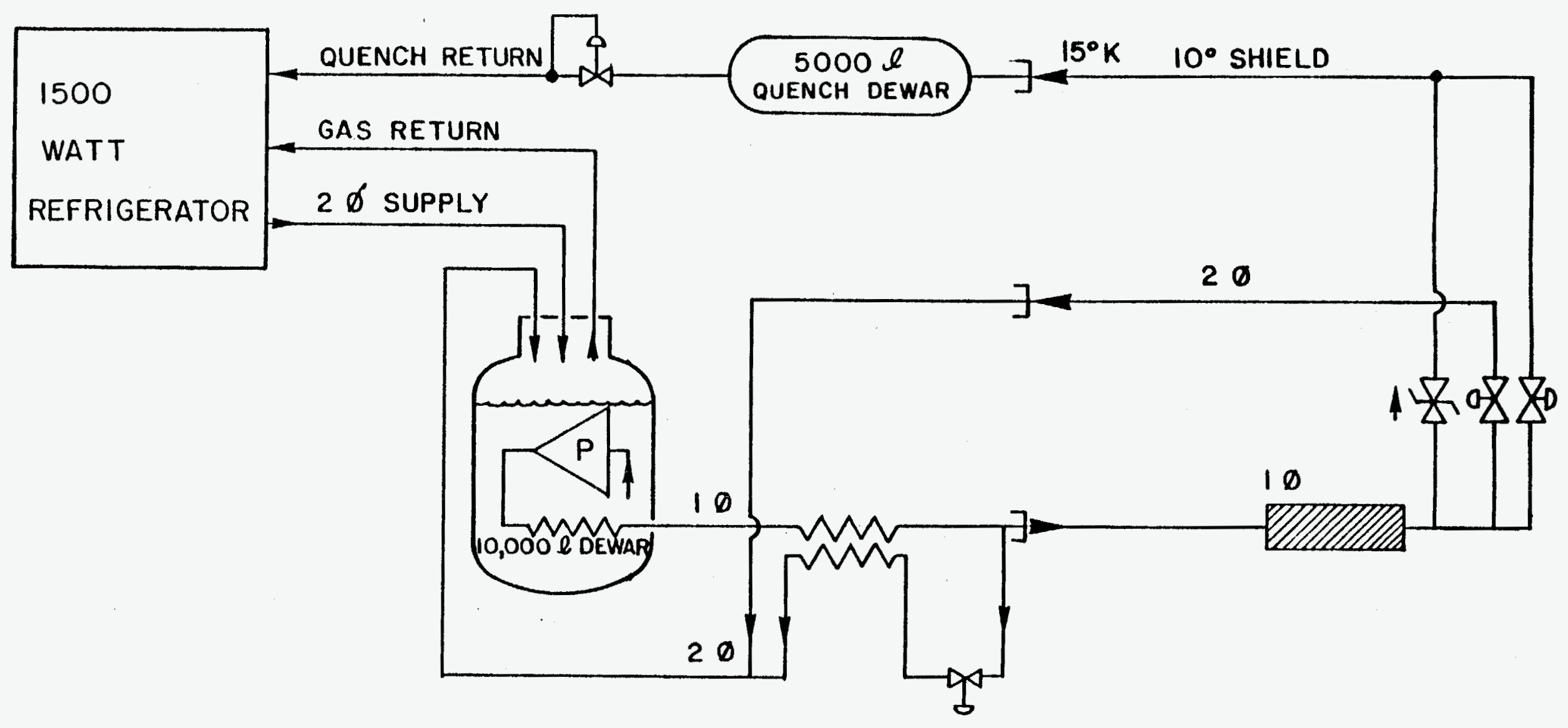

FIG. 3 
M.T.F. SHORT STRING : $6 \frac{1}{2} T$ CONFIGURATION

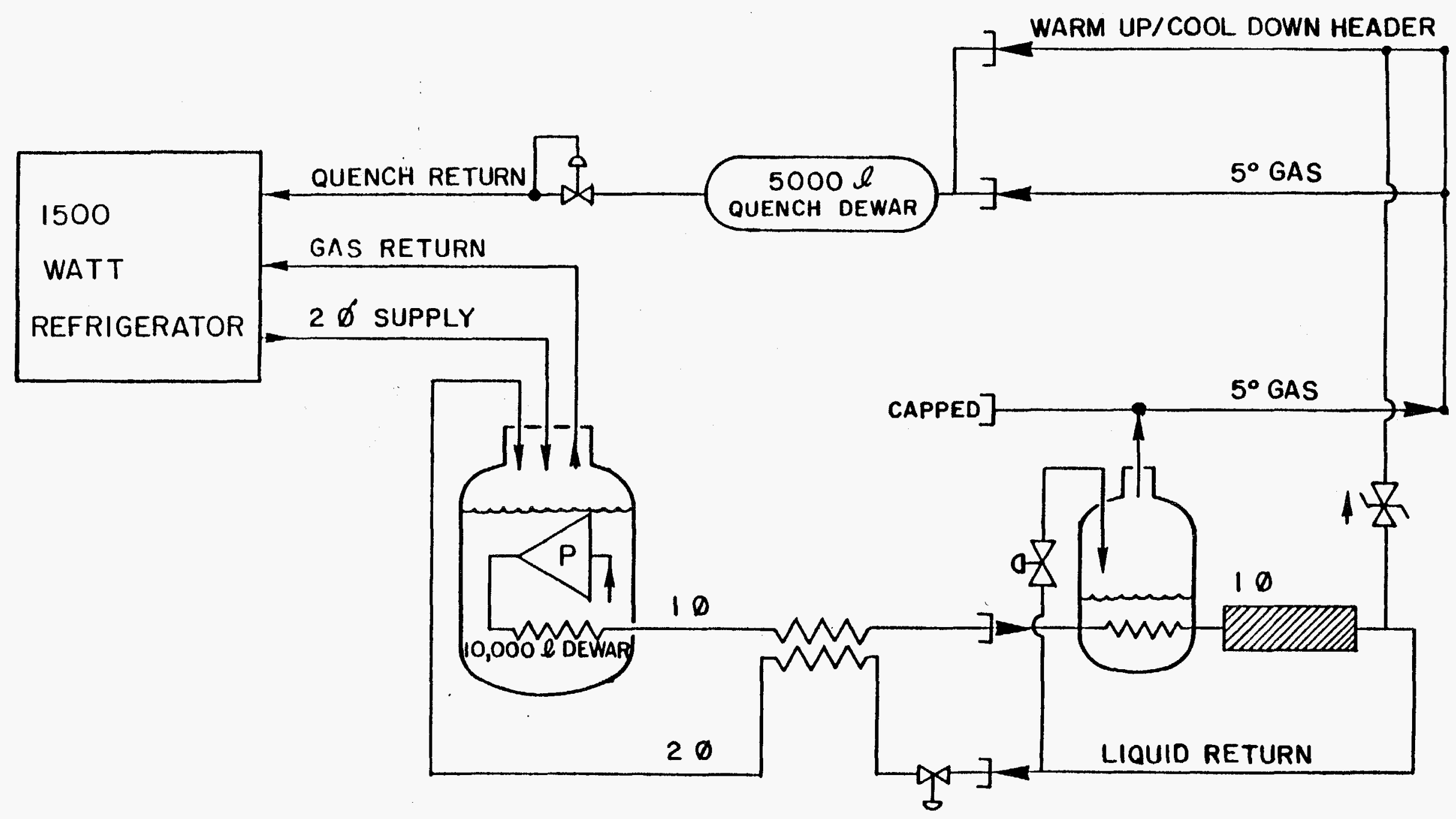

FIG. 4 


\section{MTF 1500 WATT CAPACITIES AND LOADS}

MODES

"Refrigerator"

"Liquefier"

$20^{\circ} \mathrm{K}$

$80^{\circ} \mathrm{K}$
CAPACITY

$1950 \mathrm{~W}$

$13 \mathrm{~g} / \mathrm{sec}$

$20 \mathrm{~g} / \mathrm{sec}$

$50 \mathrm{~g} / \mathrm{sec}$ use auxiliary $\mathrm{N}_{2}$ heat exchanger

5T Static Load: Min. flow $25 \mathrm{~g} / \mathrm{sec}$ maximum flow $100 \mathrm{~g} / \mathrm{sec}$

$5^{0}$ Load 21. W $+19.4 \mathrm{l} / \mathrm{hr}$

$10^{0}$ Load 25. W

$80^{\circ}$ Load 181. W

5T Quench Load: $\quad 6.2 \mathrm{MJ} / \mathrm{half}$ cell quench

1722 watt for one hour quench recovery

861 watt for two hour quench recovery

ST AC (200 sec cycle) $5^{0}$ ? W W

$$
\begin{array}{ll}
10^{\circ} & ? . W \\
80^{\circ} & ? . W
\end{array}
$$

The short string AC heat loads are not known for any of the three magnet types. Using a $200 \mathrm{sec}$ cycle increases hysteresis losses by one order of magnitude and eddy current losses by two orders of magnitude. In addition, the magnet types that use very high conductivity bore tubes may require special tubes for the short string test. 
E-44

SYSTEM TEST FACILITY TIMETABLE

DEC 85

Complete "Zero Dipole" runs

(All instrumentation cross cal ibrated)

(Basel ine heat leak measured)

(Refrigerator controls stabilized)

Jan 86 Start installation one half cell string

Jan 87 Finish installation ten half cell string both aperatures

\section{SHORT STRING FACILITY TIMETABLE}

Dec 85

Magnet type chosen

ilar 86

Detailed design of speciality components finished

July 36 Magnets available short string; install

Oct 86-Oct 87 Run existing list of tests (Type B \& C)

$O R$

Oct 86-Apr 88 Run existing list of test (Type A) 
Short

\section{CRYOGENIC MANPOWER}

The largest cryogenic manpower components are the efforts to install and commission the refrigerator system and the manpower for the initial operation ( one year). We have chosen the M.T.F. 1500 watt refrigerator which is operational and is currently being setup to test design " $B$ " magnets.

This refrigerator does not run unattended and therefore requires a two man crew around the clock. For this budget we are charging half time to the string test. NOTE: The primary duty of the people was installing magnets and taking magnetic measurements. It is obvious that if the short string test is extended or if M.T.F. is not running for magnet measurements, it would be tied into the Tevatron control system and run unattended.

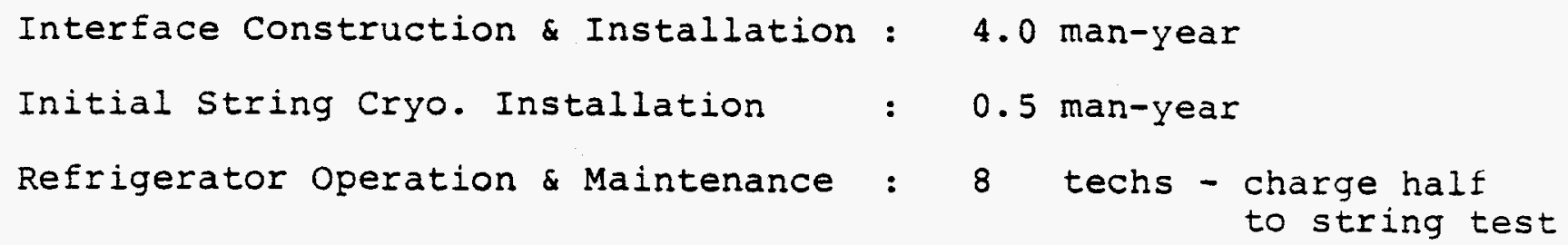

Total manpower: $1 / 1 \quad$ FY85

$1 / 1.5$ FY86

$0 / 5.5$ FY87

$0 / 0 \quad$ FY87 


\section{Short}

\section{OPERATING COST (Design "B")}

POWER

$700 \mathrm{~kW}$ testing program one year half time

$\$ 175 \mathrm{~K}$

LIQ $\mathrm{N}_{2}$

Steady state (Buy $8 \% / l$ ) one year half time

$\$ 50 \mathrm{~K}$

For 100 thermal cycles (Buy $8 \% / \ell$ )

$\$ 35 K$

( $\$ 125 \mathrm{~K}$ design $A)$

Liq He Steady State Leakage

$\$ 50 \mathrm{~K}$

\section{MATERIAL COST FOR INITIAL OPERATION}

\section{Initial Cryo Charge}

$5000 \mathrm{H}$ Hgh pressure quench dewar

(use on long string in FY86)

$80 \mathrm{ft}$. Transfer Line

Misc. Nuts and Bolts
$\$ \quad 1 K(F Y 86)$

$\$ 25 \mathrm{~K}$ (FY85)

$\$ 20 \mathrm{~K}($ FY85)

$\$ 55 \mathrm{~K}$ annual

$\begin{array}{llcccc}\text { BUDGET } & & \text { LABOR } & \text { MATERIAL } & \frac{\text { OPERATING }}{\text { FOTAL }} & \text { TOT } \\ \text { FY85 } & \text { \$100K }(1 / 1) & \$ 95 \mathrm{~K} & 0 & \$ 195 \mathrm{~K} \\ \text { FY86 } & \$ 120 \mathrm{~K}(1 / 1.5) & \$ 6 \mathrm{~K} & 0 & \$ 126 \mathrm{~K} \\ \text { FY87 } & \$ 220 \mathrm{~K}(0 / 5.5) & \$ 55 \mathrm{~K} & \$ 310 & \$ 585 \mathrm{~K} \\ \text { FY88 } & \$ 0 \mathrm{~K}(0 / 0) & \$ 0 \mathrm{~K} & 0 & \$ 0 \mathrm{~K} \\ & & & & \$ 906 \mathrm{~K}\end{array}$

Professional: $\quad \$ 60 \mathrm{~K} / \mathrm{man}$ year

Tech : $\quad \$ 40 \mathrm{~K} / \mathrm{man}$ year 


\section{FERMILAB SSC TEST SITE PROPOSAL \\ POWER SUPPLY/QUENCH PROTECTION FOR LONG TEST STRING}

\section{A. Two Phases}

I. Phase 1

1. Use existing designs to get facility operational at less than full capacity using Energy saver spares or replications with minimum modifications.

2. Provide quench protection for 1 cell.

3. Where economical, modify present designs to provide full capacity to reduce Phase 2 effort.

4. Provide voltage monitoring and heater firing unit locally at quad location in initial system.

II. Phase 2

1. Upgrade facility to provide full power capability for the selected magnet type. Some components cannot be designed until the magnet choice is made.

2. Provide quench protection for 10 half-cells in each of two apertures.

3. As appropriate, replace Energy Saver style equipment with equipment designed specifically for the SSC.

B. Power Supply

I. Phase 1

1. Transformer and Rectifier: Use an E4 main ring power supply reconfigured ( $X$ and $Y$ parallel) to yield $500 \mathrm{~V}$ maximum dc output voltage. With the addition of fans to the transformer, the maximum dc bus current would be $4600 \mathrm{~A}$. Significantly higher currents (approx. $7 \mathrm{kA}$ ) can be achieved, but cycle time must be controlled to keep rms current less than $4600 \mathrm{~A}$.

2. Filter: Use a filter identical to the Energy Saver filter. Significant savings can be realized by using the spare choke initially. This would limit the bus current to something less than $5 \mathrm{kA}$. Higher currents can be achieved with degraded filtering by allowing the choke to operate in a partially saturated mode.

II. Phase 2

1. The FNAL SSC design calls for one transfer and one holding supply per sector per ring. For type A or type B magnets, the E4 power supply could continue to act as a transfer supply and a holding supply of $50 \mathrm{~V}$ and $7 \mathrm{kA}$ could be procured. The $\mathrm{E} 4$ power supply bypass SCR s would be upgraded to higher current devices to allow for dc operation at $7 \mathrm{kA}$. 
2. For type $C$ magnets, the requirement for 3 different power supplies of $12 \mathrm{kA}$ at $120 \mathrm{~V}, 6 \mathrm{kA}$ at $60 \mathrm{~V}$, and $2.5 \mathrm{kA}$ at $100 \mathrm{~V}$ cannot be met with the $E 4$ conversion. Adequate supplies will have to be either located on site or procured. These are key components whose specifications must wait until the magnet choice is made, with a significant lead time to delivery.

3. A new choke should be purchased that fulfills the SSC design requirements. The capacitors and other filter components could probably continue in service.

C. Energy Extraction (Dump)

I. Phase 1

1. The dump will topologically resemble the Energy Saver dumps, except no shunt SCR s are required. The dump uses 8 parallel SCR $s$ and a commutating transformer fed from a charged capacitor bank. A dump of this type in the BO low beta system has been shown to be capable of $6500 \mathrm{~A}$.

2. For redundancy, an Energy Saver dc breaker would be used, imposing a $4600 \mathrm{~A}$ de limitation, but allowing ramping to $7 \mathrm{kA}$ providing the rms current is held to $4600 \mathrm{~A}$. The maximum voltage rating is $2 \mathrm{kV}$, limiting the maximum dump resistance to .28 ohm for $I=7 \mathrm{kA}$.

3. Four Energy Saver dump resistors would be used to provide the nominal .25 ohm, $180 \mathrm{MJ}$ dump resistors. Each Energy Saver dump resistor which consists of two .125 ohm elements has been tested to $45 \mathrm{MJ}$ with repeated dumps 4 min apart. These resistors must be purchased since there are not enough spares on hand.

II. Phase 2

1. The series SCR module from Phase 1 is capable of 7 kA with a blocking voltage of $2500 \mathrm{~V}$. No changes would be necessary if Phase 2 is constrained within these limits. A second series module could be added.

2. An additional dc breaker could be installed in parallel with the Phase 1 do breaker upgrading the de current capability to $9 \mathrm{kA}$.

3. No changes in the dump resistor would be required for test string operation.

4. All dump components are likely to be changed during the test string operation to test these components as designed for the SSC.

D. Current Bypass (If external to cryostat)

I. Phase 1: Two energy Saver Quench Bypass Switch assemblies would be connected in parallel across each bypass unit, giving a $7 \mathrm{kA}$ capability for a dump time constant of $30 \mathrm{~s}$. 
II. Phase 2: As part of the SSC development, if an external bypass circuit is required, a single higher current assembly would probably be developed.

E. Quench Propagation (Heater control - not used for type C magnet)

I. Phase 1

1. An Energy Saver type Heater Firing Unit with a slightly modified control card to allow addressing of the four channels as two pairs will be used.

2. At every other quad electronics station, a local circuit will interface the HFU to a serial link that connects to the QPM in the control room area.

II. Phase 2

1. Ten Energy Saver type Heater Firing Units modified as above will be used.

2. As they are developed, heater control circuits designed specifically for the SSC can replace the original units which can be reworked as Energy Saver spares.

F. Quench Detection

I. Phase 1

1. The control room Quench Protection Monitor will be a direct copy of the present Energy Saver QPM with minor hardware and software modifications. It will initially provide all of the quench detection, protection, system control and data archival duties presently provided in the Energy Saver. It will be connected to the Accelerator Control system in the standard manner.

a. The major hardware modification will be the addition of a new serial link to provide "long distance" communication to the voltage monitoring and heater control circuits located at the electronic stations along the string.

b. Software modifications will be made to integrate the above mentioned serial link and to accommodate the configuration of the test string.

2. An Energy Saver type isolated differential input voltage to Frequency Converter chassis will be used along with a new scaler circuit to provide 4 channels for the first cell. Each measurement will be an integration of the upper or lower half-cell voltage over a $60 \mathrm{~Hz}$ line period.

3. The digital values obtained from these measurements will be collected by the control room QPM every $60 \mathrm{~Hz}$ period using the serial link.

4. The local node electronics will include a microprocessor whose initial purpose will be to connect the serial link to the existing HFU $s$ and the new scaler circuit, and to provide a crystal controlled measurement of the local time period used in the voltage measurement. 
II. Phase 2

1. Five Energy Saver type isolated differential input voltage to Frequency Converter chassis will be used along with the new scaler circuit to provide 8 channels at each of the stations.

2. The local microprocessor can be developed to assume part of the quench protection duties initially provided by the control room QPM. One of the first tasks will be to provide local quench detection for the purpose of direct control of the HFU s. (In the initial configuration, QPM failure will result in global heater firing.)

3. Evolution toward the final SSC configuration can proceed with an orderly transfer of function from the control room QPM into the local stations.

G. System Control

I. Phase 1

1. A direct copy of the Energy Saver TECAR microprocessor will be used to provide ramp control of the power supply via existing Accelerator Control System applications pages. If useful, TECAR will be connected to the QPM as in the Energy Saver to coordinate global quench protection actions and provide voltage regulation programs to the power supply.

2. Both TECAR and the QPM will be connected to the Accelerator Control system via the existing hardware and software used in the Energy Saver.

3. Power Supply status control and monitoring will be provided in the same manner as the Energy Saver.

4. Control of the dump and current bypass systems will be provided in the same manner as the Energy Saver

5. To maintain the same system redundancy as the Energy Saver, two uninterruptable power supplies (UPS) will be needed.

II. Phase 2: Modification of the system control will take place in some manner directed by the SSC design effort. No modification should be necessary for the purposes of test string operation. 


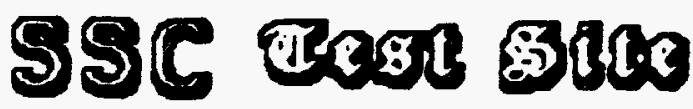

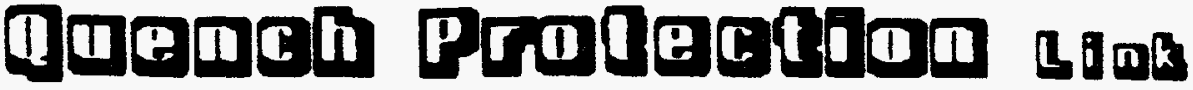

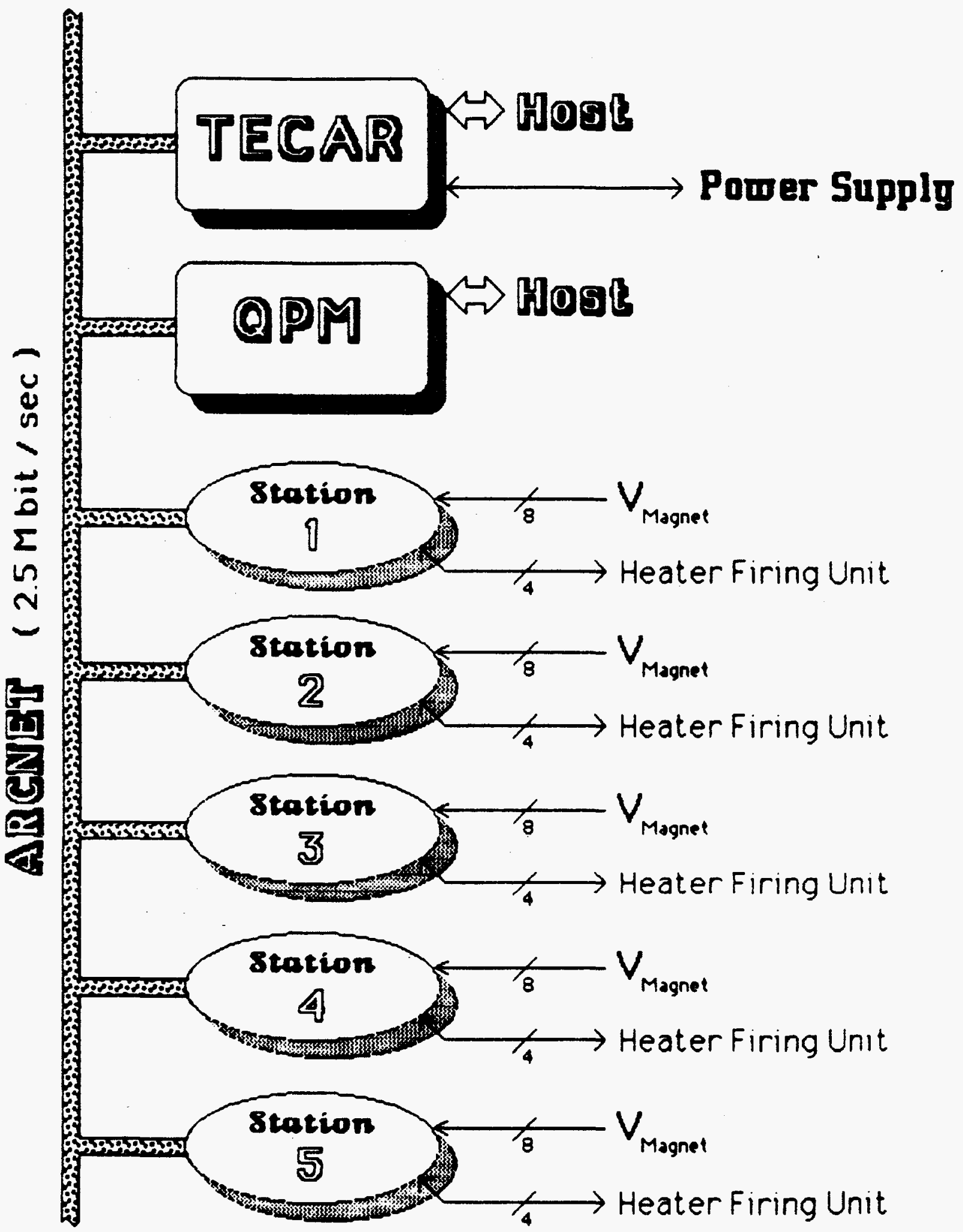




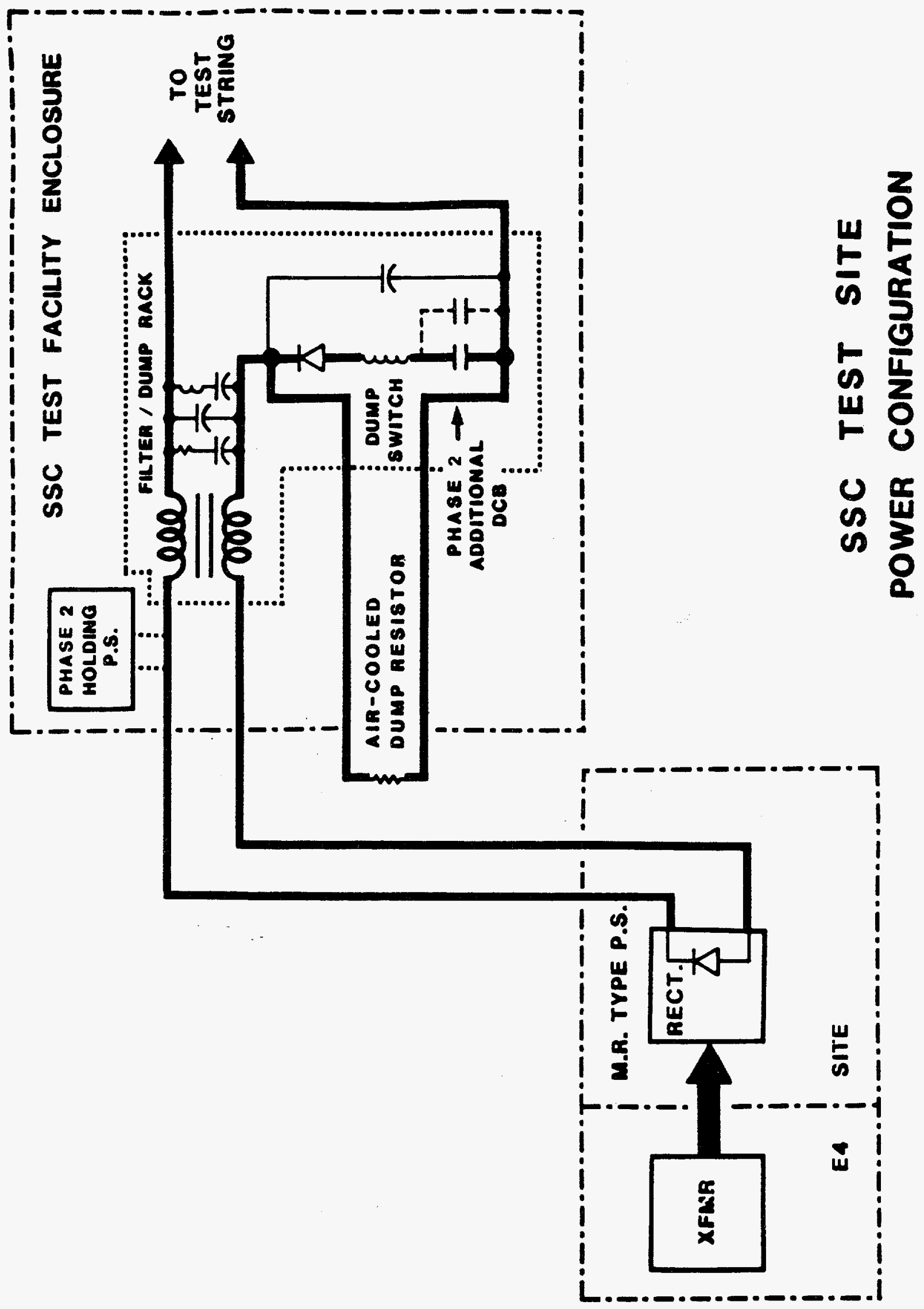


E-53

SSC TEST SITE PROPOSAL

QUENCH PROTECTION and POWER SUPPLY fOr LONG STRING TEST

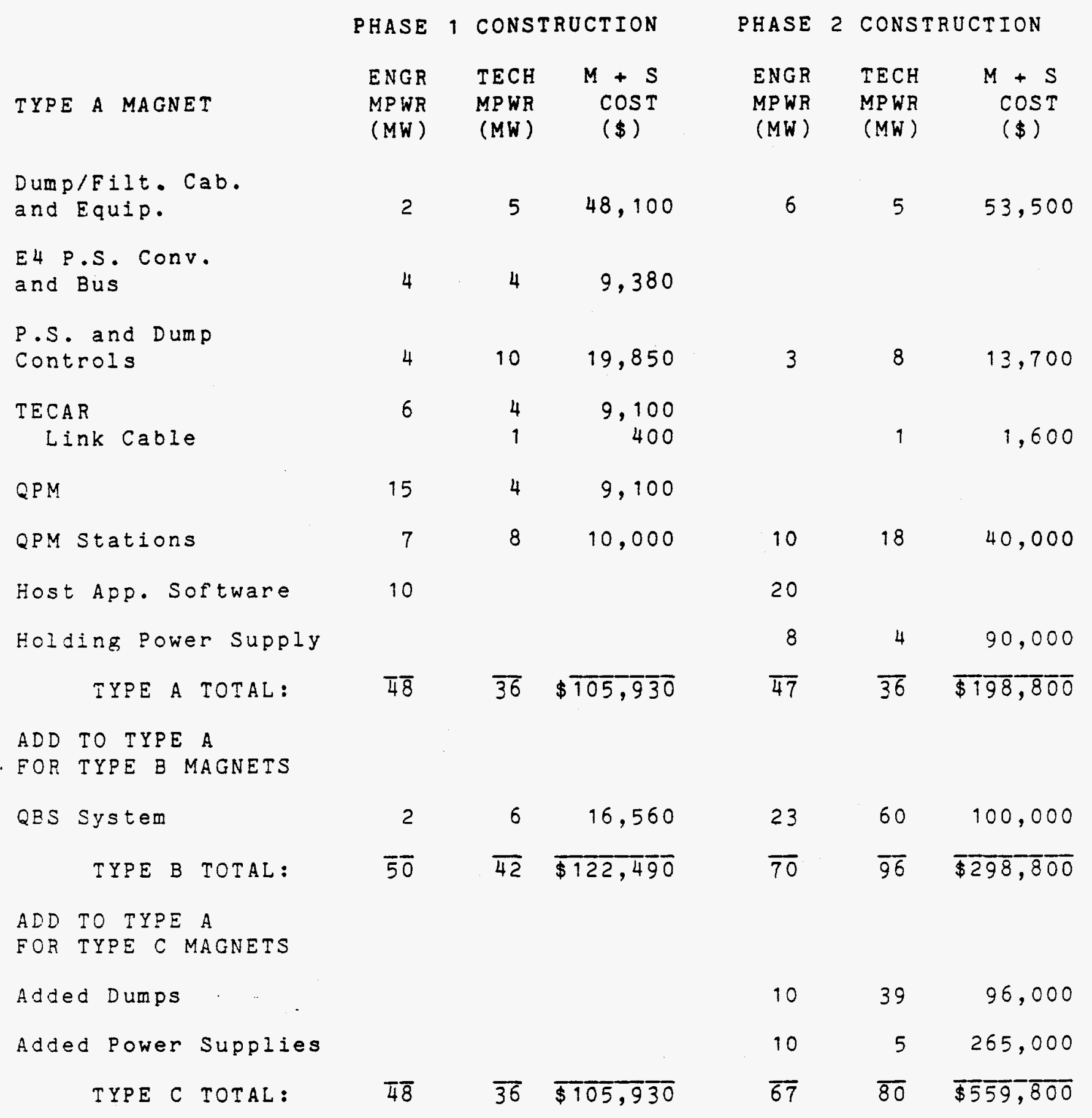

FSSCTSP/90034 
E-54

\section{Controls support for a SSC Long Test String Site}

The assumption of this outline is that the physical location of the long test string site stretches from the approximate location of the E4 refrigerator building southward along Kautz Road to the Butterfield Road gate at the site boundry. a distance of approximately $1.2 \mathrm{~km}$. Furthermore, the assumption is made that the Head House and Control Center for the Long Test String is at the north end of the string: i.e. essentially at the E4 refrigerator/Service Building location.

With these assumptions, and in the spirit of assembling an umbrella control system which would 1) provide the necessary control and data acquisition for the Long String Test Site and 2) provide the possibility of developing into a Host for eventual SSC Local Area Network (Tunnel) instrumentation, the following additions to the existing Fermilab Tevatron system are specified.

1) Connection to the existing Tevatron Serial Link system would be made at E4; a stub line would be run to the Head house/Control center area using RF repeaters. A local console would be installed of the "standard" ACNET variety. This console would require two spare lines in the 19 conductor Heliax and use the "Super-repeaters" currently used to drive the consoles at FO. The distance to E4 is just slightly greater than from $A O$ to $F O$; no problems are anticipated in supporting such a console. One would then automatically have access to all ACNET features: logging, alarms, save-comparerestore, etc. etc., as well as excellent software development facilities for both applications and analysis, as well as for possible subsystem development.

2) One must support the additional refrigerator at E4. This would initially mean another "Frig" multibus unit for the refrigeratori more if for any reason there were more control or monitoring points required.

3) For any test purposes requiring Vacuum readback and control where necessary (transfer lines?, verify good magnet vacuum? the existing Vacuum 170 and CIA crate modules should be a good start.

4) For excitation of correction coil elements the existing ramp generators should be adequate. (160's, 165's, HOGS)

5) With the possible exception of the QPM's, application code for existing services should be adequate for rapid turn on. Standard MADC services with 190 modules may also be used to support slow or fast time plots. Again. with the possible exeeption of the QPM microcomputer, no substantial microcomputer coding should be necessary either.

6) To be sure that there will be unambigous storage available for logging, one would add two more RAbO's to the OPER VAX; although in all likelihood, there is more than enough storage now available.

Note: While both dipole excitation and quench protection might be considered as part of the Controls system, these systems are addressed in the separate section on Power Supplies and Quench Protection and so are neither deseribed or costed here. 
1) Links, Crates, Consoles, ete.
a) Heliax 200'7 lines
b) Install Heliax, terminate
$\$ 2 K$
c) RF repeaters ( 10 modules)
$\$ 3 K$
d) Repeater crates and supplies
$\$ 1 K$
e) 2 CAMAC Crates with supplies
$\$ 3 K$
f) 2 Crate controllers
$\$ 3 K$
g) 5 Relay racks
$\$ 2 K$
h) Complete Console (Value - \$100K)
$\$ 3 K$
Subtotal
exists
$\$ 17 K$

2) Refrigerator Multibus system

$\begin{array}{ll}\text { a) Multibus erate and supply } & \$ 2 K \\ \text { b) Micro-computer } & \$ 1 K \\ \text { c) MADC card } & \$ 2 K \\ \text { d) G other cards (MOBO, MO11, M012 etc) } & \$ 3 K \\ \text { e) Connectors (But no cables) } & \$ 1 K\end{array}$

Subtotal

$\$ 9 K$

3) Vacuum 170, CIA Crate etc.

a) Vacuum 170 module

b) CIA crate and supply

$\$ 1 K$

$\$ 2 K$

c) Sundry cards

$\$ 4 K$

Subtotal

$\$ 7 K$

(Note: no vacuum pumps, ion gauges etc. in this)

4) Let's say half dozen CAMAC 160 ramp generators

Subtotal \$6K

5) MADC crate and 190 Module

Subtotal \$8K

6) Add 2 RAGO Disk drives to OPER VAX

Subtotal

$\$ 35 K$

Grand Total

$* * * * * * * *$

$\$ 82 K$

$* * * * * * * *$

The original estimate included in the Fermilab oraft for

manpower for design, fabricate, and install those parts

outlined above remains: (In MY of effort)(Staff/Weekly)

Design/Fab Install

Sum
Controls Hardware
0. $5 / 1.0$
0. $0 / 0.5$
$0.5 / 1.5$
Controls Software
1. $0 / 0.0$
0. $0 / 0.0$
1. $0 / 0.0$
Total
which is
1. $5 / 1.5$
$\$ 150 K$ 
Continuing Operation Manpower:

In a steady state operation, discounting the actual evolution towards an SSC control system. it is our estimate that essentialiy no hardware effort would be charged, but that up to two fulltime software equivalents would be required to help alter/improve/adjust operation and data taking to the specifications of the test coordinators. This cost is felt. however, to properly belong to the SSC $R$ and $D$ budget and so is not included here.

Evaluation of controls effort:

This type of support could be provided on a very short time scale at Fermilab, with the possible exception of the QPM microcomputer code. (That means: AD/Controls could have the rest ready to go in one week starting today if the remote controls enclosure existed and we stole a console and all other components from existing spares.) The addition of a new console is possible at this time, but would take several months to order and assemblei similarly, the replacement of other components would require several ( 4 to 6?) months to accomplish.

Depending on the differences and complexity

of the QPM work, anywhere from a few months to quite a bit of programming effort could be required for the QPM micro computer and possibly the teststring TECAR. 
Controls support for a SSC short test string

The assumption of this outline is that the physical location of the short test string site stretches out the west side of the MTF area (Industrial Bldg \#1) and that the existing MTF test facilities are directly connected.

With these assumptions, the four tests outlined in the test specifications (and possibly some magnetic measurements as well?) could be supported with the existing MTF measuring system. which includes scheduled upgrades replacing the present PDP-11/34 with the VAX 11/730 being used for TeV I magnet measurements at present. The specified tests do not call for any substantial alterations of existing single magnet test set ups or data acquisition methods. It is possible, however, that by Short string test set up time the MTF system may be networked into Fermilab networks, making file transfers to other locations (Cluster VAXes. AD/DEVL cluster, etc.) quite routine. Therefore, no substantial alterations or additions to existing MTF facilities are required, or costed in this document for controls or data acquisition purposes.

It would be imprudent, however, not to at least included some contingent software effort, perhaps 1 MY during installation and $1 / 2$ full time equivalent (or less? during operation.

Installation

Qperation
$\$ 60 K$

$\$ 30 K /$ year 
5. SAFETY

We are assuming a $3500^{\prime}$ building with 21 entrances/exits at 60 meter intervals. Entrances are allowed only at doors $1,7,14$ and 21 , which are complete with full interlocks. other doors are only emergency exits but are interlocks.

Entrance points have controlled access, $0_{2}$ monitors, and an exhaust fan controlled from 02 monitors.

Fully interlocked entrance points (four):

$\mathrm{O}_{2}$ monitors $\$ 800$

Fans $\$ 200 \times 4=\$ 5,400$ material

Interlocks $\$ 350$

$\$ 1,600$ labor

Interlocked doors only (17):

\begin{tabular}{|c|c|c|c|}
\hline$\$ 50 \times 17$ & $=$ & $\begin{array}{l}\$ 850 \\
\$ 1,000\end{array}$ & $\begin{array}{l}\text { material } \\
\text { labor }\end{array}$ \\
\hline Junction boxes, power & & & \\
\hline supplies, master modules & $=$ & $\begin{array}{l}\$ 2,000 \\
\$ 1,000\end{array}$ & $\begin{array}{l}\text { material } \\
\text { labor }\end{array}$ \\
\hline Cables & $=$ & $\begin{array}{l}\$ 4,000 \\
\$ 1,000\end{array}$ & $\begin{array}{l}\text { material } \\
\text { labor }\end{array}$ \\
\hline
\end{tabular}

TOTAL $\$ 18,850$ 